
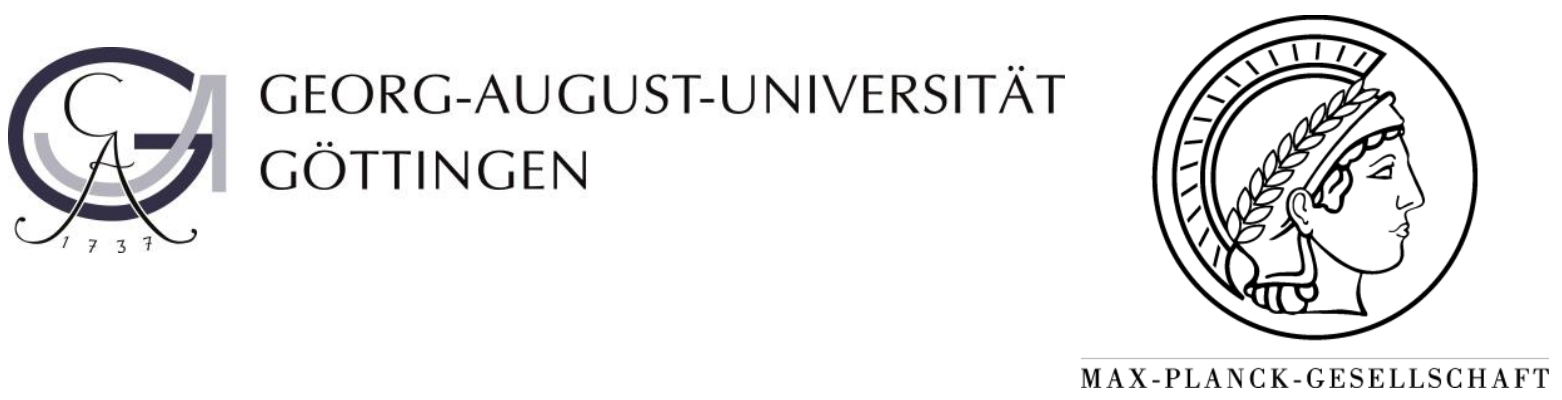

\title{
Smu1 and RED play an important role for the activation of human spliceosomes
}

\section{Dissertation}

\author{
for the award of the degree \\ „Doctor rerum naturalium“ \\ of the Georg-August-Universität Göttingen \\ within the doctoral program "Molecular Biology of Cells" \\ of the Georg-August University School of Science (GAUSS)
}

\author{
submitted by \\ Sandra Maria Keiper \\ from Berlin
}

Göttingen, 2018 


\section{Members of the Examination Board:}

Prof. Dr. Reinhard Lührmann (1 ${ }^{\text {st }}$ Referee)

Department of Cellular Biochemistry

Max Planck Institute for Biophysical Chemistry, Göttingen

Prof. Dr. Heike Krebber (2nd Referee)

Department of Molecular Genetics

Georg-August-Universität Göttingen

Prof. Dr. Patrick Cramer

Department of Molecular Biology

Max Planck Institute for Biophysical Chemistry, Göttingen

Prof. Dr. Markus Zweckstetter

Department for NMR-based Structural Biology

Max Planck Institute for Biophysical Chemistry, Göttingen

Prof. Dr. Jörg Großhans

Department of Developmental Biochemistry

University Medical Center Göttingen

Prof. Dr. Ralph Kehlenbach

Department of Molecular Biology

University Medical Center Göttingen 
For Oskar 



\section{Table of Contents}

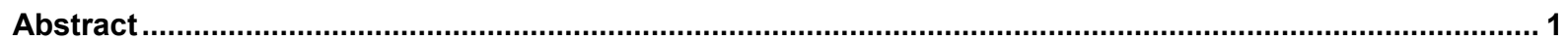

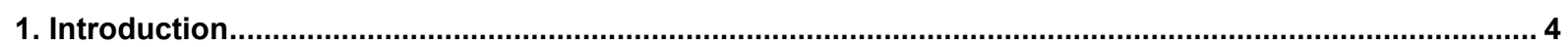

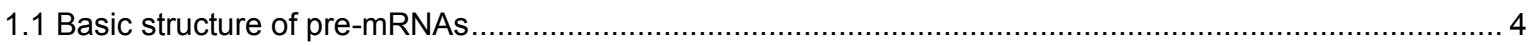

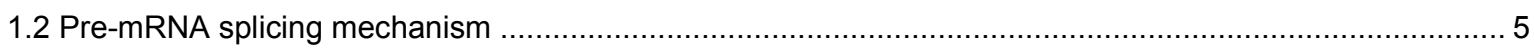

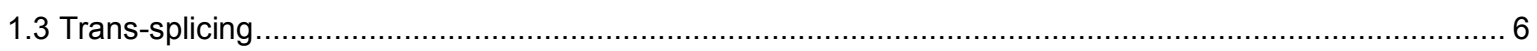

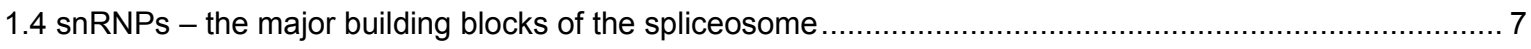

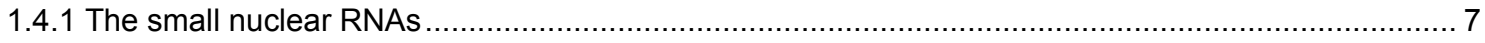

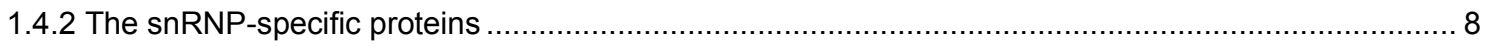

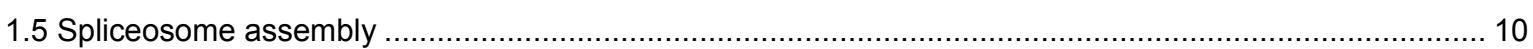

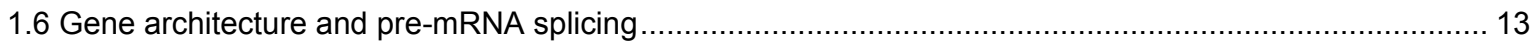

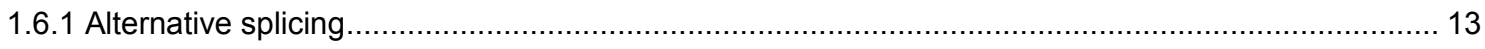

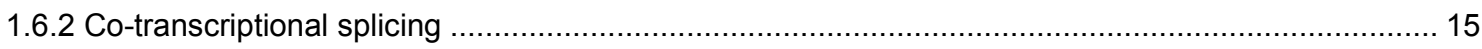

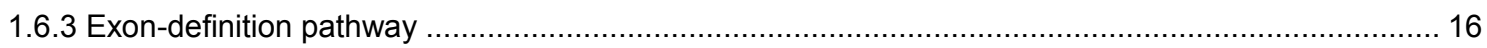

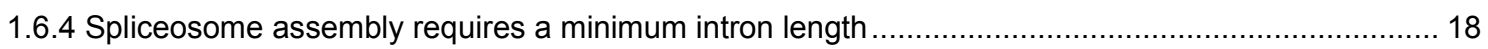

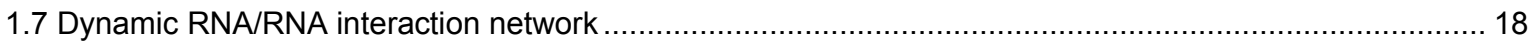

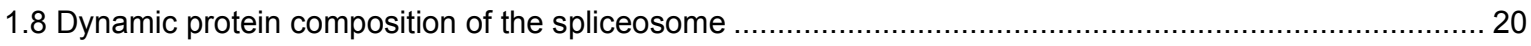

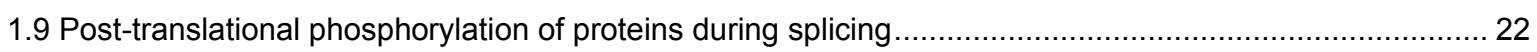

1.10 The structure of the human B complex and the organization of the B-specific proteins .......................23

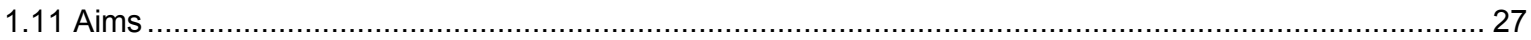

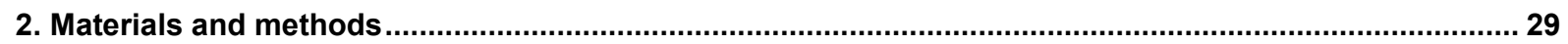

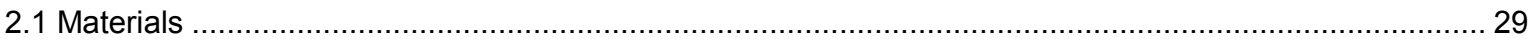

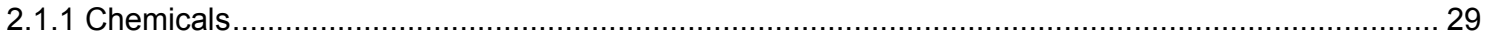

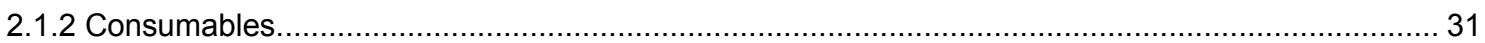

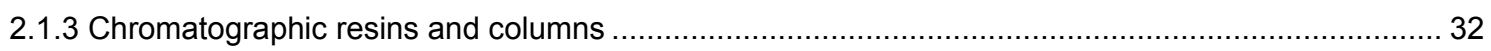

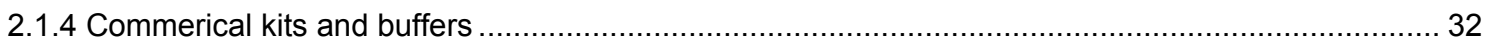

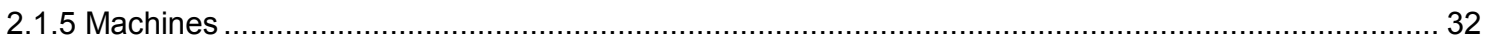

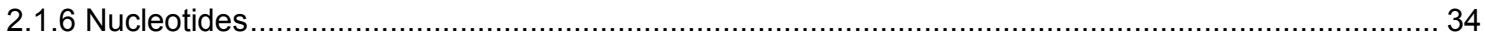

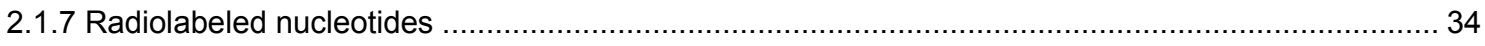

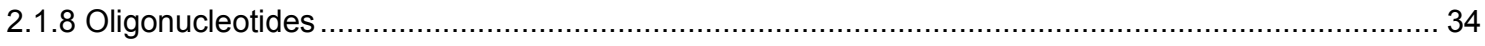

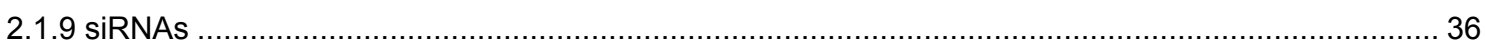




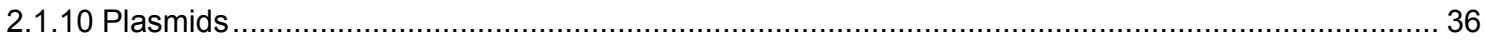

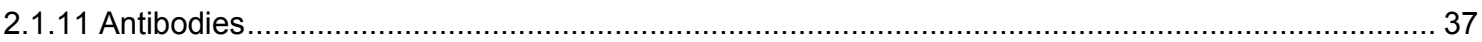

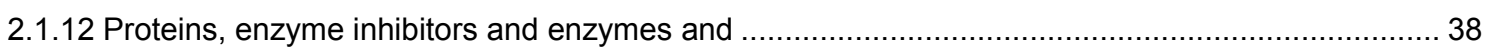

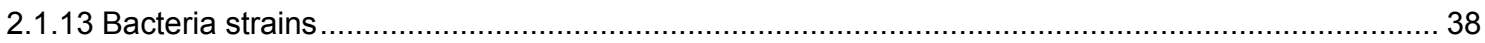

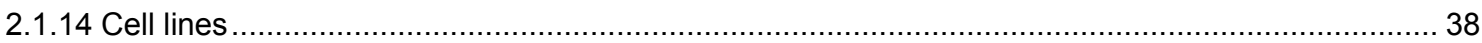

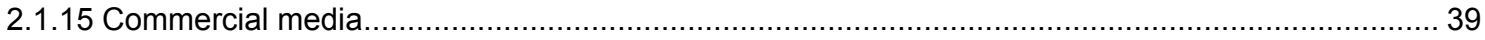

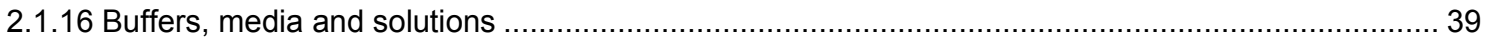

2.2 Methods

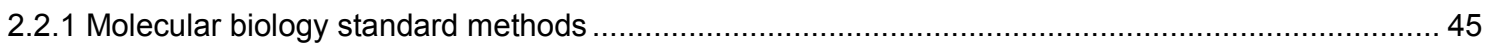

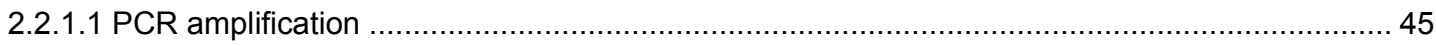

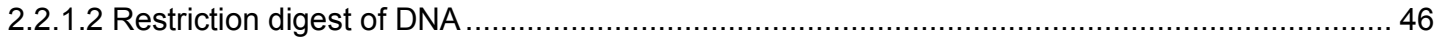

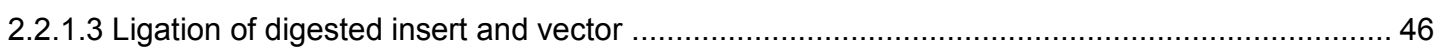

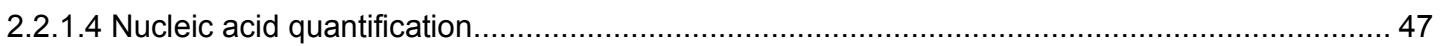

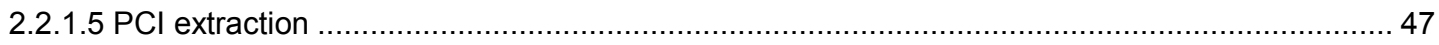

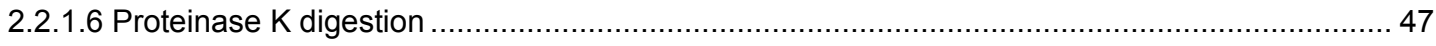

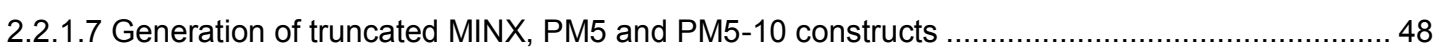

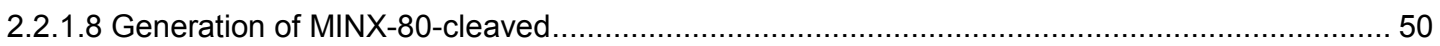

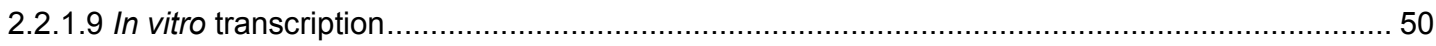

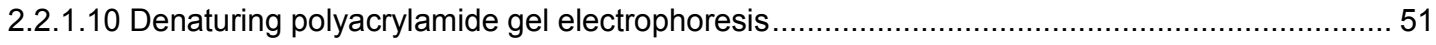

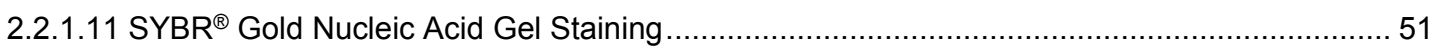

2.2.1.12 Agarose gel electrophoresis of nucleic acids .......................................................... 51

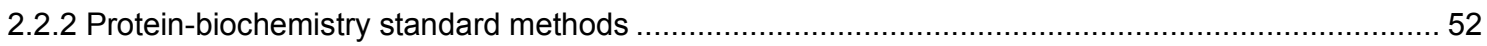

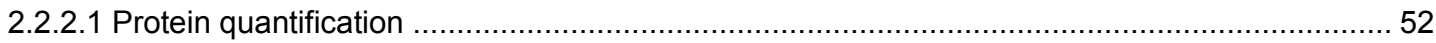

2.2.2.2 Denaturing SDS polyacrylamide gel-electrophoresis (SDS-PAGE) …........................... 52

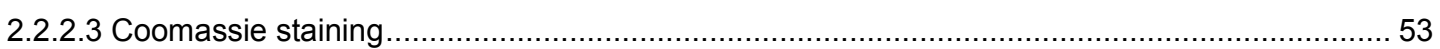

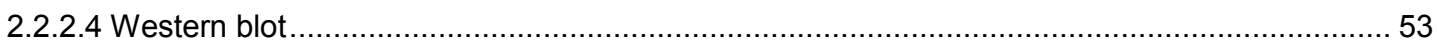

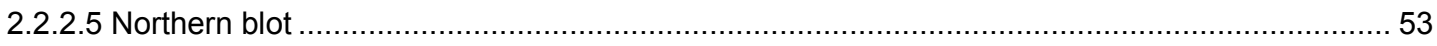

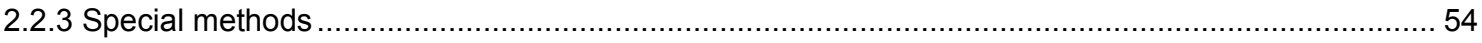

2.2.3.1 Preparation of splicing active HeLa nuclear extract ..................................................... 54

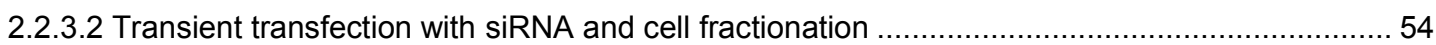

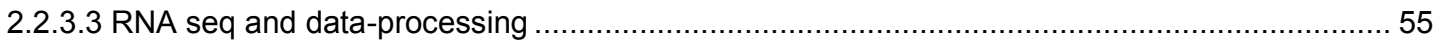

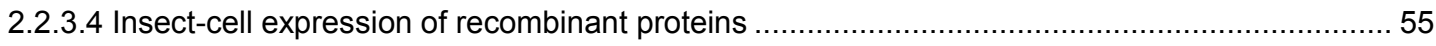

2.2.3.5 Purification of recombinant proteins from insect cells .................................................... 56

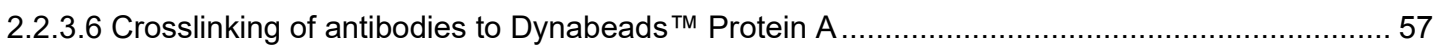

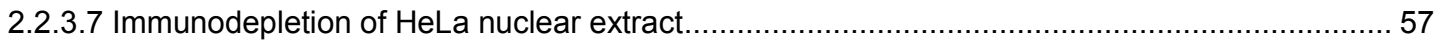

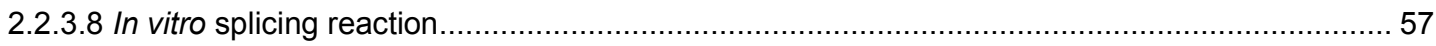

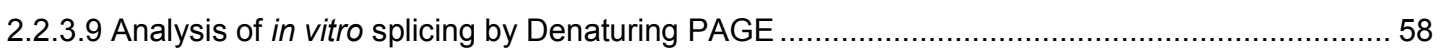

2.2.3.10 Analysis of splicing complexes by native agarose gel-electrophoresis .............................. 58

2.2.3.11 MS2 affinity-selection of splicing complexes ......................................................... 59 
3.1 Alternative and constitutive splicing are affected by knockdown of Smu1 and RED

3.8 Smu1/RED immunodepletion does not affect the recruitment of additional spliceosomal factors to the B complex

3.9 The distance between the 5'SS and the branch site, rather than total intron length per se, is the decisive factor for Smu1/RED dependence

3.10 Cleaving the MINX-80 substrate into two parts restores activation on short introns independently of Smu1/RED

3.11 Stalled $\Delta$ Smu1/RED B complexes can be activated by addition of the purified dimer

3.12 The interaction between Smu1 and RED is essential for their function in splicing

3.12.1 Smu1 and RED can only support the activation co-operatively

3.12.2 The WD40 domain of Smu1 is required for association of Smu1/RED with the spliceosome ..........85

3.12.3 Structural requirements of RED for supporting $B^{\text {act }}$ complex formation

3.13 MINX-70 stalls spliceosome assembly after tri-snRNP association but before efficient U1 displacement 88 3.14 Smu1 and RED are required for spliceosome activation in vivo. 93

4. Discussion

4.1 Efficient splicing is dependent on Smu1 and RED in vivo and in vitro .... 
4.7 Smu 1 and RED might directly affect Brr2 helicase activity during the earliest step of activation

4.9 The distance between the 5'SS and the BS is critical for splicing and determines whether Smu1 and RED are essential.

4.10 A short 5'SS-BS distance represents a steric hindrance for the spliceosome. 109

4.11 Model for how the 5'SS-BS distance affects the dependency of spliceosome activation on Smu1/RED 109

4.12 The tri-snRNP still binds the MINX-70 pre-mRNA.

4.13 Perspectives

6. Appendix .

6.1 Abbreviations

6.2 List of figures.

6.3 List of tables

6.4 Acknowledgements 


\section{Abstract}

In eukaryotes, pre-mRNA splicing is catalysed by the spliceosome, a highly complex and dynamic molecular machine, which assembles stepwise by the sequential recruitment of five small nuclear ribonucleoproteins (snRNPs) and numerous non-snRNP proteins. In humans, formation of the pre-catalytic, spliceosomal B complex with a stably associated tri-snRNP requires the action of the helicase Prp28, which displaces the U1 snRNP from the $5^{\prime}$ splice site to be replaced by the U6 snRNA. At the same time, the so-called, B-specific proteins are recruited. Transformation of the $B$ complex into an activated $\left(B^{\text {act }}\right)$ spliceosome is initially triggered by the action of the helicase Brr2 that unwinds the U4/U6 RNA duplex. This results in the crucial release of the U4 snRNP, which allows the U6 snRNA to interact with the U2 snRNA forming essential components of the catalytic centre. Concomitantly, the B-

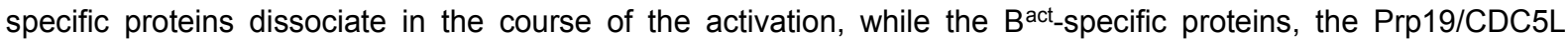
complex and the intron binding complex (IBC) are stably integrated.

Smu1 and RED are two B-specific proteins that are conserved among higher eukaryotes but absent in S. cerevisiae. Currently, little is known about the precise function of these proteins. Their transient association with the B complex suggests that they may be involved in the B-to-Bact complex transition, but previous studies proposed that these proteins form a functional module that is involved in the regulation of alternative splicing. However, it is not clear how Smu1 and RED might contribute to this process. Furthermore, it has not been investigated whether these proteins participate in constitutive splicing and thus it remains possible that Smu1 and RED - like several other Bspecific proteins - are in general important for intron excision in higher eukaryotes. To provide clarity on this subject, I analysed the function of Smu1 and RED in pre-mRNA splicing both in vivo and in vitro.

To address whether constitutive splicing is dependent on Smu1 and RED, an RNAseq analysis was performed with HeLa cells siRNA-depleted of Smu1 or RED. Knock-down of these two proteins resulted in profound changes in alternative splicing patterns and also led to the retention of constitutively spliced introns, suggesting that Smu1 and RED are important for splicing in general, and thus not only involved in the regulation of alternative splicing. A role for Smu1 and RED in constitutive splicing was also demonstrated in vitro, using HeLa nuclear extract that was immunodepleted of Smu1 and RED. By using a well-functioning Smu1-specific antibody, Smu1 was nearly quantitatively removed from the extract along with more than $90 \%$ of RED, suggesting that the majority of human Smu1 and RED exist as a dimer in HeLa cell extract. Splicing of MINX-120 was less efficient in the absence of Smu1/RED, with an apparent slow-down in the rate of mRNA production. Investigation of spliceosome assembly revealed a transient accumulation of spliceosomal B complexes in the Smu1/RED-depleted extract, while the subsequently formed $\mathrm{B}^{\text {act }}$ and $\mathrm{C}$ complexes were still formed but at a slower rate. These results indicate that defects in splicing triggered by the absence of Smu1 and RED were caused by impaired spliceosome activation.

To investigate whether Smu1 and RED need to interact to fulfil their function, spliceosome assembly in the presence of the individually expressed proteins was investigated. As the addition of single proteins to the Smu1/RED-depleted extract did not restore the B-to-B ${ }^{\text {act }}$ transition and splicing product formation, Smu1 and RED do not appear to function on their own. This was attributable to poor or less stable binding to the spliceosome of the individual 


\section{Abstract}

proteins compared to the Smu1/RED dimer. Removal of the WD40 domain of Smu1 abolished binding of the dimer to the spliceosome, and spliceosome assembly remained blocked at B complex level, demonstrating that the WD40 domain is essential for proper interaction of the dimer with the spliceosome. Truncation of RED's N-and C-terminal regions, which contact U2 or tri-snRNP proteins, respectively, within the B complex, restored splicing and spliceosome activation partially or nearly fully, suggesting that the contacts RED establishes with U2 or U5 individually are not essential for the function of Smu1/RED. To determine whether Smu1 and RED function as a binding platform for other spliceosomal factors, I purified B complexes that accumulate in their absence and investigated their composition. As no additional proteins were missing, Smu1 and RED appear to play a direct role in splicing, as opposed to aiding the binding of other factors required for spliceosome activation. Thus, these results indicate that Smu1 and RED themselves are important for efficient conversion of the B complex into $\mathrm{B}^{\text {act }}$.

To provide evidence that Smu1 and RED are also involved in spliceosome activation in vivo, I knocked-down these proteins in HeLa cells and investigated endogenous spliceosome assembly by immunoblotting, using antibodies that recognise phosphopeptides specifically associated with assembled B or B ${ }^{\text {act }}$ complexes. Knock-down of Smu1 or RED led to an increased B complex signal and a decreased $\mathrm{B}^{\text {act }}$ complex signal, indicating that the activation of the spliceosome is also impaired in vivo in the absence of Smu1 and RED.

While knock-down of Smu1 and RED affected introns of all sizes, the vast majority were shorter than 100 nt. Short introns constitute only a small fraction of introns in the human genome, and thus this result indicates that the splicing of very short introns in vivo is highly dependent on the presence of Smu1 and RED. In vitro splicing studies using truncated versions of the MINX-120 pre-mRNA and Smu1/RED-depleted extract, also demonstrated that splicing was more dependent on the presence of Smu1 and RED when intron length was shorter. Truncation of the intron to either 90 or $80 \mathrm{nt}$ reduced the overall efficiency of splicing compared to MINX-120, but it also enhanced the inhibitory effect of Smu1/RED-depletion. While MINX-90 was spliced somewhat less efficiently than MINX-120, splicing of MINX-80 was nearly abolished in the absence of Smu1 and RED, and led to an apparent block at the B complex stage with little or no formation of $B^{\text {act }}$ or catalytically-active C complexes. Thus, Smu1 and RED play a crucial role in the splicing of extremely short introns both in vitro and in vivo. To investigate whether the intron length per se or the distance between the 5'SS and the BS or between the BS and the 3'SS determines whether splicing is dependent on Smu1/RED or not, I compared spliceosome assembly on PM5 pre-mRNAs with a shortened 5'SSBS distance or a shortened polypyrimidine (PY) tract (BS-3'SS distance).

While spliceosome activation was not affected by the truncation of the PY tract in the absence of Smu1 and RED, shortening of the 5'SS-BS distance to $\sim 55 \mathrm{nt}$ (as found in MINX-80) blocked the assembly at the B complex level, indicating that the distance between the 5'SS and the BS is the decisive factor for a strong Smu1/RED-dependence.

The dependence of spliceosome activation on a minimal 5'SS-BS distance is probably due to physical limitations exerted by the intron. In this case, resolving this steric hindrance should allow spliceosome activation even without the support of Smu1 and RED. Indeed, when the MINX-80 pre-mRNA was cleaved into two RNAs, spliceosome activation was restored in the absence of Smu1/RED. This supports the idea that a short 5'SS-BS distance exerts a structural constraint, which can be relieved by physically separating the $5^{\prime}$ and 3 ' regions of an intron. 


\begin{abstract}
Based on my data and structural information obtained from the cryo-EM structure of the human B complex, a model for how 5'SS-BS distance might lead to the dependency of spliceosome activation on Smu1/RED was generated. In the human B complex, Smu1 and RED form a molecular bridge between the U2 snRNP protein SF3B3 and the RNA helicase Brr2. This interaction appears to be important for spliceosome activation, potentially either by tethering Brr2 in a position required to unwind the U4/U6 interaction during activation or by directly aiding in triggering Brr2 unwinding activity. In the B complex, the intron is base paired with the U6 ACAGAG box at the 5'SS and with the U2 SnRNA at the BS region. In most cases the distance between the 5'SS and the BS of the intron is sufficiently long to grant the U2 domain enough flexibility to move towards Brr2 and to form a bridge directly, even when Smu1 and RED are absent, albeit it at a slower rate. However, when this distance is short, i.e. $\sim 56$ nt or less, the intron adopts a fully extended conformation that results in a structural constraint. This could potentially result in repositioning of the U2 domain away from Brr2 and/or hinder its ability to move towards Brr2, and in turn inhibit spliceosome activation. As Smu1 and RED extend the U2/Brr2 bridge, the negative effect of a short 5'SS to BS distance would be greatly enhanced in their absence. Taken together, my studies provide novel insights into the function of the B-specific proteins Smu1 and RED in splicing. They additionally elucidate how intron architecture impacts spliceosome assembly and splicing, and how spliceosomal proteins potentially help the splicing machinery to overcome the challenges created by short introns.
\end{abstract}




\section{Introduction}

\section{Introduction}

It has been more than 70 years since deoxyribonucleic acid (DNA), was identified as the molecule in which the genetic information of an organism is stored (Avery et al., 1944; Watson \& Crick, 1953). The conversion of this information into a functional protein is a laborious process and increases in complexity with increasing complexity of the organism. In simple prokaryotic organisms, a gene consists of a continuous DNA sequence that is transcribed in its entirety and is directly translated into a protein (Jacob \& Monod, 1961). In more complex eukaryotic organisms, a gene that is transcribed in the nucleus by the RNA polymerase II needs to undergo several processing/maturation events before it can be exported to the cytoplasm, where it is translated into a protein by the ribosomal machinery (Clawson et al., 1985).

The processing of the primary transcript, also called precursor messenger RNA (pre-mRNA), already begins during its transcription. Right after the appearance of its 5' end, a modified nucleotide, called N7-methylguanosine $\left(\mathrm{m}^{7} \mathrm{G}\right)$, is attached to the $5^{\prime}$ ribose by a rare 5'-to-5' triphosphate linkage (Shatkin, 1976; Chiu et al., 2002). Towards the end of transcription, but before termination, the transcript is cleaved at the 3 ' end, and this is followed by the synthesis of a poly $(\mathrm{A})$ tail comprising up to 200 adenosine residues. Both modifications are important for the transcript's stability and further promote its export to the cytoplasm and its productive translation (Shatkin \& Manley, 2000; Rosonina et al., 2003; Nagaike et al., 2011).

In eukaryotic genes, especially of higher eukaryotes, protein-coding sequences termed exons are often disrupted by non-coding sequences termed introns, which are still included in the primary transcript. This demands an additional maturation step called splicing; in this step, intronic sequences are excised and the remaining exons are ligated together (Berk, 2016). The spliceosome, a highly complex and dynamic molecular machine, catalyses the splicing reaction and ensures its accuracy.

\subsection{Basic structure of pre-mRNAs}

The first demanding task of the spliceosome is the definition of the intron-exon boundaries. Since defects in splicing can have dramatic consequences for the cell and can ultimately cause severe diseases such as cancer or neurodegenerative diseases in humans, the exact nucleotide identification of an intron's ends is a crucial process (Cooper et al., 2009; Webb et al., 2013; Scotti \& Swanson, 2016). In eukaryotes, including yeast and humans, introns are flanked by short conserved sequences called splice sites, which define the boundaries of the intron and are important motifs for intron/exon recognition by the spliceosome (Fig. 1.1).

The 5' splice site (5'SS) sequence marks the boundary between an intron and its upstream exon. While this sequence (AG|GUAUGU, where 'l' indicates the exon/intron boundary) is highly conserved in yeast (Lopez \& Séraphin, 1999), it is much more variable in human cells (AG|GURAGU, where R = purine [G or A]) (M. Q. Zhang, 1998). The boundary between an intron and its downstream exon is determined by the 3' splice site (3'SS). In yeast and human, this consists of the characteristic sequence YAG (Y = pyrimidine [C or U]) (M. Q. Zhang, 1998; Lopez \& Séraphin, 1999). Another essential element is the branch-point sequence (BPS) YURAY (human) (M. Q. Zhang, 
1998) or UACUA드 (yeast) (Lopez \& Séraphin, 1999; Spingola et al., 1999). This motif is localized 18-40 nt or 10$40 \mathrm{nt}$ respectively, upstream of the 3'SS. Most human introns further contain a polypyrimidine (PY) tract. This pyrimidine-rich region is located between the branch site (BS) and the 3'SS and helps to recruit and stabilize the interaction of spliceosomal factors with the pre-mRNA and is an important splicing motif in humans (Coolidge et al., 1997).

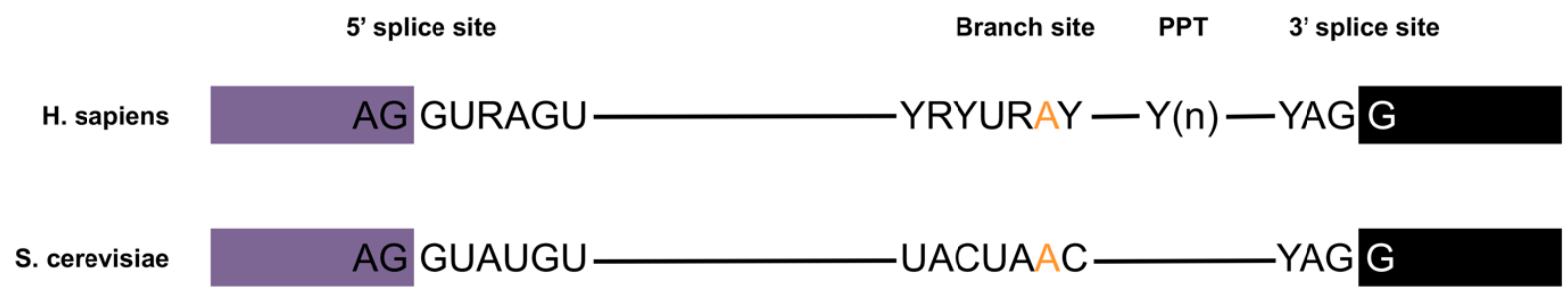

Figure 1.1. Conserved splicing sequences of pre-mRNA from $\boldsymbol{H}$. sapiens and S. cerevisiae. Schematic representation of a pre-mRNA comprising one intron (black line) surrounded by two exons (purple and black boxes). The conserved intron defining consensus sequences are specified in black and white letters, while the branch-point adenosine is shown in orange. $\mathrm{Y}(\mathrm{n})$ indicates the polypyrimidine tract. $(\mathrm{Y}$ : pyrimidines (C or T), R: purines (A or G). (Will \& Lührmann, 2011)

\subsection{Pre-mRNA splicing mechanism}

The excision of introns from a pre-mRNA includes two sequential $S_{N} 2$-type transesterification reactions (Fig. 1.2). During the first reaction, the 2' hydroxyl group of the branch-point adenosine attacks nucleophilically the phosphodiester bond of the 5'SS. This results in the liberation of the 5' exon and formation of the 3' exon-intron lariat intermediate, in which the branch-point adenosine and the guanosine at the intron's 5' end are connected by a 2'-5' phosphodiester bond. The second splicing reaction involves the free 3' hydroxyl group of the 5' exon, which performs a nucleophilic attack on the phosphodiester bond at the 3'SS, resulting in exon ligation and the release of the intron lariat (Moore \& Sharp, 1993).

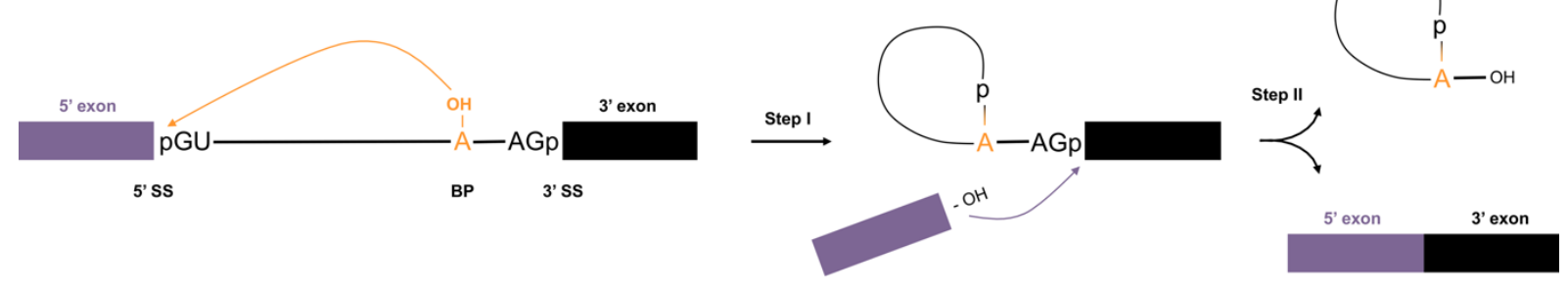

Figure 1.2. Chemistry of the splicing reaction. Schematic illustration of the two sequential transesterification reactions. The first step comprises nucleophilic attack by the 2 ' $\mathrm{OH}$ group of the branch-point adenosine on the phoshodiester bond at the 5' splice site, leading to the free 5' exon and intron-3' exon intermediates. During the second step the free 3' OH group of the 5' exon targets the phosphodiester bond at the 3'SS, resulting in the ligation of the two exons and the release of the intron lariat. Phosphate groups involved in the reactions are indicated by "p". (Will \& Lührmann, 2011) 


\section{Introduction}

The mechanism of pre-mRNA splicing resembles that of group II self-splicing introns. Both exhibit similar consensus sequences at the splice sites and branch point and form identical intermediate and end products in two transesterification reactions. Since pre-mRNA introns are unable to form catalytically active structures, as group II introns do, their removal depends on the spliceosomal machinery. Therefore, group II intron splicing is ATPindependent, while the spliceosome-dependent intron excision consumes energy during spliceosome assembly and the catalytically relevant rearrangements (Seetharaman et al., 2006; Lambowitz \& Zimmerly, 2011).

\subsection{Trans-splicing}

As described above, splicing commonly occurs within one RNA molecule and can thus be considered as cissplicing. However, in some organisms, including trypanosomes, nematodes and plants, a rather unusual form of RNA splicing exists, called trans-splicing, in which splicing can occur across two distinct pre-mRNA transcripts (Fig. 1.3).

Cis-splicing

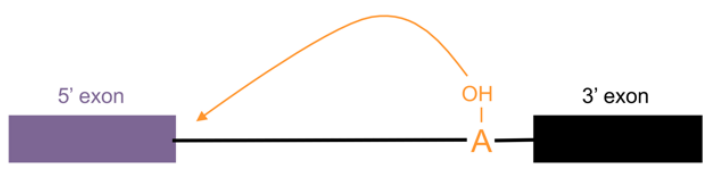

5' SS

BP 3' SS

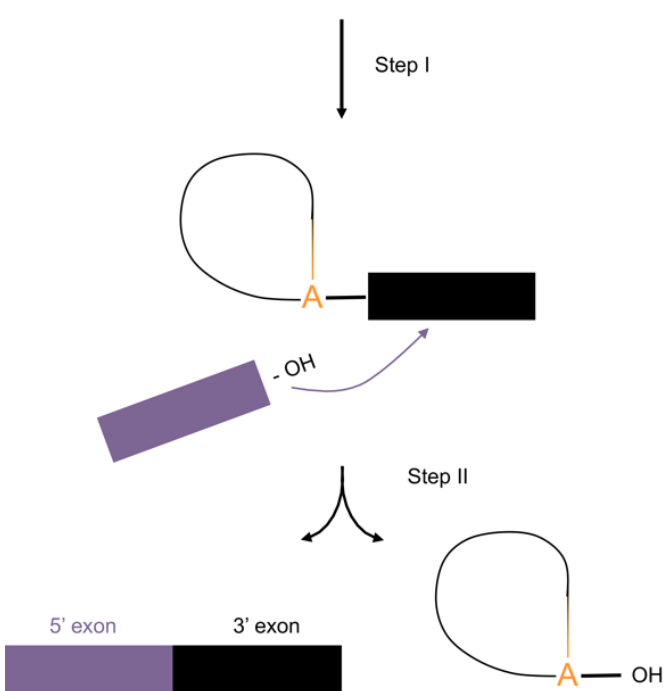

Trans-splicing

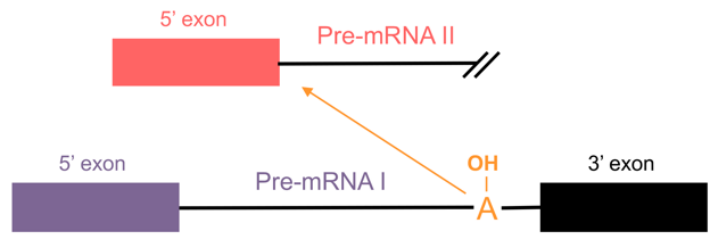

5'SS

BP 3'SS Step I
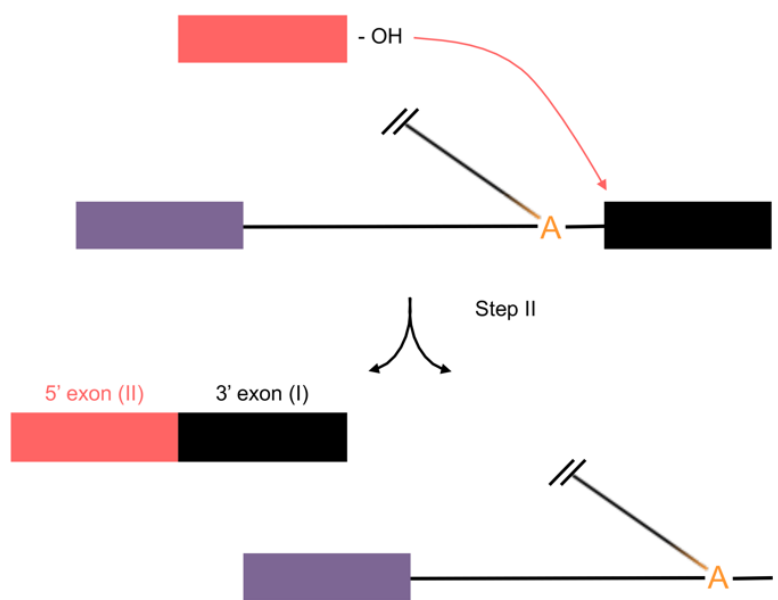

Figure 1.3. Cis-splicing versus trans-splicing. Schematic of the cis-splicing and trans-splicing reactions, both of which comprise two transesterification reactions. While cis-splicing involves only splicing elements from a single transcript, trans-splicing engages splicing signals from two distinct pre-mRNAs, each of which contributes an exon to the mature mRNA. The figure is adapted from (Garcia-Blanco, 2003). 
During this process, the branch point adenosine of the first pre-mRNA (PI) attacks the 5'SS of another pre-mRNA (PII), which liberates the 5' exon of PII and ligates its downstream intron to PI. By attacking the 3'SS of PI, the free 5' exon (PII) is ligated to the 3' exon of PI, combining two exons derived from different transcripts.

Although trans-splicing has recently been described in humans, it appears to be rather seldom. It is unclear why cis-splicing is favored over trans-splicing, but it has been proposed that coupling of splicing to transcription might spatially restrict contacts between different transcripts. However, the mechanism underlying trans-splicing in mammalian cells is poorly understood. (Konarska et al., 1985; Caudevilla et al., 1998; Garcia-Blanco, 2003).

\section{4 snRNPs - the major building blocks of the spliceosome}

The spliceosome is a highly complex molecular machine with a remarkably dynamic structure and composition. The main components of the spliceosome are the small nuclear ribonucleoproteins (snRNPs) U1, U2 and U5 and a U4/U6 di-snRNP (Will \& Lührmann, 2011).

\subsubsection{The small nuclear RNAs}

Each snRNP of the major spliceosome consists of a uridine-rich small nuclear RNA, called snRNA (in the case of the U4/U6 di-snRNP, two snRNAs), that are associated with a defined set of proteins (Lerner \& Steitz, 1979; Bringmann et al., 1984; Hashimoto \& Steitz, 1984). Apart from the U6 snRNA, all snRNAs are transcribed by the RNA polymerase II and thus are equipped with a $5^{\prime}$ terminal $\mathrm{m}^{7} \mathrm{G}$ cap during transcription (Chandrasekharappa et al., 1983; Kunkel et al., 1986). The $\mathrm{m}^{7} \mathrm{G}$ cap not only stabilizes the snRNAs (Furuichi et al., 1977), but also acts as a nuclear export signal (Hamm \& Mattaj, 1990). During maturation of the snRNA in the cytoplasm the survival of motor neuron protein (SMN) complex directs the assembly of the seven Sm proteins B/B', D1, D2, D3, E, F and G in a ring structure around each snRNA's highly conserved uridine-rich Sm-site, forming the so-called Sm-core (Raker et al., 1996; Fischer et al., 1997). This promotes processing of the 3' end of the snRNA and hypermethylation of the $m^{7} G$ cap to a 2,2,7-trimethylguanosine $\left(m_{3} G\right)$ cap, and the premature snRNP is reimported into the nucleus, where additional particle-specific proteins are recruited to form a complete snRNP (Mattaj, 1986; Fischer \& Lührmann, 1990; Nesic et al., 2004).

The U6 snRNA is transcribed by the RNA polymerase III and possesses a rare 5'-terminal $\gamma$-monomethylphosphate cap (Kunkel et al., 1986). This cap increases the stability of the U6 snRNA but does not function as a localization signal (Shumyatsky et al., 1993). As a result, the U6 snRNA remains in the nucleus during maturation (Spiller et al., 2007). The U6-specific equivalent of Sm proteins are the LSm proteins (LSm2-8), which also form a heptameric ring around a uridine-rich sequence at the 3' end of the snRNA (Achsel et al., 1999). After maturation, the U4 and U6 snRNAs form an extensive interaction network, which is followed by association of particle-specific proteins, forming the mature di-snRNP (Wersig et al., 1992). 


\section{Introduction}

The snRNAs form secondary structures, which are highly conserved among higher eukaryotes. Fig. 1.4 illustrates the anticipated structures of the human snRNAs within the respective snRNPs. In the di-snRNP U4 and U6 exhibit complementary regions, which are base-paired to one another (stems I and II) (Brow \& Guthrie, 1988). During the dynamic assembly of the spliceosome some secondary structures, as well as interactions between snRNAs, are remodelled.
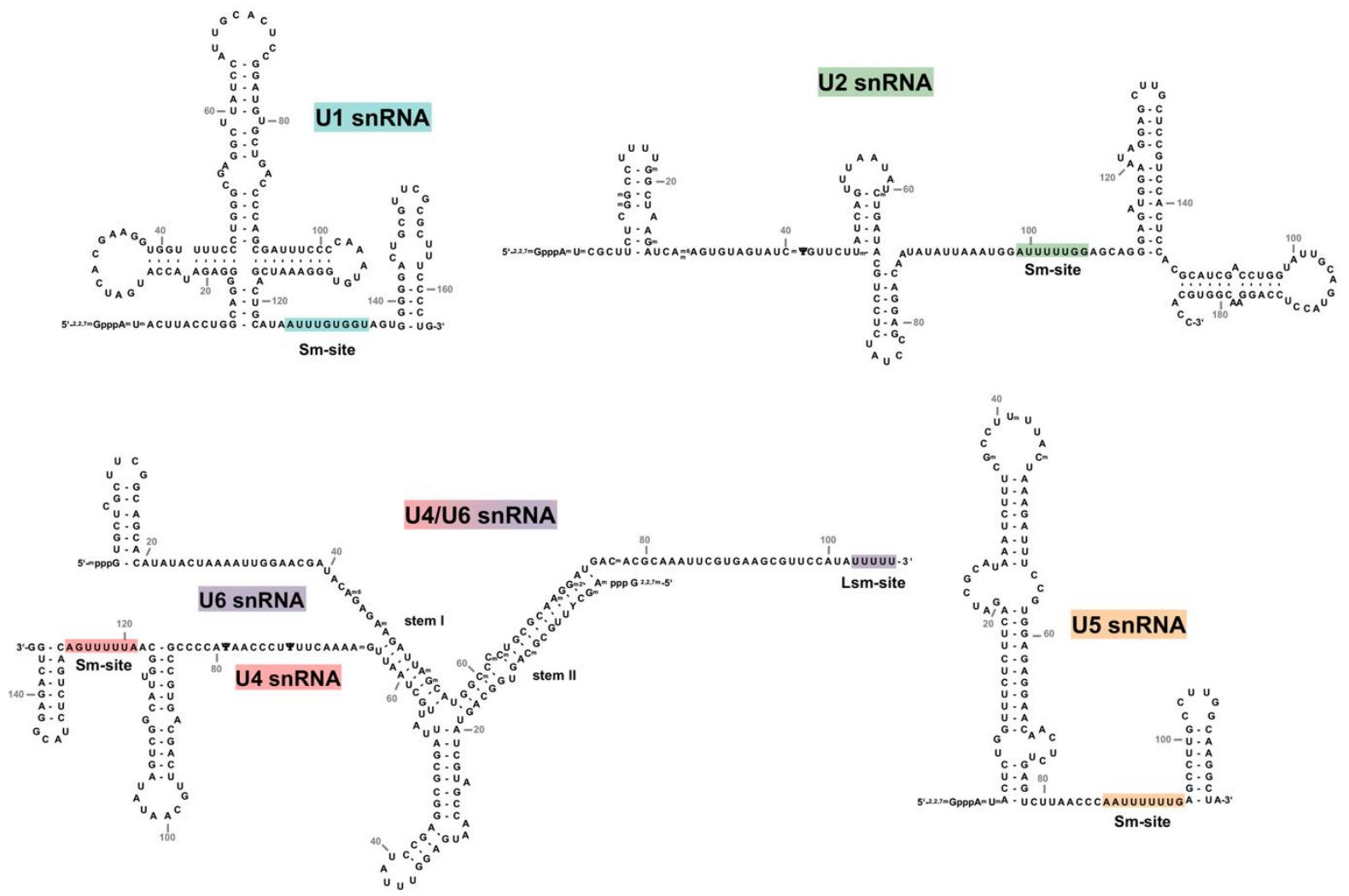

Figure 1.4. Sequences and conserved secondary structures of the human spliceosomal snRNAs. Illustration of the snRNA's structures as anticipated within the respective snRNPs. The basis for this figure was kindly provided by Berthold Kastner Department of Cellular Biochemistry, MPI-BPC.

\subsubsection{The snRNP-specific proteins}

In addition to the Sm or LSm proteins, the snRNAs further associate with a set of snRNP-specific proteins (Fig. 1.5). The $12 S \mathrm{U} 1$ snRNP comprises the particle-specific proteins U1-C, U1-A and U1-70K. Although the U1 snRNA recognizes the 5 'SS by base-pairing, $\mathrm{U} 1-\mathrm{C}$ and $\mathrm{U} 1-70 \mathrm{~K}$ have an important function in stabilizing the interaction of the snRNP with the pre-mRNA (Heinrichs et al., 1990; D. Zhang \& Rosbash, 1999). 
The 17S U2 snRNP comprises the U2-A'/U2-B" dimer and the two multi-protein sub-complexes SF3a and SF3b (Behrens et al., 1993; Brosi et al., 1993). The trimeric SF3a complex is composed of the proteins SF3a120, SF3a66 and SF3a60 and the heptameric SF3b complex includes SF3b155, SF3b145, SF3b130, SF3b49, SF3b14a/p14, SF3b14b and SF3b10 (Will et al., 2002). The SF3a/SF3b proteins are important for A complex formation, as they support the interaction of the U2 snRNA with the BS region (Gozani et al., 1996). Additionally, a number of proteins, called U2-related proteins loosely associate with the U2 snRNP (Will et al., 2002).

The 20 S U5 snRNP comprises six particle-specific proteins (hPrp8, hBrr2, hSnu114, hPrp6, hPrp28, hLin1, 40K and hDib1), some of which are important for functional spliceosome assembly (Bach et al., 1989; Black \& Pinto, 1989; Behrens \& Lührmann, 1991). The two helicases Prp28 and Brr2 are essential for the formation of B and Bact complexes, respectively (Laggerbauer et al., 1998; Staley \& Guthrie, 1999; Boesler et al., 2015). The largest U5 protein, Prp8, is located in the catalytic core of the spliceosome and has been shown to be in contact with the 5'SS, the 3'SS, the BPS as well as the U5 and U6 snRNAs (MacMillan et al., 1994; Umen \& Guthrie, 1995; Reyes et al., 1996). Furthermore, Prp8 interacts with Snu114; both of these have been shown to regulate Brr2 activity (Achsel et al., 1998; Bartels et al., 2002; Small et al., 2006; Maeder et al., 2008; Mozaffari-Jovin et al., 2012).

U1
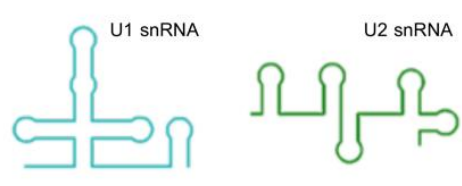

\begin{tabular}{c} 
Sm \\
\hline$A^{\prime}$ \\
$B^{\prime \prime}$ \\
SFa120 \\
SFa66 \\
SFa60 \\
SF3b155 \\
SF3b145 \\
SF3b130 \\
SF3b49 \\
SF3b14a \\
SF3b14b \\
SF3b10
\end{tabular}

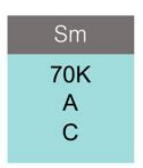

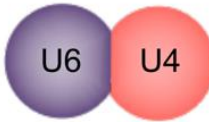

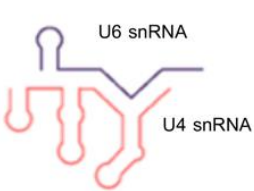

\begin{tabular}{|c|}
\hline LSm/Sm \\
\hline Prp3 \\
Prp31 \\
Prp4 \\
PPIH \\
Snu13
\end{tabular}

U5
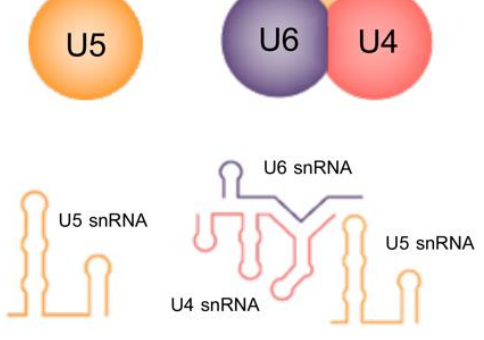

Figure 1.5. Composition of the human snRNPs. Each snRNP consists of one (or two) snRNA(s) and a set of complex-specific proteins. The secondary structure of the human $U$ snRNAs is illustrated schematically. Sm/LSm proteins are shown in grey boxes and the particlespecific proteins of the respective snRNP are shown in the coloured boxes. (Will \& Lührmann, 2011) 


\section{Introduction}

The 13S U4/U6 di-snRNP includes five particle-specific proteins: hPrp3, hPrp31, hPrp4, PPIH and Snu13. The disnRNP is further associated with U5, forming the 25S U4/U6.U5 tri-snRNP. This particle further contains four specific proteins hSnu66, hSad1, 27K and RBM42 (Behrens \& Lührmann, 1991).

While the U4 and U6 snRNAs interact extensively in the di-snRNP, the association of U5 appears to be based rather on protein-protein interactions. The interaction between the U5 protein Prp6 and the di-snRNP protein Prp31 is a prerequisite for U4/U6.U5 tri-snRNP formation (Makarov et al., 2000; Makarova et al., 2002; Schaffert et al., 2004).

\subsection{Spliceosome assembly}

A unique characteristic of the spliceosome is its sequential assembly de novo on each new pre-mRNA substrate (Fig.1.6). None of the snRNPs harbour a pre-formed active site; instead, this is created in a highly controlled manner during the assembly process. Its creation involves substantial remodelling of RNA/RNA and RNA-protein interactions. The driving forces behind these rearrangements are eight highly conserved $D E x D / H-b o x$ ATPases/helicases: UAP56, Prp5, Prp28, Brr2, Prp2, Prp16, Prp22 and Prp43 (Staley \& Guthrie, 1998; Wahl et al., 2009; Will \& Lührmann, 2011).

These helicases belong to the superfamily 2 (SF2) of helicases, which characteristically comprise two RecA-like domains and variable $\mathrm{N}$ - and/or C-terminal regions. The RecA-like domains comprise the highly conserved helicase motifs for binding and hydrolysis of ATP, for binding of RNA and for the coordination of ATP binding and RNA unwinding, respectively (Cordin \& Beggs, 2013; Sloan \& Bohnsack, 2018). During spliceosome assembly, spliceosome activation, splicing catalysis and spliceosome disassembly, these enzymes promote the specific removal of interaction partners (RNA or proteins) in an ATP-dependent manner, which leads to the formation of new base-pairing or protein-binding.

Furthermore, DExD/H-box helicases play a central role in proofreading, i.e., the rejection and discarding of suboptimal substrates, thereby ensuring the high fidelity of splicing (Semlow \& Staley, 2012). While the majority of these enzymes associate only transiently with the spliceosome, the Brr2 helicase is recruited as part of the trisnRNP and is released during spliceosome disassembly (Makarov et al., 2002). Consequently, the extact regulation of Brr2's activity is a prerequisite for preventing premature unwinding of the U4/U6 interaction in the tri-snRNP or the B complex. This task appears to be mainly executed by several other spliceosomal components including the U5 protein Prp8 and probably several B-specific proteins.

Spliceosome assembly is initiated by the binding of the U1 snRNA to the 5'SS of the intron, which is supported by the U1-specific proteins (Zhuang \& Weiner, 1986; Heinrichs et al., 1990; D. Zhang \& Rosbash, 1999). Additionally, SF1, U2AF65 and U2AF35 recognize the BPS, the PY tract and the 3'SS, respectively (Ruskin et al., 1988; Berglund et al., 1997; Merendino et al., 1999).

Already during formation of this first assembly intermediate (the so-called E complex), all relevant splicing elements are recognized by the distinct splicing factors. 
Although the U2 snRNP associates loosely with the E complex, its stable integration into the A complex requires the helicases UAP56 and Prp5 to perform ATP-dependent rearrangements that result in the removal of SF1 from the pre-mRNA and allow the U2 snRNP to interact with the branch-site region (Berglund et al., 1997; Das et al., 2000; M. Zhang \& Green, 2001; Schwer, 2001; Will et al., 2002), whereby the U2 snRNA base-pairs with the branch site (J. Wu \& Manley, 1989; Zhuang \& Weiner, 1989). In addition, numerous U2 SF3a/b proteins establish contacts to $\mathrm{U} 2 \mathrm{AF} 65$ and to the branch-site adenosine itself, as well as to a region upstream of the branch site termed the "anchoring site" (Gozani et al., 1996, 1998; Will et al., 2001; Schellenberg et al., 2006).

This comprehensive interaction network ensures that the splicing motif is identified with high precision. The binding of U2 further causes the branch-site adenosine to bulge out, which is important for the subsequent first step of splicing (Query et al., 1994).

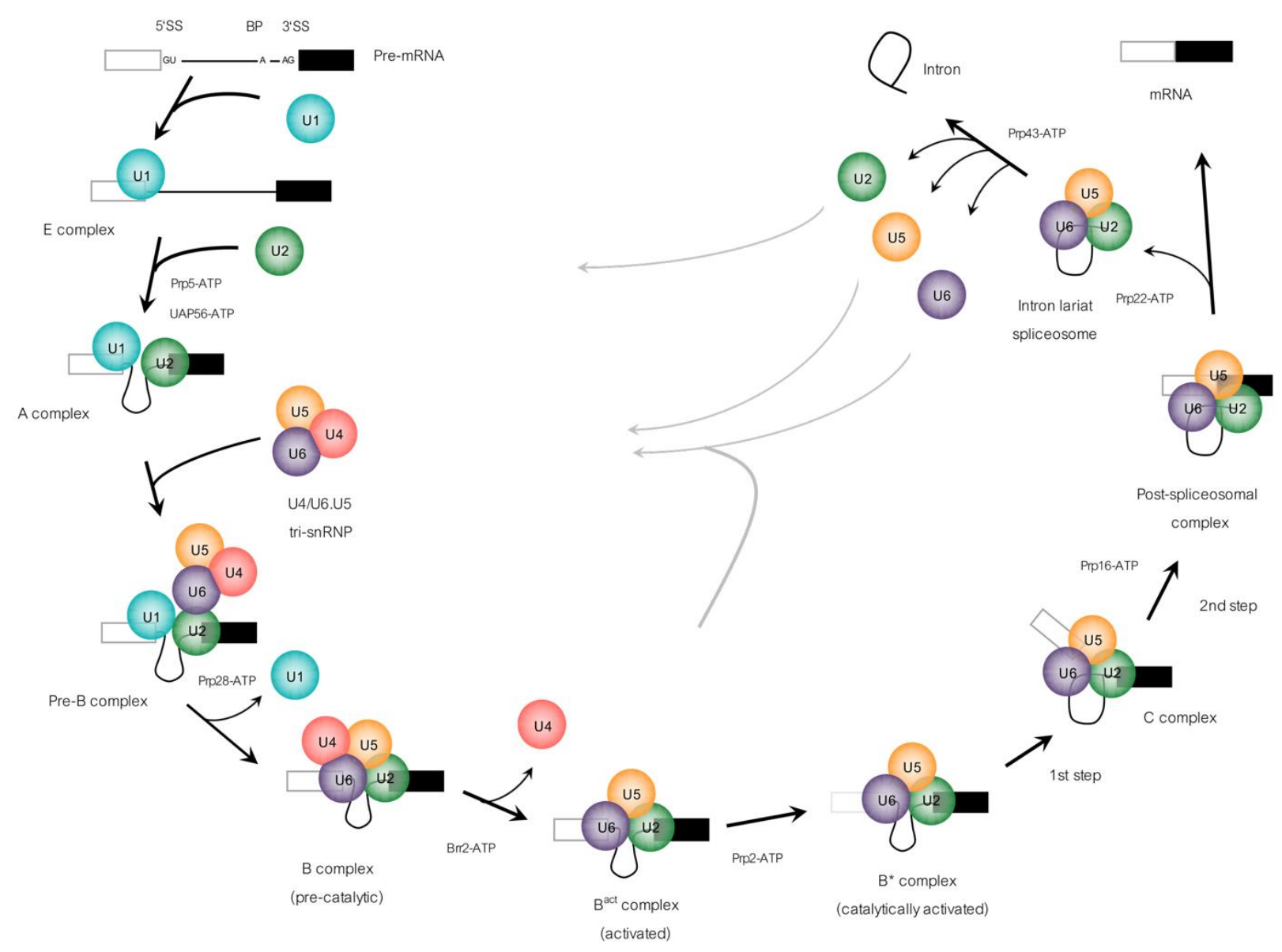

Figure 1.6. Spliceosomal splicing cycle. Schematic diagram of the sequential spliceosome assembly and disassembly during one splicing cycle. The assembly is initiated by the binding of U1 to the 5'SS (E complex). Stable binding of U2 to the BPS results in A complex formation. After loose association of the tri-snRNP (pre-B complex), its stable integration and the loss of U1 lead to the B complex. Structural rearrangements, including the release of $U 4$ ( $B^{\text {act }}$ complex) as well as the subsequent action of Prp2 give rise to a catalytically active $\left(B^{*}\right)$ spliceosome. The first transesterification reaction results in the formation of complex $C$ and the second splicing reaction in the mature mRNA and the intron lariat. The spliceosomal components dissociate and are regenerated for the next round of splicing (modified from Will and Lührmann, 2011 and Boesler et al., 2015). 


\section{Introduction}

Association of the pre-assembled U4/U6.U5 tri-snRNP with the A complex results in the relatively unstable 37S preB complex, which comprises all five snRNAs. In this complex the tri-snRNP is loosely docked to the spliceosome by interacting with U2 forming the U2/U6 helix II. To integrate the tri-snRNP stably into the spliceosome, the binding of the U1 snRNP to the 5'SS is destabilized by the DEAD-box helicase Prp28 and replaced by the ACAGAG box of the U6 snRNA, resulting in the formation of the pre-catalytic B complex (Staley \& Guthrie, 1999; Boesler et al., 2015). At this stage the U5 snRNA establishes contacts to the 5' exon close to the 5'SS, which are maintained throughout both steps of splicing (Sontheimer \& Steitz, 1993; Newman, 2008).

Also, the U5 protein Prp8 is in contact with the pre-mRNA close to the 5'SS, an interaction which is thought to stabilize the U5 snRNA at the 5'SS and appears to be essential for tri-snRNP integration (Teigelkamp et al., 1995; Boesler et al., 2015). Formation of the B complex is further accompanied by phosphorylation of Prp31 (di-snRNP) and Prp6 (U5) through the Prp4 kinase, which is either crucial for or is a result of stable integration of the tri-snRNP (Schneider, Hsiao et al., 2010; Boesler et al., 2015).

To convert the $\mathrm{B}$ complex into an activated spliceosome ( $\mathrm{B}^{\text {act }}$ complex), the $\mathrm{B}$ complex needs to undergo substantial conformational and compositional rearrangements. During activation, the base-pairing of the U4/U6 duplex is unwound by the Brr2 helicase, which results in the release of the U4 snRNA along with the U4/U6-associated proteins and factors that tether the U5 snRNP to the di-snRNP (Laggerbauer et al., 1998; Bessonov et al., 2010). The displacement of U4 allows the U6 snRNA to interact extensively with the U2 snRNA. The simultaneous interaction of U6 with the 5'SS and U2 brings the 5'SS and the BS into close proximity with one another, a conformation that allows the first splicing reaction to take place (Madhani \& Guthrie, 1992; Sun \& Manley, 1995).

However, the $B^{\text {act }}$ complex is still catalytically inactive. As recently shown by cryo-electron microscopy, both the 5'SS and the U2/BS helix are shielded by proteins at this stage. Therefore, the action of the Prp2 helicase is required to promote the destabilization of the U2 SF3b proteins from the BS; it also appears to liberate the 5'SS for catalysis (Lardelli et al., 2010; Ohrt et al., 2012; Rauhut et al., 2016; Haselbach et al., 2018).

The resulting $\mathrm{B}^{*}$ complex is now capable of catalysing the first transesterification reaction, where the branch-point adenosine attacks the 5'SS nucleophilically, thereby liberating the 5' exon and forming the 3' exon-lariat intermediate. This gives rise to the $\mathrm{C}$ complex, which is remodelled by the helicase Prp16 to prepare the spliceosome for the second step of splicing. This includes the displacement of the branch-site region from the catalytic centre and juxtaposition of the 5' exon's 3'-OH and the 3'SS (Schwer \& Guthrie, 1991; Ohrt et al., 2013; Fica et al., 2017).

The resulting $C^{*}$ complex catalyses step two of splicing, during which the 3' hydroxyl group of the 5' exon attacks the 3'SS, resulting in exon ligation and the formation of the intron lariat. The Prp22 helicase then promotes the release of the mature mRNA from the post-catalytic spliceosome (Mayas et al., 2006; Schwer, 2008) in form of a mRNP and is subsequently exported to the cytoplasm (Le Hir et al., 2000). 
The resulting intron lariat spliceosome (ILS) is then disassembled by the action of Prp43. As a result, the intron lariat is released, debranched and degraded, and the snRNPs are separated and recycled to enter another round of splicing (Arenas \& Abelson, 1997; Gee et al., 1997).

\subsection{Gene architecture and pre-mRNA splicing}

The architecture of pre-mRNAs can vary widely between different organisms. In the yeast genome, only $\sim 4 \%$ of all genes include introns, and usually not more than one intron is found per gene. Furthermore, the length of yeast introns is relatively consistent; the vast majority of introns range in length between 50 and 500 bp, with none exceeding 1000 bp (Lopez \& Séraphin, 1999; Spingola et al., 1999).

In contrast, the human genome is much more complex. Around $90 \%$ of all genes include at least one intron, and the average human gene comprises 8.8 exons and 7.8 introns. While exons display a relatively uniform length of $\sim 170 \mathrm{bp}$, the length of introns can vary immensely - from fewer than 100 up to more than one million bases. However, most introns appear to be in the range of 100-1000 bp, while introns with canonical ends are rarely shorter than 70 bases. This sophisticated human genome structure therefore requires many regulatory factors to ensure correct splice site selection and splicing. (Lander et al., 2001; Sakharkar et al., 2004; Abebrese et al., 2017).

\subsubsection{Alternative splicing}

The importance of splicing increases with the complexity of an organism. Whereas splicing is completely absent in prokaryotes, simple eukaryotes, such as yeasts, predominantly make use of basic constitutive splicing, which means that a gene is always spliced in the same way and the product does not show any sequence variation. Complex eukaryotic organisms, such as humans, rely on a more sophisticated form of splicing, called alternative splicing. More than $90 \%$ of human genes containing more than one intron undergo alternative splicing (E. T. Wang et al., 2008; Pan et al., 2008).

Alternative splicing means that the exons of a pre-mRNA can be combined in different ways. In general, five kinds of alternative splicing events are known: (i) cassette exon skipping, (ii) alternative 5'SS or (iii) 3' SS selection, (iv) intron retention and (v) mutually exclusive introns (E. T. Wang et al., 2008; Pan et al., 2008) (Fig. 1.7). This flexibility in intron/exon definition makes possible the generation of several protein isoforms from a single gene, and thus results in a considerable expansion of the cell's protein diversity. By this means the relatively small human genome, which comprises 20,000-25,000 genes, yields an estimated 80,000-120,000 proteins (Liang et al., 2000; Consortium, 2004; Yura et al., 2006). As a result, constitutive and especially alternative splicing account for the greater part of the molecular and cellular complexity in higher eukaryotes (Blencowe, 2006; Y. Wang et al., 2015). 


\section{Introduction}

The regulation of alternative splicing appears to be quite complex and is significantly modulated by trans-acting factors, such as the SR proteins and the hnRNPs. The family of SR proteins is characterized by the so-called RS domain, which comprises long stretches that are rich in the amino acids serine $(S)$ and arginine $(R)$ and promotes the interaction with other protein components. SR proteins further include at least one RNA recognition motif (RRM), by which they bind to pre-mRNAs (Shepard \& Hertel, 2009).

(i) Alternative cassette exons Skipping/inclusion

(ii) Alternative 5' splice site

(iii) Alternative 3' splice site
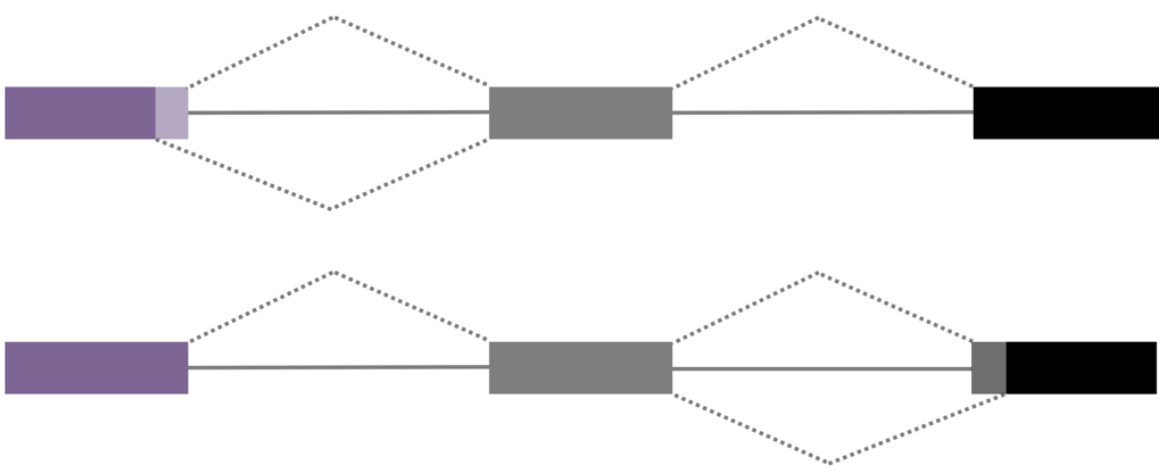

(iv) Intron retention

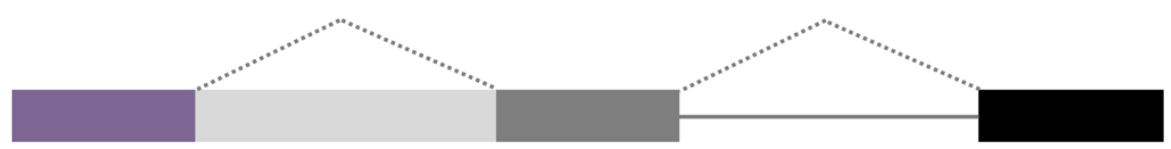

(v) Mutually exclusive exons

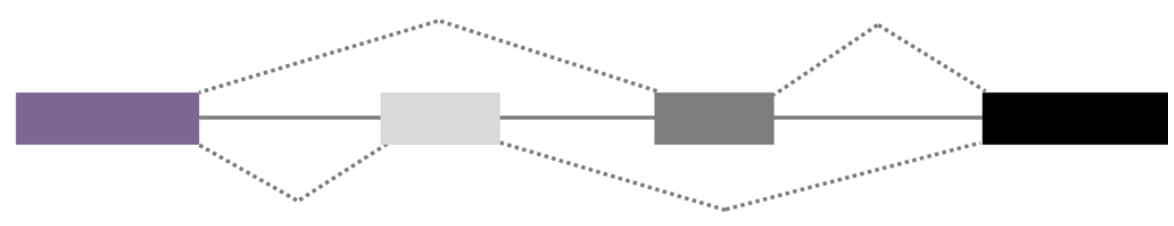

Figure 1.7. Types of alternative splicing. Schematic representation of the different types of alternative splicing. (i) Alternative cassette exons are either included or skipped (omitted). During skipping, the exon and both surrounding introns are excised from the pre-mRNA. Skipping of alternative cassette exons represents the majority of alternative splicing events. (ii \& iii) Selection of an alternative 5' or 3' splice site results in the exclusion of partial exon segments from, or their inclusion in, the mature mRNA. (iv) Intron retention describes the inclusion of an entire intron into the spliced product. (v) When two exons are mutually exclusive, only one of them will be included in the mature transcript. Constitutively spliced exons are indicated as purple and black boxes and introns are shown as solid lines. Alternatively spliced regions are represented by grey boxes and splicing choices are marked by dotted lines. The figure is adapted from Wang et al., 2015. 
The hnRNPs (heterogeneous nuclear RNPs) comprise a more heterogeneous set of nuclear proteins, which specifically associate with primary transcripts of the RNA polymerase II (Geuens et al., 2016). These proteins are structurally more diverse than the SR proteins; typically, they contain several structural domains, of which the RRM domain is the most common motif (Han et al., 2010). Additionally, hnRNPs tend to include auxiliary domains, such as the common RGG repeat, which functions as a protein-protein interaction module to allow interaction with other hnRNPs (Birney et al., 1993).

These splicing factors interact with specific cis-elements that can be located within the intron or the exon. While SR proteins preferentially interact with exonic or intronic splicing enhancers (ESE \& ISE), splicing silencers (ESS \& ISS) are preferentially bound by hnRNPs. The interplay of these elements and splicing factors, which play opposing roles in splicing, are the decisive factors for splice-site choices under given conditions (Black, 2003; Bradley et al., 2014).

\subsubsection{Co-transcriptional splicing}

Although splicing functions independently of transcription, most splicing events occur co-transcriptionally (Beyer \& Osheim, 1988; Goldstrohm et al., 2001; Pandya-Jones \& Black, 2009; Girard et al., 2012).

Pandya-Jones and Black established a cell-fractionation procedure to separate chromatin-associated RNA from soluble nucleoplasmic RNA. Subsequent quantification by quantitative RT-PCR of the nascent RNA fraction and the RNA faction already released showed that the majority of constitutive introns of the c-Src and fibronectin premRNAs were excised while the pre-mRNA was still being transcribed by the RNA Polymerase II. Also introns abutting alternative exons were often removed co-transcriptionally, but with varying excision efficiency among distinct, differentially regulated, cell lines.

This cell-fractionation approach was also applied by Girard et al. to detect co-transcriptional splicing in HeLa cells with an independent readout. They analysed the nucleoplasmic and chromatin fractions by western blotting using antibodies specific for the phosphorylated U2 protein SF3b155 (P-SF3b155). As phosphorylation of SF3b155 occurs only in activated spliceosomes, this antibody thus allows one to detect active spliceosomes in HeLa cells. Quantification of the P-SF3b155 signal revealed that $\sim 80 \%$ of activated spliceosomes were associated with the chromatin, suggesting that only $20 \%$ of pre-mRNA splicing occurs post-transcriptionally.

Coupling of pre-mRNA splicing to transcription not only stimulates both processes (Hirose et al., 1999; Fong \& Zhou, 2001; Millhouse \& Manley, 2005; David et al., 2011), but also improves splicing fidelity and makes possible the coordinated regulation of the three processing events (Neugebauer, 2002; Bentley, 2014).

Co-transcriptional splicing also appears to be an important factor for the regulation of alternative splicing.

Two models have been proposed that may explain how the RNA polymerase II exerts influence on splice site selection; these models are not necessarily mutually exclusive. 


\section{Introduction}

The recruitment model suggests that the C-terminal domain (CTD) of the RNA polymerase II recruits specific splicing factors, including SR proteins and other early spliceosomal factors such as the U1 snRNP, to the nascent transcript, thereby influencing splice-site selection (Misteli \& Spector, 1999; Morris \& Greenleaf, 2000; de la Mata \& Kornblintt, 2006; David et al., 2011). The kinetic model concerns the elongation rate of the RNA polymerase II, which again is determined by certain histone marks. While rapid elongation favours strong splice sites, slow elongation can facilitate spliceosome assembly on introns with weak splice sites or allow binding of splicing enhancers/inhibitors (de la Mata et al., 2003; Dujardin et al., 2014). Since various further factors have been shown to influence alternative splicing it will be a major challenge to understand fully its complex regulation.

\subsubsection{Exon-definition pathway}

In the human genome, intron size can vary from less than $100 \mathrm{nt}$ up to several hundred thousand nt. The spliceosome, however, can only assemble across an intron (intron definition) when the length of the intron does not exceed $\sim 300 \mathrm{nt}$ (Sterner et al., 1996). Therefore, the more frequent longer introns require an alternative to the traditional recognition pathway that allows splice-site identification across the exon (exon definition) (Robberson et al., 1990). In this case, the U1 snRNP interacts with the exon's downstream 5'SS, while the U2 snRNP and U2AF65/35 bind to the BPS or the PY tract/ 3'SS upstream of the exon (Fig. 1.8). SR proteins that bind to ESE sequences support the assembly and stability of the exon-defined A-like complexes (Lam \& Hertel, 2002). Through their RS domains SR proteins can interact with other splicing factors that also include a RS domain, as is the case for U2AF35 and U1-70K. Thus, SR proteins could form an important bridge between the U1 and U2 snRNPs in a cross-exon A-like complex (J. Y. Wu \& Maniatis, 1993; Ram \& Ast, 2006).

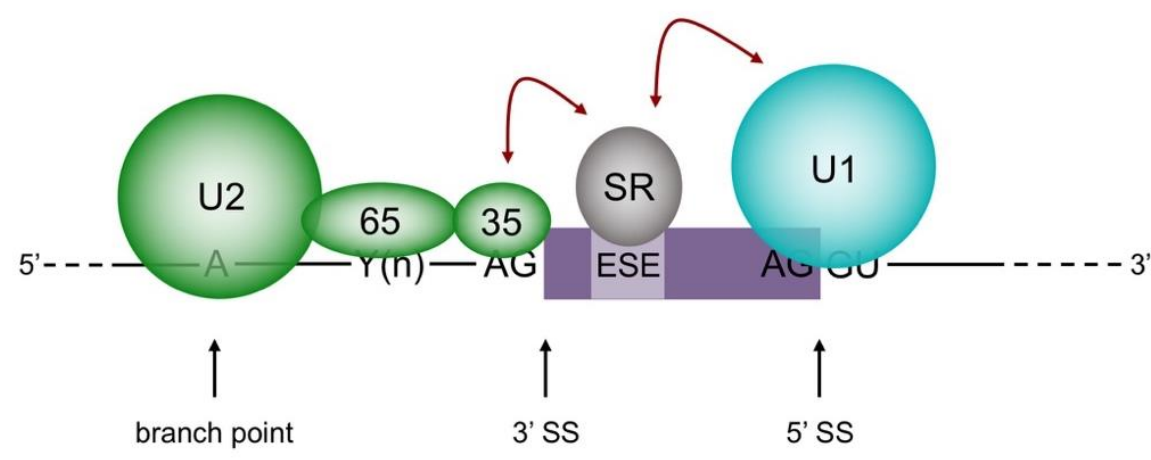

Figure 1.8. Molecular interactions during spliceosome assembly across an exon. In the exon-defined, A-like complex, U2 snRNP is bound to an upstream BPS and U1 snRNP to the downstream 5'SS. The U2AF65/35 heterodimer interacts with the upstream PY tract/3'SS. SR proteins, which bind to exonic splicing enhancers (ESEs), bridge interactions with components of the general splicing machinery (indicated by red arrows). (Ram \& Ast, 2006) 
The splicing reaction, however, can only occur across the intron, and this necessitates the conversion of the exondefined complex to an intron-defined spliceosome. Little is known about the mechanism of this conversion, but, although the commitment to splice-site pairing has been attributed to the formation of the A complex (Lim \& Hertel, 2004), recent studies suggest that exon-defined A-like complexes do not need to undergo a transition to an introndefined A complex. As the U4/U6.U5 tri-snRNP can interact directly with the cross-exon A-like complex, the proximity of an adequate 5'SS allows the subsequent formation of a B-like complex (Fig. 1.9). This complex resembles the common intron-defined $B$ complex and proceeds in the same manner with its catalytic activation and splicing catalysis (Schneider, Will et al., 2010; Boesler et al., 2016).

Numerous studies indicate that the transition to an intron-defined spliceosome is an important checkpoint for determining whether an exon is included or skipped during alternative splice site selection. If an exon cannot be recognized, or the transition to an intron-defined organization is prevented, the exon will inevitably be skipped (Izquierdo et al., 2005; House \& Lynch, 2006; Bonnal et al., 2008; Sharma et al., 2008).

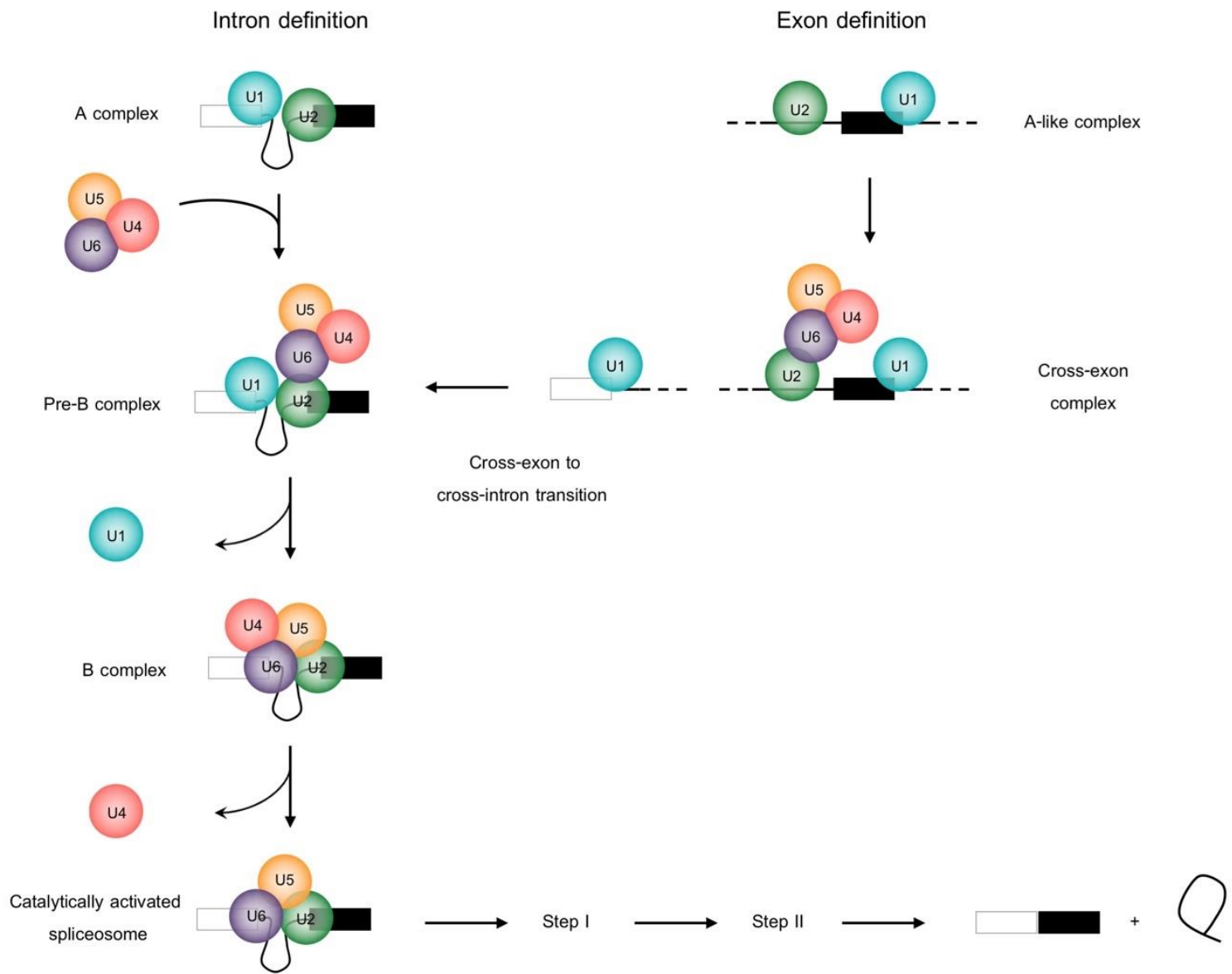

Figure 1.9. Intron- versus exon-definition pathway. Spliceosome assembly during intron and exon definition. In the intron-defined pathway, U1 and U2 snRNPs bind to the 5'SS and BPS of the same intron. Upon association and integration of the U4/U6.U5 tri-snRNP, the B complex is formed. During exon definition, U2 snRNP interacts with an upstream BPS and the U1 snRNP with the downstream 5'SS of the exon. The recruitment of the U4/U6.U5 tri-snRNP results in the cross-exon complex, which makes the transition to an intron-defined organization. Subsequently, spliceosome assembly proceeds along the canonical splicing pathway. The figure is adapted from Schneider, Will et al., 2010. 


\section{Introduction}

\subsubsection{Spliceosome assembly requires a minimum intron length}

Intron length represents an important factor in pre-mRNA splicing. While the alternative exon-definition pathway enables the spliceosome to deal with introns that exceed the acceptable size for intron definition, a minimum intron length appears to be a prerequisite for the assembly of functional spliceosomes. Investigation of short introns in human cells showed that canonical introns, which are thought to be excised by the spliceosome, are rarely shorter than $70 \mathrm{nt}$ (Abebrese et al., 2017). Consistently, in the 1980s several studies showed that a minimum of 60-80 nt must separate the 5'SS and the 3'SS to allow proper splicing (Wieringa et al., 1984; Ulfendahl et al., 1985). Soon after this discovery, other studies defined the decisive factor more precisely as the distance between the 5'SS and the branch site, as elongating the intron by PY-tract extension could not restore splicing (Fu et al., 1988; Köhrer \& Domdey, 1988; Smith \& Nadal-Ginard, 1989). A distance between the 5'SS and the BS of 45-52 nt was shown to result in the selection of an upstream cryptic 5'SS, which increases the distance to the branch site. In the absence of an alternative splice site, the intron was shown to be retained in the mature mRNA (Krainer et al., 1984; Köhrer \& Domdey, 1988).

Steric hindrance has been suggested as a possible reason for a minimum length requirement. The U1 and (especially) the U2 snRNP are large complexes, whose simultaneous binding to the 5'SS and to the BS may require a certain amount of space and flexibility (Wieringa et al., 1984; Fu et al., 1988). This idea has been supported by native gel and sedimentation analysis, which showed impaired spliceosome assembly on short non-functional introns (Köhrer \& Domdey, 1988; Smith \& Nadal-Ginard, 1989; Himmelspach et al., 1991). Thus, intron size seems to be a relevant determinant for spliceosome assembly and splicing. However, the exact mechanism of intronlength-dependent spliceosome assembly is only poorly understood.

\subsection{Dynamic RNA/RNA interaction network}

The formation of a functional spliceosome is associated with extensive remodelling of the RNA/RNA interaction network (Fig. 1.10) (Wahl et al., 2009; Will \& Lührmann, 2011). The initial recognition of the 5'SS occurs through base-pairing with the 5 ' end of the U1 snRNA (Zhuang \& Weiner, 1986). The second interaction with the pre-mRNA is formed by the U2 snRNA, resulting in a short U2/BPS-duplex, where the branch-point adenosine is bulged out (J. Wu \& Manley, 1989; Zhuang \& Weiner, 1989; Query et al., 1994). This exposes the adenosine's 2' hydroxyl group for nucleophilic attack at the 5 'SS.

Upon recruitment of the U4/U6.U5 tri-snRNP, the 5' end of the U2 snRNA and the 3' end of the U6 snRNA form a short helix (U2/U6 helix II). Stable integration of the tri-snRNP, however, involves the displacement of U1 from the 5'SS and also base-pair formation between the 5'SS and the U6 ACAGAG box. In the B complex U6 is thus basepaired to U2, to the pre-mRNA and extensively to U4. (Staley \& Guthrie, 1999; Boesler et al., 2015). 
The U6 snRNA includes important components of the catalytic centre, which are kept in an inactive state by hybridization with the U4 snRNA in the tri-snRNP and the B complex to prevent premature catalysis. Consequently, the catalytic activation of the spliceosome requires the disruption of the U4/U6 snRNA duplex (U4/U6 stem I and stem II), which is followed by release of the U4 snRNP (Laggerbauer et al., 1998; Raghunathan \& Guthrie, 1998). This allows U6 to form interactions with the U2 snRNA (U2/U6 helix la and I), thereby juxtaposing the 5'SS and the BPS for the first splicing reaction. The release of U4 further allows U6 to form an internal stem-loop (U6 ISL) that structurally resembles domain $\mathrm{V}$ (D5) of group II self-splicing introns and, likewise, coordinates catalytically important metal ions (Madhani \& Guthrie, 1992; Anokhina et al., 2013).

Cryo-electron microscopy of the first $S$. pombe spliceosome illustrated the major structural similarities between the U6 ISL and D5 (Hang et al., 2015). In the spliceosome, three conserved motifs are involved in metal-binding. These include (i) the so-called catalytic triad AGC, which is located within the U2/U6 helix lb at the stem of the U6 ISL, (ii) a bulged-out uridine located five nucleotides from this triad and (iii) the U6 ACAGAG box. These three structures formed by $\mathrm{U} 2$ and $\mathrm{U} 6$ are important for the RNA-metal-mediated catalysis of both pre-mRNA splicing steps (Keating et al., 2010; Fica et al., 2013). Thereby, the catalytic AGC triad forms a triple helix with the terminal GA of the ACAGAGA sequence and the bulged-out uridine of the U6 ISL. This so-called catalytic triplex coordinates two magnesium ions at a distance of about $4 \AA$; these mediate the catalysis of both steps of pre-mRNA splicing. In the same manner, magnesium ions are coordinated by group II self-splicing introns, suggesting that group II introns and the spliceosome share an evolutionary heritage.

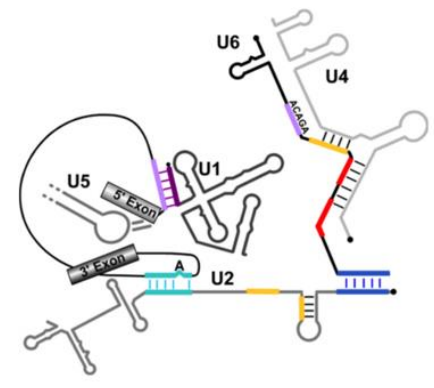

37 S pre-B complex

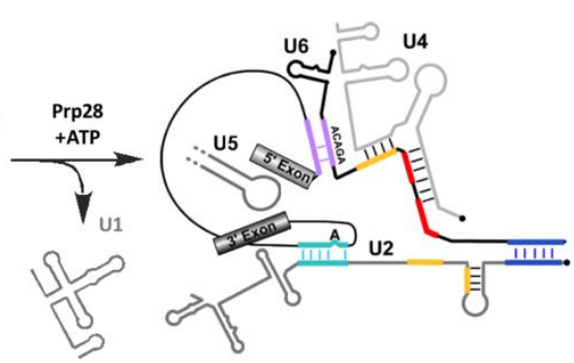

pre-catalytic spliceosome (B complex)

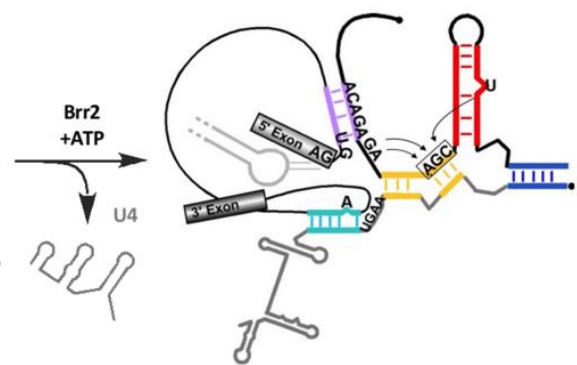

activated spliceosome (Bact complex)

Figure 1.10. Dynamic RNA-RNA network in the spliceosome. Schematic illustration of the rearrangements of RNA-RNA interactions during formation of a catalytically activated spliceosome. In the pre-B complex, the U1 snRNA is base-paired with the 5'SS (aubergine), and the U2 snRNA is bound to BPS (turquoise). Initial association of the U4/U6.U5 tri-snRNP occurs between the U6 and U2 snRNAs (blue). Stable integration of the U4/U6.U5 tri-snRNP during B complex formation involves the disruption of base-pairing between the U1 snRNA and the 5'SS, which allows the 5'SS to interact with the conserved ACAGAG motif of the U6 snRNA (purple). During activation, the base-pairing between U4 and U6 snRNAs is disrupted, resulting in extensive base-pairing between the U2 and U6 snRNAs via helix (yellow). The U6 snRNA further forms an essential internal-stem loop (ILS) (red), while the U2 snRNA remains base-paired with the BPS. U5 snRNA contacts nucleotides of the exon. Exons are represented as grey boxes, while introns are shown as a black line. Secondary structures of snRNAs are shown schematically in grey or black. Of the U5 snRNA, only loop I is shown. The regions involved in basepairing (short lines) are shown in different colours. This figure was kindly provided by Berthold Kastner, Department of Cellular Biochemistry, MPI-BPC. 


\section{Introduction}

The U5 snRNA comprises a stem loop (U5 stem loop I), which interacts with the 5' exon before and after the first step of splicing and further contacts the 3' exon after the first catalytic step has occurred. Thus, the U5 snRNA plays an important role in properly positioning the two exons for the second splicing reaction (Sontheimer \& Steitz, 1993; Newman, 2008).

\subsection{Dynamic protein composition of the spliceosome}

The spliceosome is a particularly protein-rich molecular RNP machine. Around 170 proteins have been identified as spliceosomal components in humans, while individual spliceosomal complexes can contain $\sim 110$ proteins (Fig. 1.11). The yeast spliceosome comprises fewer ( 90) protein factors (Fabrizio et al., 2009), most of which have homologues in higher eukaryotes. This indicates that the yeast spliceosome represents the basic, evolutionarily conserved core spliceosome. Consistently with this idea, it appears that many proteins that are not conserved from yeast to human have regulatory roles, such as the regulation of alternative splicing.

In addition to the $\sim 45$ snRNP-associated factors, a number of non-snRNP proteins accompany the spliceosome. Their attachment is often transient and limited to the stages when their presence or action is required. (Wahl et al., 2009; Will \& Lührmann, 2011).

Mass-spectrometric analysis of spliceosomal complexes assembled in vitro have allowed the determination of the protein composition of the spliceosome's major assembly stages, thereby revealing the extent of protein exchange between one intermediate and the next. This in turn allowed the identification of groups of proteins that are exclusively present at each particular stage, thereby giving a unique proteomic signature to distinct intermediate spliceosomal complexes (Deckert et al., 2006; Behzadnia et al., 2007; Bessonov et al., 2010; Agafonov et al., 2011).

During B complex formation a number of designated A complex proteins and U1 snRNP proteins are released, while about 25 proteins join the spliceosome as part of the U4/U6.U5 tri-snRNP together with a number of nonsnRNP protein factors. These include a group of proteins, previously identified as B-specific proteins, as well as the pre-assembled Prp19/CDC5L complex, a set of Prp19/CDC5L-related proteins and the RES complex. Owing to their sub-stoichiometric abundance in the B complex, the latter spliceosomal factors were thought be only loosely associated with the B complex, with their stable integration following during the activation. However, recent evidence suggests that these proteins are recruited only after $B^{\text {act }}$ complex formation.

This protein composition was based on the analysis of so-called kinetic B complexes, which were obtained by incubation of the pre-mRNA under splicing conditions for 5-8 minutes before the assembly was stopped. During this time no first-step products are formed, indicating that the majority of spliceosomes are in a pre-catalytic state. Nevertheless, when B complexes are kinetically stalled the possibility exists that a small fraction of the population undergoes activation, which can give a false impression of the actual situation. 
Introduction
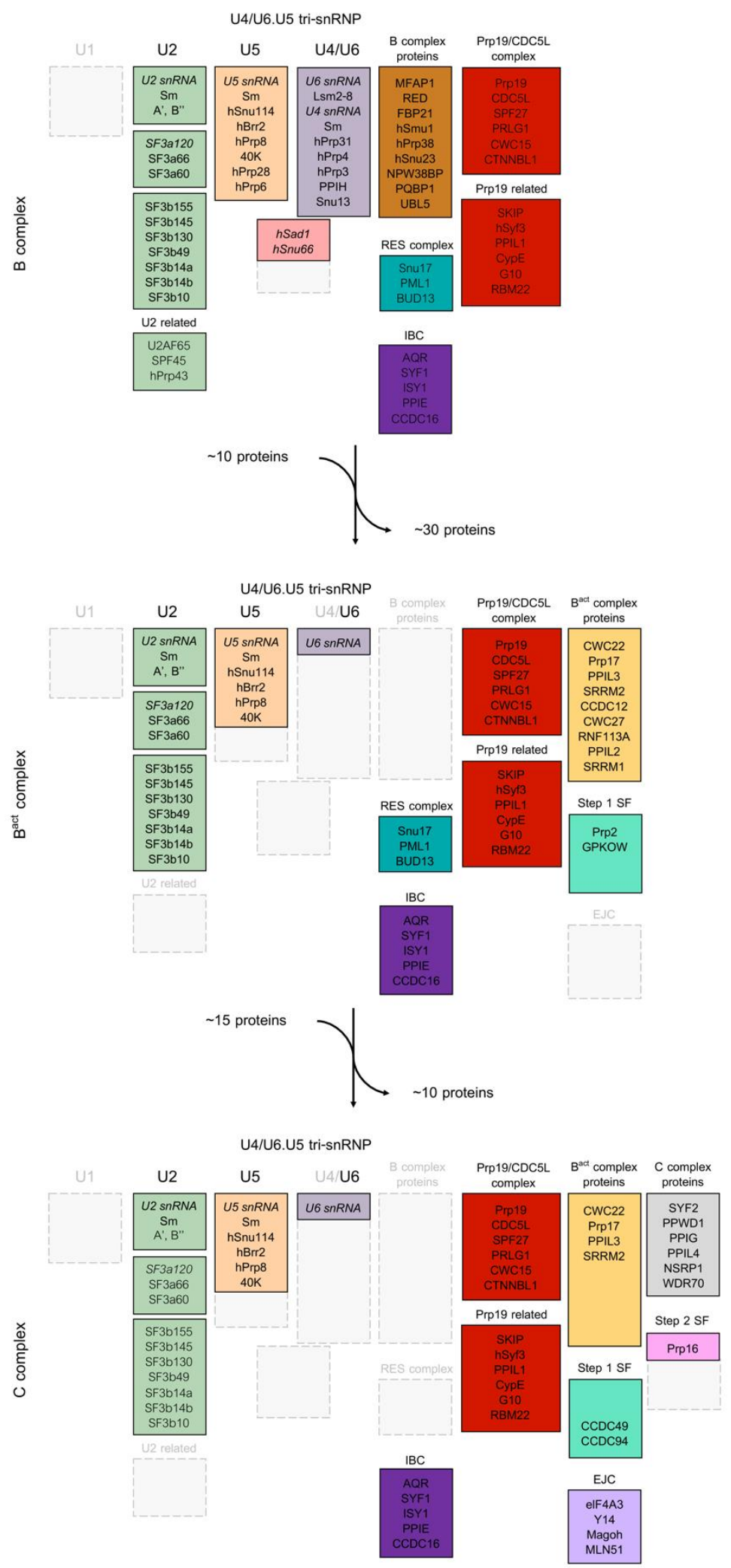

Figure 1.11. Dynamic protein composition of the human spliceosome. The protein composition of the human $B, B^{\text {act }}$ and $C$ complexes as determined by $2 \mathrm{D}$ gel-electrophoresis and mass spectrometry. The $\mathrm{C}$ complex was stalled before the Prp16-driven rearrangements by using a dominant negative Prp16 mutant. The relative abundance of proteins is indicated by bold (stoichiometric amounts) or light (substoichiometric amounts) lettering. Proteins are grouped according to their snRNP association, function, presence in a stable heteromeric complex or association with a particular spliceosomal complex, as indicated. SR and hnRNP proteins, as well as those present in very low amounts, are not shown. This figure is adapted from results kindly obtained by Leyla El Ayoubi and Dmitry Agafonov, Department of Cellular Biochemistry, MPI-BPC. 


\section{Introduction}

Recently, two studies showed that when B complexes were stalled with the ATP analogue ATPyS (B ${ }^{A T P S}$ ) (Agafonov, Van Santen et al., 2016) or by lowering the concentration of $\mathrm{MgCl}_{2}\left(\mathrm{~B}^{\text {DeltaMgCl2}}\right)$ (Bertram, Agafonov, Dybkov et al., 2017) the Prp19/ CDC5L complex, the Prp19-related proteins as well as the RES complex were essentially absent; only RBM22 and SKIP were present in sub-stoichiometric amounts. In contrast, the B-specific proteins were stoichiometrically present. This suggests that in the purified the kinetic B complexes the PRP19/CDC5L and Prp19-related proteins may be derived from contaminating $\mathrm{B}^{\text {act }}$ complexes, whereas the stalled B complexes represent a more homogeneous B complex population.

The exclusive association of the B-specific proteins with the B complex led to the initial assumption of a role for them in B complex formation. However, recent studies by Boesler et al. have suggested that these proteins are not necessarily required for stable B complex formation in vitro (Boesler et al., 2015, 2016).

The subsequent activation of the spliceosome is also highly dynamic at the level of protein composition. Proteins associated specifically with the U4/U6 di-snRNP and the U4/U6.U5 tri-snRNP, as well as the B-specific proteins,

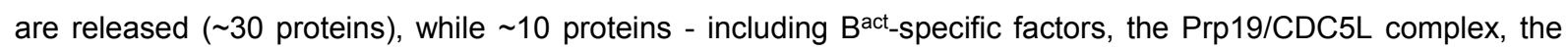
inron-binding complex (IBC) and the RES complex - are stably integrated and are present in stoichiometric amounts in the $B^{\text {act }}$ complex. While the Prp19/CDC5L complex was proposed to be essential for spliceosome activation and/or the first step of splicing (Makarova et al., 2004), little is known about the function of the RES complex in splicing. A recent study suggests that in yeast this complex is involved in the B-to-Bact transition (Bao et al., 2017). However, while these proteins are already associated with the yeast B complex, they appear to be recruited to the human spliceosome after its activation.

During $\mathrm{C}$ complex formation, only moderate structural changes take place, but $\sim 10$ proteins, the presence of which is no longer required, are released while the $C$ complex-specific proteins, the first-step splicing factors and members of the exon junction complex (EJC) are recruited ( 15 proteins). The EJC is placed onto the pre-mRNA upstream of the splice junction and remains associated with the mature mRNA to modulate its downstream metabolism in the cell (Le Hir \& Séraphin, 2008).

Finally, the second-step factors and a group of putative $C^{*}$-specific proteins join the spliceosome after the first step of splicing, while some $\mathrm{C}$ complex proteins are released.

\subsection{Post-translational phosphorylation of proteins during splicing}

Besides the dramatic compositional and conformational changes that the spliceosome undergoes during splicing, alterations in the phosphorylation state of distinct spliceosomal proteins have also been recorded.

Reversible protein phosphorylation is the most common post-translational modification, and the phosphorylation and dephosphorylation of certain proteins has been shown to be essential for spliceosome assembly and splicing catalysis. Interestingly, the majority of spliceosome-related phosphorylation events identified so far take place on serine/threonine residues. At the beginning of the splicing cycle, for example, SR proteins are intensively 
phosphorylated (Xiao \& Manley, 1997) and several snRNP-associated proteins undergo phosphorylation in early stages of the splicing cycle. This includes extensive phosphorylation of the U1 snRNP protein 70K during E complex formation; this phosphorylation step has been shown to be essential for spliceosome assembly (Woppmann et al., 1993; Hernández et al., 2009).

Additionally, B complex formation is accompanied by phosphorylation events, such as that of Prp28 (by SRPK2), Prp31 (by Prp4-kinase; Fig. 1.12) and Prp6 (also by Prp4-kinase). These proteins were shown to be phosphorylated during stable integration of the tri-snRNP (Schneider, Hsiao et al., 2010).

Furthermore, the U2-associated protein SF3b155 is phosphorylated at specific serine/threonine residues in the Bact complex before the first catalytic step and is subsequently dephosphorylated in the $\mathrm{C}$ complex before the second step of splicing (Agafonov et al., 2011; Girard et al., 2012).

The two redundant phosphatase families PP1 and PP2A were identified as mediating splicing catalysis by dephosphorylating their main substrates, phosphorylated U2-SF3b155 and U5-116K, respectively, which is relevant for initiating the second catalytic splicing step. Similarly, dephosphorylation of $70 \mathrm{~K}$, which already occurs during $\mathrm{A}$ complex formation, is a prerequisite for the first splicing step to occur (Shi et al., 2006; Wahl et al., 2009).

In general, it can be said that phosphorylation occurs predominantly during spliceosome assembly and activation, whereas catalysis and disassembly are rather characterized by dephosphorylation events. At present it is unclear how the phosphorylation/dephosphorylation of specific proteins controls the progression of the splicing cycle. However, it is believed that phosphorylation events are likely to exert control over protein-protein interactions.

\subsection{The structure of the human B complex and the organization of the B-specific proteins}

While the composition of the spliceosome has been investigated extensively over many years, it is only recently that detailed structural information could be provided. The size of the spliceosome and its flexibility render comprehensive structural analysis challenging. In the past years, however, several high-resolution structures of distinct spliceosomal complexes derived from yeast and human have been resolved by cryo-electron microscopy (cryo-EM). These include the tri-snRNP (Nguyen et al., 2015, 2016; Agafonov, Kastner et al., 2016), the B complex (Bertram, Agafonov, Dybkov et al., 2017; Plaschka et al., 2017; Bai et al., 2018), the Bact complex (Rauhut et al., 2016; Yan et al., 2016; Haselbach et al., 2018; X. Zhang et al., 2018), the C complex (Galej et al., 2016; Wan et al., 2016; Zhan et al., 2018), the C* complex (Bertram, Agafonov, Liu et al., 2017; Fica et al., 2017; Yan et al., 2017), the P complex (Bai et al., 2017; Liu et al., 2017; Wilkinson et al., 2017) and the ILS (Wan et al., 2017). Together, these structures have provided a wealth of information about the location of RNAs and proteins in the various spliceosomal "snapshots" and their dynamics during the transition from one complex to the next. 


\section{Introduction}

Bertram, Agafonov, Dybkov, et al. (2017) were able to resolve the structure of the human B complex (Fig. 1.12A), which also revealed the locations of several domains of the B-specific proteins. In the human B complex the U2containing globular head domain is found on top of a triangular body; the latter comprises the tri-snRNP and the Bspecific proteins. The two domains are connected by three main bridges. Bridge B1 comprises the U2/U6 helix II, B2 includes spliceosomal proteins and B3 most likely contains the protein-bound intron, which interacts with both the $\mathrm{U} 2$ and $U 6$ snRNAs.

As observed for the human $B^{\text {act }}$ (Haselbach et al., 2018; X. Zhang et al., 2018) and C* complexes (Bertram, Agafonov, Liu et al., 2017), the intron forms extended helices with the U6 ACAGAG box at the 5'SS and U2 at the BS. These helices extend beyond the short 5'SS or BS regions and are thought to stabilize the interaction of the snRNAs with the pre-mRNA in the spliceosome, which is further supported by the binding of protein factors.

The U4 Sm core and helix I of the U4/U6 duplex are connected by a single-stranded region of U4, which serves as a docking site for the N-terminal helicase cassette of Brr2 and is essential for the helicase's U4/U6 duplex-unwinding activity in vitro (Mozaffari-Jovin et al., 2012). In the B complex, the Brr2 helicase is loaded onto this single-stranded region of the $U 4$ snRNA, but it is not yet in contact with the U4/U6 duplex region. As this isolated $B$ complex remains stable in the presence of ATP, the helicase activity of Brr2 is likely to be negatively regulated in this complex. It appears that at least some of the B-specific proteins are involved in the regulation of Brr2 in the spliceosome when it is primed for activation, which makes their position in the B complex particularly interesting (see below).

During formation of the B complex, the Prp28 helicase is destabilized, which allows the three B-specific proteins Prp38, MFAP1 and Snu23 to bind as a trimeric protein complex close to the extended U6 ACAGAG/5' SS helix. Although it has been suggested that Prp38 and MFAP1 are important for a functional activation step, the underlying mechanism is poorly understood (Lybarger et al., 1999; Schütze et al., 2016; Ulrich \& Wahl, 2017). The structure of the human B complex shows that the NTD of Prp38 as well as known $\alpha$ - helices of Snu23 and MFAP1 are in direct contact with the U6 ACAGAG/5'SS helix region (Fig. 1.12B). Therefore, involvement in repositioning the 5'SS during activation has been proposed for these proteins.

FBP21 is another member of the set of B-specific proteins. It is conserved among the higher eukaryotes but does not have any known counterpart in yeast. In the human B complex FBP21 appears to bind through its zinc finger domain to the minor groove of the U6 ACAGAG/5'SS helix and also to contact Brr2 (Fig. 1.12B), suggesting that FBP21 might be important for maintaining Brr2 in an inactivate state in the B complex. Consistently with this, a recent study suggested that the helicase activity of Brr2 is inhibited in the presence of FBP21 (Henning et al., 2017). Interestingly, in spliceosomal complexes stalled with the small-molecule inhibitor cp028 (designated as the B028 complex), FBP21 is the only B-specific protein that is absent (Sidarovich et al., 2017). This compound inhibits spliceosome maturation at an intermediate state after the release of the U4 snRNP, but before the release of the LSm and B-specific proteins and the recruitment of the Prp19/CDC5L complex, the Bact-specific proteins etc. It appears that with the release of FBP21 a physical barrier is removed, which makes possible the Brr2-driven activation of the spliceosome. 
Taken together, these results suggest that FBP21 prevents premature unwinding of the U4/U6 interaction by inhibiting Brr2 activity in early B complexes. This essential role of FBP21 in the regulation of spliceosome assembly underlines both the importance of the precise control of Brr2 and the possibility that splicing factors specific for higher eukaryotes may be indispensable for basic splicing.

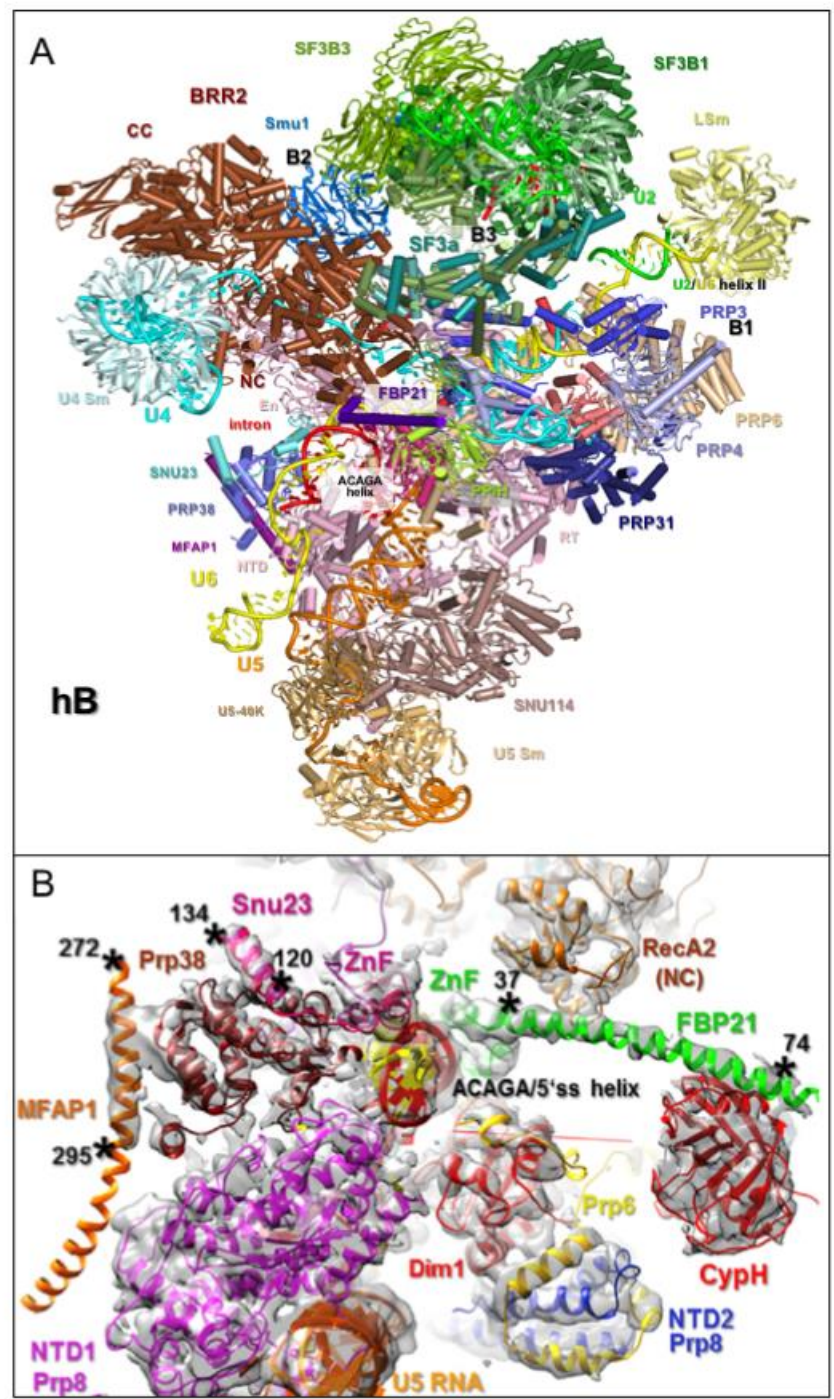

Figure 1.12. Structure of the human B complex. (A) Cryo-eletron microscopy structure of the human $B$ complex. Green proteins and snRNA belong to the U2 head domain. Proteins and snRNAs with other colours are part of the tri-snRNP or belong to the B-specifc proteins. The three bridges are indicated by black letters. The proteins are labelled in the corresponding colours. (B) Locations of the Bspecific proteins Prp38, Snu23, MFAP1 and FBP21 within the human B complex as determined by cryo-electron microscopy. This part of the figure was taken from Bertram, Agafonov, Dybkov et al., 2017 


\section{Introduction}

Like FBP21, Smu1 and RED are found in higher eukaryotes while being absent in yeast. These two proteins have been the targets of several studies, yet their function in splicing is poorly understood. Owing to their absence in simple organisms, it has been proposed that they may act as regulatory factors rather than having an essential core function. This is supported by a recent study that showed that siRNA-mediated depletion of Smu1 or RED changes the pattern of alternative splicing in human cells (Papasaikas et al., 2015). Interestingly, alternative splicing appeared to be affected in a very similar manner by siRNA-mediated knock-down of Smu1 or RED, suggesting that these two proteins function as a single unit. Consistently with this, several other studies have provided evidence for a physical and functional connection between Smu1 and RED. For instance, a Smu1-RED complex was shown to be recruited by the influenza virus RNA polymerase whose transcripts appeared to be spliced differently upon depletion of RED/Smu1 (Fournier et al., 2014). Furthermore, the siRNA-induced depletion of either Smu1 or RED in cells has been shown to result in co-depletion of the other, suggesting that the interaction between these two proteins improves the stability of both of them (Spartz et al., 2004).

A
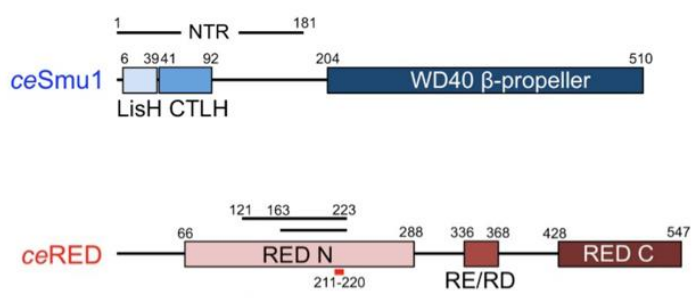

B

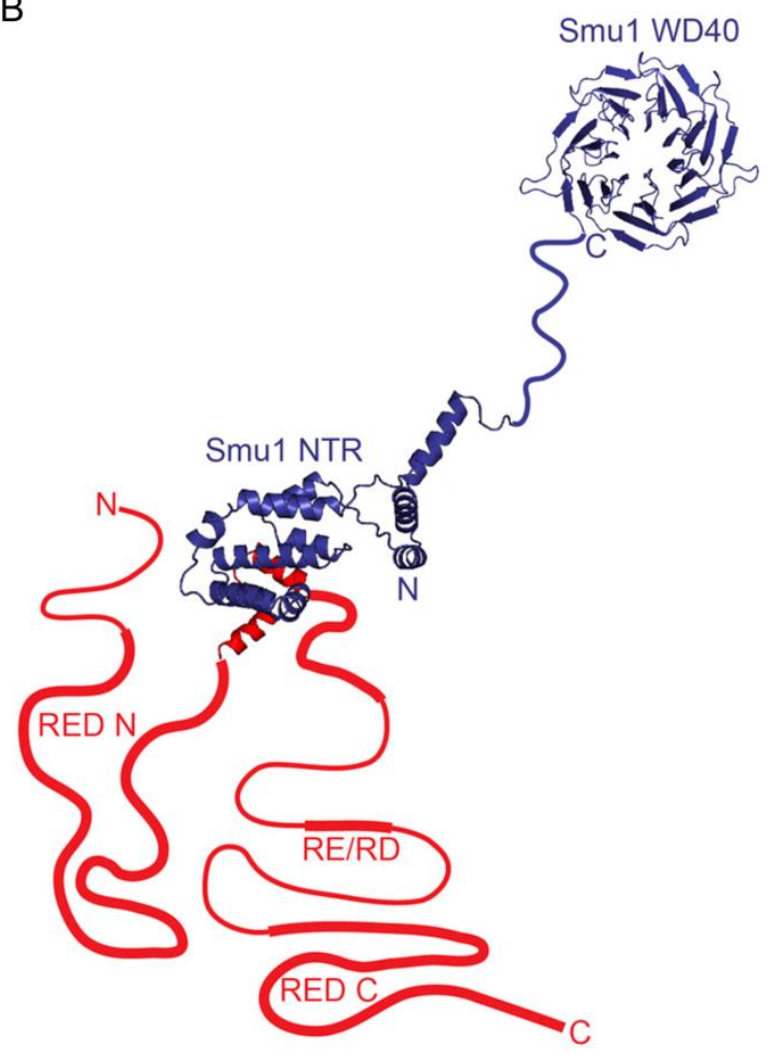

Figure 1.13. Structure of C. elegans Smu1 and RED. (A) Schematic illustration of Smu1 and RED from C. elegans. (B) The structures of the NTR of Smu1 and the short helices of RED that interact with Smu1 were determined by crystallography. The intrinsically disordered protein RED is illustrated schematically and a common folding of the Smu1-WD40 is shown. (Ulrich et al., 2016) 
Yeast two hybrid and structural studies further proposed that Smu1 and RED form a functional module that recruits further spliceosomal factors to the spliceosome (Hegele et al., 2012). Apart of a LisH-CTLH homo-dimerization motif and a globular core domain, which is involved in hetero-dimer formation with RED, Smu1 further comprises a C-terminal WD40 domain. This domain is known to mediate protein-protein interactions and was thus suggested to serve as a binding platform for other proteins. RED, which is intrinsically disordered, was shown to form a short helical structure only upon interaction with Smu1 and was proposed to contact additional spliceosomal proteins in a similar manner (Fig. 1.13) (Ulrich et al., 2016).

The cryo-EM structure of the human B complex has indicated a new possible role for Smu1 and RED - that is, in forming a bridge between U2 and the tri-snRNP in the B complex (Bertram, Agafonov, Dybkov et al., 2017). Specifically, Smu1 directly contacts the WD40 domain 2 of U2-SF3B3 and the C-terminal cassette of Brr2 through its WD40 domain (Fig. 1.12A). It is possible that this interaction stabilizes the position of Brr2 relative to its U4/U6 substrate and might be important during the activation. The precise location of RED in the B complex could not be determined since this intrinsically disordered protein lacks clear structural domains. However, chemical crosslinking experiments suggest that RED makes contact with several U2 proteins and with Smu1 as well as with Prp8. This shows that RED and Smu1 engage in several contacts within the spliceosome and may thus jointly stabilize the interaction between the U2 snRNP and the tri-snRNP in the human B complex.

\subsection{Aims}

The B-specific proteins interact transiently with the spliceosome at the B complex level. While Prp38, MFAP1 and Snu23 are highly conserved and have been proposed to be essential for the activation of the spliceosome, FBP21, Smu1 and RED are only shared among higher eukaryotes and little is known about their function. Recently, two independent studies came to the conclusion that FBP21 might also help to keep the Brr2 helicase inactive at the critical stage of preparing the spliceosome for its activation (Bertram, Agafonov, Dybkov et al., 2017; Henning et al., 2017). All in all, it appears that the B-specific proteins, conserved or not, are important for supporting or regulating the Brr2-driven rearrangements.

The role of Smu1 and RED, however, remains poorly understood. Although these two proteins have recently been the objects of several studies, each study led to different proposed functions in splicing, so that no clear picture has yet emerged. While Smu1 and RED were first thought to be regulators of alternative splicing (Papasaikas et al., 2015) or mediators of protein-protein interactions (Hegele et al., 2012), more recent considerations rather attribute to them a role in spliceosome stability (Bertram, Agafonov, Dybkov et al., 2017). The one point on which structural and functional studies unanimously agree is that the two proteins undergo a mutual physical interaction that results in a combined, joint function. 


\section{Introduction}

Therefore, my thesis addresses the question of the role that Smu1 and RED actually play during splicing. Currently, it is widely accepted that Smu1 and RED are auxiliary factors that control alternative splicing. However, their transient association with the B complex might indicate an involvement in the formation or stabilization of this assembly intermediate. To reveal whether Smu1 and RED function as regulatory or general splicing factors, I set out to seek convincing evidence by investigating splicing and spliceosome assembly in vivo and in vitro. It has been proposed several times that Smu1 and RED act as a unit during splicing. To understand the importance and nature of this interaction, I further investigated the individual contribution of each protein to their joint function in splicing. A final elucidation of the role of Smu1 and RED within the spliceosome will help us to understand why higher eukaryotes need a more complex spliceosomal protein composition, and how these additional factors enable the spliceosome to fulfil its tasks in more complicated organisms. 


\section{Materials and methods}

\subsection{Materials}

\subsubsection{Chemicals}

\section{Chemical}

Acetic acid

Agarose (low melting point)

Agarose

Ammonium peroxidisulphate (APS)

Ampicillin

Boric acid

Bromphenol blue

Calcium chloride dehydrate $\left(\mathrm{CaCl}_{2} \cdot 2 \mathrm{H}_{2} \mathrm{O}\right)$

Coomassie brilliant blue G-250

Creatine phosphate

Dimethyl pimelimidate (DMP)

Dimethylsulphoxide (DMSO)

Dithiobis succinimidyl propionate (DSP)

Dithiothreitol (DTT)

DNA molecular weight marker (2log, 1 kb)

DNA molecular weight marker (5kb, 100bp)

EDTA (disodium salt dihydrate)

Ethanol

Ethidium bromide

Ficoll 400

Fish sperm DNA $(10 \mathrm{mg} / \mathrm{mL})$

Formamide

Gentamycin

Glycerol

Glycine

GlycoBlue $^{\mathrm{TM}}$ Coprecipitant

Glycogen $(20 \mathrm{mg} / \mathrm{mL})$

Heparin (sodium salt)

HEPES (N-2-Hydroxyethylpiperazin-N-2-ethansulfonic acid)

Hydrochloric acid fuming $37 \%$

Imidazole

\section{Supplier}

Merck, Germany

Thermo Fisher Scientific, USA

Serva, Germany

Serva, Germany

Roth, Germany

Merck, Germany

Serva, Germany

Merck, Germany

Serva, Germany

Sigma-Aldrich, Germany

Thermo Fisher Scientific, USA

Roth, Germany

Thermo Fisher Scientific, USA

Roth, Germany

Roth, Germany

New England Biolabs, Germany

Roth, Germany

Merck, Germany

Serva, Germany

Sigma-Aldrich, Germany

Roche, Germany

Merck, Germany

Roth, Germany

Merck, Germany

Merck, Germany

Thermo Fisher Scientific, USA

Sigma-Aldrich, Germany

Roth, Germany

Sigma-Aldrich, Germany

Merck, Germany

Serva, Germany 


\section{Materials and Methods}

IPTG

Isopropanol

Kanamycin

Maltose

Magnesium chloride hexahydrate $\left(\mathrm{MgCl}_{2} \cdot 6 \mathrm{H}_{2} \mathrm{O}\right)$

Methanol

$\beta$-Mercaptoethanol

Milk powder

MOPS

Nonidet P-40

Penicillin/Streptomycin $(10 \mathrm{mg} / \mathrm{mL})$

PMSF (Phenylmethylsulfonylfluoride)

Poly (ethylene glycol)-block-poly(propylene glycol)block- poly(ethylene glycol) (PEG-PPG) average Mn 14000

Polyvinylpyrrolidone average Mn 10000

Ponceau S

Potassium chloride $(\mathrm{KCl})$

Potassium dihydrogen phosphate $\left(\mathrm{KH}_{2} \mathrm{PO}_{4}\right)$

Potassium hydroxide $(\mathrm{KOH})$

Pre-stained protein-molecular weight marker

Roti phenol-chloroform-isoamyl alcohol (PCl)

Rotiphorese Gel 30 (37.5:1) Polyacrylamide

Rotiphorese Gel 40 (19:1) Polyacrylamide

Sodium acetate

Sodium azide

Sodium chloride $(\mathrm{NaCl})$

Sodium dodecyl sulfate (SDS)

Sodium hydroxide $(\mathrm{NaOH})$

Spermidine

SYBR $^{\circledR}$ Gold Nucleic Acid Gel Stain

TEMED (N, N, N', N'-Tetramethylethylendiamine)

Tetracyclin

Triethanolamine

Tris [Tris-(hydroxymethyl)aminomethane]

Triton X-100

tRNA from $E$. coli

Tween-20

Urea
Roth, Germany

Merck, Germany

Roth, Germany

Merck, Germany

Merck, Germany

Merck, Germany

Roth, Germany

Roth, Germany

Roth, Germany

Thermo Fisher Scientific, USA

Roche, Germany

Sigma-Aldrich, Germany

Sigma-Aldrich, Germany

Serva, Germany

Merck, Germany

Merck, Germany

Merck, Germany

Bio-Rad, Germany

Roth, Germany

Roth, Germany

Roth, Germany

Merck, Germany

Merck, Germany

Merck, Germany

Serva, Germany

Merck, Germany

Sigma-Aldrich, Germany

Thermo Fisher Scientific, USA

Sigma-Aldrich, Germany

Roth, Germany

Sigma-Aldrich, Germany

VWR International, Germany

Sigma-Aldrich, Germany

Roche, Germany

Roth, Germany

Merck, Germany 
X-gal

Xylene cyanol FF

\subsubsection{Consumables}

\section{Consumable}

6-well plates

Biodyne B Membrane

Cell scraper

Centrifugal filters, Amicon Ultra

Centrifuge tubes (TH660)

Centrifuge tubes (TST41.14)

Dialysis membranes (MWCO 6000-8000 Da)

Electroporation cuvettes

NuPAGE ${ }^{\text {TM }}$ gels (1.5 mm, 4-12\%)

Nesco-/Parafilm

Petri dishes (for bacteria)

Greiner CELLSTAR ${ }^{\circledR}$ dish (for adhesive cell lines)

Greiner CELLSTAR ${ }^{\circledR}$ serological pipette

Greiner culture tubes (14 mL)

Protran BA 83 nitrocellulose

ReadyLyzer 3, MWCO 6-8 kDa

Safe-lock tubes $(0.5,1.5$ and $2 \mathrm{~mL})$

Standard Polystyrene Semi-micro Cuvettes

Sterile filters $(0.2 \mu \mathrm{m}$ or $0.45 \mu \mathrm{m})$

Surgical blades

Tips, disposable

Tubes (15 mL and $50 \mathrm{~mL}$ )

Whatman 3MM paper
Sigma-Aldrich, Germany

\section{Supplier}

Greiner-Bio-One, Germany

Pall Corporation, Germany

Sarstedt, Germany

Millipore, USA

Seton scientific, USA

Beranek Laborgeräte, Germany

SpektraPor, USA

Bio-Rad, Germany

Thermo Fisher Scientific, USA

Roth, Germany

Sarstedt, Germany

Greiner, Germany

Greiner, Germany

Greiner, Germany

GE Healthcare, UK

Serva, Germany

Eppendorf, Germany

Sarstedt, Germany

Sarstedt, Germany

Martin, Germany

StarLab, UK

Sarstedt, Germany

Whatman, UK 


\section{Materials and Methods}

\subsubsection{Chromatographic resins and columns}

\section{Resin / column}

Amylose resin

Chromatography columns

Dynabeads $^{\mathrm{TM}}$ Protein A

Roti ${ }^{\circledR}$ garose-His/Ni Beads

ProbeQuant $^{\mathrm{TM}}$ G-50 micro columns

\subsubsection{Commerical kits and buffers}

\section{Kit}

$\mathrm{BCA}^{\mathrm{TM}}$ protein assay kit

ECL Western Blot Detection Kit

High Pure Plasmid Isolation Kit

Lipofectamine RNAiMAX Transfection Reagent

NucleoSpin ${ }^{\circledR}$ Gel and PCR Clean-up

Prime It II random-primer labeling kit

PureLink $^{\mathrm{TM}}$ HiPure Plasmid Filter Maxiprep Kit

X-tremeGENE Tм 9 DNA Transfection Reagent

\section{Buffer}

Gel Loading Dye, Purple (6X), no SDS

Phusion ${ }^{\circledR}$ HF Buffer Pack (5x)

\subsubsection{Machines}

\section{Machine}

Autoclaves

Axiovert 25 Inverted Microscope

Biological safety Cabinets Hera Safe

Biofuge fresco

Biofuge pico

Bioruptor Sonicator

Casy Cell Counter + Analyzer System Model TT

Centrifuge 5424/5424 R

$\mathrm{CO}_{2}$ incubator BBD 6220

\section{Supplier}

New England Biolabs, Germany

Bio-Rad, Germany

Thermo Fisher Scientific, USA

Roth, Germany

GE Healthcare, UK

\section{Supplier}

Thermo Fisher Scientific, USA

GE Healthcare, USA

Roche, Germany

Thermo Fisher Scientific, USA

Macherey-Nagel, Germany

Agilent Technologies, USA

Thermo Fisher Scientific, USA

Sigma-Aldrich, Germany

\section{Supplier}

New England Biolabs, Germany

New England Biolabs, Germany

\section{Supplier}

H+P Labortechnik, Germany

Zeiss, Germany

Thermo Fisher Scientific, USA

Heraeus, Germany

Heraeus, Germany

Diagenode, Belgium

Innovatis, Germany

Eppendorf, Germany

Heraeus, Germany 


\section{Materials and Methods}

EG\&G, and radiation hand-foot monitor

Electroporation system MicroPulser

Fiberlite $^{\text {TM }}$ F14-14 x 50cy Fixed-Angle Rotor

Gel documentation unit

Gel electrophoresis apparatus

Gel electrophoresis apparatus

Gel dryer model 583

Glass cell culture flasks

Glass-ware

Gradient master model 106

Head-over-tail rotor

Heating blocks HB-130

Hybridization oven

Ice machine

IKA-Vibrax-VXR table-top shaker

$\mathrm{KB}$ incubator

LB122, contamination monitor

MaxQ 3000 Benchtop Shaker

Megafuge 1.0R

Milli-Q-water supply apparatus

Mini-Monitor Series 900 Radiation Monitor

Mini Protean 3 Electrophoresis Cell

Mini Trans-Blot Electrophoretic Transfer Cell

Multifuge 1S-R

Multitron incubator shaker

Orion 3 Star $\mathrm{pH}$ meter

Phosphorimager Typhoon 8600

Power supply EPS 2A200

Power supply EPS 3501/XL

Q Exactive ${ }^{\mathrm{TM}} \mathrm{HF}$ Hybrid Quadrupol-Orbitrap ${ }^{\mathrm{TM}}$ Mass

Spectrometer

Rocking Platform WT15

Scintillation counter LS 1701

Sonifier W-250D

Sorvall $^{\mathrm{TM}} \mathrm{LYNX}^{\mathrm{TM}}$ Superspeed Centrifuge

Sorvall TH660 rotor

Sorvall TST41.14 rotor

Sorvall Ultracentrifuge Discovery 90 SE

Sorvall WX Ultracentrifuge
Berthold, Germany

Bio-Rad, Germany

Thermo Fisher Scientific, USA

Bio-Rad, Germany

Bio-Rad, Germany

In-house

Bio-Rad, Germany

Duran Group, Germany

VWR International, Germany

BioComp Instruments, Canada

Cole-Parmer, USA

Unitek, USA

Hybaid Biometra, UK

Ziegra, UK

IKA-Werke, Germany

Binder, Germany

Berthold, Germany

Thermo Fisher Scientific, USA

Kendro, USA

Millipore, USA

Mini Instruments, UK

Bio-Rad, USA

Bio-Rad, USA

Heraeus, Germany

INFORS HT, Swiss

Thermo Fisher Scientific, USA

Amersham Pharmacia, Germany

Hoefer Pharmacia Biotech, USA

Amersham Pharmacia, Germany

Thermo Fisher Scientific, USA

Biometra, Germany

Packard, USA

Heinemann, Germany

Thermo Fisher Scientific, USA

Kendro, USA

Kendro, USA

Sorvall/Kendro, USA

Sorvall/Kendro, USA 


\section{Materials and Methods}

Speed Vac Konzentrator 5301

Spectrophotometer Nanodrop ND-1000

Spectrophotometer Ultrospec 3000 pro

Test tube rotator

T3 Thermocycler

Thermomixer comfort

Trans-Blot Electrophoretic Transfer Cell

UV-Stratalinker 2400

Vortex

X-ray film developer X-Omat 2000
Eppendorf, Germany

Thermo Fisher Scientific, USA

GE Healthcare, USA

Snijders, The Netherlands

Biometra, Germany

Eppendorf, Germany

Bio-Rad, Germany

Stratagene, USA

Scientific industries, USA

Kodak, USA

\section{Supplier}

Pharmacia, Germany

Pharmacia, Germany

Kedar, Poland

\subsubsection{Radiolabeled nucleotides}

\section{Nucleotide}

$\alpha$-32P-UTP [10 $\mu \mathrm{Cl} / \mu \mathrm{l}, 3000 \mathrm{Ci} / \mathrm{mmol}]$

$\alpha-{ }^{32} \mathrm{P}-\mathrm{dATP}[10 \mu \mathrm{Cl} / \mu \mathrm{l}, 3000 \mathrm{Ci} / \mathrm{mmol}]$

\section{Supplier}

Perkin-Elmer, Germany

Perkin-Elmer, Germany

\subsubsection{Oligonucleotides}

$\begin{array}{ll}\text { Name } & \text { Sequence }\left(5^{\prime} \rightarrow 3^{\prime}\right) \\ \text { Smu1-F } & \text { CAATCACTCGACGAAGAC } \\ \text { Smu1-R } & \text { CTTCCGTGTTTCAGTTAGC }\end{array}$




\section{Materials and Methods}

Strep-D-WD40-R

Smu1-His-F

Smu1-His-R

RED-F

RED-R

D-N-RED \#2-F

D-C-RED \#1-R-Stop

Primer 550 (F)

Primer 551 (R)

Primer 552 (F)

Primer $553(\mathrm{R})$

MINX-70 Primer A

MINX-70 Primer B

MINX-70 Primer C

MINX-70 Primer D

MINX-80 Primer A

MINX-80 Primer B

MINX-80 Primer C

MINX-80 Primer D

MINX-90 Primer A

MINX-90 Primer B

MINX-90 Primer C

MINX-90 Primer D

MINX-150 Primer A

MINX-150 Primer $B$

MINX-150 Primer C

MINX-150 Primer D

MINX-80-cleaved Primer A

MINX-80-cleaved Primer B

MINX-80-cleaved Primer C

MINX-80-cleaved Primer D

PM556 and PM5-1056 Primer A

PM5 56 and PM5-1056 Primer B

PM556 and PM5-1056 Primer C

$\mathrm{PM}_{56}$ and PM5-1056 Primer D
ATTATGCATTCATTATTTTTCGAACTGAGGG

TGGGACCACTGTCCGAACTTGATGTGCC

ATACGAATTCCATATGTCTATCGAAATCGAGTCC

ATACCTGCAGGATTTAAGGCTTCCACAGCTTCAGCA

ATGCGAATTCAGCATGGGACCTGAAAGG

ATGCCTGCAGGATGCTCATTAGTACTTAG

ATACGAATTCCATATGAACTACAGGGCCGTCGGACCTACCG

ATACCTGCAGGATCTAGGAGTAGTCCACTTCTTCGTCA

CCATCTCGCAAATAAATAAG

CATTTTATGTTTCAGGTTC

TCATAGCGCGGGTTCCTTCC

CTTTAATTCAACCCAACACA

TCACACAGGAAACAGCTATGAC

TCTTACCGTTCGGAGG

CCTCCGAACGGTAAGAGGGCGCAGTAGTCCAG

GTAAAACGACGGCCAGTG

TCACACAGGAAACAGCTATGAC

AGTTCTACATGCTAGGCTCTTACC

GGTAAGAGCCTAGCATGTAGAACT GTAGTCCAGGGTTTCCTT

GTAAAACGACGGCCAGTG

TCACACAGGAAACAGCTATGAC

AGTTCTACATGCTAGGCTCTTACC

GGTAAGAGCCTAGCATGTAGAACT CTAGGGCGCAGTAGTCCAG

GTAAAACGACGGCCAGTG

TCACACAGGAAACAGCTATGAC

AGACTGAGACTGAGACTGAGGGCTCTTACCGTTCGGAG

CTCAGTCTCAGTCTCAGTCTCAGTCTGCCTgtagaactggttacctgcagcC

GTAAAACGACGGCCAGTG

TCACACAGGAAACAGCTATGAC

TGAGACTGAGACTGAGGGCTC

IAATACGACTCACTATAGGGTCTCAGGGTTTCCTTGATG

GTAAAACGACGGCCAGTG

TCACACAGGAAACAGCTATGAC

GTAAGCTTGATACATACCTTGGC

GCCAAGGTATGTATCAAGCTTACGTGACTGATAGAACACTACCTG

GTAAAACGACGGCCAGTG 


\title{
Materials and Methods
}

$$
\begin{aligned}
& \text { U1 snRNA forward K107 } \\
& \text { U1 snRNA reverse K50 } \\
& \text { U2 snRNA forward K78 } \\
& \text { U2 snRNA reverse K79 } \\
& \text { U4 snRNA forward K47 } \\
& \text { U4 snRNA reverse K48 } \\
& \text { U5 snRNA forward K115 } \\
& \text { U5 snRNA reverse KJ7 } \\
& \text { U6 snRNA forward K54 } \\
& \text { U6 snRNA reverse K55 }
\end{aligned}
$$

\author{
GATACTTACCTGGCAGGGGAG \\ CGCGGATCCAGGGGAAAGCGCGAACGCAGTC \\ CCTAATACTCACTATAGATCGCTTCTCGGCCTTTTGCG \\ GGGTGCACCGTTCCTGGAGGTAC \\ GGGAATTCCTAATACGACTCACTA \\ CGCGAATCCAGTCTCCGTAGAGAC \\ GATACTCTGGTTTCTCTTCAG \\ CCCAAGCTTTAGCCTTGCCAAGGCAAGG \\ CCTAATACGACTCACTATAGGTGCTCGCTTCGGCAGC \\ AAAAATATGGAACGCTTCACG
}

\subsection{9 siRNAs}

$\begin{array}{lll}\text { siRNA } & \text { Target Sequence } & \text { Supplier } \\ \text { Prp38A-1 } & \text { CTGCTGTTATTCAAAGATCAA } & \text { Laboratory of R. Lührmann, MPI-bpc } \\ \text { Prp38A-2 } & \text { TCCACGGACATCGATAATCAA } & \text { Laboratory of R. Lührmann, MPI-bpc } \\ \text { MFAP1-1 } & \text { AAGGACCGAGTGACAGTTCAA } & \text { Laboratory of R. Lührmann, MPI-bpc } \\ \text { MFAP1-2 } & \text { TACGGCGTTTACAGAACCGTA } & \text { Laboratory of R. Lührmann, MPI-bpc } \\ \text { RED-1 } & \text { AACCCGTAACAAGAAGCTTAA } & \text { Laboratory of R. Lührmann, MPI-bpc } \\ \text { RED-2 } & \text { CAGCGAGTATATGAACAACAA } & \text { Laboratory of R. Lührmann, MPI-bpc } \\ \text { Smu1-1 } & \text { CACAACTGAGCAGGCATATTA } & \text { Laboratory of R. Lührmann, MPI-bpc } \\ \text { Smu1-2 } & \text { TACGGCTATGTCGATCGAAAT } & \text { Laboratory of R. Lührmann, MPI-bpc } \\ \text { Smu1-3 } & \text { GTAAAGATCTGGAATATGA } & \text { Laboratory of R. Lührmann, MPI-bpc } \\ \text { AllStars Negative Control siRNA } & \text { QIAGEN }\end{array}$

\subsubsection{Plasmids}

Name
hSmu1_pMK-RQ
RED-IK_pMK-RQ
pFL
pFL-RED-His10
pFL- $\triangle$ N-RED- His10

\section{Description}

Synthetic gene of the full-length Smu1; $\operatorname{Kan}^{\mathrm{R}}$

Synthetic gene of the full-length RED; Kan ${ }^{\mathrm{R}}$

Multibac transfer vector (acceptor) for bacmid preparation; $\mathrm{Gen}^{\mathrm{R}}$ and $\mathrm{Amp}^{\mathrm{R}}$

N-His 10, RED full-length gene inserted via This study

EcoRI/Pstl; Gen ${ }^{R}$ and Amp ${ }^{R}$

$\mathrm{N}-\mathrm{His}_{10}, \mathrm{~N}$-terminally truncated RED gene inserted This study via EcoRI/Pstl; Gen ${ }^{R}$ and $A m p^{R}$

\section{Reference}

Geneart, Germany Geneart, Germany

Dr. Imre Berger, EMBL,

Grenoble

\begin{abstract}
Supplier
\end{abstract}




\section{Materials and Methods}

\begin{tabular}{|c|c|c|}
\hline pFL-RED- $\Delta$ C- His 10 & $\begin{array}{l}\text { N-His10, C-terminally truncated RED gene inserted } \\
\text { via EcoRI/Pstl; Gen }{ }^{R} \text { and } A m p^{R}\end{array}$ & This study \\
\hline $\mathrm{pFL}-\Delta \Delta$-RED- $\mathrm{His}_{10}$ & $\begin{array}{l}\mathrm{N}-\mathrm{His}_{10}, \mathrm{~N} \text { - and } \mathrm{C} \text { - terminally truncated RED gene } \\
\text { inserted via EcoRI/Pstl; Gen }{ }^{R} \text { and } \mathrm{Amp}^{\mathrm{R}}\end{array}$ & This study \\
\hline pFL-Smu1-Strepll & $\begin{array}{l}\text { C-Strepll, Smu1 full-length gene inserted via } \\
\text { Xhol/Nsil; Gen }{ }^{R} \text { and Amp }{ }^{R}\end{array}$ & This study \\
\hline pFL-Smu1- $\Delta$ WD40-Strepll & $\begin{array}{l}\text { C-Strepll, Smu1 WD40 domain deletion mutant } \\
\text { gene inserted via Xhol/Nsil; Gen }{ }^{R} \text { and } A m p^{R}\end{array}$ & This study \\
\hline pFL-Smu1- His 10 & $\begin{array}{l}\text { N-His10, Smu1 full-length gene inserted via } \\
\text { EcoRI/Pstl; Gen }{ }^{R} \text { and } A m p^{R}\end{array}$ & This study \\
\hline pMINX & Plasmid encoding MINX pre-mRNA; Amp ${ }^{R}$ & (Zillmann et al., 1988) \\
\hline pPM5 & Plasmid encoding PM5 pre-mRNA; Amp ${ }^{R}$ & (Bessonov et al., 2008) \\
\hline pPM5-10 & Plasmid encoding PM5-10; Amp ${ }^{R}$ & (Bessonov et al., 2010) \\
\hline
\end{tabular}

\subsubsection{Antibodies}

\section{Antibody}

Anti-hSnu66

Anti-hPrp31, phospho-specific

Anti-P-SF3b155 (T313)

Anti-Prp31

Anti-SF3b155

Anti-RED

Anti-MFAP1

Anti-FBP21

Anti-hSmu-1

Anti-hPrp38

Anti-hSnu23

Anti-H3 (Histone 3)

Anti-Snu114

Anti-SF3A2

Goat-anti-rabbit (horseradish peroxidase coupled)

Goat-anti-mouse (horseradish peroxidase coupled)

\section{Supplier}

Laboratory of R. Lührmann, MPI-bpc (Makarova et al., 2001)

Laboratory of R. Lührmann, MPI-bpc (Schneider, Hsiao et al., 2010)

Laboratory of R. Lührmann, MPI-bpc (Girard et al., 2012) Laboratory of R. Lührmann, MPI-bpc (Makarova et al., 2002)

Laboratory of R. Lührmann, MPI-bpc (Will et al., 2001)

Laboratory of R. Lührmann, MPI-bpc

Laboratory of R. Lührmann, MPI-bpc

Santa Cruz Biotechnology, USA

Santa Cruz Biotechnology, USA

Laboratory of R. Lührmann, MPI-bpc

Laboratory of R. Lührmann, MPI-bpc

Abcam, UK

Laboratory of R. Lührmann, MPI-bpc (Fabrizio et al.,1997, Sidarovich et al., 2017)

Laboratory of R. Lührmann, MPI-bpc (Will et al., 2002)

Jackson Immunoresearch, USA

Jackson Immunoresearch, USA 


\section{Materials and Methods}

\subsubsection{Proteins, enzyme inhibitors and enzymes and}

\section{Protein}

Bovine serum albumin (BSA), acetylated

MS2-MBP

\section{Enzyme inhibitor}

Complete protease inhibitor, EDTA-free

PhosSTOPTM

RNase Inhibitor [40 U/ $\mu \mathrm{l}]$

\section{Enzyme}

Accutase $^{\circledR}$ solution

Pfu Tripple DNA Polymerase

Proteinase K $(10 \mathrm{mg} / \mathrm{mL})$

Restriction endonucleases

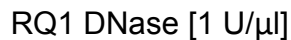

SP6 RNA Polymerase

T4 DNA Ligase

T7 RNA Polymerase [20 U/ $\mu \mathrm{l}]$

Yeast inorganic pyrophosphatase (YIPP) $[0.1 \mathrm{U} / \mu \mathrm{l}]$

\section{Supplier}

Sigma-Aldrich, Germany

Laboratory of R. Lührmann, MPI-bpc

\section{Supplier}

Roche, Germany

Roche, Germany

Molox, Germany

\section{Supplier}

Sigma-Aldrich, Germany

Laboratory of D. Görlich, MPI-bpc

Bioron, Germany

New England Biolabs, Germany

Promega, USA

New England Biolabs, Germany

Laboratory of D. Görlich, MPI-bpc

Laboratory of D. Görlich, MPI-bpc

New England Biolabs, Germany

\subsubsection{Bacteria strains}

\section{Strain}

Escherichia coli XL-10 Gold

Escherichia coli DH10MultiBacY

\section{Supplier}

Agilent Technologies, USA

Dr. Imre Berger, EMBL, Grenoble

\subsubsection{Cell lines}

HeLa S3 cells were used for the preparation of nuclear extract. HeLa SS6 cells were used for RNAi experiments. The Spodoptera frugiperda cell line Sf9 was used for recombinant baculovirus propagation and isolation.

The Trichoplusia ni cell line High Five was used for recombinant protein expression. 


\section{Materials and Methods}

\section{Cell line}

HeLa S3 cells (human cervical cancer cells)

HeLa SS6 cells (human cervical cancer cells)

High Five ${ }^{T M}$ cells

Sf9 cells

\subsubsection{Commercial media}

\section{Medium}

Dulbecco's Modified Eagle Medium (DMEM)

ESF 921 Insect Cell Culture Medium, Protein Free

Gibco $^{\text {TM }}$ Sf-900 ${ }^{\text {TM }}$ III SFM

LB Agar Medium (Powder)

LB Medium (Capsules)

Opti-MEM - Reduced Serum Media

\section{Supplier}

GBF, Germany

ATCC, USA

Invitrogen, Germany

Invitrogen, Germany

\section{Supplier}

Thermo Fisher Scientific, USA

Expression Systems, USA

Fisher Scientific, USA

MP Biomedicals, USA

MP Biomedicals, USA

Thermo Fisher Scientific, USA

\subsubsection{Buffers, media and solutions}

Media, buffers and solutions were prepared with deionized water (Millipore) and autoclaved if necessary $\left(121^{\circ} \mathrm{C}\right.$, $20 \mathrm{~min} / 15 \mathrm{~min} 1$ bar). Solutions with heat-labile components were filter-sterilized $(0.22 \mu \mathrm{m})$.

The $\mathrm{pH}$ was adjusted with $37 \% \mathrm{HCl}, 5 \mathrm{M} \mathrm{NaOH}$ or $5-10 \mathrm{M} \mathrm{KCl}$.

\section{Coomassie staining solution}

$100 \mu \mathrm{M} \quad$ Coomassie brilliant blue G-250

$40 \mathrm{mM} \quad \mathrm{HCl}$

\section{Denhardt's Solution (100x)}

$\begin{array}{ll}2 \% & \text { BSA } \\ 2 \% & \text { SDS } \\ 2 \% & \text { Polyvinylpyrrolidone }\end{array}$

\section{0x G-75 buffer}

$200 \mathrm{mM} \quad$ HEPES

$750 \mathrm{mM} \quad \mathrm{KCl}$

$15 \mathrm{mM} \quad \mathrm{MgCl}_{2}$

$\mathrm{pH}$ adjusted to 7.9 


\section{Materials and Methods}

\section{0x G-150 buffer}

$200 \mathrm{mM}$

HEPES

$1.5 \mathrm{M}$

$\mathrm{KCl}$

$15 \mathrm{mM}$

$\mathrm{MgCl}_{2}$

$\mathrm{pH}$ adjusted to 7.9

\section{Gel fixing solution}

$\begin{array}{ll}10 \%(\mathrm{v} / \mathrm{v}) & \text { Acetic acid } \\ 40 \% & \text { Ethanol or Methanol }\end{array}$

\section{HeLa Cell Lysis Buffer}

$\begin{array}{ll}2 \mathrm{mM} & \text { EDTA, pH } 8.0 \\ 150 \mathrm{mM} & \mathrm{NaCl} \\ 30 \mathrm{mM} & \text { Tris- } \mathrm{HCl}, \mathrm{pH} 7.5 \\ 1 \% & \text { Triton } \mathrm{X}-100 \\ 2 \mathrm{x} & \text { PhosSTOP }{ }^{\mathrm{TM}} \\ 2 \mathrm{x} & \text { Complete protease inhibitor } \\ 1 \mathrm{mM} & \mathrm{MgCl}_{2}\end{array}$

\section{HeLa IP Dilution Bufer}

\begin{tabular}{|c|c|}
\hline $235 \mathrm{mM}$ & $\mathrm{NaCl}$ \\
\hline $30 \mathrm{mM}$ & Tris- $\mathrm{HCl}, \mathrm{pH} 7.5$ \\
\hline $2 \%$ & Triton $\mathrm{X}-100$ \\
\hline $2 x$ & PhosSTOP тм \\
\hline $2 x$ & Complete protease inhibitor \\
\hline $3 \mathrm{mM}$ & $\mathrm{MgCl}_{2}$ \\
\hline $0.2 \mathrm{U} / \mu \mathrm{L}$ & RNasin \\
\hline
\end{tabular}

\section{HeLa IP Wash Bufer}

$\begin{array}{ll}200 \mathrm{mM} & \mathrm{NaCl} \\ 30 \mathrm{mM} & \text { Tris- } \mathrm{HCl}, \mathrm{pH} 7.5 \\ 1 \% & \text { Triton } \mathrm{X}-100 \\ 1.5 \mathrm{mM} & \mathrm{MgCl}_{2}\end{array}$




\section{HeLa Sonication Buffer}

$\begin{array}{ll}2 \mathrm{mM} & \text { EDTA, pH } 8.0 \\ 60 \mathrm{mM} & \text { Tris-HCl, pH } 7.5 \\ 2 \% & \text { PEG-PPG } \\ 2 \mathrm{x} & \text { PhosSTOP }{ }^{\mathrm{TM}} \\ 2 \mathrm{x} & \text { Complete protease inhibitor }\end{array}$

\section{HeLa IP Dilution Bufer}

$\begin{array}{ll}2 \mathrm{mM} & \text { EDTA, } \mathrm{pH} 8.0 \\ 30 \mathrm{mM} & \text { Tris-HCl, pH } 7.5 \\ 1 \% & \text { Triton } \mathrm{X}-100 \\ 2 \mathrm{x} & \text { PhosSTOP }{ }^{\mathrm{TM}} \\ 2 \mathrm{x} & \text { Complete protease inhibitor }\end{array}$

$0.3 \mathrm{U} / \mathrm{\mu L} \quad$ RNasin

\section{High Five Lysis Buffer}

$\begin{array}{ll}50 \mathrm{mM} & \text { HEPES-KOH, pH } 7.9 \\ 500 \mathrm{mM} & \mathrm{NaCl} \\ 15 \mathrm{mM} & \text { Imidazole, } \mathrm{pH} 8.0 \\ 0.1 \% & \text { NP-40 } \\ 15 \% & \text { Glycerol } \\ 2 \mathrm{mM} & \text { DTT }\end{array}$

\section{High Five Dilution Buffer}

$\begin{array}{ll}50 \mathrm{mM} & \text { HEPES-KOH, pH } 7.9 \\ 50 \mathrm{mM} & \mathrm{NaCl} \\ 15 \% & \text { Glycerol } \\ 2 \mathrm{mM} & \text { DTT }\end{array}$

\section{High Five Wash Buffer}

$\begin{array}{ll}50 \mathrm{mM} & \text { HEPES-KOH, pH } 7.9 \\ 750 \mathrm{mM} & \mathrm{NaCl} \\ 20 \mathrm{mM} & \text { Imidazole } \\ 15 \% & \text { Glycerol } \\ 2 \mathrm{mM} & \text { DTT }\end{array}$




\section{Materials and Methods}

\section{High Five Elution Buffer}

$\begin{array}{ll}20 \mathrm{mM} & \text { HEPES-KOH, pH } 7.9 \\ 200 \mathrm{mM} & \mathrm{NaCl} \\ 300 \mathrm{mM} & \text { Imidazole } \\ 15 \% & \text { Glycerol } \\ 2 \mathrm{mM} & \text { DTT }\end{array}$

20x MOPS buffer

$\begin{array}{ll}1 \mathrm{M} & \text { Tris } \\ 1 \mathrm{M} & \text { MOPS } \\ 2 \% & \text { SDS }\end{array}$

$20 \mathrm{mM} \quad$ EDTA

\section{$\underline{5 x \text { Native gel loading dye }}$}

$\begin{array}{ll}90 \mathrm{mM} & \text { Tris } \\ 90 \mathrm{mM} & \text { Boric acid } \\ 2.5 \mathrm{mM} & \text { EDTA, pH } 8.0 \\ 30 \%(\mathrm{v} / \mathrm{v}) & \text { Glycerol } \\ 0.05 \%(\mathrm{w} / \mathrm{v}) & \text { Bromphenol blue }\end{array}$

Northern blot transfer buffer

$\begin{array}{ll}176 \mathrm{mM} & \mathrm{NaH}_{2} \mathrm{PO}_{4} \\ 90 \mathrm{mM} & \mathrm{Na}_{2} \mathrm{HPO}_{4}\end{array}$

Northern blot hybridization solution

$\begin{array}{ll}50 \%(\mathrm{v} / \mathrm{v}) & \text { Formamide } \\ 10 \mathrm{x} & \text { SSC } \\ 0.5 \% & \text { SDS } \\ 30 \mathrm{mM} & \text { Tris-HCl, pH 7.5 } \\ 5 \mathrm{x} & \text { Denhardt's Solution }\end{array}$

\section{Northern blot wash buffer}

$2 \mathrm{x} \quad \mathrm{SSC}$

$0.1 \% \quad$ SDS 


\section{0x PBS (-T)}

$\begin{array}{ll}1.3 \mathrm{M} & \mathrm{NaCl} \\ 27 \mathrm{mM} & \mathrm{KCl} \\ 80 \mathrm{mM} & \mathrm{Na}_{2} \mathrm{HPO}_{4} \\ 15 \mathrm{mM} & \mathrm{KH}_{2} \mathrm{PO}_{4} \\ 0.05 \% & \text { Tween-20 (optional) }\end{array}$

\section{PK Buffer}

$\begin{array}{ll}50 \mathrm{mM} & \text { Tris- } \mathrm{HCl}, \mathrm{pH} 7.5 \\ 70 \mathrm{mM} & \mathrm{NaCl} \\ 0.2 \% & \mathrm{SDS} \\ 1 \mathrm{mM} & \mathrm{CaCl}_{2} \\ 0.1 \mathrm{U} / \mu \mathrm{L} & \text { RNase inhibitor }\end{array}$

\section{2x RNA loading dye}

$\begin{array}{ll}80 \% & \text { Formamide } \\ 1 \mathrm{mM} & \text { EDTA, pH } 8.0 \\ 0.05 \%(\mathrm{w} / \mathrm{v}) & \text { Bromphenol blue } \\ 0.05 \%(\mathrm{w} / \mathrm{v}) & \text { Xylene cyanol }\end{array}$

\section{Roeder D buffer}

$\begin{array}{ll}10 \%(\mathrm{v} / \mathrm{v}) & \text { Glycerol } \\ 20 \mathrm{mM} & \text { HEPES-KOH, pH } 7.9 \\ 100 \mathrm{mM} & \mathrm{KCl} \\ 1.5 \mathrm{mM} & \mathrm{MgCl}_{2} \\ 0.2 \mathrm{mM} & \text { EDTA, pH } 8.0 \\ 0.25 \mathrm{mM} & \text { DTT } \\ 0.25 \mathrm{mM} & \text { PMSF }\end{array}$

\section{6x SDS Loading Dye}

$\begin{array}{ll}244 \mathrm{mM} & \text { Tris-HCl, } \mathrm{pH} 7.5 \\ 2.4 \% & \text { SDS } \\ 250 \mathrm{mM} & \text { DTT } \\ 21.2 \%(\mathrm{w} / \mathrm{v}) & \text { Glycerol } \\ 0.12 \%(\mathrm{w} / \mathrm{v}) & \text { Bromphenol blue }\end{array}$




\section{Materials and Methods}

\section{SDS-PAGE running buffer}

$\begin{array}{ll}25 \mathrm{mM} & \text { Tris- } \mathrm{HCl}, \mathrm{pH} 6.8 \\ 192 \mathrm{mM} & \text { Glycine } \\ 1 \%(\mathrm{w} / \mathrm{v}) & \text { SDS } \\ 21.2 \%(\mathrm{w} / \mathrm{v}) & \text { Glycerol } \\ 0.12 \%(\mathrm{w} / \mathrm{v}) & \text { Bromphenol blue }\end{array}$

\section{0x SSC buffer}

$3 \mathrm{M} \quad \mathrm{NaCl}$

$300 \mathrm{mM} \quad$ Trisodium citrate

$\mathrm{pH}$ adjusted to 7.9

\section{0x T4 DNA ligase buffer}

$500 \mathrm{mM} \quad$ Tris- $\mathrm{HCl}, \mathrm{pH} 7.5$

$100 \mathrm{mM} \quad \mathrm{MgCl}_{2}$

$100 \mathrm{mM} \quad$ DTT

$10 \mathrm{mM} \quad$ ATP

$250 \mu \mathrm{g} / \mathrm{mL} \quad$ BSA

\section{0x TBE}

$0.89 \mathrm{M} \quad$ Tris- $\mathrm{HCl}, \mathrm{pH} 7.5$

$0.89 \mathrm{M} \quad$ Boric acid

$25 \mathrm{mM} \quad$ EDTA, $\mathrm{pH} 8.0$

\section{0x TBS-T}

$200 \mathrm{mM} \quad$ Tris- $\mathrm{HCl}, \mathrm{pH} 7.5$

$1.5 \mathrm{M} \quad \mathrm{NaCl}$

$1 \% \quad$ Tween-20

\section{Triethanolamine solution}

1x PBS

$200 \mathrm{mM} \quad$ Triethanolamine

$\mathrm{pH}$ adjusted to 8.6 


\section{5x TRO buffer}

$\begin{array}{ll}1 \mathrm{M} & \text { HEPES-KOH, } \mathrm{pH} 7.5 \\ 200 \mathrm{mM} & \text { DTT } \\ 160 \mathrm{mM} & \mathrm{MgCl}_{2} \\ 10 \mathrm{mM} & \text { Spermidine }\end{array}$

\section{Western blot blocking buffer}

$\begin{array}{ll}1 \mathrm{x} & \text { TBS-T } \\ 5 \%(\mathrm{w} / \mathrm{v}) & \text { Milk powder }\end{array}$

\section{Western blot stripping solution}

$\begin{array}{ll}62.5 \mathrm{mM} & \text { Tris-HCl, } \mathrm{pH} 6.7 \\ 2 \%(\mathrm{w} / \mathrm{v}) & \mathrm{SDS} \\ 85 \mathrm{mM} & \beta \text {-Mercaptoethanol }\end{array}$

\section{0x Western blot transfer buffer}

$\begin{array}{ll}500 \mathrm{mM} & \text { Tris- } \mathrm{HCl}, \mathrm{pH} 7.5 \\ 400 \mathrm{mM} & \text { Glycine } \\ 0.1 \%(\mathrm{w} / \mathrm{v}) & \text { SDS }\end{array}$

\subsection{Methods}

\subsubsection{Molecular biology standard methods}

2.2.1.1 PCR amplification

DNA fragments were amplified in a volume of $50 \mu \mathrm{L}$ by PCR as illustrated in Tables 2.1 and 2.2.

\begin{tabular}{lrr}
$\begin{array}{l}\text { Table 2.1: Composition of a standard PCR } \\
\text { Component }\end{array}$ & \\
\cline { 2 - 3 } Stock conc. & Final conc. \\
HF Buffer & $5 \mathrm{x}$ & $1 \mathrm{x}$ \\
dNTP mix & $10 \mathrm{mM}$ & $200 \mu \mathrm{M}$ \\
Primer 1 (Fw) & $10 \mu \mathrm{M}$ & $200 \mathrm{nM}$ \\
Primer 2 (Rev) & $10 \mu \mathrm{M}$ & $200 \mathrm{nM}$ \\
Template (plasmid) & & $1 \mathrm{ng} / \mu \mathrm{L}$ \\
Pfu Tripple DNA Pol. & $100 \mathrm{ng} / \mu \mathrm{L}$ & $1 \mathrm{ng} / \mu \mathrm{L}$
\end{tabular}




\section{Materials and Methods}

Table 2.2: Program of a standard PCR

$\left.\begin{array}{lrr}\text { Step } & \text { Temperature } & \text { Time } \\ \text { Initial denaturation } & 95^{\circ} \mathrm{C} & 3 \mathrm{~min} \\ \text { Denaturation } & 95^{\circ} \mathrm{C} & 45 \mathrm{sec} \\ \text { Annealing } & \text { Primer specific } & 40 \mathrm{sec} \\ \text { Extension } & 72^{\circ} \mathrm{C} & 1-2 \mathrm{~min} / \mathrm{kb}\end{array}\right] 31$ cycles

\subsubsection{Restriction digest of DNA}

Restriction digests of PCR products and plasmids were performed in a reaction volume of $50 \mu \mathrm{L}$ at $37^{\circ} \mathrm{C}$ overnight (Table 2.3). When possible, PCR products were digested directly in PCR reaction buffer. For digestions with enzymes incompatible with this buffer, PCR products were purified by using the NucleoSpin ${ }^{\circledR}$ Gel and PCR Cleanup kit from Macherey-Nagel before digestion.

Table 2.3: Composition of a standard restriction digest

$\begin{array}{lrr}\text { Component } & \text { Stock conc. } & \text { Final conc. } \\ \text { Cut Smart Buffer } & 10 \mathrm{x} & 1 \mathrm{x} \\ \text { Enzyme 1 } & 10 \mathrm{U} / \mu \mathrm{g} \text { DNA } \\ \text { Enzyme 2 } & 10 \mathrm{U} / \mu \mathrm{g} \text { DNA } \\ \text { DNA } & 1-5 \mu \mathrm{g}\end{array}$

Digested PCR products were purified directly by using the NucleoSpin ${ }^{\circledR}$ Gel and PCR Clean-up kit, while plasmids were resolved on a preparative agarose gel and the band of interest was used for gel extraction with the NucleoSpin ${ }^{\circledR}$ Gel and PCR Clean-up kit.

\subsubsection{Ligation of digested insert and vector}

The composition of a standard ligation reaction is illustrated in Table 2.4. The ligation was performed in $20 \mu \mathrm{L}$ reaction volume at $16{ }^{\circ} \mathrm{C}$ overnight and immediately transformed into chemically competent $E$. coli cells.

Table 2.4: Composition of a standard ligation reaction

\section{Component}

T4 DNA ligase buffer

T4 DNA ligase

Vector

Insert

\section{Stock conc. Final conc.}

$\begin{array}{rr}10 \mathrm{x} & 1 \mathrm{x} \\ 1 \mathrm{U} / \mu \mathrm{L} & 0.1 \mathrm{U} / \mu \mathrm{L} \\ & 50-100 \mathrm{ng} \\ & 300 \mathrm{ng}-1.5 \mu g\end{array}$

$300 \mathrm{ng}-1.5 \mu \mathrm{g}$ 


\title{
Materials and Methods
}

\subsubsection{Nucleic acid quantification}

To determine the nucleic acid concentration of an aqueous solution, its absorption at wavelengths of $260 \mathrm{~nm}$ and $280 \mathrm{~nm}$ was measured by using a NanoDrop spectrophotometer according to the manufacturer's instructions. The respective buffer without any nucleic acids served as reference for the measurement.

The ratio of OD260/OD280 indicates the purity of the solution. Thereby, a ratio of 2.0 is considered to correspond to pure RNA or oligonucleotides and 1.8 to pure DNA. Ratios below 1.8 indicate the presence of impurities like protein, phenol or other contaminants absorbing at $280 \mathrm{~nm}$.

The following equations were used to determine the concentrations:

\author{
1 OD260: $50 \mu \mathrm{g} / \mathrm{ml}$ double-stranded DNA \\ 1 OD260: $33 \mu \mathrm{g} / \mathrm{ml}$ single-stranded DNA \\ 1 OD260: $40 \mu \mathrm{g} / \mathrm{ml}$ single-stranded RNA
}

\subsubsection{PCl extraction}

To separate nucleic acids from proteins, a phenol-chloroform-isoamyl alcohol (PCI) extraction was performed. During this procedure, the proteins are denatured by the phenol/chloroform mixture and retained in the organic phase, whereas the nucleic acids remain in the aqueous phase. The reactions' volume was adjusted to $200 \mu \mathrm{l}$ and an isovolume of $\mathrm{PCl}$ was added; the samples were thoroughly mixed on a table-top shaker for $10 \mathrm{~min}$. The suspension was centrifuged for $10 \mathrm{~min}$ at room temperature at $13000 \mathrm{rpm}$ in a microfuge to separate the aqueous from the organic phase. The aqueous phase was collected and supplemented with 0.1 volumes $3 \mathrm{M} \mathrm{NaOAC} \mathrm{pH} 5$, 3 volumes absolute ethanol and if required $1 \mu \mathrm{L}$ of GlycoBlue ${ }^{\mathrm{TM}}$ Coprecipitant. Precipitation was performed at -20 ${ }^{\circ} \mathrm{C}$ for at least an hour, usually overnight. The nucleic acids were pelleted by centrifugation at $13000 \mathrm{rpm}$ and $4{ }^{\circ} \mathrm{C}$ for $45 \mathrm{~min}$ in a microfuge and the pellet was washed once with $75 \%(\mathrm{v} / \mathrm{v})$ ethanol. The pellet was then air-dried and resuspended in water or 1x RNA Loading dye.

\subsubsection{Proteinase K digestion}

To prune RNAs of associated proteins, a proteinase $\mathrm{K}$ digest was performed before the $\mathrm{PCl}$ extraction. $\mathrm{PK}$ buffer and $0.5 \mu \mathrm{g} / \mu \mathrm{l}$ proteinase $\mathrm{K}$ was added to the RNA sample and incubated at $37^{\circ} \mathrm{C}$ for $30 \mathrm{~min}$. The RNA was subsequently isolated by $\mathrm{PCl}$ extraction as described in (2.2.1.5). 


\section{Materials and Methods}

\subsubsection{Generation of truncated MINX, PM5 and PM5-10 constructs}

The truncated MINX constructs MINX-70, -80 and -90 were generated by fusion PCR. The MINX pre-mRNA, cloned in PUC18 plasmid, served as template. In brief, a set of four primers was designed. Two primers were placed upstream and downstream of the MINX construct (Fig. 2.1 primers $A$ and D), while the two other primers flanked the sequence to be deleted (primers $B$ and $C$ ). In the first step, the fragments upstream (fragment 1) and downstream (fragment 2) of the region to be deleted were amplified separately. The 5 ' end of the forward primer (primer C), used to amplify the fragment 2 was equipped with a 20 -nucleotide stretch complementary to the 3' extremity of the fragment 1 . The gel-purified fragments served as template in a second PCR reaction. Here, the two fragments were first annealed, and this was followed by synthesis of the sequences complementary to the 5' overhangs by the Pfu DNA polymerase. The resulting constructs, containing the deletion, were subsequently amplified in a third PCR using the primers upstream and downstream of the MINX sequence (primers $A$ and D). The final PCR product was gel-purified and used as template for in vitro transcription. PM556 and PM5-1056 were generated by following a similar strategy.

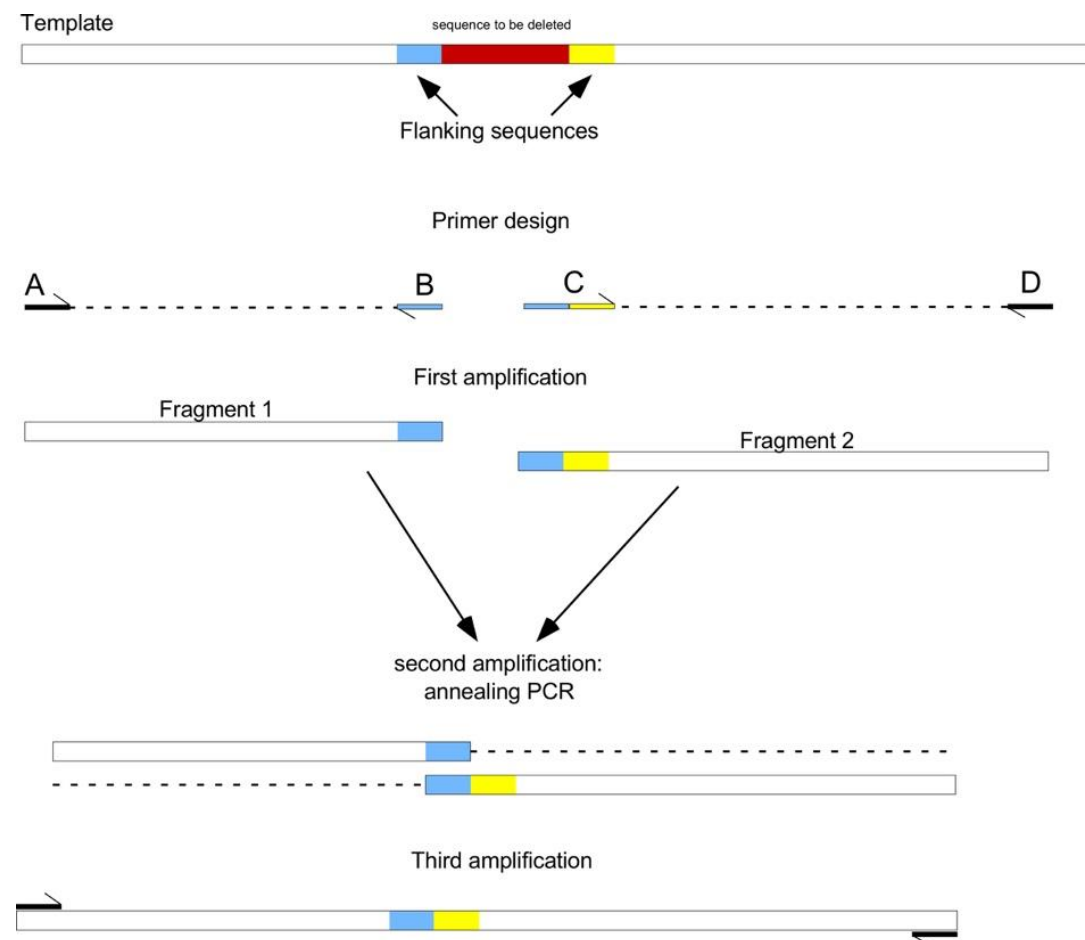

Figure 2.1. Schematic illustration of a fusion PCR. A fusion PCR consists of three PCR reactions. In the first reaction, the two fragments of interest are individually amplified using primer $\mathrm{A}$ and $\mathrm{B}$ or $\mathrm{C}$ and $\mathrm{D}$, respectively. Primer $\mathrm{C}$ includes an overhang complementary to the 3' sequence of Fragment 1, allowing the annealing of the two fragments in a second PCR. The hybridized region serves as a priming sequence for the DNA polymerase, which synthesizes the missing strands. In a third PCR the final product is amplified to obtain sufficient quantities. 
The same principle has also been applied to generate the MINX-150 construct. Here, the primers B and C were positioned next to one another flanking the zone where the additional sequence was to be inserted. The primer $B$ and primer $\mathrm{C}$ contained overhangs with the sequence to be inserted, which in part overlapped.

Table 2.5: Composition of reaction mixtures for the first and third amplifications

\begin{tabular}{lrr} 
Component & Stock conc. & Final conc. \\
\cline { 2 - 3 } HF Buffer & $5 \mathrm{x}$ & $1 \mathrm{x}$ \\
dNTP mix & $10 \mathrm{mM}$ & $200 \mu \mathrm{M}$ \\
Primer 1 (Fw) & $10 \mu \mathrm{M}$ & $200 \mathrm{nM}$ \\
Primer 2 (Rev) & $10 \mu \mathrm{M}$ & $200 \mathrm{nM}$ \\
Template & & \\
(plasmid)/ & & $0.4 \mathrm{ng} / \mu \mathrm{L}$ \\
(PCR product) & & $0.04 \mathrm{ng} / \mu \mathrm{L}$ \\
Pfu Tripple DNA Pol. & $100 \mathrm{ng} / \mu \mathrm{L}$ & $1 \mathrm{ng} / \mu \mathrm{L}$
\end{tabular}

Table 2.6: Program of the first and third amplifications

$\left.\begin{array}{lrr}\text { Step } & \text { Temperature } & \text { Time } \\ \text { Initial denaturation } & 95^{\circ} \mathrm{C} & 3 \mathrm{~min} \\ \text { Denaturation } & 95^{\circ} \mathrm{C} & 30 \mathrm{sec} \\ \text { Annealing } & 56-62^{\circ} \mathrm{C} & 30 \mathrm{sec} \\ \text { Extension } & 72^{\circ} \mathrm{C} & 1 \mathrm{~min} \\ \text { Final extension } & 72^{\circ} \mathrm{C} & 5 \mathrm{~min}\end{array}\right\} 29 \mathrm{CyCles}$

Table 2.7: Composition of reaction mixtures for the second amplification (annealing)

\begin{tabular}{lrr} 
Component & Stock conc. & Final conc. \\
\cline { 2 - 3 } HF Buffer & $5 \mathrm{x}$ & $1 \mathrm{x}$ \\
dNTP mix & $10 \mathrm{mM}$ & $200 \mu \mathrm{M}$ \\
Primer 1 (Fw) & $10 \mu \mathrm{M}$ & $200 \mathrm{nM}$ \\
Primer 2 (Rev) & $10 \mu \mathrm{M}$ & $200 \mathrm{nM}$ \\
Templates & & \\
(Fragment 1) & & \\
(Fragment 2) & & $0.4 \mathrm{ng} / \mu \mathrm{L}$ \\
Pfu Tripple DNA Pol. & $100 \mathrm{ng} / \mu \mathrm{L}$ & $1 \mathrm{ng} / \mu \mathrm{L}$
\end{tabular}

Table 2.8: Program of the second amplification (annealing)

$\begin{array}{lrr}\text { Step } & \text { Temperature } & \text { Time } \\$\cline { 2 - 3 } \text {$\left.Initial denaturation } & 95^{\circ} \mathrm{C} & 3 \mathrm{~min} \\ \text { Denaturation } & 95^{\circ} \mathrm{C} & 30 \mathrm{sec} \\ \text { Annealing } & 55^{\circ} \mathrm{C} & 45 \mathrm{sec} \\ \text { Extension } & 72^{\circ} \mathrm{C} & 1 \mathrm{~min} \\ \text { Final extension } & 72^{\circ} \mathrm{C} & 5 \mathrm{~min}\end{array}\right\}$




\section{Materials and Methods}

\subsubsection{Generation of MINX-80-cleaved}

The MINX-80-cleaved construct was generated by PCR as two separate fragments.

Amplification of the 5' fragment (MINX-80-cleaved Primer A and B) comprised exon 1 and 25 nucleotides downstream of the 5'SS.

The 3' fragment (MINX-80-cleaved Primer C and D) comprised the remaining intronic sequence including the branch site and polypyrimidine tract as well as the exon 2.

To allow in vitro transcription Primer C contained the T7 promoter sequence at its 5' extremity (underlined).

Both fragments were gel purified and used as template in in vitro transcription reactions.

\subsubsection{In vitro transcription}

For in vitro transcription of radioactively labelled or non-labelled pre-mRNAs, linearized plasmids or PCR products were used as DNA templates (see Section 2.2.1.7 and 2.2.1.8). To improve the transcript's stability and to allow affinity purification, all pre-mRNAs were equipped with a $\mathrm{m}^{7} \mathrm{G}\left(5^{\prime}\right) \mathrm{ppp}\left(5^{\prime}\right) \mathrm{G}$-cap and a MS2-tag, respectively. Only part B of trans-MINX-80 was transcribed without cap. MINX pre-mRNA constructs were synthesized by the T7 RNA polymerase and PM5 or PM5-10 constructs by the SP6 RNA polymerase.

Table 2.9 illustrates the composition of a standard in vitro transcription reaction. For transcriptions of trans-MINX80 part $B$, the $\mathrm{m}^{7} \mathrm{G}\left(5^{\prime}\right) \operatorname{ppp}\left(5^{\prime}\right) \mathrm{G}$-cap was excluded and the final concentration of GTP was raised to $7.5 \mathrm{mM}$. For transcription of non-radioactive pre-mRNAs $\alpha-{ }^{32}$ P-UTP was omitted and the final concentration of UTP increased to $7.5 \mathrm{mM}$.

Table 2.9: Composition of a standard in vitro transcription reaction

$\begin{array}{lrrrr}\text { Component } & \text { Stock conc. } & \text { Radioactive } & \text { Without cap } & \text { Non-radioactive } \\ \text { TRO buffer } & 5 \mathrm{x} & 1 \mathrm{x} & 1 \mathrm{x} & 1 \mathrm{x} \\ \text { ATP } & 100 \mathrm{mM} & 7.5 \mathrm{mM} & 7.5 \mathrm{mM} & 7.5 \mathrm{mM} \\ \text { CTP } & 100 \mathrm{mM} & 7.5 \mathrm{mM} & 7.5 \mathrm{mM} & 7.5 \mathrm{mM} \\ \text { UTP } & 100 \mathrm{mM} & 1.5 \mathrm{mM} & 1.5 \mathrm{mM} & 7.5 \mathrm{mM} \\ \text { GTP } & 100 \mathrm{mM} & 1.5 \mathrm{mM} & 7.5 \mathrm{mM} & 1.5 \mathrm{mM} \\ \text { m}^{7} \text { Gcap } & 114 \mathrm{mM} & 5 \mathrm{mM} & & 5 \mathrm{mM} \\ \text { Rnasin } & 40 \mathrm{U} / \mu \mathrm{L} & 1.5 \mathrm{U} / \mu \mathrm{L} & 1.5 \mathrm{U} / \mu \mathrm{L} & 1.5 \mathrm{U} / \mu \mathrm{L} \\ \text { YIPP } & 100 \mathrm{U} / \mu \mathrm{L} & 1.25 \mathrm{U} / \mu \mathrm{L} & 1.25 \mathrm{U} / \mu \mathrm{L} & 1.25 \mathrm{U} / \mu \mathrm{L} \\ \text { T7/SP6 RNA Pol. } & 20 \mathrm{U} / \mu \mathrm{L} & 20 \mathrm{U} / \mu \mathrm{L} & 20 \mathrm{U} / \mu \mathrm{L} & 20 \mathrm{U} / \mu \mathrm{L} \\ & & 10 \mathrm{ng} / \mu \mathrm{L} \mathrm{PCR} & 10 \mathrm{ng} / \mu \mathrm{L} \mathrm{PCR} & 10 \mathrm{ng} / \mu \mathrm{L} \mathrm{PCR} \\ \text { Template } & & 50 \mathrm{ng} / \mu \mathrm{L} \mathrm{plasmid} & 50 \mathrm{ng} / \mu \mathrm{L} \mathrm{plasmid} & 50 \mathrm{ng} \mid \mu \mathrm{L} \mathrm{plasmid} \\ \alpha-{ }^{32} \mathrm{P}-U T P & 750 \mathrm{Ci} / \mathrm{mmol} & 750 \mathrm{Ci} / \mathrm{mmol} & - \\ & 3000 \mathrm{Ci} / \mathrm{mmol} & & \end{array}$




\section{Materials and Methods}

The transcription was performed at $37^{\circ} \mathrm{C}$ for $3 \mathrm{~h}$. The DNA template was digested by addition of $0.5 \mu \mathrm{RQ} 1 \mathrm{DNase}$ $(1 \mathrm{U} / \mu \mathrm{l}, \mathrm{NEB})$ followed by incubation at $37^{\circ} \mathrm{C}$ for $30 \mathrm{~min}$. To eliminate unincorporated nucleotides and proteins, the transcription reaction was purified on a ProbeQuant G-50 column (GE Healthcare) according to the manufacturer's protocol and subsequently subjected to $\mathrm{PCl}$ extraction (Section 2.2.1.5).

\subsubsection{Denaturing polyacrylamide gel electrophoresis}

Pre-mRNA splicing reactions or snRNA samples were resolved by denaturing polyacrylamide gel electrophoresis (PAGE) in the presence of $8 \mathrm{M}$ urea. Denaturing gels were composed of $1 \mathrm{x}$ TBE, $8 \mathrm{M}$ urea and 6-14\% (v/v) polyacrylamide $(40 \%, 19: 1)$, depending on the size of the RNAs to be analysed. Polymerization was initiated by addition of $0.06 \%$ (w/w) APS and $0.06 \%$ TEMED. RNA samples were dissolved in RNA loading dye, denatured for $10 \mathrm{~min}$ at $80^{\circ} \mathrm{C}$ and subsequently chilled on ice. The electrophoresis was performed in 1x TBE buffer at 20-35 mA. The RNA was visualized by autoradiography, with the Phosphorimager Typhoon 8600 or with SYBR ${ }^{\circledR}$ Gold Nucleic Acid Gel Stain.

\subsubsection{SYBR ${ }^{\circledR}$ Gold Nucleic Acid Gel Staining}

To stain snRNA resolved by denaturing PAGE (Section 2.2.1.10), gels were fixed in RNA gel-fixing solution for 30 min at room temperature. Next, the gels were soaked in water for 3 times 10 min each and equilibrated in $0.5 x$ TBE buffer for 2 times $10 \mathrm{~min}$. Staining was performed with 1x SYBR ${ }^{\circledR}$ Gold Nucleic Acid Gel Stain in $0.5 x$ TBE for 15 min. Since the stain is light-sensitive, the gels were protected from light. After a short rinse with $0.5 x$ TBE buffer, RNAs were visualized using the Fluorescence function of the Phosphorimager Typhoon 8600.

\subsubsection{Agarose gel electrophoresis of nucleic acids}

Analysis or purification of DNA samples, such as PCR products or digested plasmids, was performed by agarosegel electrophoresis: Agarose gels contained $1 \mathrm{x}$ TBE and $0.8-2 \%$ agarose depending on the size of the DNA fragments. For DNA visualization gels were stained with ethidium bromide, an organic intercalating agent, at a concentration of $0.5 \mu \mathrm{g} / \mathrm{ml}$. Samples were mixed with Purple Gel Loading Dye (6x, NEB) and the DNA content was separated by electrophoresis at 60-120 V in 1x TBE buffer. In order to evaluate the size of the DNA fragments a commercial DNA ladder (Roth, NEB) was loaded next to the samples. To recover specific DNA fragments from the gel, the DNA was visualized with UV light at a wavelength of $365 \mathrm{~nm}$ and the band of interest was excised with a sterile razor blade. 


\section{Materials and Methods}

The DNA was extracted from the gel by using the NucleoSpin ${ }^{\circledR}$ Gel and PCR Clean-up (Macherey-Nagel) as described in the user's manual. This includes melting of the gel piece, binding of the DNA to the provided matrix and the subsequent elution of the purified fragment. The concentration of the eluted DNA was determined by Nanodrop (as described in Section 2.2.1.4).

\subsubsection{Protein-biochemistry standard methods}

\subsubsection{Protein quantification}

To measure the concentration of protein samples, the $\mathrm{BCA}^{\mathrm{TM}}$ protein assay kit (Thermo Fisher Scientific) was used according to the manufacturer's protocol. This assay is based on bicinchoninic acid (BCA) for the colorimetric detection. A BSA standard was prepared for calibration and the quantification of total protein was analysed at an absorption maximum of $562 \mathrm{~nm}$. Since the assay only tolerates an imidazole concentration up to $50 \mathrm{mM}$, samples with higher concentrations (proteins purified from insect cells) could not be quantified by this method. These were estimated by quantification of gel bands, which were related to protein bands of similar size with known concentration.

\subsubsection{Denaturing SDS polyacrylamide gel-electrophoresis (SDS-PAGE)}

Proteins were separated by denaturing SDS-PAGE according to Laemmli (Laemmli, 1970) Protein samples were supplemented with $6 \mathrm{x}$ SDS loading dye and denatured for $5 \mathrm{~min}$ at $95^{\circ} \mathrm{C}$ before loading. The separating gel included $10 \%$ polyacrylamide $(30 \%, 37.5: 1)$ and the stacking gel comprised $4 \%$ polyacrylamide. The gel was run in $1 \mathrm{x}$ SDS running buffer at $60-120 \mathrm{~V}$. Proteins were visualized by Coomassie staining or transferred to a membrane for immunoblotting.

Table 2.10: Compositions of SDS gels

\begin{tabular}{lrrr} 
Component & Stock conc. & Stacking gel & Separating gel \\
\cline { 2 - 3 } Acrylamide & $30 \%$ & $4 \%$ & $10 \%$ \\
Tris-HCl, pH 6.8 & $1 \mathrm{M}$ & $125 \mathrm{mM}$ & - \\
Tris-HCl, pH 8.8 & $1 \mathrm{M}$ & - & $375 \mathrm{mM}$ \\
SDS & $10 \%(\mathrm{w} / \mathrm{v})$ & $0.1 \%$ & $0.1 \%$ \\
APS & $20 \%(\mathrm{w} / \mathrm{w})$ & $0.1 \%$ & $0.14 \%$ \\
TEMED & $100 \%$ & $0.1 \%$ & $0.07 \%$
\end{tabular}

In some cases, for instance for mass spectrometry, proteins were separated on 4-12\% NuPAGE ${ }^{\mathrm{TM}}$ gradient gels (Thermo Fisher Scientific) and run in 1x MOPS buffer at $200 \mathrm{~V}$. 


\section{Materials and Methods}

\subsubsection{Coomassie staining}

Coomassie staining of proteins was performed according to Sambrook et al. (Sambrook et al., 1989). The protein gel was fixed with gel-fixing solution for $30 \mathrm{~min}$, soaked in water for $3 \times 10 \mathrm{~min}$ and then stained with hot Coomassie staining solution for $30 \mathrm{~min}$. The gel was then de-stained in water until clear bands were visible.

\subsubsection{Western blot}

For western blot analysis, the denaturing SDS gel was transferred to a nitrocellulose membrane (Protan BA83 nitrocellulose, $0.2 \mu \mathrm{m}$ ) using the wet-tank transfer procedure. For the assembly of the transfer sandwich 6 Whatman papers and the membrane were saturated with $1 x$ western blot transfer $+20 \%$ methanol and assembled as follows: 3 Whatman papers, gel, membrane and again 3 Whatman papers. The electro transfer was conducted at $60 \mathrm{~V}(400$ $\mathrm{mA}$ max.) with the Bio-Rad Mini Trans-Blot Electrophoretic Transfer Cell system for $2 \mathrm{~h}$ at $4{ }^{\circ} \mathrm{C}$. The membrane was subsequently blocked for $1 \mathrm{~h}$ with $5 \%$ milk in $1 \mathrm{x}$ TBS-T, which also served as antibody solution. Immunoblotting was performed for at least $1 \mathrm{~h}$ (or overnight) at room temperature at $4{ }^{\circ} \mathrm{C}$ by using the primary antibodies listed in Section 2.1.9. The blot was washed 3 times for $10 \mathrm{~min}$ each with $1 \mathrm{x}$ TBS-T and subsequently incubated with the secondary antibody for $1 \mathrm{~h}$ at room temperature. As secondary antibody horseradish-peroxidase-conjugated antirabbit $(1: 50,000)$ or anti-mouse $(1: 10,000)$ antibodies were used. After 3 washes with 1x TBST the proteins were detected by enhanced chemiluminescence using the ECL detection kit (Amersham) as described in the user's manual.

In order to re-blot a membrane with other antibodies, previously blotted antibodies were stripped by incubation in western-blot stripping solution for $20-30 \mathrm{~min}$ at $50^{\circ} \mathrm{C}$. The membrane was then rinsed twice with water and washed 3 times with $1 \mathrm{x}$ TBS-T for $10 \mathrm{~min}$ at room temperature. Afterwards, the membrane was blocked again and subsequently incubated with new antibodies.

\subsubsection{Northern blot}

\section{Northern probe preparation}

The RNA was detected with $\left[\alpha^{32} \mathrm{P}\right]-\mathrm{dATP}(3000 \mathrm{Ci} / \mathrm{mmol})$ radioactively labelled probes. For probe generation the Prime It II Random Primer Labelling Kit (Stratagene) was used according to the manufacturers' instructions. PCR synthesized DNA-oligonucleotides hybridizing with one of the five snRNAs served as templates. 


\section{Materials and Methods}

\section{Northern blotting}

For northern blot analysis, samples were separated by denaturing polyacrylamide-gel electrophoresis and transferred to a nylon membrane by wet transfer (Trans-Blot Electrophoretic Transfer Cell). The transfer sandwich was assembled from 2 sheets of Whatman paper, gel, membrane and again 2 sheets of Whatman paper. Whatman papers and membrane had previously been soaked in northern Transfer Buffer. The transfer was carried out overnight at $20 \mathrm{~V}$ in a cold room. Subsequently, RNAs were crosslinked to the membrane by UV irradiation in a UVStratalinker 2400 (Auto Crosslink) with an energy of $1200 \mathrm{~J} / \mathrm{cm}^{2}$. Pre-hybridization was performed for $4 \mathrm{~h}$ at $42^{\circ} \mathrm{C}$ in $20 \mathrm{~mL}$ northern blot hybridization solution supplemented with $0.2 \mathrm{mg} / \mathrm{mL}$ denatured fish sperm DNA. The labelled probes were boiled at $95{ }^{\circ} \mathrm{C}$ for $5 \mathrm{~min}$ and together with fresh denatured fish-sperm DNA added to $20 \mathrm{~mL}$ northern blot hybridization solution. Hybridization was performed over night at $42{ }^{\circ} \mathrm{C}$. After collection of the Northern blot hybridization solution the membrane was washed 3 times with 20-25 mL pre-warmed northern blot wash buffer for $30 \mathrm{~min}$. The membrane was subsequently sealed in plastic foil. Signal visualization was performed by autoradiography.

\subsubsection{Special methods}

\subsubsection{Preparation of splicing active HeLa nuclear extract}

Nuclear extracts were prepared from HeLa cells essentially as described by Dignam et al. (Dignamr et al., 1983). The extracts (in Roeder $\mathrm{C}$ buffer) were subsequently frozen in liquid nitrogen and stored at $-80{ }^{\circ} \mathrm{C}$. For use in splicing reactions, the nuclear extracts were thawed and dialyzed against 50-100 volumes of Roeder D buffer for $2 \times 2.5 \mathrm{~h}$. To eliminate precipitate, the extracts were centrifuged at $8330 \mathrm{rpm}$ at $4{ }^{\circ} \mathrm{C}$ for $10 \mathrm{~min}$ in $\mathrm{F} 14-14 \times 50 \mathrm{cy}$ rotor (centrifuge Sorvall ${ }^{\circledR}$ Evolution RC Lynx 6000) and aliquots of the supernatants were frozen in liquid nitrogen to be stored at $-80^{\circ} \mathrm{C}$.

\subsubsection{Transient transfection with siRNA and cell fractionation}

HeLa SS6 cells were grown in $10 \mathrm{~cm}$ dishes containing Dulbecco's Modified Eagle's Medium (DMEM) supplemented with $10 \%$ fetal bovine serum (FBS) and $100 \mu \mathrm{g} / \mathrm{mL}$ penicillin/streptomycin. Cell cultures were kept at at $37^{\circ} \mathrm{C}$ in $5 \% \mathrm{CO}_{2}$. For cell-culture maintainance, the cells were washed twice with $1 \mathrm{x}$ PBS and dissociated from the dish by addition of Accutase ${ }^{\circledR}$ solution. The cells were then resuspended in fresh DMEM.

For transient transfection with siRNAs, DMEM supplemented with $10 \%$ FBS but without antibiotics was used. About $3 \times 10^{5}$ cells were cultured in 6 wells one day before transfection. For transfection the Lipofectamine ${ }^{\circledR}$ RNAiMAX Transfection Reagent (Thermo Fisher Scientific) was used as described in the user's manual. After a transfection time of 60 hours, cells were washed twice with ice-cold 1x PBS and then resuspended to $\sim 10 \times 10^{6}$ cells $/ \mathrm{mL}$ into HeLa Cells Lysis Buffer using a sterile cell scraper. After brief vortexing, the samples were incubated on ice for 15 $\min$. The nucleoplasmic fraction was separated from the chromatin faction by centrifugation at $4{ }^{\circ} \mathrm{C}$ and $1100 \times \mathrm{g}$ 


\section{Materials and Methods}

for $10 \mathrm{~min}$. The soluble nucleoplasmic fraction was collected and the chromatin faction resuspended in a volume of HeLa Cell Lysis Buffer equivalent to the nucleoplasmic fraction. In a $1.5 \mathrm{~mL}$ reaction tube, the chromatin was solubilized by sonication in a Bioruptor for $3 \mathrm{~min}$ (30 sec on, $30 \mathrm{sec}$ off) at maximum intensity in a water bath at $2^{\circ} \mathrm{C}$.

The protein concentration was determined using the BCA Protein Assay Kit (Thermo Fisher Scientific) according to the user's manual. The protein composition was analysed by SDS-PAGE followed by western blotting.

\subsubsection{RNA seq and data-processing}

For RNA seq analysis, HeLa cells were transfected with siRNAs specific for Smu1, RED or MFAP1 as described in Section 2.2.3.2. As control, an siRNA without target in the human transcriptome was transfected. Cells were harvested 50 hours after transfection and their total RNA was extracted. The RNA was further processed by the laboratory of Juan Valcárcel; this comprised reverse transcription of the RNA, Illumina sequencing and data analysis.

The reads obtained by Hiseq-Illumina Paired End sequencing (100bp reads post adapter/barcode removal) were mapped on the human hg19 genome and the UCSC transcriptome by using TopHat2 (v2.0.8). Differential transcript splicing was quantified by using the Cuffdiff program of the Cufflinks suite (v2.1.1) and alternative splicing events, including usage of alternatively spliced exons, alternative 5'SS, alternative 3'SS and retained introns were quantified by using the MISO package (v0.5.2).

\subsubsection{Insect-cell expression of recombinant proteins}

Smu1 and RED were expressed separately or jointly in SF-9 or High Five cells using synthetic genes optimized for expression (GeneArt, Life Technologies). For Baculovirus generation, the Smu1 and RED full-length (FL) or truncation $(\Delta)$ constructs were amplified by PCR using a primer comprising the required restriction sites. It was subsequently inserted into the pFL vector. To co-purify the dimers, RED was tagged with an N-terminal poly-His 10 tag under control of the polyhedrin (polh) promoter (EcoRI/Pstl) while Smu1 was cloned with a C-terminal Strep-II tag (from provider) under control of the $\mathrm{p} 10$ promoter (Xhol/Nsil). Standard digest and ligation reaction are illustrated in Tables 2.3 and 2.4. Since Strep-II tag purification proved to be inefficient, Smu1-FL was additionally cloned with an $\mathrm{N}$-terminal $\mathrm{His}_{10}$ tag (EcoRI/Pstl) for single purification. For amplification, obtained vectors were transfected into chemically competent E. coli XL-10 Gold cells. The entire ligation was incubated with $100 \mu \mathrm{L}$ cells on ice for $30 \mathrm{~min}$. Heat shock was performed at $42{ }^{\circ} \mathrm{C}$ for $1 \mathrm{~min}$, followed by a $5 \mathrm{~min}$ incubation on ice. For recovery $1 \mathrm{~mL}$ LB was added to the cells, which were subsequently incubated in a thermomixer at $37^{\circ} \mathrm{C}$ and $300 \mathrm{rpm}$ for $1 \mathrm{~h}$. Cells were then plated on LB plates containing $100 \mu \mathrm{g} / \mathrm{mL}$ ampicillin. 


\section{Materials and Methods}

Plasmids were isolated using the High Pure Plasmid Isolation Kit from Roche or the PureLink ${ }^{\mathrm{TM}} \mathrm{HiPure} \mathrm{Plasmid}$ Filter Maxiprep Kit from Thermo Fisher Scientific as described in the manufacturers protocol. The sequence was verified by Sanger sequencing (Microsynth Seqlab).

Errorless vectors were transformed into DH10MultiBacY cells for inclusion into baculoviral DNA. For that purpose, $600 \mathrm{ng}$ of vector was added to $100 \mu \mathrm{L}$ of electro-competent $\mathrm{DH} 10$ MultiBacY cells and incubated on ice for $30 \mathrm{~min}$. The cells were transferred to an electroporation cuvette and a single $2000 \mathrm{~V}$ pulse was applied. Immediately, $1 \mathrm{~mL}$ of LB medium was added to the cells, which were transferred to a Greiner culture tube and incubated in a Multitron incubator shaker (Infors HT) at $37^{\circ} \mathrm{C}$ and $180 \mathrm{rpm}$ overnight. The transformation products were plated onto LBAgar containing $100 \mu \mathrm{g} / \mathrm{mL}$ ampicillin, $10 \mu \mathrm{g} / \mathrm{mL}$ gentamycin, $50 \mu \mathrm{g} / \mathrm{mL}$ kanamycin, $12 \mu \mathrm{g} / \mathrm{mL}$ tetracycline, $1 \mathrm{mM}$ IPTG and $150 \mu \mathrm{g} / \mathrm{mL}$ X-gal. Overnight cultures in LB Medium, containing only antibiotics, were prepared from positive (white) colonies. Viral plasmids were isolated using the High Pure Plasmid Isolation Kit from Roche according to the user's manual until step 4. This means that after removal of the precipitate the supernatant was not transferred to the High Pure Filter Tube, but supplemented with $700 \mu \mathrm{L}$ isopropanol. The plasmid was precipitated at $-20{ }^{\circ} \mathrm{C}$ for at least $30 \mathrm{~min}$ and the DNA was collected by centrifugation at $13,000 \mathrm{rpm}$ and $4{ }^{\circ} \mathrm{C}$ for $15 \mathrm{~min}$ in a microfuge. After one wash with $75 \%$ ethanol the pellet was dissolved in $20 \mu \mathrm{L}$ deionized and autoclaved water. 6 well plates, each well containing $3 \mathrm{~mL}$ of $0.3 \times 10^{6} \mathrm{Sf} 9$ cells $/ \mathrm{mL}$ in Gibco ${ }^{\circledR}$ Sf- $900^{\mathrm{TM}}$ III SFM medium, were prepared and transfected with the isolated plasmid using X-tremeGENE ${ }^{\text {TM }} 9$ DNA Transfection Reagent according to the manufacturers protocol. $60-72 \mathrm{~h}$ after transfection at $27^{\circ} \mathrm{C}$ (KB incubator), the virus containing supernatant was collected $\left(V_{0}\right)$. Fresh Sf9 cells, maintained in suspension in Gibco ${ }^{\circledR}$ Sf-900 9 TM III SFM medium at $0.7 \times 10^{6}$ cells $/ \mathrm{mL}$ were transfected to generate the $\mathrm{V}_{1}$ virus and incubated at $25{ }^{\circ} \mathrm{C}$ and $90-100 \mathrm{rpm}$ on a MaxQ 3000 Benchtop Shaker. For protein expression, the optimized High Five ${ }^{\mathrm{TM}}$ cell line was used. The cells were maintained in suspension in ESF 921 Insect Cell Culture Medium at $0.7 \times 10^{6}$ cells $/ \mathrm{mL}, 25^{\circ} \mathrm{C}$ and $90-100 \mathrm{pm}$. The cells were harvested $60-72 \mathrm{~h}$ after transfection by centrifugation at $4{ }^{\circ} \mathrm{C}$ and $3000 \mathrm{rpm}$ for $10 \mathrm{~min}$ using a Multifuge $1 \mathrm{~S}-\mathrm{R}$ and either immediately subjected to protein purification or frozen in liquid nitrogen and stored at $-80{ }^{\circ} \mathrm{C}$.

\subsubsection{Purification of recombinant proteins from insect cells}

The High Five ${ }^{\mathrm{TM}}$ cell pellet was resuspended in $10 \mathrm{~mL} / \mathrm{g}$ cells of pre-chilled High Five Lysis Buffer and disrupted by ultrasonic probe sonication at 30\% amplitude for 15-30 min (30 msec on, $30 \mathrm{msec}$ off) until the lysate was clear. After centrifugation for $1 \mathrm{~h}$ at $4{ }^{\circ} \mathrm{C}$ and $10,000 \mathrm{rpm}$ in a F14-14 x 50cy rotor (Sorvall LYNX 6000 Superspeed Centrifuge) the soluble cell fraction was diluted 1:1 with High Five Dilution Buffer and incubated with previously equilibrated Roti ${ }^{\circledR}$ garose-His/Ni Beads (Roth) at $4{ }^{\circ} \mathrm{C}$ for $3 \mathrm{~h}$ with head-over-tail rotation. The beads were collected and washed three times with High Five Wash Buffer for 10 minutes at $4{ }^{\circ} \mathrm{C}$ with head-over-tail rotation. Elution was performed with 1.5-3 bead volumes High Five Elution Buffer on ice for 15 minutes. Eluates were frozen in liquid nitrogen and stored at $-80{ }^{\circ} \mathrm{C}$. Analysis of the purified proteins was performed by SDS polyacrylamide gelelectrophoresis, followed by staining with Coomassie blue. 


\section{Materials and Methods}

\subsubsection{Crosslinking of antibodies to Dynabeads ${ }^{\mathrm{TM}}$ Protein $A$}

In order to obtain antibodies covalently associated with beads, antibodies were crosslinked to the beads by using dimethyl pimelimidate (DMP). The crosslinking procedure is based on the protocol provided by Abcam.

$600 \mu \mathrm{L}$ beads were washed 3 times with 1x PBS head-over-tail at $4{ }^{\circ} \mathrm{C}$ for at least $15 \mathrm{~min} .50 \mathrm{mg}$ of anti-Smu1 antibody in $800 \mu \mathrm{L}$ PBS was added to the beads and incubated overnight with head-over-tail rotation at $4{ }^{\circ} \mathrm{C}$. As a control (mock) another set of beads was incubated with buffer without any antibody. The following steps were performed with head-over-tail rotation at room temperature. The beads were washed 3 times with $1 \times$ PBS for 10 min and subsequently equilibrated with triethanolamine solution for 2 times $10 \mathrm{~min}$. The DMP was dissolved in a triethanolamine solution to a concentration of $25 \mathrm{mM}$. Crosslinking was performed with $1 \mathrm{~mL} 25 \mathrm{mM}$ DMP for $1 \mathrm{~h}$ and another $30 \mathrm{~min}$ with fresh DMP. The crosslinker was quenched by incubation with $2 \mathrm{x} 50 \mathrm{mM}$ Tris, $\mathrm{pH} 7.5$ in $1 \mathrm{x}$ PBS for $10 \mathrm{~min}$.

After two washes with PBS, the excess of antibody was removed by incubation in $200 \mathrm{mM}$ glycine, pH 2.5 for 2 $\min$. The beads were subsequently washed three times with $1 \mathrm{x}$ PBS-T for $10 \mathrm{~min}$. The beads were stored in $1 \mathrm{x}$ PBS-T containing additionally $0.09 \%$ sodium azide.

\subsubsection{Immunodepletion of HeLa nuclear extract}

Immunodepletion of Smu1/RED from HeLa nuclear extract (Section 2.2.3.1) was performed using anti-peptide antibodies specific for Smu1. The crosslinked beads were washed 3 times with 1x PBS and then blocked overnight in 1x PBS supplemented with $0.5 \mathrm{mg} / \mathrm{mL}$ BSA, $0.05 \mathrm{mg} / \mathrm{mL}$ tRNAE.c.and $0.05 \mathrm{mg} / \mathrm{mL}$ Glycogen.

To minimize unspecific binding to the beads, the salt concentration of the nuclear extract was increased to $750 \mathrm{mM}$ $\mathrm{KCl}$ (SE-750). After three washes with Roeder D buffer containing an increased salt concentration of $750 \mathrm{mM} \mathrm{KCl}$ (RD-750 buffer), the beads were divided into two equal portions and the SE-750 was incubated successively with each portion for $2 \mathrm{hr}$ at $4{ }^{\circ} \mathrm{C}$ with head-over-tail rotation. The extract was subsequently dialyzed against RD-100 buffer for $5 \mathrm{~h}$ at $4{ }^{\circ} \mathrm{C}$. Mock-depleted extract was treated in a similar manner while the antibody was omitted.

\subsubsection{In vitro splicing reaction}

A typical splicing reaction was carried out in the presence of $10 \mathrm{nM}$ MINX, PM5 or PM5-10 pre-mRNA and $40 \%$ $(\mathrm{v} / \mathrm{v})$ HeLa nuclear extract or $50 \%$ depleted extract. The composition of a standard splicing reaction is illustrated in Table 2.11.

The splicing reaction mixtures were incubated at $30{ }^{\circ} \mathrm{C}$ for the times indicated in the respective figure (Section 3 , Results). 


\section{Materials and Methods}

Table 2.11: Composition of a standard in vitro splicing reaction

\begin{tabular}{lrr} 
Component & Stock conc. & Final conc. \\
\cline { 2 - 3 } & $1 \mathrm{M}$ & $20 \mathrm{mM}$ \\
$\mathrm{MgCl}_{2}$ & $500 \mathrm{mM}$ & $3 \mathrm{mM}$ \\
$\mathrm{KCl}$ & $3000 \mathrm{mM}$ & $60 \mathrm{mM}$ \\
ATP & $100 \mathrm{mM}$ & $2 \mathrm{mM}$ \\
Creatine phosphate & $500 \mathrm{mM}$ & $20 \mathrm{mM}$ \\
Nuclear extract & & $40-50 \%$ \\
$\left({ }^{32} \mathrm{P}-\right)$ pre-mRNA & & $5-10 \mathrm{nM}$
\end{tabular}

For in vitro trans-splicing, $10 \mathrm{nM}$ of the $5^{\prime}$ fragment and $50 \mathrm{nM}$ of the 3 ' fragment were assembled in a standard splicing reaction.

To assemble spliceosomes in the presence of recombinant Smu1 and/or RED proteins, the nuclear extract was first supplemented with a 30 -fold excess of recombinant protein and pre-incubated for 15 min at $30{ }^{\circ} \mathrm{C}$ before addition to the splicing reaction.

For chase experiments, the splicing reaction was incubated for the time indicated and the recombinant protein was then added along with a 10-fold excess of unlabelled pre-mRNA. To exclude the risk that an effect might have been due to the increased salt concentration or the presence of imidazole, a control was performed using buffer without protein.

\subsubsection{Analysis of in vitro splicing by Denaturing PAGE}

In order to analyse the products of in vitro pre-mRNA splicing, the splicing reaction was subjected to PK digestion followed by $\mathrm{PCl}$ extraction, and the RNA isolated was resolved on a denaturing polyacrylamide gel. To visualize pre-mRNA, splicing intermediates and products, the gel was dried at $70{ }^{\circ} \mathrm{C}$ for $2 \mathrm{~h}$ and visualised with a phosphorimager screen.

\subsubsection{Analysis of splicing complexes by native agarose gel-electrophoresis}

The assembly of the spliceosome in vitro can be analysed by using a mobility-retardation assay, since the A, B Bact and C complexes exhibit a decreased mobility on native agarose gels (Lamond et al., 1987; Kent \& MacMillan, 2002). To that end, splicing reaction mixtures were supplemented with $0.5 \mu \mathrm{g} / \mu \mathrm{L}$ heparin and $5 \mathrm{x}$ native gel-loading dye before loading the sample onto a native gel $(2 \% \mathrm{w} / \mathrm{v}$ low-melting-point agarose in $1 \mathrm{x}$ TBE buffer). Electrophoresis was performed at 50-60 mA for $18 \mathrm{~h}$ at room temperature. The gels were subsequently dried at 60 ${ }^{\circ} \mathrm{C}$ for 3-4 $\mathrm{h}$ and visualised with a phosphorimager screen. 


\section{Materials and Methods}

\subsubsection{MS2 affinity-selection of splicing complexes}

The isolation of spliceosomal complexes was performed by MS2 affinity-selection as described previously (Bessonov et al., 2010). To allow purification, the pre-mRNA was pre-incubated with a 20 -fold molar excess of purified MS2-MBP fusion protein in the presence of $20 \mathrm{mM} \mathrm{HEPES-KOH,} \mathrm{pH} 7.9$ for 30 min at $4{ }^{\circ} \mathrm{C}$.

Subsequently, the pre-mRNA was added to a $1 \mathrm{~mL}$ standard splicing reaction. Kinetic and $\triangle$ Smu1/RED $B$ complexes were incubated for $8 \mathrm{~min}$ at $30^{\circ} \mathrm{C}$. Spliceosomes were assembled on MINX-70 for 15 minutes.

The splicing reaction was loaded onto a $14 \mathrm{ml}$ linear 10-30 \% (v/v) glycerol gradient containing G-150 buffer. Ultracentrifugation of the gradients was performed at $22,000 \mathrm{rpm}$ for $15 \mathrm{~h} 20 \mathrm{~min}$ at $4{ }^{\circ} \mathrm{C}$ in a Sorvall TST 41.14 rotor, and the $500 \mu \mathrm{l}$ gradient fractions were harvested manually from top to bottom. The distribution of ${ }^{32} \mathrm{P}$-labeled pre-mRNA was analysed by Cherenkov counting.

For affinity selection, the peak fractions containing the respective spliceosomal complexes were pooled and loaded onto a pre-equilibrated column containing $200 \mu$ of packed amylose beads (NEB) and incubated for 15 min. The matrix was then washed with 50 column volumes of G-75 buffer. Elution of the spliceosomal complexes was performed by addition of $400 \mu \mathrm{l}$ of G-75 buffer containing $25 \mathrm{mM}$ maltose. The snRNA composition was analysed by denaturing polyacrylamide-gel electrophoresis after PK digestion and $\mathrm{PCl}$ extraction or by submission to mass spectrometry.

\subsubsection{Mass spectrometry}

The protein composition of spliceosomal complexes was analysed by mass spectrometry. To this end, isolated spliceosomal complexes were concentrated by ethanol precipitation (addition of 0.1 volume $3 \mathrm{M} \mathrm{NaOAC} \mathrm{pH} 5$ and 5 volumes absolute ethanol) at $-80^{\circ} \mathrm{C}$ for $48 \mathrm{~h}$. The RNP pellet obtained was dissolved in $1 \times$ NuPAGE ${ }^{\text {TM }}$ LDS Sample Buffer, supplemented with NuPAGE ${ }^{\mathrm{TM}}$ Sample Reducing Agent and denatured at $70{ }^{\circ} \mathrm{C}$ for $10 \mathrm{~min}$. The proteins were separated on 4-12\% NuPAGE ${ }^{\mathrm{TM}}$ gradient gels (Invitrogen) and stained with Coomassie blue.

Each lane was divided into 23 slices and the proteins of each slice were digested in-gel with trypsin and extracted as described by Shevchenko et al. (Shevchenko et al., 1996). The extracted peptides were subsequently analyzed in a $Q$ Exactive ${ }^{\mathrm{TM}} \mathrm{HF}$ Hybrid Quadrupol-Orbitrap ${ }^{\mathrm{TM}}$ Mass Spectrometer (Thermo Fisher Scientific) under standard conditions. Proteins were identified by searching fragment spectra against the UniProt data base. Sample preparation and mass spectrometric analysis was performed by the laboratory of Henning Urlaub. 


\section{Materials and Methods}

\subsubsection{Purification of endogenous spliceosomes from the chromatin fraction}

\section{Crosslinking and sonication}

For the purification of spliceosomal complexes from the chromatin fraction, cells were lysed as described in Section 2.2.3.2. The HeLa Cell Lysis Buffer, however, contained $30 \mathrm{mM}$ HEPES-KOH pH 7.5 instead of Tris- $\mathrm{HCl}$ and the chromatin was pelleted by centrifugation at $700 \times \mathrm{g}$ at $4{ }^{\circ} \mathrm{C}$ for $10 \mathrm{~min}$. The chromatin pellet was resuspended in a volume of HeLa W/O Salt Buffer equivalent to the lysis volume. The chromatin was then passed through a $26 \mathrm{G}$ needle until homogenized. For crosslinking dithiobis succinimidyl propionate (DSP) was dissolved in dimethyl sulfoxide (DMSO) and added to a final concentration of $1.5 \mathrm{mM}$. Crosslinking was performed on ice, at $4{ }^{\circ} \mathrm{C}$ for 1 h. An equal volume of HeLa Sonication Buffer was added and $250 \mu \mathrm{L}$ per $1.5 \mathrm{~mL}$ reaction tube was sonicated in a Bioruptor at maximum intensity ( $30 \mathrm{sec}$ on, $30 \mathrm{sec}$ off) in a $0.5^{\circ} \mathrm{C}$ cold water bath. Sonication was performed for 2 times $60 \mathrm{~min}$ with an incubation on ice for $30 \mathrm{~min}$ in between.

\section{Glycerol-gradient sedimentation}

The cross-linked and sonicated samples were layered on a 10-40\% glycerol gradient containing $65 \mathrm{mM} \mathrm{NaCl}, 30$ $\mathrm{mM}$ Tris-HCl pH 7.5 and $1 \mathrm{M}$ EDTA. Centrifugation was performed at $4{ }^{\circ} \mathrm{C}$ and 60,000 rpm in a Th660 (Thermo scientific) rotor for $1 \mathrm{~h}$ (Sorvall WX Ultracentrifuge). The gradient was fractionated into $175 \mu \mathrm{L}$ fractions, which were subsequently analyzed by western blotting. For crosslink reversal 3x loading dye containing $180 \mathrm{mM}$ Tris, $6 \%$ SDS, $300 \mathrm{mM}$ DTT and bromophenol blue was mixed with the samples and the mixture was kept at $95^{\circ} \mathrm{C}$ for $7 \mathrm{~min}$. From the fractions of interest P-Prp31- and P-SF3b155-associated spliceosomes were purified by immunoprecipitation.

\section{Immunoprecipitation}

For immunoprecipitation the peak fractions were pooled and diluted 1:1 with HeLa IP Dilution Buffer.

Unless otherwise stated, incubations were performed at $4{ }^{\circ} \mathrm{C}$ under rotation. $\sim 5 \mu \mathrm{g}$ of the purified antibodies were directly added to the diluted fractions, incubated at $4{ }^{\circ} \mathrm{C}$ for $2 \mathrm{~h}$ and then with $\sim 30 \mu \mathrm{L}$ protein $\mathrm{A}$ Sepharose beads. After precipitation the beads were sedimented by centrifugation at 3,000 rpm for $3 \mathrm{~min}$ in a Biofuge fresco (Heraeus) and the supernatant was collected for further investigation. The beads were washed 4x with HeLa IP Wash Buffer followed by incubation on ice for $2 \mathrm{~min}$. The precipitate's RNA content was analysed by northern blotting. 


\section{Results}

The spliceosome is characterised by its highly complex and dynamic structure and composition. While lower eukaryotes get by with the core components necessary for intron excision, higher eukaryotes require additional factors to cope with the increased challenges of a more complex gene architecture. Smu1 and RED are two nonconserved spliceosomal factors whose function in splicing is currently poorly understood. In order to shed some light on this issue, this study targets the role of Smu1 and RED in splicing with the help of a combination of genome wide, in vivo and in vitro approaches.

\subsection{Alternative and constitutive splicing are affected by knockdown of Smu1 and RED}

Several studies targeting Smu1 and RED have revealed that their knock-down/gene mutation affects alternative splicing in plants, worms and humans (Spartz et al., 2004; Chung et al., 2009; Papasaikas et al., 2015). However, as these studies focused on known alternative splicing events, the question of whether constitutive splicing is altered in the absence of Smu1 and RED has not been investigated. To address this issue, I examined how their depletion generally effects splicing in HeLa cells by RNA-seq analysis. To be able to differentiate between Smu1/RED-specific effects on splicing and effects shared with other B-specific proteins, the knock-down of MFAP1 was additionally investigated. For that purpose, HeLa cells were transfected with siRNAs specific for Smu1, RED or MFAP1, respectively. As a control, an siRNA without target in the human transcriptome was transfected. $50 \mathrm{~h}$ after transfection the cells were harvested and the total RNA was extracted for Illumina sequencing. The RNAseq analysis was performed by Panagiotis Papasaikas in the laboratory from Juan Juan Valcárcel (CRG, Bercelona).

Hiseq-Illumina Paired End sequencing (100bp reads post adapter/barcode removal) generated 2 × 135,376,977 reads for the control, $2 \times 144,695,392$ reads in $\triangle$ RED cells, $2 \times 129,201,923$ reads in $\Delta$ Smu1 cells and $2 \times$ $131,781,731$ reads in $\triangle M F A P 1$ cells. About $89 \%, 88 \%, 86 \%$ and $85 \%$ of the total reads for the control, $\triangle R E D$, $\triangle$ Smu1 and $\triangle$ MFAP1 cells, respectively, were mapped on the human hg19 genome and the UCSC transcriptome by using TopHat2 (v2.0.8). Differential transcript splicing was quantified by using the Cuffdiff program of the Cufflinks suite (v2.1.1) and alternative splicing events, including usage of alternatively spliced exons, alternative 5'SS (Alt5'SS), alternative 3'SS (Alt3'SS) and retained introns were quantified by using the MISO package (v0.5.2). As shown in Fig. 3.1A, the alternative splicing of cassette exons and intron retention represented the majority of changes in splicing events upon depletion of Smu1, RED and MFAP1. A substantial majority of cassette exons (Cex) were skipped (Fig. 3.1B). Usage of alternative 5'SS and 3'SS detected in Smu1 or RED depleted cells were largely overlapping ( $\sim 56 \%$ ) while the overlap level dropped to $35-40 \%$ between Smu1/RED and MFAP1 depleted cells (Fig. 3.1C and D). 


\section{Results}

Similarly, a high level of similarity (72\% overlap) between Smu1- and RED-depleted cells was observed for the alternative splicing of cassette exons. This observation is consistent with the idea that the two factors function as a dimer. In comparison, the extent of overlap observed for cells depleted of Smu1 or RED with $\triangle$ MFAP1 cells was reduced to $51 \%$ (Fig. 3.1E). Nevertheless, a similarity of $51 \%$ is not a small matter and is indicative of factors that associate concomitantly with the spliceosome.

A

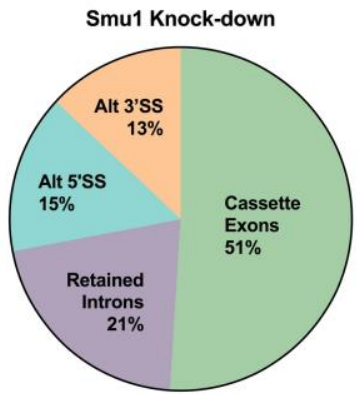

B
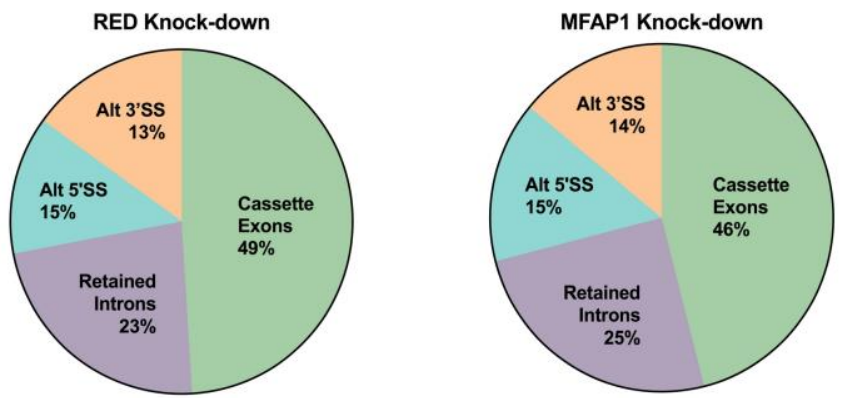

Fraction of cassette exon inclusion or skipping

G Fraction of constitutively spliced introns
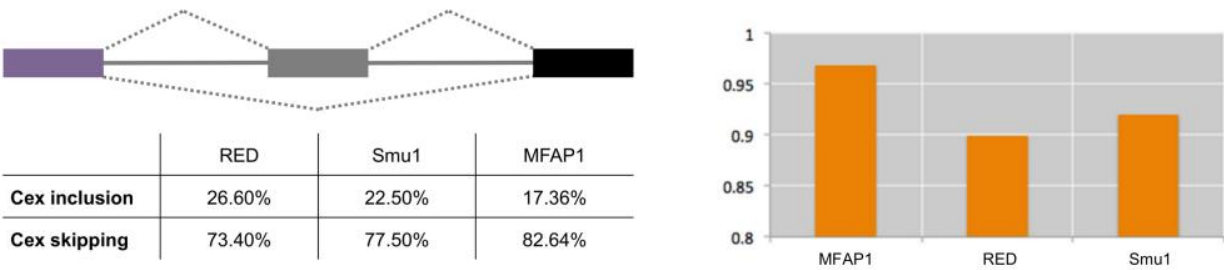

C

Alternative 3' splice site

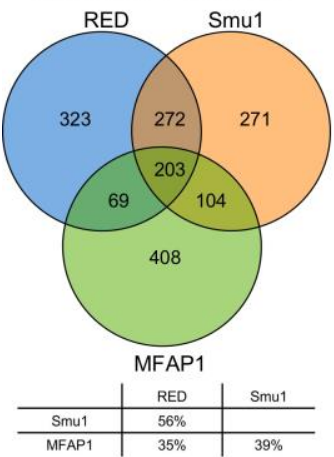
Alternative $5^{\prime}$ splice site

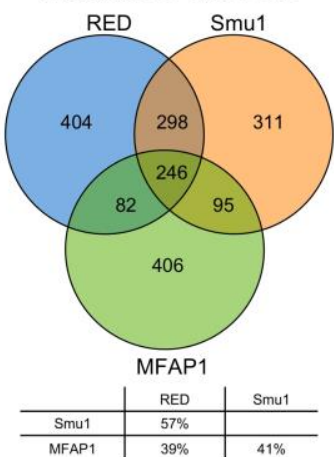

E

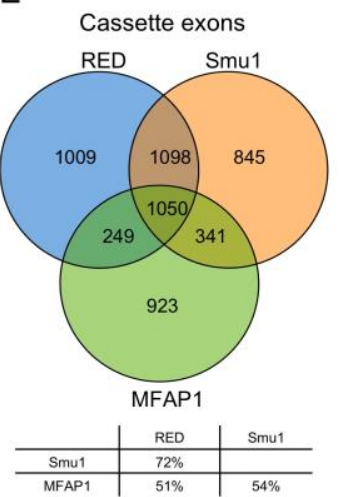

$\mathrm{F}$

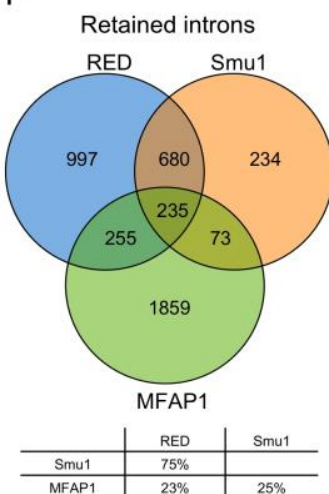

Figure 3.1. Splicing events effected by siRNA-mediated depletion of Smu1, RED or MFAP1. (A) Pie charts showing class distribution of all alternative splicing events detected: Usage of alternatively spliced cassette exons, alternative 5'SS (Alt5'SS), alternative 3'SS (Alt3'SS) and retained introns in siRNA-mediated knock-down cells for Smu1, RED and MFAP1. (B) Relative fraction of cassette exon (Cex) inclusion or skipping among the population of cassette exons whose alternative splicing is affected in $\triangle \mathrm{Smu} 1, \triangle \mathrm{RED}$ and $\triangle \mathrm{MFAP} 1$ HeLa cells. (C-F) Venn diagrams showing the extent of the AS events overlap between each class of AS, i.e. alternative 3' splice sites (C), alternative 5' splice sites $(\mathrm{D})$, cassette exon $(\mathrm{E})$ and retained introns $(\mathrm{F})$ in the three different cell types i.e. $\triangle \mathrm{Smu} 1, \triangle \mathrm{RED}$ and $\triangle \mathrm{MFAP} 1$ HeLa cells. Numbers represented in the circles represent the absolute number of the corresponding AS events detected in each depletion experiment. The table shows the relative overlap in \%. (G) Chart representing the fraction of constitutively spliced introns among the introns retained (unspliced) in $\triangle \mathrm{Smu} 1, \triangle \mathrm{RED}$ and $\triangle \mathrm{MFAP} 1$ cells. 
As observed for cassette exons, introns retained upon Smu1 and RED depletion also showed a $75 \%$ overlap. Strikingly, the overlap of $\triangle$ Smu1/RED cells with MFAP1-depleted cells was (at $25 \%$ ) dramatically reduced, indicating that the majority of introns retained upon MFAP1 depletion were normally processed in Smu1/REDdepleted cells and vice versa (Fig. 3.1F). Introns retained in $\triangle$ RED, $\triangle$ Smu1 and $\triangle M F A P 1$ cells appeared to represent in their vast majority (>90\%) introns that are normally constitutively spliced (Fig. $3.1 \mathrm{G})$ by the spliceosome. This suggests that Smu1 and RED are important for splicing in general, and are not only involved in the regulation of alternative splicing.

\subsection{Short introns are predominantly retained upon knock-down of Smu1 and RED}
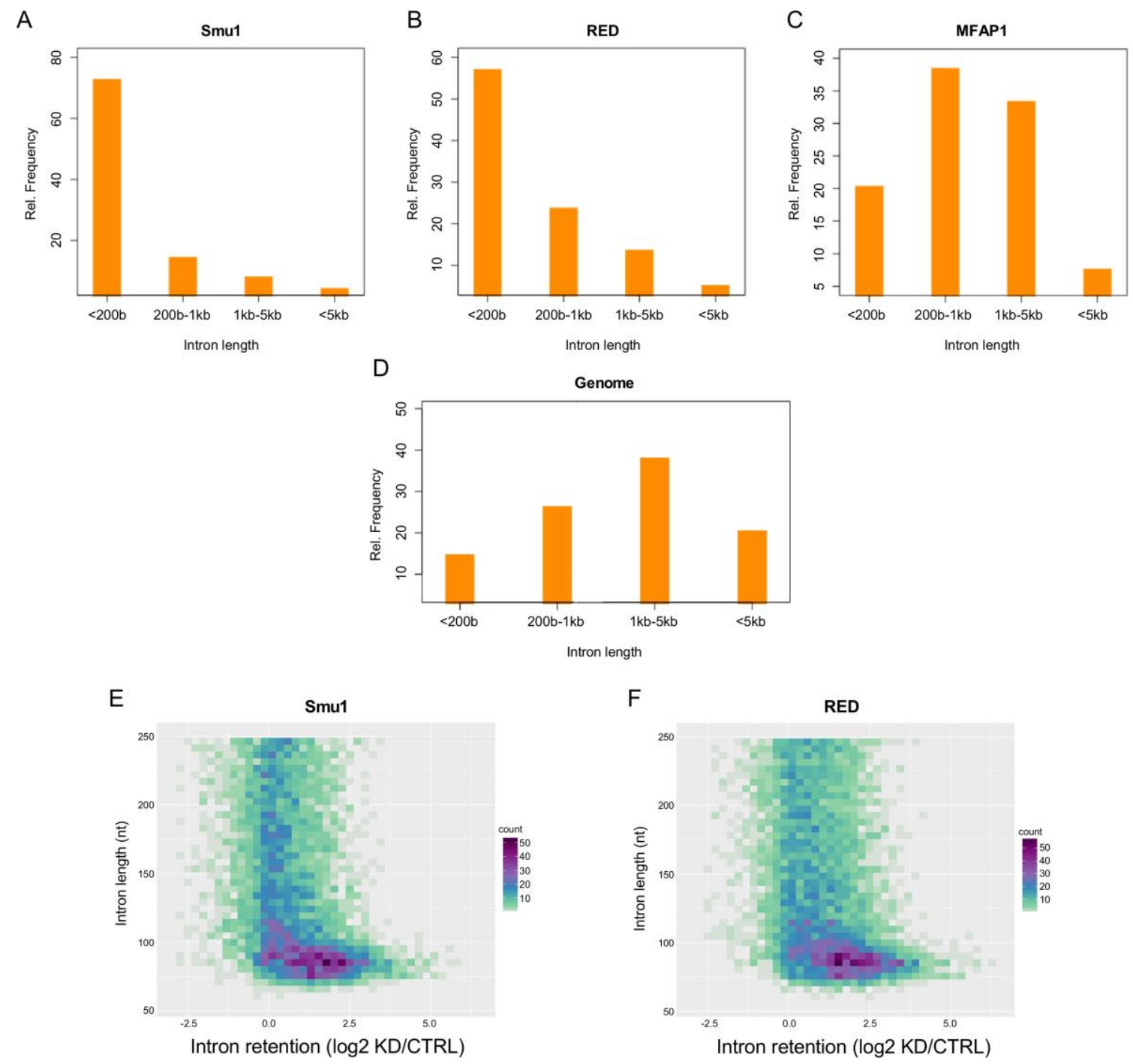

Figure 3.2. siRNA-mediated depletion of Smu1 or RED results in retention of short constitutively spliced introns. (A-C) Introns were sorted into 4 classes according to their length i.e. $<200$ bases, 200 to $1 \mathrm{~Kb}, 1 \mathrm{~Kb}$ to $5 \mathrm{~Kb}$ and $>5 \mathrm{~Kb}$. Chart showing the relative distribution among those 4 length classes of the introns retained in $\triangle$ Smu1(A), $\triangle R E D$ (B) and $\triangle M F A P 1$ (C) cells. (D) Chart showing the relative distribution of intron length (whole genome) among these 4 classes. (E \& F) Heat maps showing the level of intron retention ( $x$ axis) relative to the intron length ( $y$-axis) upon depletion of Smu1 $(E)$ or RED (F). The colour code ranges from pale green for low abundance introns to deep red for highly abundant introns (referred to as "count"). 


\section{Results}

All attempts to identify common features of the introns retained after Smu1 and RED knock-down, failed to reveal any mutual motives or sequences. However, surprisingly, I found that introns shorter than $200 \mathrm{nt}$ were preferentially retained. As shown in Fig. 3.2A and B, $\sim 60 \%$ of the introns retained in $\triangle$ Smu1 and $\triangle R E D$ cells exhibited a length under 200 nucleotides, even though such short introns only have a prevalence in the whole human genome of about 15\% (Fig. 3.2D). In comparison, MFAP1-depletion did not show any preference for short introns, as they represented only $20 \%$ of the introns retained (Fig. 3.2 C). Thus, defects in constitutive splicing showed a strong bias toward short introns in Smu1- and RED-depleted cells. Closer examination indicated that introns presenting the highest level of retention in $\triangle \mathrm{Smu} 1$ and $\triangle \mathrm{RED}$ cells had a strikingly homogenous length of around $75-100 \mathrm{nt}$

(Fig. 3.2E and F). This distribution supports the idea that intron length may be a co-determinant of the Smu1/RED requirement for efficient intron splicing.

\subsection{A Smu1-specific antibody effectively co-depletes Smu1 and RED from HeLa nuclear extract}

The RNAseq analysis of Smu1- or RED-depleted cells suggested that these two proteins are required for splicing of introns shorter than $100 \mathrm{nt}$. To recapitulate the effect seen in vivo and to dissect the roles of Smu1 and RED in pre-mRNA splicing, I set up a suitable in vitro system. As a first measure, I removed Smu1 from splicing-competent nuclear extract by immunodepletion with a Smu1-specific antibody. To exclude the possibility that an effect is due to the procedure (e.g. to the increase in salt concentration during the depletion, or to the exposure to beads) mockdepleted extract was prepared in exactly the same manner, but with omission of the antibody. This extract served as a control extract in the later splicing experiments. To further assess unspecific binding to the beads, SE750 (splicing extract with $750 \mathrm{mM} \mathrm{KCl}$ ) was also investigated; in this control the extract had a higher salt concentration but was not exposed to beads.

Analysis of the depleted extract by immunoblotting revealed that Smu1 was nearly completely depleted from the extract, along with around $90 \%$ of RED (Fig. 3.3A and B). Apparently, the physical interaction between these two proteins resulted in an efficient co-depletion of RED with Smu1, even at $1 \mathrm{M} \mathrm{KCl}$, underlining the stability of the Smu1/RED dimer. In contrast, other B-specific proteins (Prp38, MFAP1 and FBP21) and also core spliceosomal factors (U5-Snu114K, tri-snRNP protein Snu66 and U2-SF3a66) were not affected by the depletion or by contact with the beads, indicating that the Smu1-specific antibody indeed selectively depleted the Smu1/RED dimer from the extract. 
A

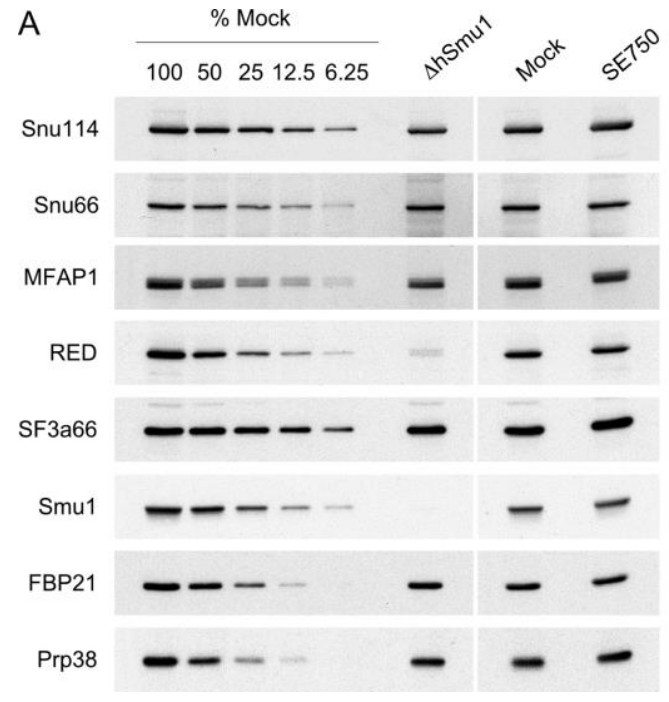

B

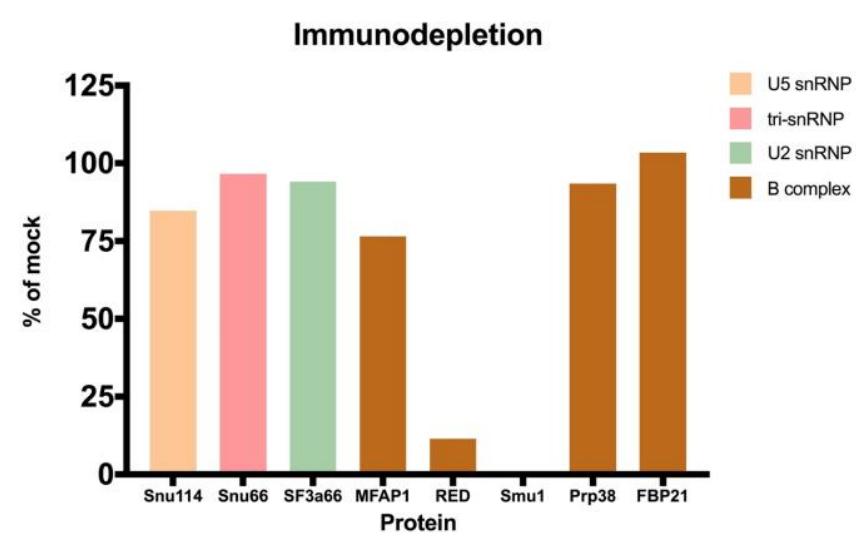

Figure 3.3. Smu1 and RED levels are efficiently and specifically decreased by immunodepletion. (A) Western blot analysis of immunodepleted extract. For immunodepletion, the salt concentration of splicing-competent nuclear extract was increased to $750 \mathrm{mM} \mathrm{KCl}$ and incubated with two sets of beads for $2 \mathrm{~h}$ each, followed by dialysis against RD100 buffer. A Smu1-specific antibody ( $\Delta$ Smu1) or no antibody (Mock) was coupled to the beads beforehand. To monitor bead-specific effects, a parallel extract was analysed that was not exposed to beads, but only to an increased salt concentration (SE750). Equal protein concentrations from each extract were loaded, as well as a ladder of the mock-depleted extract, to assess depletion efficiency. The extracts were separated by 4-12\% SDS-PAGE, transferred to a nitrocellulose membrane and analysed by immunoblotting using antibodies against Smu1 and RED as well as core splicing factors (116K, 110K and SF3a66) or other B-specific proteins (MFAP1, FBP21 and hPrp38) as controls. (B) Quantification of protein levels in the depleted extract relative to the mock extract.

\subsection{Spliceosome activation is slowed down in $\Delta$ Smu1/RED extracts}

In order to assess the role of Smu1/RED, I investigated the kinetics of in vitro splicing of the MINX-MS2 pre-mRNA in the absence or presence of Smu1/RED. This pre-mRNA comprises a 120-nt-long intron, flanked by two exons; it is therefore able to perform both splicing reactions. The co-transcribed MS2 tag of the pre-mRNA binds efficiently to an MS2-MBP fusion protein, which in turn binds to amylose beads. However, the affinity of the MBP (maltose binding protein) for amylose is lower than that for maltose. This makes possible the affinity purification of spliceosomes assembled in vitro for compositional analysis by gel electrophoresis or mass spectrometry. Analysis of MINX pre-mRNA splicing by denaturing gel electrophoresis revealed that both splicing steps occurred in the absence of Smu1/RED but less efficiently; this was manifested by the delayed appearance of both intermediates and products upon depletion (Fig. 3.4A).

After 60 minutes of splicing $\sim 85 \%$ of the MINX pre-mRNA had been converted into mature mRNA in mock depleted extract, while only $\sim 40 \%$ had been spliced in $\Delta$ Smu1/RED extract (Fig. 3.4C). Investigation of the spliceosome assembly on a native gel showed an increased B complex signal relative to the mock at all incubation times when Smu1/RED had been depleted (Fig. 3.4B). However, the signal decreased over time and the subsequently formed $\mathrm{B}^{\text {act }}$ and $\mathrm{C}$ complexes could be detected. 


\section{Results}

This suggests that the accumulation of B complexes was only transient and that the activation was impaired, but not blocked. In contrast no accumulation of A complexes was observed. Taken together, these results suggest that Smu1 and RED are not essential to form functional spliceosomes on the MINX pre-mRNA, but that their activation is delayed in the absence of the two proteins. Thus, Smu1 and RED appear to facilitate/accelerate the transition from the $B$ to the $B^{\text {act }}$ complex.

A

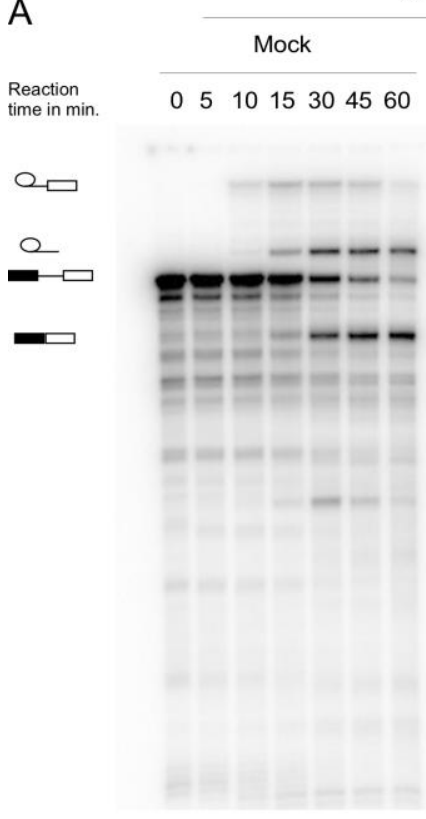

MINX

$\Delta$ Smu1/RED

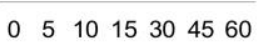

C

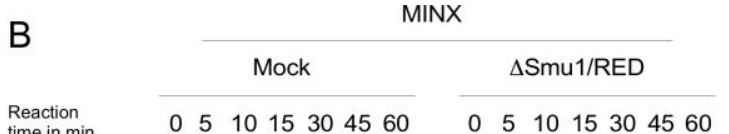

$0 \quad 51015304560$

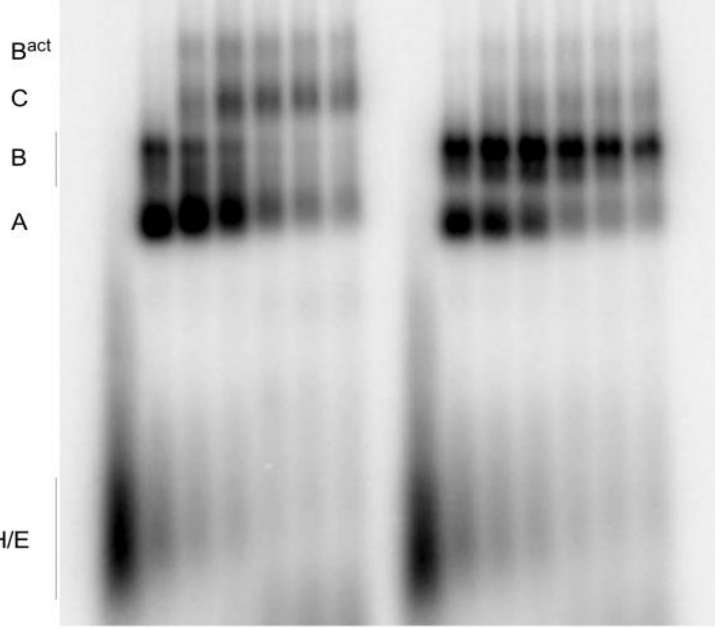

Splicing of MINX

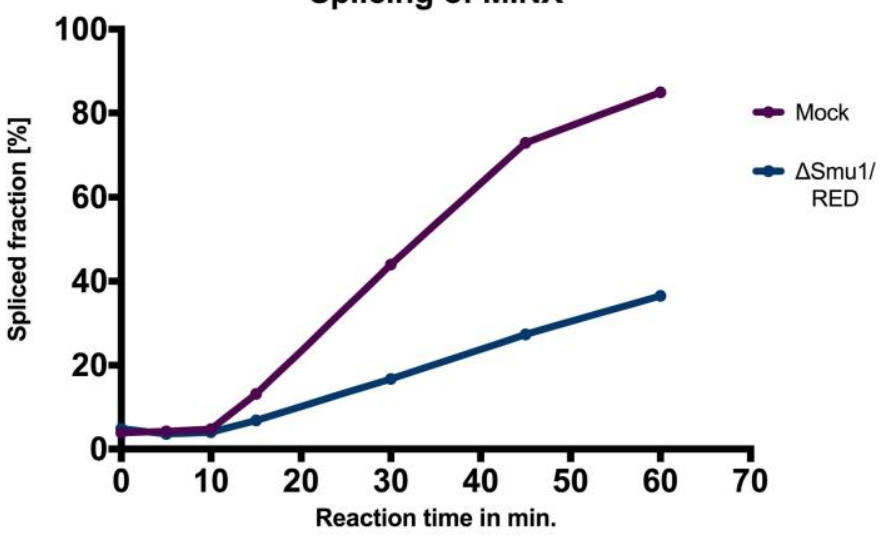

Figure 3.4. The B-to-Bact transition is slowed down in $\Delta$ Smu1/RED extract. Kinetics of in vitro splicing $(A)$ and spliceosome assembly (B) of MINX-120 pre-mRNA in the presence or absence of Smu1/RED. ${ }^{32} \mathrm{P}$-labelled pre-mRNA was incubated under splicing conditions in the presence of depleted or mock-depleted HeLa nuclear extract for the times indicated. (A) RNA was analysed by denaturing PAGE and visualized by autoradiography. The pre-mRNA, splicing intermediates and producs are indicated on the left. (B) Spliceosomal complex formation was analysed on a native agarose gel and visualized by autoradiography. The positions of the $\mathrm{H} / \mathrm{E}, \mathrm{A}, \mathrm{B}, \mathrm{C}$, and $\mathrm{B}^{\text {act }}$ complexes are indicated on the left. (C) Quantification of the kinetics of pre-mRNA to mRNA conversion over the reaction time. 


\subsection{Splicing efficiency is intron length dependent}

The RNAseq data indicated that the introns retained in cells upon Smu1/RED depletion had a pre-dominant length of $75-100 \mathrm{nt}$, while the splicing of longer introns was much less strongly affected. Therefore, I designed MINXMS2 constructs containing introns with $70,80,90$ or 150 nucleotides (MINX-70, $-80,-90$ and -150 ) by removing or adding sequences between the 5'SS and the branch site (BS) (Fig. 3.5A). Thereby, the 5'SS - BS distance was extended/reduced from $96 \mathrm{nt}$ to 126, 66, 56 and $46 \mathrm{nt}$, respectively, while the distance between the BS and the 3'SS remained unchanged. Importantly, the essential splicing motifs - including both splice sites, BS and polypyrimidine tract (PY tract) - remained unaltered.

In untreated nuclear extract, splicing of the MINX-150 pre-mRNA was as efficient as that of MINX-120. Both exhibited similar kinetics and most of both pre-mRNAs ( 75\%) had been spliced after 45 minutes (Fig. 3.5B and C). This indicates that elongation of the MINX intron by 30 nucleotides did not have a significant effect on splicing or spliceosome formation. When the intron was shortened to $90 \mathrm{nt}$, splicing-product formation occurred quite efficiently, although with somewhat altered kinetics. At each time point less of the splicing products had been formed, and 60 minutes were required to splice $\sim 75 \%$ of the pre-mRNA. Further shortening of the intron to $80 \mathrm{nt}$ revealed an even greater delay in splicing; after 60 minutes only $\sim 55 \%$ had been spliced. When the intron was truncated to $70 \mathrm{nt}$ barely any spliced product could be detected. The differences in splicing efficiency suggest that the productivity of the spliceosome depends on the intron length when this length falls below a certain critical value $(<120 \mathrm{nt})$.

Investigation of the spliceosome assembly on native gels showed that spliceosomal complexes were formed with similar efficiency on MINX-120 and MINX-150 (Fig. 3.5D). When spliceosomes were assembled on MINX-90 the $\mathrm{B}^{\text {act }}$ complex signal at later time points (30-60 min) appeared slightly stronger, while on MINX-80 the B complex was clearly increased after 10 minutes. This is consistent with the moderately reduced splicing efficiencies of the shortened pre-mRNAs which appears to be caused by an impaired spliceosome assembly.

With MINX-70 a strong accumulation of A complexes was obtained at all time points. While moderate signals for the $B$ complex could be observed, no $B^{\text {act }}$ or $C$ complexes were visible, indicating the $B$ complex formation is challenging on such a short intron. Taken together, the sequential shortening of the MINX intron resulted in increasingly impaired spliceosome assembly and splicing, suggesting that both depend on the intron length and can be completely inhibited when it falls below a critical size of $\sim 70 \mathrm{nt}$. 


\section{Results}

\section{A}
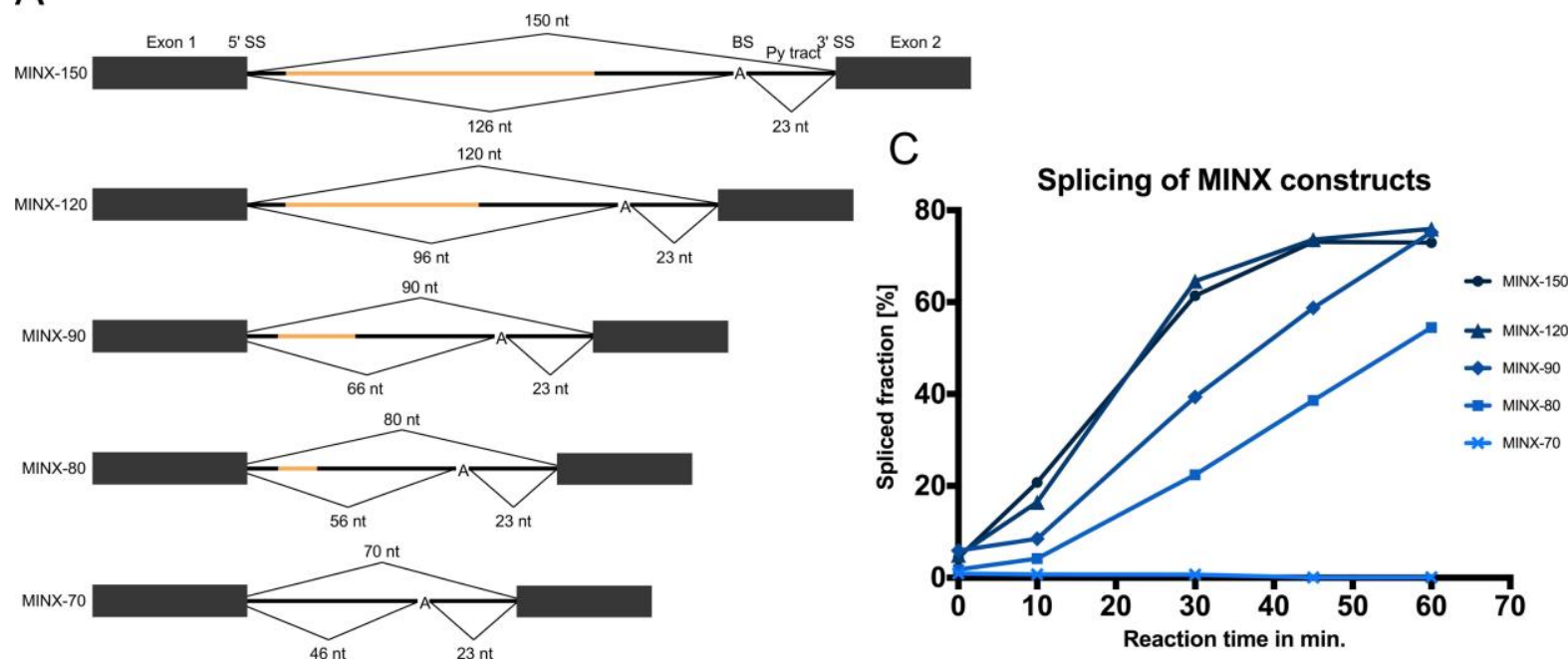

B

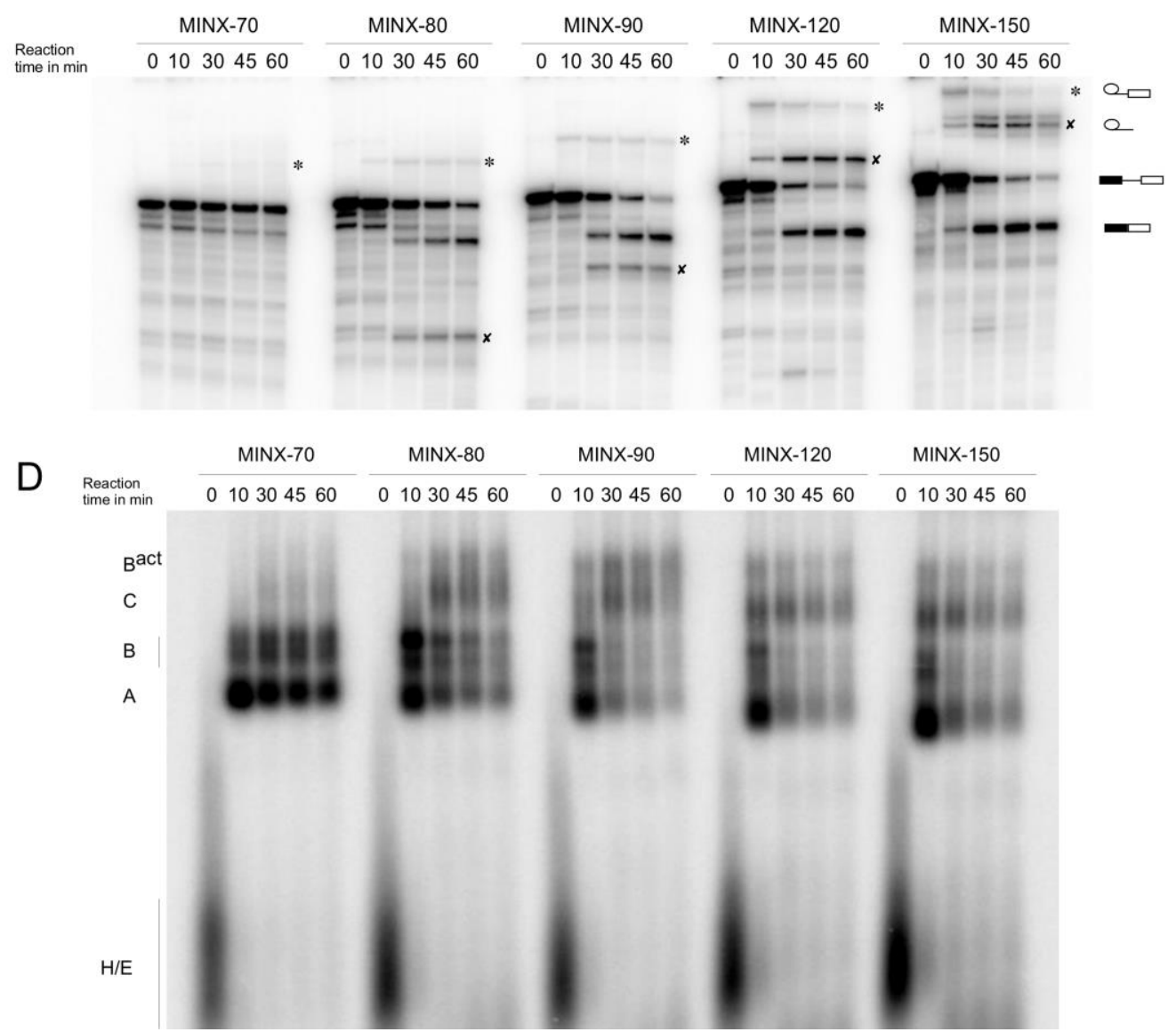

Figure 3.5. Splicing efficiency and spliceosome assembly are length-dependent. (A) Schematic of MINX-MS2 constructs with varying intron lengths. The MINX-120 intron (120 nt) was extended by $30 \mathrm{nt}$ (MINX-150) or truncated by $30 \mathrm{nt}$ (MINX-90), $40 \mathrm{nt}$ (MINX-80) or 50 nt (MINX-70). Reactive sites (5'SS, BP, 3'SS) and the polypyrimidine (PY) tract remained unchanged. Kinetics of in vitro splicing (B) and spliceosome assembly (D) of the distinct MINX pre-mRNA constructs. ${ }^{32} \mathrm{P}$-labelled pre-mRNAs were incubated under splicing conditions in the presence of regular HeLa extract for the indicated times. (B) RNA was analysed by denaturing PAGE and visualized by autoradiography. The pre-mRNA and splicing intermediates and products are indicated on the right. " $*$ " and " $x$ " mark the intron-3'exon intermediate or spliced-out intron of the distinct pre-mRNA constructs, which migrate differently owing to their different sizes. (C) Quantification of the kinetics of the conversion of pre-mRNA to mRNA over the reaction time. (D) Spliceosomal complex formation was analysed on a native agarose gel and visualized by autoradiography. The positions of the $H / E, A, B, C$, and $B^{\text {act }}$ complexes are indicated on the left. 
Results

\subsection{Smu1 and RED are essential for in vitro splicing and spliceosome assembly when}

\section{introns are short}
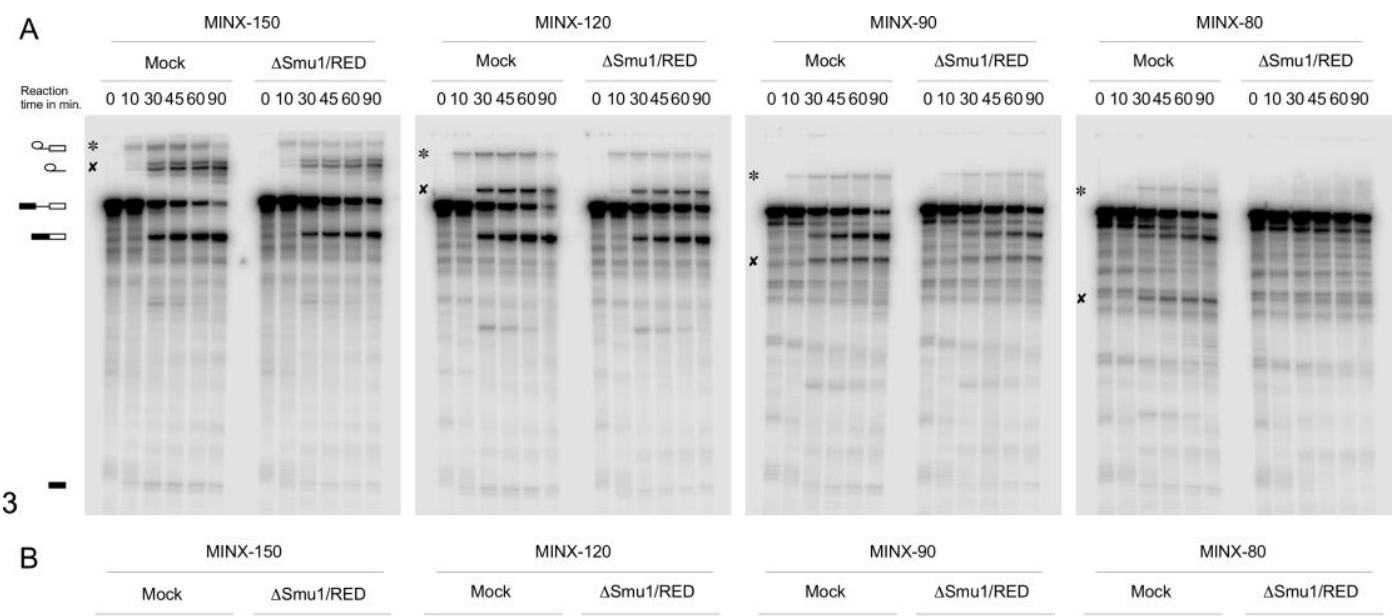

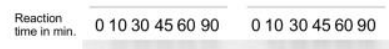
$01030456090 \quad 01030456090$
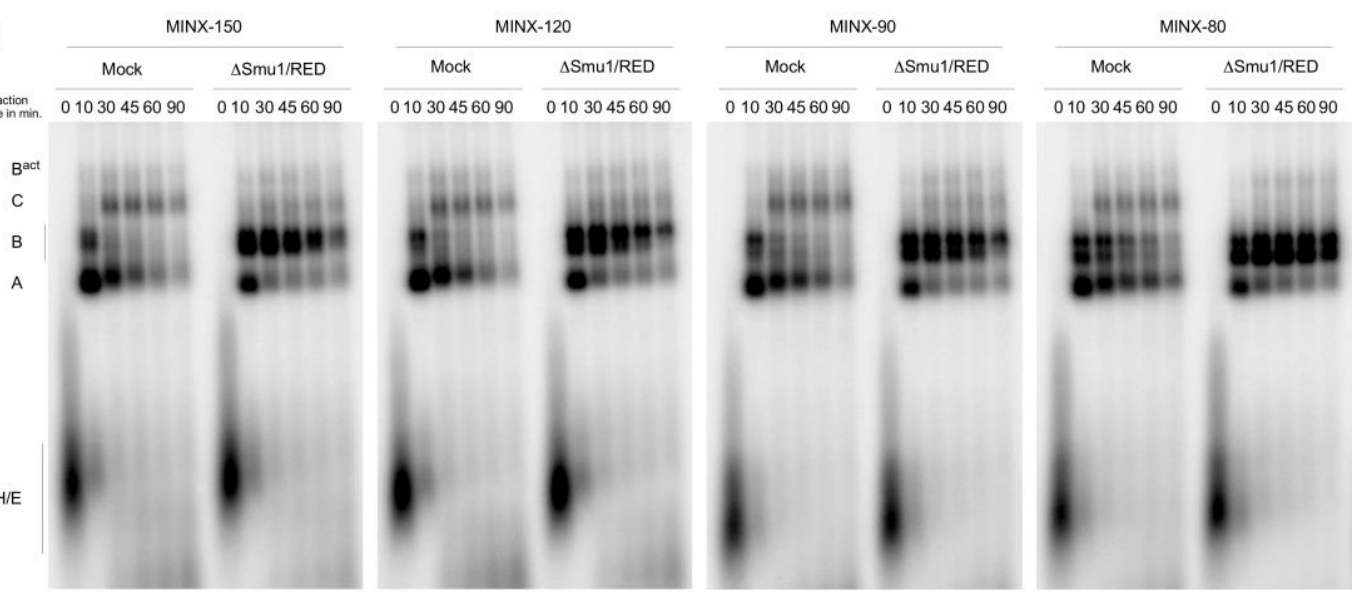

$01030456090 \quad 01030456090$

C

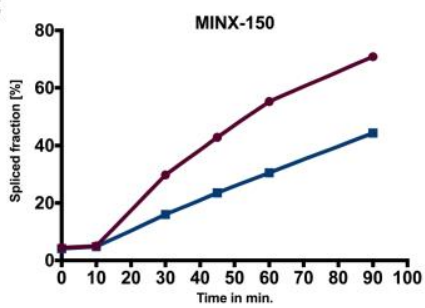

$\mathrm{E}$

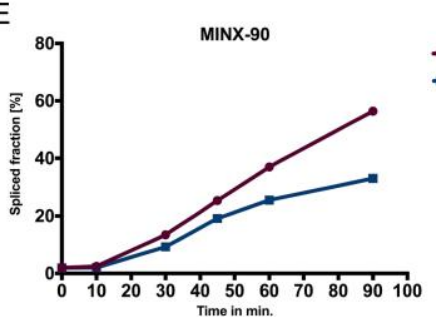

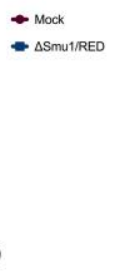

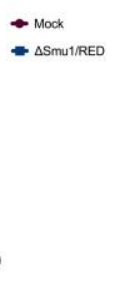

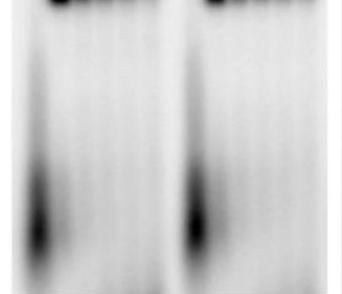

D

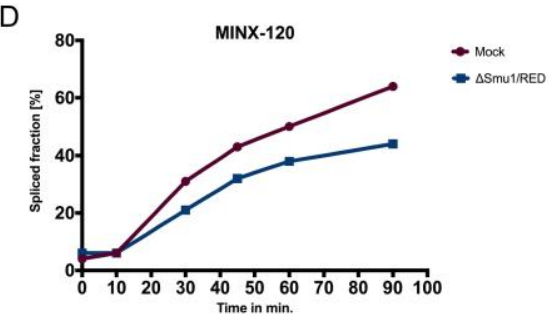

$\mathrm{F}$

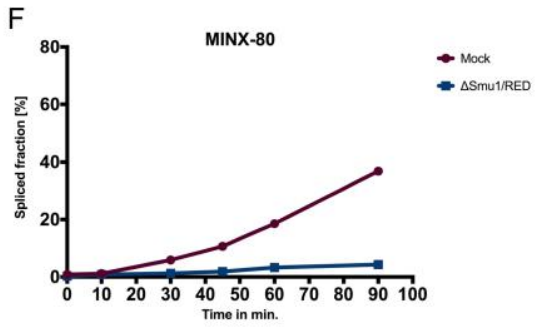

Figure 3.6. Splicing and spliceosome assembly are slowed down/inhibited by the Smu1/RED depletion. Kinetics of splicing (A) and spliceosome assembly (B) in vitro of MINX pre-mRNA constructs in the presence or absence of Smu1/RED. ${ }^{32}$ P-labelled pre-mRNA was incubated under splicing conditions in the presence of depleted or mock-depleted HeLa nuclear extract for the times indicated. (A) RNA was analysed by denaturing PAGE and visualized by autoradiography. The pre-mRNA and splicing intermediates and products are indicated on the right. "*" and " $x$ " mark respectively the intron-3'exon intermediate and the intron of the distinct pre-mRNA constructs, which migrate differently owing to their different sizes. (B) Spliceosomal complex formation was analysed on a native agarose gel and visualized by autoradiography. The positions of the H/E, A, B, C, and $B^{\text {act }}$ complexes are indicated on the left. (C-F) Quantification of premRNA to mRNA conversion in $\triangle$ Smu1/RED extract relative to the mock-depleted sample. 


\section{Results}

As a next step, I investigated the effect of the Smu1/RED depletion on the splicing of the four functional MINX constructs (MINX-80, $-90,-120$ and -150) in the Smu1/RED-depleted extract. To that end, each pre-mRNA was incubated with Smu1/RED-depleted or mock-depleted extract and spliceosome assembly or splicing-product formation was analysed on native or denaturating gels, respectively. In the absence of Smu1/RED, MINX-150 and MINX-120 were spliced $\sim 30 \%$ less efficiently (Fig. 3.6A, C and D) than in the mock-depleted extract, and in contrast to the mock control there was a strong accumulation of B complexes at all time points with $B$ complexes still detectable after 90 minutes (Fig. 3.6B). Splicing of MINX-90 in $\Delta$ Smu1/RED extract was $50 \%$ less efficient than in the mock-depleted extract (Fig. 3.6E) and slightly more B complexes accumulated. Thus, MINX-90 appears to be moderately more affected by the depletion than MINX-150 and MINX-120. Splicing of MINX-80, however, was drastically reduced in the absence of Smu1/RED, with the pre-mRNA-to-mRNA conversion reduced by $90 \%$ (Fig. 3.6F). Consistent with this, a significantly increased B complex signal relative to the mock extract could be detected on the native gel, while no signals corresponding to later $\mathrm{B}^{\text {act }}$ or $\mathrm{C}$ spliceosomal complexes were visible, suggesting a severe impairment of the B-to-Bact transition. Taken together, these results indicate that Smu1 and RED function to facilitate spliceosome assembly on longer introns and become essential factors for the activation of spliceosomes assembling on short introns with a length of $\sim 80 \mathrm{nt}$.

\subsection{Splicing of MINX-80 can be restored by addition of Smu1/RED}

In vitro splicing experiments with Smu1/RED-depleted extract suggested that these two proteins are involved in the activation of spliceosomal B complexes. To exclude the possibility that the observed effect was caused by codepletion of additional splicing factors, I performed a complementation experiment with human Smu1 and RED proteins, obtained by co-expression in insect cells. For that purpose, RED was equipped with an $\mathrm{N}$-terminal His tag and Smu1 with a Strep-II tag, and for both proteins baculoviral DNA was generated in E. coli DH10MultiBacY cells. The viral DNA was transfected into Sf9 insect cells for reconstitution of infectious virus particles. For protein expression, optimised High Five insect cells were transfected with the virus particles, and after 60-72 hours, the dimer could be purified from the cells by nickel-column chromatography (Fig 3.7A). The purified protein was incubated with HeLa nuclear extract before initiating the splicing reaction, to allow the formation of potentially important interactions. Since splicing of MINX-80 was most strongly affected by the Smu1/RED-depletion, it was used as substrate in the following experiments, except where stated otherwise.

As shown in Fig. 3.7B, complementation of the Smu1/RED-depleted reaction with the purified dimer completely resolved the assembly blockade and allowed the formation of $\mathrm{B}^{\text {act }}$ and $\mathrm{C}$ complexes. Furthermore, splicing of MINX80 was restored to levels comparable to that observed with mock-depleted extract (Fig. 3.7C and D). This indicates that indeed no splicing factors other than Smu1/RED were responsible for stalling the spliceosome assembly. 


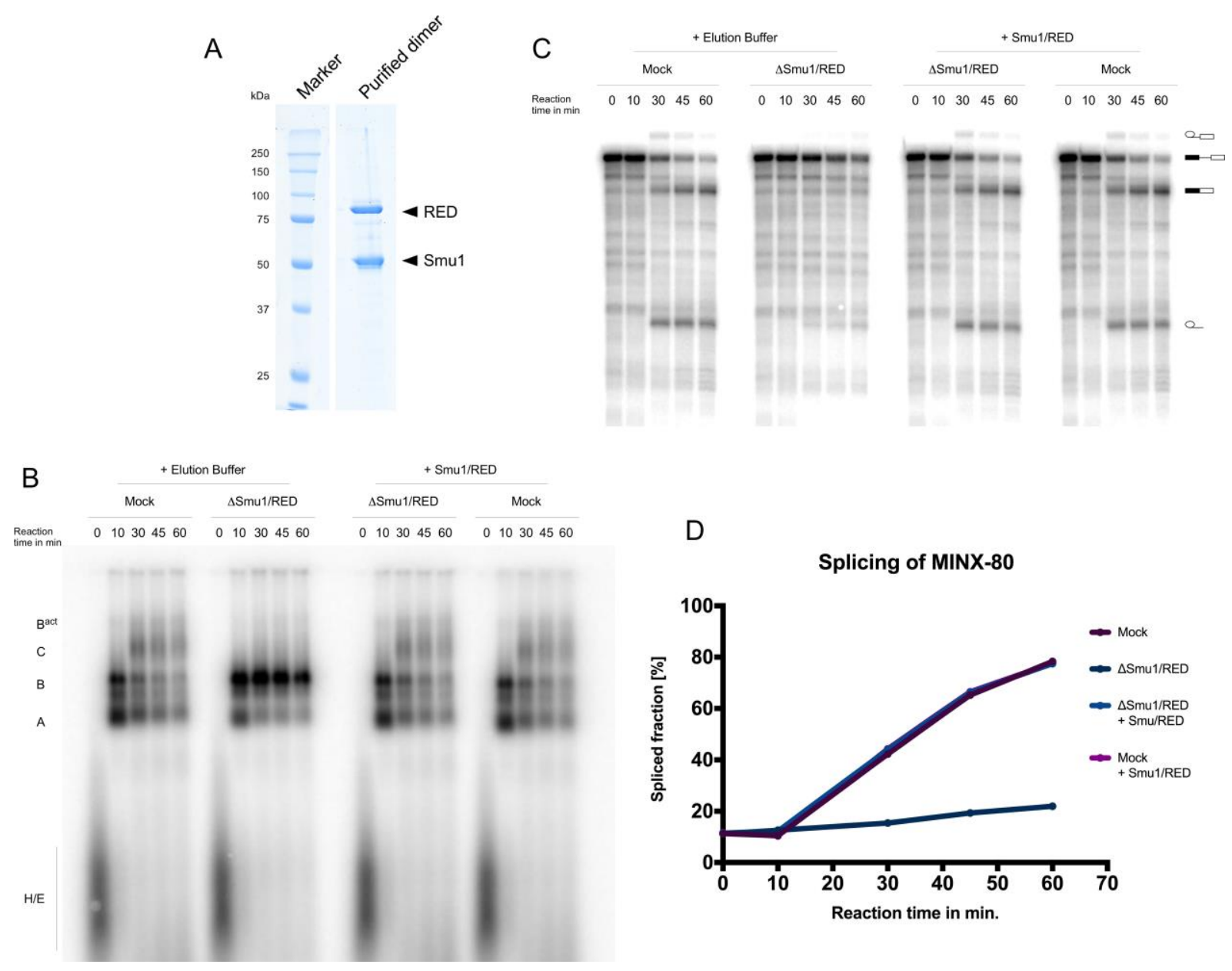

Figure 3.7. Purified recombinant Smu1/RED is sufficient to restore spliceosome activation and splicing. (A) Human Smu1 and RED, expressed and purified from High Five insect cells were separated by SDS-PAGE and stained with Coomassie. Kinetics of spliceosome assembly (B) and in vitro splicing (C) after complementation with the Smu1/RED dimer obtained from co-expression in insect cells. The purified proteins, or only High Five Elution buffer, were pre-incubated with depleted or mock-depleted extract and then with the ${ }^{32}$ P-labelled MINX-80 pre-mRNA under splicing conditions for the times indicated. (B) Spliceosomal complex formation was analysed on a native agarose gel and visualized by autoradiography. The positions of the $\mathrm{H} / \mathrm{E}, \mathrm{A}, \mathrm{B}, \mathrm{C}$, and $\mathrm{B}^{\text {act }}$ complexes are indicated on the left. (C) RNA was analysed by denaturing PAGE and visualized by autoradiography. The pre-mRNA and splicing intermediates and products are indicated on the right. (D) Quantification of the kinetics of pre-mRNA-to-mRNA conversion over the reaction time.

\subsection{Smu1/RED immunodepletion does not affect the recruitment of additional spliceosomal factors to the B complex}

Complementation of depleted extract with the purified Smu1/RED dimer restored splicing of the MINX-80 pre-mRNA to mock-depletion levels, indicating that the observed defects in splicing and spliceosome assembly were not due to co-depletion of other spliceosomal factors required for the activation. Previously, it has been proposed that Smu1 and RED might serve as a binding platform (Ulrich et al., 2016) for other spliceosomal proteins. 


\section{Results}

To investigate whether Smu1 and RED recruit spliceosomal factors that are required for a functional activation, or promote the activation themselves, I assembled mock (i.e wildtype) and $\Delta$ Smu1/RED-stalled B complexes on MINX80 , separated the particles by glycerol-gradient ultracentrifugation, performed MS2 affinity selection and compared the snRNA and protein composition of the affinity-purified complexes. The mass spectrometry analysis was performed by the laboratory of Henning Urlaub (MPI-bpc, Göttingen).

The gradient profile is illustrated in Fig. 3.8A. It shows that the B complexes assembled in mock-depleted extract after $8 \mathrm{~min}$ of in vitro splicing, peaked in the fractions 14-15, while $\Delta$ Smu1/RED B complexes (after incubation for $8 \mathrm{~min}$ ) peaked in fraction 12-14, and thus migrated one or two fractions slower. This distinct migration might suggest that the stalled complexes are smaller than the B complexes formed after 8 min in the mock extract, or that they may have adopted a more relaxed conformation, or possibly both. However, the snRNA composition of $\triangle$ Smu1/RED B complexes (Fig. 3.8B) was identical to that of the mock B complexes, comprising the characteristic U2, U4, U5 and U6 snRNAs. The absence of the U1 snRNA suggests that the Prp28-mediated destabilization of the U1 snRNP was not affected by the depletion of Smu1/RED, while the presence of the U4 snRNA indicates that the Brr2mediated unwinding of the U4/U6 helix had not yet occurred. Thus, depletion of Smu1/RED stalled spliceosome assembly after the U1 snRNP release and before the unwinding of the U4/U6 duplex by Brr2.

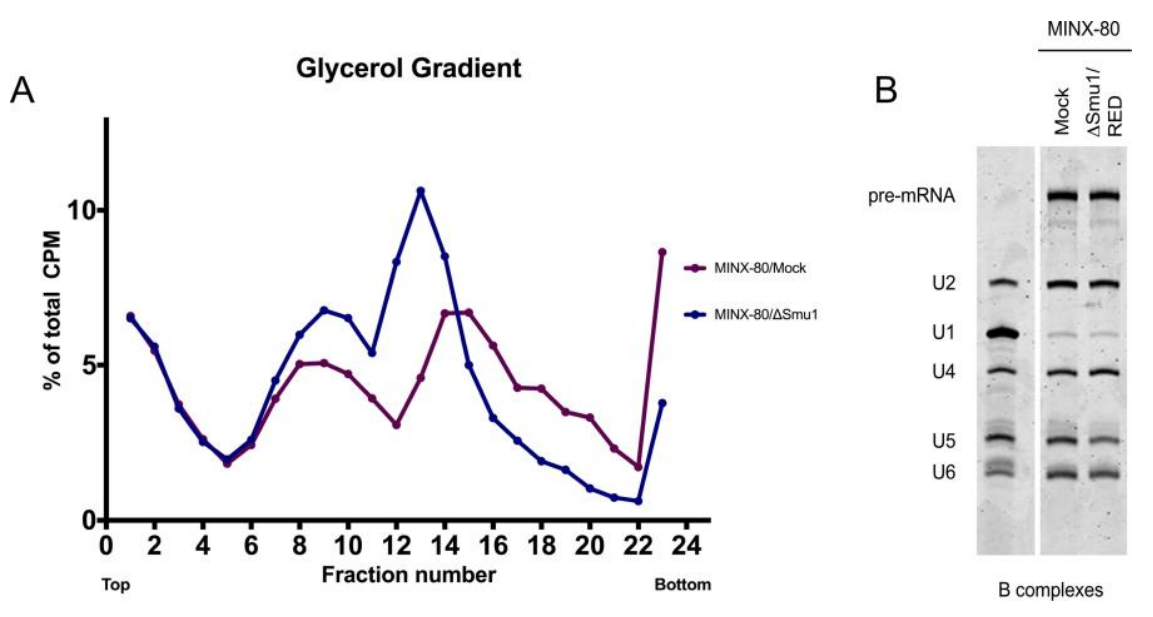

Figure 3.8. Depletion of Smu1/RED stalls spliceosome assembly at the B complex level. (A) The glycerol-gradient sedimentation profile of B complexes assembled on MINX-80 in the presence or absence of Smu1/RED. B complexes were assembled for 8 min under splicing conditions in the presence of depleted or mock-depleted HeLa nuclear extract. The migration of the ${ }^{32} \mathrm{P}$-pre-mRNA was determined by Cherenkov counting. (B) snRNA composition of the spliceosomal complexes. The spliceosomal complexes in the peak fractions ( $\triangle$ Smu1/RED B complex, fractions 12-14; mock B complex, fractions 14-16) were subjected to MS2 affinity purification and the RNA extracted from the purified complexes was analysed by denaturing PAGE followed by staining with SYBR Gold. 
Table 3.1: Protein compositions of the $\triangle$ Smu1/RED and mock (kinetically stalled) B complexes. Proteins of the affinity-purified complexes indicated were identified by LC-MS/MS after separation by 4-12 \% SDS-PAGE. The number shown is the total number of peptides sequenced for the indicated protein. Proteins are grouped according to function or association. Proteins considered as common contaminants, such as ribosomal proteins and non-reproducibly detected proteins are not shown.

\begin{tabular}{|c|c|c|c|c|}
\hline \multirow[b]{2}{*}{ Protein } & \multirow[b]{2}{*}{ hGene Name } & \multirow[b]{2}{*}{$\mathrm{kDa}$} & \multicolumn{2}{|c|}{ MINX-80 } \\
\hline & & & Mock & $\Delta$ Smu1/RED \\
\hline \multicolumn{5}{|l|}{ Sm proteins } \\
\hline B & SNRPB & 24 & 45 & 48 \\
\hline D1 & SNRPD1 & 13 & 19 & 20 \\
\hline D2 & SNRPD2 & 14 & 30 & 41 \\
\hline D3 & SNRPD3 & 14 & 46 & 62 \\
\hline $\mathrm{E}$ & SNRPE & 11 & 26 & 26 \\
\hline $\mathrm{F}$ & SNRPF & 10 & 5 & 8 \\
\hline G & SNRPG & 8 & 7 & 10 \\
\hline \multicolumn{5}{|l|}{ U1 snRNP } \\
\hline U1-70K & $\begin{array}{l}\text { SNRNP70 } \\
\end{array}$ & 52 & 11 & 22 \\
\hline U1-A & SNRPA & 31 & 11 & 13 \\
\hline U1-C & SNRPC & 17 & 1 & 2 \\
\hline \multicolumn{5}{|l|}{$17 \mathrm{~S}$ U2 snRNP } \\
\hline $\mathrm{U}^{\prime} \mathrm{A}^{\prime}$ & $\begin{array}{l}\text { SNRNPA1 } \\
\end{array}$ & 28 & 75 & 73 \\
\hline U2B" & SNRPB2 & 25 & 20 & 26 \\
\hline SF3a120 & SF3A1 & 89 & 159 & 161 \\
\hline SF3a66 & SF3A2 & 51 & 25 & 26 \\
\hline SF $3 a 60$ & SF3A3 & 59 & & \\
\hline SF3b155 & SF3B1 & 146 & 295 & 323 \\
\hline SF3b145 & SF3B2 & 100 & 144 & 181 \\
\hline SF $3 b 130$ & SF3B3 & 136 & 337 & 389 \\
\hline SF3b49 & SF3B4 & 44 & & \\
\hline SF3b14a/p14 & SF3B14 & 15 & 18 & 22 \\
\hline SF3b10 & SF3b5 & 10 & 5 & 7 \\
\hline \multicolumn{5}{|l|}{$17 \mathrm{~S}$ U2 related } \\
\hline hPRP43 & \begin{tabular}{|l} 
DHX15 \\
\end{tabular} & 91 & 45 & 36 \\
\hline U2AF65 & U2AF2 & 54 & 28 & 48 \\
\hline SPF45 & \begin{tabular}{|l} 
RBM17 \\
\end{tabular} & 45 & 16 & 5 \\
\hline SR140 (fSAPa) & U2SURP & 118 & 23 & 51 \\
\hline CHERP & \begin{tabular}{|l} 
CHERP \\
\end{tabular} & 104 & 12 & 15 \\
\hline SF3b125 & \begin{tabular}{|l} 
DDX42 \\
\end{tabular} & 103 & 8 & 2 \\
\hline U2AF35 & \begin{tabular}{|l} 
U2AF1 \\
\end{tabular} & 28 & 11 & 19 \\
\hline SPF31 & DNAJC8 & 30 & 1 & 7 \\
\hline hPRP5 & \begin{tabular}{|l} 
DDX46 \\
\end{tabular} & 117 & 5 & 25 \\
\hline PUF60 & \begin{tabular}{|l} 
PUF60 \\
\end{tabular} & 60 & 16 & 27 \\
\hline SPF30 & SMNDC1 & 27 & 11 & 21 \\
\hline
\end{tabular}

\begin{tabular}{|l|l|c|c|c|}
\hline U5 & \multicolumn{5}{l|}{} \\
\cline { 2 - 5 } $220 \mathrm{~K}$ & PRPF8 & $\mathbf{2 7 4}$ & 658 & 647 \\
\cline { 2 - 5 } $116 \mathrm{~K}$ & SNRNP200 & $\mathbf{2 4 5}$ & 803 & 754 \\
\hline $40 \mathrm{~K}$ & EFTUD2 & $\mathbf{1 0 9}$ & 343 & 344 \\
\hline $102 \mathrm{~K}$ & WDR57 & $\mathbf{3 9}$ & 95 & 80 \\
\hline $15 \mathrm{~K}$ & PRPF6 & $\mathbf{1 0 7}$ & 271 & 254 \\
\hline $100 \mathrm{~K}$ & TXNL4A & $\mathbf{1 7}$ & 13 & 17 \\
\hline $52 \mathrm{~K}$ & DDX23 & $\mathbf{9 6}$ & 127 & 116 \\
\cline { 2 - 5 } & CD2BP2 & $\mathbf{3 8}$ & 31 & 10 \\
\hline
\end{tabular}

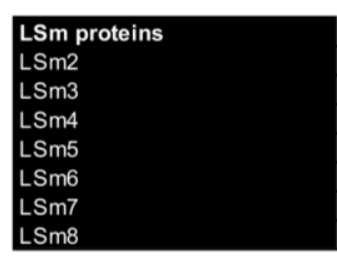

\begin{tabular}{|l|c|c|c|}
\hline LSM2 & $\mathbf{1 1}$ & 15 & 15 \\
\hline LSM3 & $\mathbf{1 2}$ & 3 & 7 \\
\hline LSM4 & $\mathbf{1 5}$ & 5 & 9 \\
\hline LSM5 & $\mathbf{1 0}$ & & \\
\hline LSM6 & $\mathbf{9}$ & 3 & 7 \\
\hline LSM7 & $\mathbf{1 2}$ & 4 & 8 \\
\hline LSM8 & $\mathbf{1 0}$ & 13 & 14 \\
\hline
\end{tabular}

\begin{tabular}{|c|c|c|c|c|}
\hline \multirow{2}{*}{\begin{tabular}{|l|} 
U4/U6 \\
$90 \mathrm{~K}$
\end{tabular}} & \multirow[b]{2}{*}{\begin{tabular}{|l} 
PRPF3 \\
\end{tabular}} & \multirow[b]{2}{*}{78} & \multirow[b]{2}{*}{111} & \multirow[b]{2}{*}{158} \\
\hline & & & & \\
\hline $60 \mathrm{~K}$ & PRPF4 & 58 & 108 & 124 \\
\hline $20 \mathrm{~K}$ & $\mathrm{PPIH}$ & 19 & 19 & 32 \\
\hline $61 \mathrm{~K}$ & PRPF31 & 55 & 75 & 88 \\
\hline $15.5 \mathrm{~K}$ & NHP2L1 & 14 & 7 & 14 \\
\hline \multicolumn{5}{|l|}{ U4/U6.U5 } \\
\hline $110 \mathrm{~K}$ & SART1 & 90 & 278 & 350 \\
\hline $65 K$ & USP39 & 65 & 59 & 46 \\
\hline $27 K$ & & 27 & & \\
\hline RBM42 & RBM42 & 48 & & \\
\hline
\end{tabular}




\section{Results}

\begin{tabular}{|c|c|c|c|c|}
\hline \multirow[b]{2}{*}{ Protein } & \multirow[b]{2}{*}{ hGene Name } & \multirow[b]{2}{*}{ kDa } & \multicolumn{2}{|c|}{ MINX-80 } \\
\hline & & & Mock & $\Delta \mathrm{Smu} 1 / \mathrm{RED}$ \\
\hline \multicolumn{5}{|l|}{ A complex } \\
\hline THRAP3 & THRAP3 & 109 & 23 & 22 \\
\hline CCAR1 & CCAR1 & 133 & 2 & 6 \\
\hline RBM5 & RBM5 & 92 & 6 & 7 \\
\hline S164 (fSAP94) & RBM25 & 100 & 6 & 15 \\
\hline RBM10 & RBM10 & 104 & & \\
\hline SF1 & SF1 & 68 & & \\
\hline FBP11 & PRPF40A & 109 & 4 & 9 \\
\hline SF4 & SUGP1 & 72 & 2 & 12 \\
\hline \multirow{2}{*}{$\begin{array}{l}\text { Pre-B complex } \\
\text { hPRP4-Kinase }\end{array}$} & & & & \\
\hline & PRPF4B & 115 & 72 & 62 \\
\hline
\end{tabular}

\begin{tabular}{|c|c|c|c|c|}
\hline \multirow{2}{*}{$\begin{array}{l}\text { B complex } \\
\text { hPRP38 }\end{array}$} & \multirow[b]{2}{*}{ PRPF38A } & \multirow[b]{2}{*}{37} & \multirow[b]{2}{*}{45} & \multirow[b]{2}{*}{45} \\
\hline & & & & \\
\hline hSnu23 & ZMAT2 & 24 & 12 & 16 \\
\hline MFAP1 & MFAP1 & 52 & 30 & 35 \\
\hline RED & IK & 66 & 83 & 4 \\
\hline hSmu-1 (fSAP57) & SMU1 & 58 & 87 & 3 \\
\hline FBP21 & WBP4 & 43 & 13 & 29 \\
\hline Npw38BP & WBP11 & 70 & 7 & 0 \\
\hline Npw38 & PQBP1 & 30 & 2 & 1 \\
\hline UBL5 & UBL5 & 9 & 5 & 4 \\
\hline
\end{tabular}

\begin{tabular}{|c|c|c|c|c|}
\hline \multirow{2}{*}{$\begin{array}{l}\text { hPRP19/CDC5L } \\
\text { hPRP19 }\end{array}$} & \multirow[b]{2}{*}{ PRPF19 } & \multirow{2}{*}{55} & \multirow[b]{2}{*}{85} & \multirow[b]{2}{*}{40} \\
\hline & & & & \\
\hline CDC5L & & 92 & 95 & 43 \\
\hline SPF27 & BCAS2 & 26 & 13 & 2 \\
\hline PRL1 & PLRG1 & 57 & 46 & 21 \\
\hline CCAP1 & HSPA8 & 71 & 156 & 139 \\
\hline CCAP2 & CWC15 & 27 & 6 & 2 \\
\hline catenin, b-like 1 & CTNNBL1 & 62 & 20 & 5 \\
\hline
\end{tabular}

\begin{tabular}{|l|l|c|c|c|}
\hline $\begin{array}{l}\text { CRNKL1 (hSYF3) } \\
\text { SKIP }\end{array}$ & CRNKL1 & $\mathbf{9 9}$ & $\mathbf{7 6}$ & 31 \\
\cline { 2 - 5 } RBM22 & SNW1 & $\mathbf{6 1}$ & 60 & 22 \\
\cline { 2 - 5 } PPlase-like 1 (PPIL1) & RBM22 & $\mathbf{4 7}$ & 15 & 2 \\
\cline { 2 - 5 } & PPIL1 & $\mathbf{1 8}$ & 6 & 2 \\
\cline { 2 - 5 } G10 (fSAP17) & BUD31 & $\mathbf{1 7}$ & 11 & 4 \\
\hline
\end{tabular}

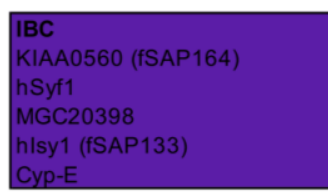

\begin{tabular}{|l|c|c|c|}
\hline AQR & $\mathbf{1 7 1}$ & 72 & 35 \\
\hline XAB2 & $\mathbf{1 0 0}$ & 51 & 27 \\
\hline ZNF830 & $\mathbf{4 2}$ & 12 & 0 \\
\hline ISY1 & $\mathbf{3 3}$ & 13 & 7 \\
\hline PPIE & $\mathbf{3 3}$ & 18 & 5 \\
\hline
\end{tabular}

$B^{\text {act }}$ complex

KIAA1604 (fSAPb)

hPRP17

NY-CO-10

RNF113A

PPIL2 (Сур-60)

PRCC

MGC23918

SRm160

SRm300

PPlase-like 3b

\begin{tabular}{|l|c|c|c|}
\hline CWC22 & $\mathbf{1 0 6}$ & 35 & 1 \\
\hline PRPF17/CDC4 & $\mathbf{6 6}$ & 27 & 12 \\
\hline CWC27 & $\mathbf{5 4}$ & 10 & 2 \\
\hline RNF113A & $\mathbf{3 9}$ & & \\
\hline PPIL2 & $\mathbf{6 0}$ & 28 & 5 \\
\hline PRCC & $\mathbf{5 2}$ & 12 & 2 \\
\hline CCDC12 & $\mathbf{2 1}$ & 8 & 5 \\
\hline SRRM1 & $\mathbf{1 0 3}$ & 16 & 3 \\
\hline SRRM2 & $\mathbf{3 0 0}$ & 71 & 48 \\
\hline PPIL3 & $\mathbf{1 9}$ & & \\
\hline
\end{tabular}

1st step factors

hPRP2

GPKOW

CCDC49

\begin{tabular}{|l|c|c|c|}
\hline DHX16 & $\mathbf{1 1 9}$ & 19 & 7 \\
\hline GPKOW & $\mathbf{5 2}$ & 4 & 1 \\
\hline CWC25 & $\mathbf{5 0}$ & & \\
\hline
\end{tabular}

C complex

GCIP p29 (fSAP29)

C9orf78 (HSPC220)

WDR70

NOSIP

PPWD1 (KIAA0073, CyP64)

PPIL4

CDK10

PPIG (SRcyp)

\begin{tabular}{|l|c|c|c|}
\hline SYF2 & $\mathbf{2 9}$ & 3 & 4 \\
\hline C9orf78 & $\mathbf{3 4}$ & & \\
\hline WDR70 & $\mathbf{3 3}$ & & \\
\hline NOSIP & $\mathbf{3 3}$ & & \\
\hline PPWD1 & $\mathbf{7 4}$ & & \\
\hline PPIL4 & $\mathbf{3 5}$ & & \\
\hline CDK10 & $\mathbf{3 5}$ & & \\
\hline PPIG & $\mathbf{8 9}$ & 2 & 0 \\
\hline
\end{tabular}

2nd step factors

hPRP22

hPRP18

hSlu7

hPRP16

\begin{tabular}{|l|c|c|c|}
\hline DHX8 & 139 & 8 & 2 \\
\hline PRPF18 & $\mathbf{4 0}$ & & \\
\hline SLU7 & $\mathbf{6 8}$ & 0 & 2 \\
\hline DHX38 & $\mathbf{1 4 0}$ & 26 & 14 \\
\hline
\end{tabular}


Results

\begin{tabular}{|c|c|c|c|c|}
\hline \multirow[b]{2}{*}{ Protein } & \multirow[b]{2}{*}{ hGene Name } & \multirow[b]{2}{*}{$\mathrm{kDa}$} & \multicolumn{2}{|c|}{ MINX-80 } \\
\hline & & & Mock & $\Delta$ Smu1/RED \\
\hline \multicolumn{5}{|l|}{ Putative $C^{*}$ proteins } \\
\hline \multirow{5}{*}{$\begin{array}{l}\text { DDX35 } \\
\text { Abstrakt } \\
\text { C19orf29 (NY-REN-24, cactin) } \\
\text { CXorf56 (FLJ22965) } \\
\text { FAM50A (HXC-26, XAP5) }\end{array}$} & DHX35 & 79 & 7 & 4 \\
\hline & DDX41 & 70 & 4 & 8 \\
\hline & CACTIN & 89 & & \\
\hline & CXorf56 & 25,6 & & \\
\hline & FAM50A & 40 & & \\
\hline
\end{tabular}

\begin{tabular}{|c|c|c|c|c|}
\hline \multirow{2}{*}{$\begin{array}{l}\text { SR proteins } \\
\text { SF2/ASF }\end{array}$} & \multirow[b]{2}{*}{ SRSF1 } & \multirow[b]{2}{*}{28} & \multirow[b]{2}{*}{37} & \multirow[b]{2}{*}{42} \\
\hline & & & & \\
\hline Sc35 & \begin{tabular}{|l} 
SRSF2 \\
\end{tabular} & 25 & & \\
\hline SRp20 & \begin{tabular}{|l|} 
SRSF3 \\
\end{tabular} & 19 & 10 & 12 \\
\hline SRp75 & SRSF4 & 57 & 12 & 12 \\
\hline SRp40 & \begin{tabular}{|l} 
SRSF5 \\
\end{tabular} & 31 & & \\
\hline SRp55 & \begin{tabular}{|l|} 
SRSF6 \\
\end{tabular} & 40 & 16 & 16 \\
\hline $9 G 8$ & \begin{tabular}{|l|} 
SRSF7 \\
\end{tabular} & 27 & 22 & 22 \\
\hline SRp46 & SRSF8 & 32 & & \\
\hline SRp30c & SRSF9 & 26 & 8 & 8 \\
\hline SRp38 & \begin{tabular}{|l|} 
SRSF10 \\
\end{tabular} & 31 & 7 & 3 \\
\hline SRSF11 & SRSF11 & 54 & 0 & 4 \\
\hline hTra-2 alpha & TRA2A & 33 & & \\
\hline hTra-2 beta (SFRS10) & TRA2B & 34 & & \\
\hline
\end{tabular}

\begin{tabular}{|c|c|c|c|c|}
\hline \multirow{2}{*}{$\begin{array}{l}\text { hnRNP } \\
\text { hnRNP A0 }\end{array}$} & \multirow{2}{*}{\begin{tabular}{|l|} 
HNRNPAO \\
\end{tabular}} & \multirow[b]{2}{*}{31} & \multirow[b]{2}{*}{10} & \multirow[b]{2}{*}{12} \\
\hline & & & & \\
\hline hnRNP A1 & HNRNPA1 & 39 & 44 & 67 \\
\hline hnRNP A3 & HNRNPA3 & 40 & 17 & 21 \\
\hline$h n R N P A B$ & HNRNPAB & 35 & 11 & 15 \\
\hline hnRNP A2/B1 & HNRNPA2B1 & 37 & 53 & 80 \\
\hline hnRNP C & HNRNPC & 33 & 17 & 17 \\
\hline hnRNP D & HNRNPDO & 24 & & \\
\hline hnRNP DL & \begin{tabular}{|l} 
HNRNPDL \\
\end{tabular} & 46 & 8 & 6 \\
\hline hnRNP F & HNRNPF & 46 & 6 & 2 \\
\hline hnRNP H1 & \begin{tabular}{|l} 
HNRNPH1 \\
\end{tabular} & 49 & 9 & 2 \\
\hline hnRNP H3 & HNRNPH3 & 37 & 10 & 6 \\
\hline hnRNP K & HNRNPK & 51 & 25 & 29 \\
\hline hnRNP L & HNRNPL & 64 & 12 & 10 \\
\hline hnRNP M & \begin{tabular}{|l} 
HNRNPM \\
\end{tabular} & 78 & 14 & 8 \\
\hline$h n R N P Q$ & HNRNPQ & 70 & 22 & 44 \\
\hline hnRNP R & \begin{tabular}{|l|} 
HNRNPR \\
\end{tabular} & 71 & 29 & 43 \\
\hline hnRNP U & HNRNPU & 91 & 27 & 39 \\
\hline hnRNP UL-1 & HNRNPUL1 & 72 & 2 & 2 \\
\hline hnRNP UL-2 & HNRNPUL2 & 91 & 9 & 5 \\
\hline PCBP1 & PCBP1 & 38 & 16 & 16 \\
\hline РСBP2 & PCBP2 & 32 & 6 & 8 \\
\hline RALY & RALY & 33 & & \\
\hline
\end{tabular}

\begin{tabular}{|c|c|c|c|c|}
\hline \multirow{3}{*}{$\begin{array}{l}\text { EJC/mRNP } \\
\text { elF4A3 } \\
\text { magh }\end{array}$} & \multirow[b]{2}{*}{ EIF4A3 } & \multirow[b]{2}{*}{47} & \multirow[b]{2}{*}{9} & \multirow[b]{2}{*}{0} \\
\hline & & & & \\
\hline & MAGOH (B) & 17 & 3 & 2 \\
\hline Y14 & RBM8A & 20 & & \\
\hline Pinin & PNN & 82 & 6 & 4 \\
\hline Acinus (fSAP152) & ACIN1 & 147 & & \\
\hline SAP18 & SAP18 & 18 & 1 & 3 \\
\hline UAP56 & \begin{tabular}{|l|} 
DDX39B \\
\end{tabular} & 49 & 15 & 6 \\
\hline RNPS1 & RNPS1 & 34 & 4 & 1 \\
\hline
\end{tabular}

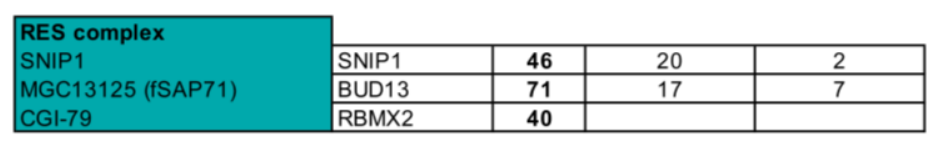

\begin{tabular}{l|l|c|c|c|}
\hline TREX & \multicolumn{5}{|l|}{} \\
\cline { 2 - 5 } THOC1 & THOC1 & $\mathbf{7 6}$ & 14 & 6 \\
\cline { 2 - 5 } THOC2 & THOC2 & $\mathbf{1 8 3}$ & 41 & 11 \\
\cline { 2 - 5 } THOC3 & THOC3 & $\mathbf{3 9}$ & 3 & 2 \\
\cline { 2 - 5 } $\begin{array}{l}\text { Aly/REF (THOC4) } \\
\text { THOC5 (KIAA0983, fSAP79) }\end{array}$ & ALYREF & $\mathbf{2 8}$ & 4 & 5 \\
\cline { 2 - 5 } THOC6 (WDR58, MGC2655) & THOC5 & $\mathbf{7 9}$ & 13 & 8 \\
\cline { 2 - 5 } THOC7 (FLJ23445, fSAP24) & THOC6 & $\mathbf{3 8}$ & 6 & 6 \\
\cline { 2 - 6 } & THOC7 & $\mathbf{2 4}$ & 5 & 2 \\
\hline
\end{tabular}

\begin{tabular}{|c|c|c|c|c|}
\hline \multicolumn{5}{|l|}{ mRNA binding proteins } \\
\hline PABP1 & PABPC1 & 71 & 21 & 21 \\
\hline PABP2 & PABP2 & 33 & & \\
\hline PABPC4 (IPABP, APP1) & PABPC4 & 17 & 16 & 11 \\
\hline YB-1 & YBX1 & 36 & 42 & 35 \\
\hline YBX3 & YBX3 & 40 & 43 & 46 \\
\hline NF45 & ILF2 & 43 & 14 & 21 \\
\hline ELAV & ELAVL1 & 39 & 2 & 6 \\
\hline ASR2B & SRRT & 100 & 40 & 49 \\
\hline KIN17 & KIN17 & 45 & 18 & 0 \\
\hline RBM15 & RBM15 & 106 & 11 & 9 \\
\hline
\end{tabular}




\section{Results}

\begin{tabular}{|c|c|c|c|c|}
\hline & & & & $x-80$ \\
\hline Protein & hGene Name & $\mathrm{kDa}$ & Mock & $\Delta$ Smu1/RED \\
\hline cap binding comple & & & & \\
\hline CBP80 & NCBP1 & 92 & 50 & 51 \\
\hline CBP2O & NCBP2 & 18 & 9 & 10 \\
\hline NCBP3 & NCBP3 & 71 & & \\
\hline
\end{tabular}

\begin{tabular}{|l|l|c|c|c|}
\hline DEXD/H-box RNA Helicases & \multicolumn{5}{|l|}{} \\
\cline { 2 - 5 } p68 (DDX5) & DDX5 & $\mathbf{6 9}$ & 27 & 42 \\
\cline { 2 - 5 } DHX36 & DHX36 & $\mathbf{1 1 5}$ & 31 & 23 \\
\cline { 2 - 5 } DHX38 & DHX38 & $\mathbf{1 4 0}$ & 26 & 14 \\
\cline { 2 - 5 } DDX3X & DDX3X & $\mathbf{7 3}$ & 17 & 19 \\
\cline { 2 - 5 } DDX21 & DDX21 & $\mathbf{8 7}$ & 11 & 0 \\
\cline { 2 - 5 } p72 (DDX17) & DDX17 & $\mathbf{8 1}$ & 17 & 28 \\
\cline { 2 - 5 } DDX9 & DHX9 & $\mathbf{1 4 2}$ & 59 & 49 \\
\hline
\end{tabular}

\begin{tabular}{l|l|l|l|l|}
\hline $\begin{array}{l}\text { Transcription } \\
\text { TCERG1 }\end{array}$ & \multicolumn{5}{|l}{} \\
\cline { 2 - 5 } & TCERG1 & $\mathbf{1 2 4}$ & 12 & 2 \\
\hline
\end{tabular}

\section{Miscellaneous}

YWHAZ

JUP

YWHAE

ENO2

DPYSL2

HSPA1A

HSPA5

YWHAH

ATP1A3

TFIP11

NF110b

LDHB

PAXBP1

PPIA

CALM3

STXBP1

GAPDH

YWHAB

SKIV2L2

ATP1A1

SFN

TPI1

$\mathrm{MDH} 1$

PHF5A

Nucleolin

BCLAF1

ALDOA

SON

PGK1

PRDX1

PIP

NPM1

PRRC2C

ATP5A1

HRNR

FNBP4

NF45

ANXA2

PLP1

IGF2BP3

LYZ

AHNAK

DNA topoisomerase 1

PRCC

ZCCHC8

GOT 1

AZGP1

EXOSC10

ZC $3 \mathrm{H} 11 \mathrm{~A}$

SSB

\begin{tabular}{|c|c|c|c|}
\hline YWHAZ & 28 & 88 & 88 \\
\hline JUP & 66 & 76 & 56 \\
\hline YWHAE & 29 & 66 & 77 \\
\hline ENO2 & 47 & 64 & 56 \\
\hline DPYSL2 & 62 & 61 & 45 \\
\hline HSPA1A & 70 & 51 & 46 \\
\hline HSPA5 & 72 & 51 & 43 \\
\hline YWHAH & 28 & 49 & 54 \\
\hline ATP1A3 & 112 & 47 & 41 \\
\hline TFIP11 & 97 & 41 & 15 \\
\hline $\mathrm{NF} 110 \mathrm{~b}$ & 96 & 40 & 43 \\
\hline LDHB & 37 & 39 & 40 \\
\hline PAXBP1 & 105 & 37 & 24 \\
\hline PPIA & 18 & 35 & 31 \\
\hline CALM3 & 17 & 34 & 21 \\
\hline STXBP1 & 68 & 30 & 18 \\
\hline GAPDH & 36 & 29 & 22 \\
\hline YWHAB & 28 & 28 & 39 \\
\hline SKIV2L2 & 118 & 28 & 35 \\
\hline ATP1A1 & 113 & 27 & 0 \\
\hline SFN & 28 & 25 & 35 \\
\hline TPI1 & 31 & 25 & 30 \\
\hline MDH1 & 36 & 24 & 22 \\
\hline PHF5A & 12 & 24 & 22 \\
\hline $\mathrm{NCL}$ & 77 & 23 & 16 \\
\hline BCLAF1 & 106 & 22 & 15 \\
\hline ALDOA & 39 & 21 & 19 \\
\hline SON & 264 & 20 & 24 \\
\hline PGK1 & 41 & 19 & 14 \\
\hline PRDX1 & 22 & 18 & 21 \\
\hline PIP & 17 & 18 & 15 \\
\hline NPM1 & 33 & 18 & 5 \\
\hline PRRC2C & 317 & 17 & 9 \\
\hline ATP5A1 & 60 & 15 & 9 \\
\hline HRNR & 282 & 15 & 7 \\
\hline FNBP4 & 110 & 15 & 0 \\
\hline NF45 & 43 & 14 & 21 \\
\hline ANXA2 & 39 & 13 & 14 \\
\hline PLP1 & 30 & 13 & 14 \\
\hline IGF2BP3 & 64 & 13 & 12 \\
\hline LYZ & 17 & 12 & 15 \\
\hline AHNAK & 629 & 12 & 3 \\
\hline TOP1 & 91 & 12 & 2 \\
\hline PRCC & 49 & 12 & 2 \\
\hline $\mathrm{ZCCHC8}$ & 79 & 11 & 15 \\
\hline GOT1 & 46 & 11 & 10 \\
\hline AZGP1 & 34 & 11 & 8 \\
\hline EXOSC10 & 101 & 11 & 7 \\
\hline $\mathrm{ZC} 3 \mathrm{H} 11 \mathrm{~A}$ & 89 & 11 & 5 \\
\hline SSB & 47 & 11 & 3 \\
\hline MATR3 & 100 & 10 & 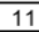 \\
\hline
\end{tabular}


Mass-spectrometric analysis (Table 3.1) further showed that the U2, U5 and U4/U6 di-snRNP proteins, as well as the tri-snRNP specific proteins were equally represented in $\triangle$ Smu1/RED B complexes and kinetically-stalled B complexes. Only few peptides corresponding to Smu1 and RED were detected in $\triangle$ Smu1/RED B complexes, which is consistent with efficient depletion from the extract. Concurrently, levels of the other B-specific proteins remained essentially unchanged, suggesting that the absence of RED and Smu1 did not affect the recruitment of the other B-specific factors. This indicates that the B-specific proteins do not form a pre-assembled complex as is the case for the Prp19/CDC5L complex or the IBC. Instead, they appear to bind the spliceosome as individual proteins or as small subunits like the Smu1/RED dimer.

In contrast, factors known to associate with the spliceosome during and after the activation, such as proteins from

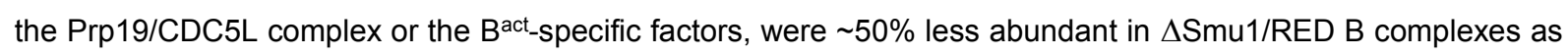
compared with kinetically-stalled B spliceosomes. The presence of these proteins indicates that a small fraction of the kinetically-stalled spliceosomes have already undergone activation. Their clear reduction in the $\triangle$ Smu1/RED B complexes is consistent with stalling of the spliceosome assembly process before the B-to-Bact transition. Taken together, these results show that B complexes assembled in the absence of Smu1 and RED have an snRNA and protein composition highly similar to that of B complexes assembled in mock-depleted extract, suggesting that Smu1 and RED do not exert their function in spliceosome activation indirectly by altering the recruitment of the other B-specific proteins required for activation.

\subsection{The distance between the 5'SS and the branch site, rather than total intron length per se, is the decisive factor for Smu1/RED dependence}

The results accrued so far suggested uniformly that Smu1 and RED are required spliceosome activation essentially when introns reach a critical length of $\sim 80 \mathrm{nt}$. As an intron can be divided into two main segments - the sequence separating the 5'SS and BS and the region between the BS and 3'SS - the question arises whether the intron length per se or alternatively the length of one of these sections is the decisive factor for the strong dependence on Smu1 and RED. To address this question, I truncated the introns of the PM5 150 and PM5-10 150 pre-mRNAs. PM5 150 is a pre-mRNA that contains a 5' exon and a 236 nt long intron, with an extended PY tract of 60 nt (Fig. 3.9A). PM5$10_{150}$ is a derivative of PM5 150 , the PY tract of which has been truncated to $10 \mathrm{nt}$ (Fig. 3.9B). Consequently, the PY tract and intron of PM5-10150 are shorter than those in PM5150 (where the intron length is $166 \mathrm{nt}$ ), while the distance between 5'SS and BS is $150 \mathrm{nt}$ long in both. Comparison of the two pre-mRNAs allows one to determine effects that are based on the distance between BS and 5'SS.

To be further able to assess the importance of the 5'SS-BS distance, the segment of this intron was additionally truncated from 150 to $56 \mathrm{nt}$ in PM5-1056, resulting in an overall intron length of $73 \mathrm{nt}$. The short PY tract of PM5-10 prevents the first splicing step from taking place and results in $\mathrm{B}^{\text {act }}$ complex stalling. As no splicing products or intermediates can be formed with the PM5-10 constructs, only spliceosome assembly was investigated. 


\section{Results}

Native gels showed that Smu1/RED depletion resulted in an increased B complex signal and somewhat diminished $\mathrm{B}^{\text {act }}$ complex signal with PM5-10 150 , indicating that the activation was impaired (Fig. 3.9C). Nevertheless, a strong signal corresponding to the $\mathrm{B}^{\text {act }}$ complex was still obtained, suggesting that the activation was less efficient but not abolished.

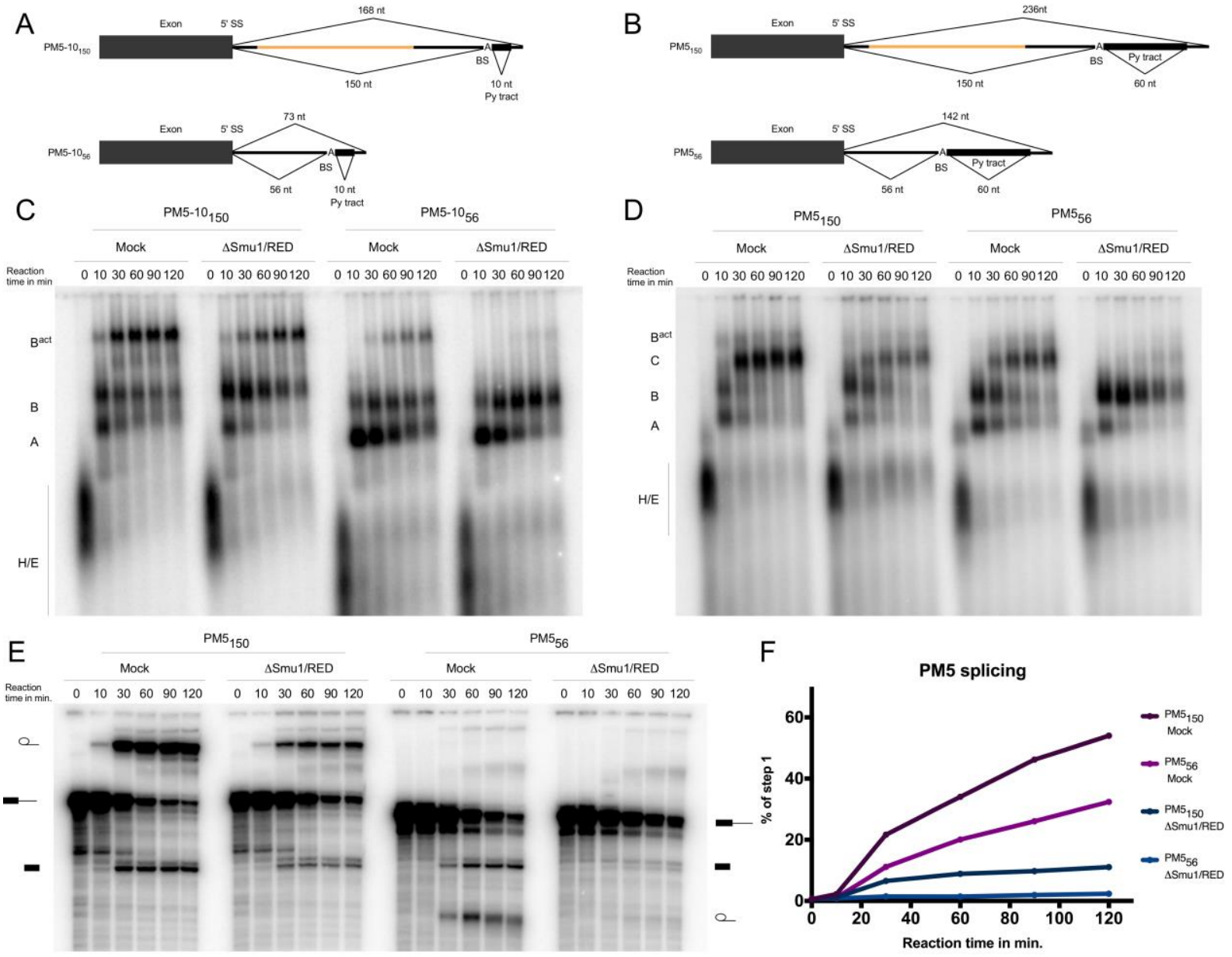

Figure 3.9. PY tract extension does not compensate for a short 5'SS-BS distance. (A) Schematic of PM5-10 constructs. The 5'SSBS distance from PM5-10 150 was shortened to $56 \mathrm{nt}$ in PM5-1056. Reactive sites (5'SS, BP, 3'SS) and the PY tract remained unchanged. In PM5-10 constructs, truncation of the PY tract to $10 \mathrm{nt}$ blocks spliceosome assembly at the $\mathrm{B}^{\text {act }}$ complex level. (B) Schematic of PM5 constructs. The 5'SS-BS distance from PM5 ${ }_{150}$ was shortened to $56 \mathrm{nt}$ in PM556. Reactive sites (5'SS, BP, 3'SS) and PY tract remained unchanged. Owing to a lack of a 3' exon, stalls spliceosome assembly on PM5 constructs stalls at the C complex stage. (C) and (D) Kinetics of spliceosome assembly on PM5-10 or PM5 constructs in the presence or absence of Smu1/RED. ${ }^{32}$ P-labelled pre-mRNAs were incubated under splicing conditions in the presence of Smu1/RED-depleted or mock-depleted HeLa nuclear extract for the times indicated. Spliceosomal complex formation was analysed on a native agarose gel and visualized by autoradiography. The positions of the H/E, A, B, $\mathrm{C}$, and $\mathrm{B}^{\text {act }}$ complexes are indicated on the left. (E) Kinetics of in vitro splicing. The ${ }^{32} \mathrm{P}$-labelled PM5 pre-mRNA was incubated with Smu1/RED-depleted or mock-depleted extract under splicing conditions for the times indicated, separated by denaturing PAGE and visualized by autoradiography. The pre-mRNA and splicing intermediates are indicated on the left and right. (F) Quantification of the kinetics of the formation of splicing intermediates over the reaction time. 
Reduction of the distance between 5'SS and BS in PM5-10 from 150 to $56 \mathrm{nt}$ appeared generally to slow down the transition from one complex to the next under conditions of mock-depletion. In $\triangle$ Smu1/RED extract, however, only traces of activated spliceosomes were detected, while the B complex signal was stably increased even after 120 min, indicating that the B-to-Bact transition was completely blocked.

Truncation of the 5'SS-BS distance in PM5 from 150 to $56 \mathrm{nt}$ resulted in an overall intron length of $142 \mathrm{nt}$, which is still above the determined threshold of $100 \mathrm{nt}$. While the extended PY tract of PM5 constructs allows the formation of activated spliceosomes, the assembly is blocked at the $C$ complex level, owing to the lack of a $3^{\prime}$ exon. As the first splicing step can nonetheless occur, splicing was additionally investigated by denaturing PAGE. Spliceosome assembly on PM5150 appeared less responsive to the Smu1/RED depletion, as a strong increase in B complex signal was only observed after 10 minutes, and it subsequently quickly decreased (Fig. 3.9D). However, the C complex signal in $\triangle$ Smu1/RED extract was significantly decreased compared with mock-depleted extract, suggesting that the assembly was still restricted by the Smu1/RED-depletion. Consistent with this, the first splicing step of PM5 was significantly less efficient without Smu1/RED (Fig. 3.9E); while 55\% of PM5 150 had undergone the first splicing step after 120 minutes in mock-depleted extract, this was only the case for $11 \%$ of PM5-10 150 in extract depleted of Smu1/RED. (Fig. 3.9F). Interestingly, shortening of the PM5 intron between 5'SS and BS decreased the efficiency of spliceosome assembly in mock-depleted extract, with $\sim 45 \%$ less of the splicing intermediates formed after 120 minutes ( 31\%). Remarkably, in the absence of Smu1/RED, spliceosome assembly on PM556 was clearly stalled at the $\mathrm{B}$ complex level and only traces of $\mathrm{C}$ complexes were detected. At the same time, only a very small fraction of the pre-mRNA had been converted to splicing intermediates.

As the PM5 and PM5-10 constructs were similarly affected by the depletion of Smu1 and RED, the distance between the BS and the 3'SS does not appear to be a relevant factor. In contrast, shortening of the 5'SS-BS distance blocked the activation of spliceosomes formed on both PM556 and PM5-1056 indicating that this distance is the decisive factor for the Smu1/RED-dependence of short-intron splicing. Consequently, it is not the intron length per se that determines whether Smu1 and RED are essential, but the distance between the 5'SS and the BS.

\subsection{Cleaving the MINX-80 substrate into two parts restores activation on short introns independently of Smu1/RED}

My results indicate that Smu1 and RED jointly support the activation of the spliceosome, and that this support becomes essential when the distance between 5 'SS and BS reaches a critical length of $\sim 56$ nt or shorter. A reasonable explanation for this length-dependence is that short introns might exercise a physical constraint upon the spliceosome, thereby preventing movements required for the activation. Consequently, releasing the physical constraint exerted through the pre-mRNA on the stalled $\triangle$ Smu1/RED spliceosome (by splitting the short MINX-80 intron between the 5 'SS and BS) should restore the flexibility that the spliceosome needs in order to adopt the conformation required for its activation and for splicing. 


\section{Results}

To test this, I physically separated the intron of the MINX-80 pre-mRNA by transcribing it in two independent parts (MINX-80-cleaved; Fig. 3.10A). The first part comprised Exon1 and 25 nt of the intron downstream of the 5'SS and the second part included the 31 remaining nucleotides upstream of the BS, the BS itself, the PY tract and Exon2.

A

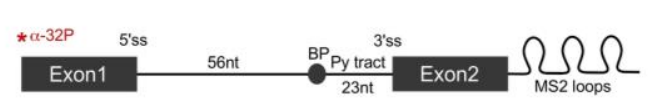

Minx-80

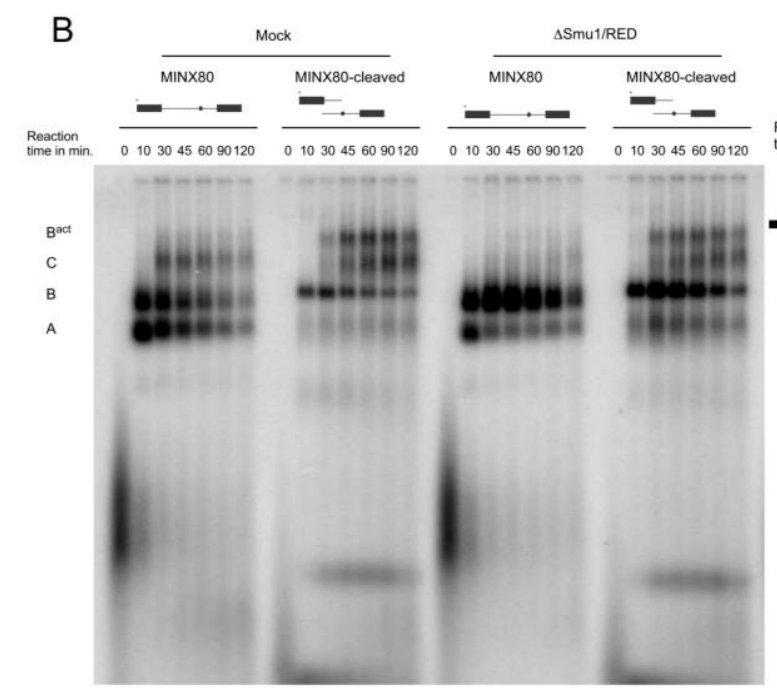

D

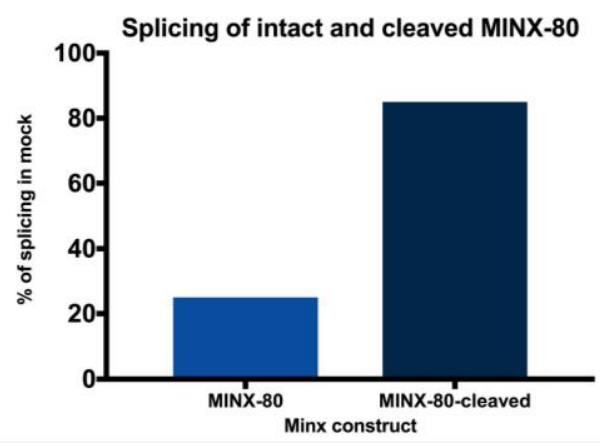

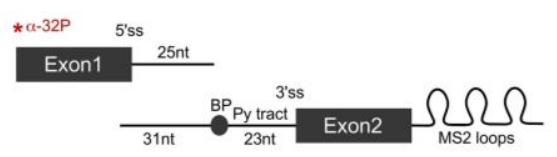

Minx-80-cleaved

C
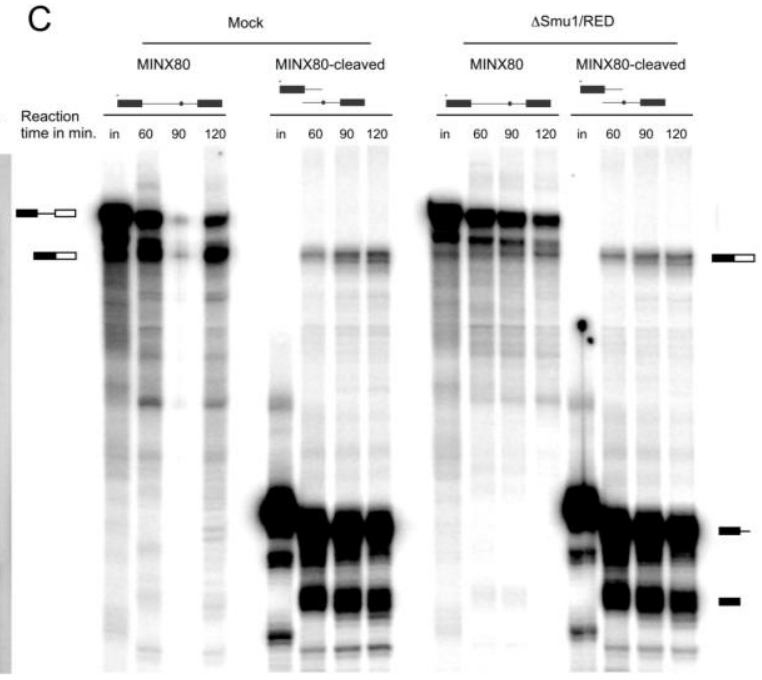

Figure 3.10. MINX-80-cleaved allows spliceosome activation and splicing in the absence of Smu1 and RED. (A) Schematic of MINX80 and MINX-80-cleaved. To obtain the MINX-80-cleaved construct, MINX-80 pre-mRNA was transcribed in two parts. The first contained Exon 1, the 5'SS and $25 \mathrm{nt}$ of the intron. The second part comprised the rest of the intron, including the BP, the PY tract and the 3'SS, as well as Exon 2. The first part was radiolabelled for detection by autoradiography. The two constructs were identical regarding sequence and intron length. Kinetics of spliceosome assembly (B) and in vitro splicing (C) of the intact and cleaved MINX-80 constructs in the presence or absence of Smu1/RED. Pre-mRNAs were incubated under splicing conditions in depleted or mock-depleted extract for the times indicated. (B) Spliceosomal complex formation was analysed on a native agarose gel and visualized by autoradiography. The positions of the $\mathrm{H} / \mathrm{E}, \mathrm{A}, \mathrm{B}, \mathrm{C}$, and $\mathrm{B}^{\text {act }}$ complexes are indicated. (C) RNA was analysed by denaturing PAGE and visualized by autoradiography. The pre-mRNA and splicing intermediates and products are indicated on the right or left. (D) Quantification of pre-mRNA to mRNA conversion in $\triangle$ Smu1/RED extract after 120 min relative to the mock sample. 
Thus, MINX-80 and MINX-80-cleaved have identical sequences but MINX-80-cleaved is split between its 5'SS and BS. The MINX-80 and MINX-80-cleaved pre-mRNAs were tested in in vitro splicing reactions performed with Smu1/RED-depleted or mock-depleted extract, and the splicing outcome and spliceosome assembly were investigated by denaturing and native gel analysis, respectively

As shown in Fig. 3.10B, C and D, B, Bact and C complexes were assembled on both MINX-80 and MINX-80-cleaved in mock-depleted extract, and the spliced products could readily be detected, indicating that both constructs are splicing-competent. However, there appeared to be an accumulation of $\mathrm{B}^{\text {act}} / \mathrm{C}$ complexes with the cleaved versus uncleaved MINX-80 pre-mRNA. Also, less A complexes were detected, likely because they do not withstand the native gel conditions when U1 and U2 bind to physically separated pieces of the pre-mRNA. As described above, spliceosome assembly on MINX-80 pre-mRNA in Smu1/RED-depleted extract was stalled at the B complex level, as indicated by a strong accumulation of $B$ complexes at all times, while the $\mathrm{B}^{\text {act }}$ and $\mathrm{C}$ complex signals were reduced as compared with the mock-depleted sample (Fig. 3.10B). Consistent with this, splicing was $\sim 80 \%$ less efficient in the absence of Smu1 and RED. However, spliceosomes assembled on MINX-80-cleaved could be activated, as strong signals corresponding to $\mathrm{B}^{\text {act }}$ and $\mathrm{C}$ complexes were detected. Also, the spliced product could readily be detected, although its formation was $20 \%$ less efficient than that observed under mock conditions (Fig. 3.10D).

Thus, the release of the physical constraint exerted by the pre-mRNA on the spliceosome in MINX-80-cleaved reactions restored the $B-$-to- $B^{\text {act }}$ transition on short introns in the absence of Smu1/RED, but also the formation of splicing product. This strongly supports the idea that the Smu1/RED complex is essential to promote the adoption of a conformation required for spliceosome activation when short introns exert a physical constraint.

\subsection{Stalled $\Delta$ Smu1/RED B complexes can be activated by addition of the purified dimer}

To obtain a better understanding of why spliceosome assembly is stalled in the absence of Smu1/RED, I tested whether $\triangle$ Smu1/RED B complexes are functional assembly intermediates or dead-end complexes whose splicing activity cannot be restored. Therefore, I assembled stalled B spliceosomes in $\triangle$ Smu1/RED extract for 30 minutes and subsequently added the purified dimer. Concomitantly, a 10-fold excess of unlabelled MINX-80 pre-mRNA was added to sequester newly assembled spliceosomes, and thus ensure that any subsequently formed splicing complexes (i.e., the $\mathrm{C}$ complex) could only be derived from the $\mathrm{B}$ complexes formed before addition of Smu1/RED. Spliceosome assembly was analysed by native gel electrophoresis (Fig. 3.11A).

The signal of the $\triangle$ Smu1/RED-stalled B complexes decreased rather slowly upon addition of High Five Elution Buffer and no $B^{a c t} / C$ complex formation was detected. In contrast 15 minutes after addition of Smu1/RED a signal corresponding to $\mathrm{B}^{\text {act }}$ and $\mathrm{C}$ complexes could be detected while the signal of the stalled $\mathrm{B}$ complexes was significantly reduced after 30 mins. Concomitantly, no clear formation of spliced mRNA or excised intron could be detected at any time after addition of elution buffer to the stalled B complexes (Fig. 3.11B and C). However, when 


\section{Results}

the purified dimer was added, both mRNA and spliced intron were detected. After 120 minutes ca. $40 \%$ of the premRNA had been spliced, which is approximately $50 \%$ of the mock control. This indicates that the stalled $B$ complexes could be chased into active spliceosomes that catalyse splicing. Consequently, the B complexes that accumulate in the absence of Smu1/RED are functional assembly intermediates, which are merely stalled prior to activation.
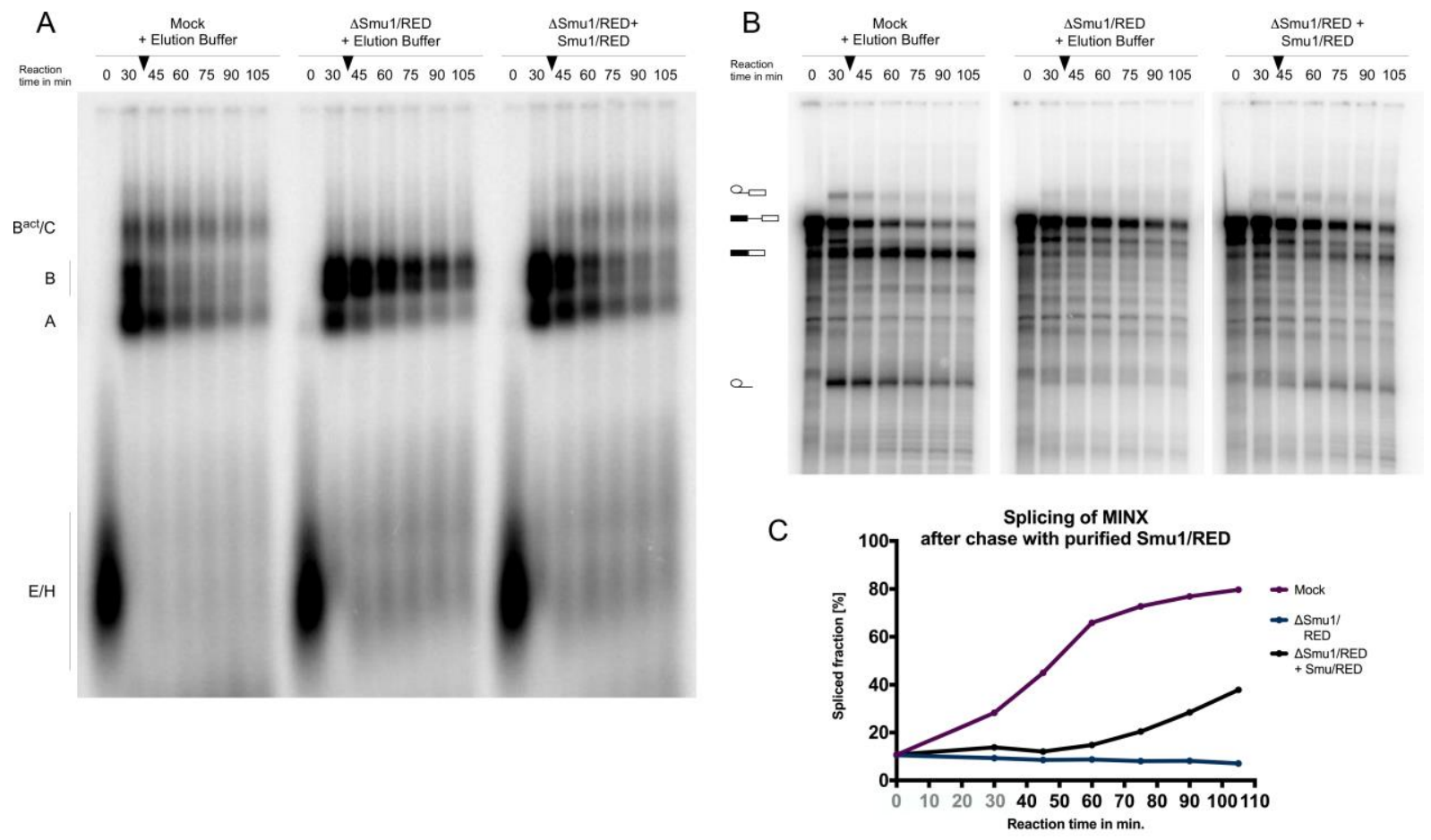

Figure 3.11. $\triangle$ Smu1/RED B complexes can be chased into activated spliceosomes. Kinetics of spliceosome assembly (A) and in vitro splicing (B) after chase with the Smu1/RED dimer, co-expressed in insect cells. $\triangle$ Smu1/RED B complexes were assembled on ${ }^{32} \mathrm{P}$-labelled MINX-80 pre-mRNA under splicing conditions for 30 minutes. Subsequently, purified Smu1/RED dimer or High Five Elution Buffer was added (black arrow) along with a 10-fold excess of cold pre-mRNA. (A) Spliceosomal complex formation was analysed on a native agarose gel and visualized by autoradiography. The positions of the H/E, A, B, C, and Bact complexes are indicated on the left. (B) RNA was analysed by denaturing PAGE and visualized by autoradiography. The pre-mRNA and splicing intermediates and products are indicated on the left. (C) Quantification of the kinetics of the conversion of pre-mRNA to mRNA. Measurements indicated in grey were taken before addition of the elution buffer or purified Smu1/RED dimer, and those indicated in black after this.

\subsection{The interaction between Smu1 and RED is essential for their function in splicing}

Smu1 and RED form a stable module, which was shown to improve the stability of both of these proteins (Spartz et al., 2004). It is likely that the interaction is also important for their function. To investigate how each protein contributes to the dimer's function, I performed a series of complementation experiments using the MINX-80 premRNA and Smu1/RED depleted extract. The tight interaction of Smu1 and RED had made separate investigations in vivo and in vitro impossible. 
Therefore, to examine to which extent each protein contributes to in splicing, I separately expressed and purified recombinant His-Smu1 and His-RED proteins from insect cells. For more detailed functional studies, I additionally generated truncated Smu1 and RED proteins and investigated the effect on spliceosome activation.

\subsubsection{Smu1 and RED can only support the activation co-operatively}

The cryo-EM structure of the human B complex revealed that the interaction of Smu1 and RED is maintained in the spliceosome, suggesting that this interaction is important for their function. Both Smu1 and RED contact U2 and trisnRNP proteins, suggesting that they stabilize the interaction of the $\mathrm{U} 2$ and the tri-snRNP within the B complex, which appears to be important during the spliceosome activation step (Bertram, Agafonov, Dybkov et al., 2017). However, it is unclear whether both proteins are required to provide sufficient stability or if one would be sufficient for this task. To that end, I investigated whether Smu1 or RED alone is able to restore splicing activity, by complementing $\Delta$ Smu1/RED nuclear extract with the individually purified proteins (Fig. 3.12A) and analysing the formation of spliceosome assembly intermediates by native gel electrophoresis (Fig. 3.12B). A strong accumulation of B complexes was detected in Smu1/RED-depleted extract and addition of buffer alone did not relieve this accumulation. Interestingly, when Smu1 or RED were added separately little or no effect on B complex accumulation was observed and spliceosomes did not undergo activation. However, complementation with both single proteins together promoted the B-to-B ${ }^{\text {act }}$ transition as efficiently as the co-expressed dimer did. Concurrently, no splicing product was formed when the single proteins were added, while splicing was very efficient in the presence of both proteins (3.12C) This shows that the individually purified proteins are functional but cannot fulfil their function separately. Furthermore, it shows that Smu1 and RED do not need to be co-expressed to form a functional heterodimer.

While the interaction of Smu1 and RED appears to be crucial for their functionality, it is unclear whether dimer formation is required for their association with the spliceosome. To answer this question, I purified the B complexes assembled in the presence of RED, Smu1 or both proteins and analysed their association with the spliceosome by immunoblotting. Interestingly, addition of each single protein resulted in spliceosomes that migrated more slowly in the glycerol gradient (fractions 14-15) than complexes containing both proteins (fractions 15-16); this resembles the sedimentation behaviour of $\triangle$ Smu1/RED and kinetically-stalled B complexes, respectively (Fig. 3.13A).

Western blot analysis of the purified B complexes showed that a strong signal for both proteins was seen when they were added together (Fig. 3.13B). However, when only one protein was added only low levels of RED were detected and Smu1 was nearly absent. This suggests that the single proteins - especially Smu1 - do not bind efficiently to the spliceosome on their own and, thus, that their interaction is important for efficient association of both proteins. 


\section{Results}

A

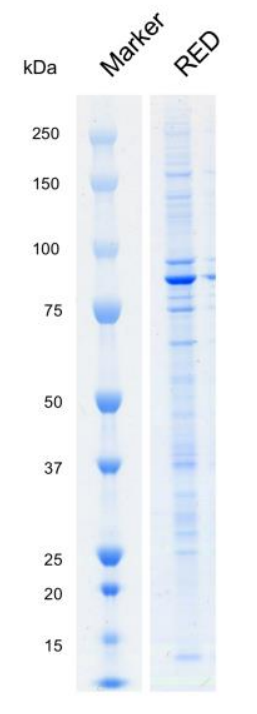

C
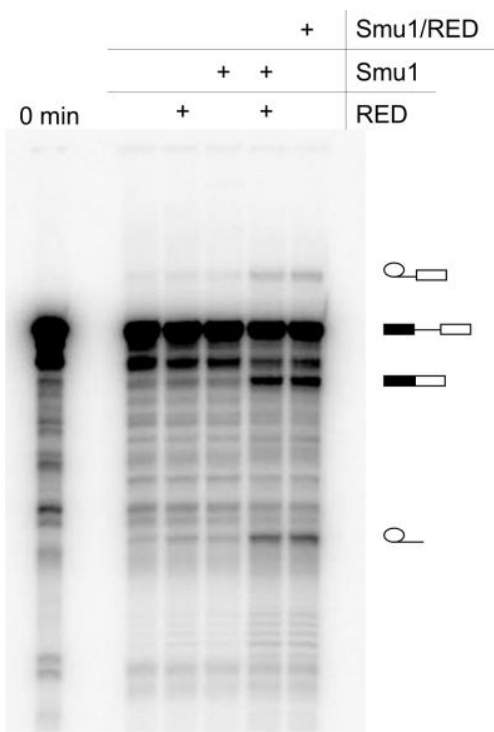

B

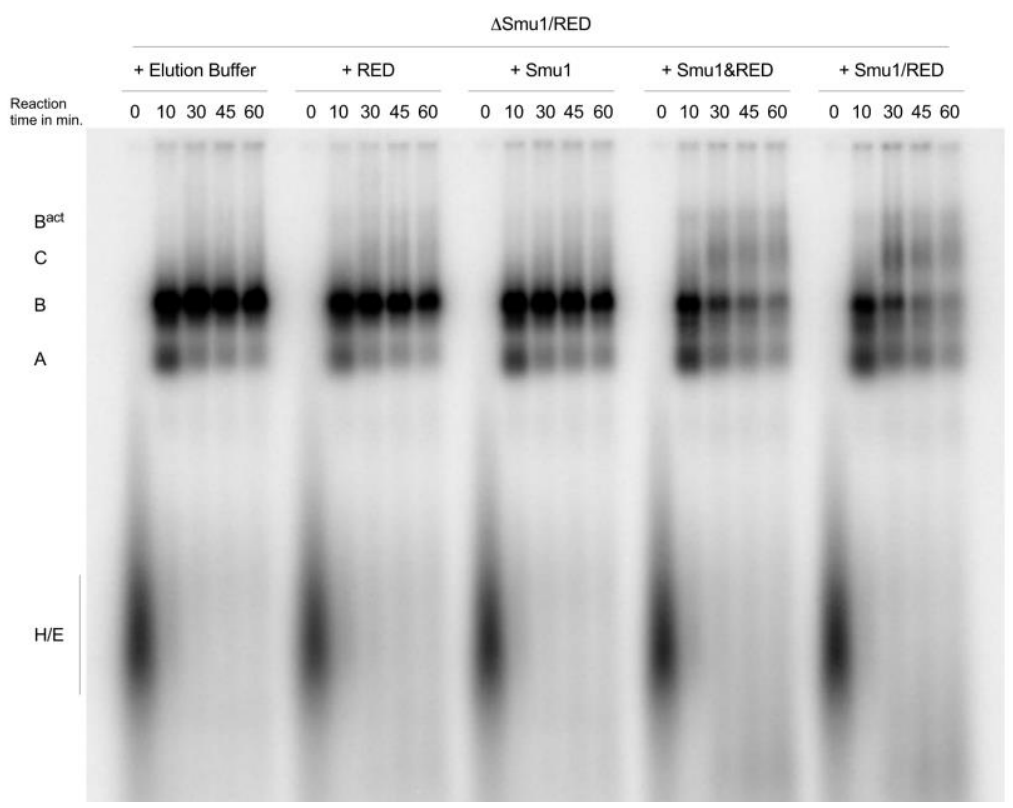

Figure 3.12. The interaction between Smu1 and RED appears to be important for their function. (A) Smu1 and RED, individually expressed and purified from High Five insect cells were separated by SDS-PAGE and stained with Coomassie. (B) Single proteins were added separately or together to Smu1/RED-depleted splicing extract. After 15 minutes incubation under splicing conditions, ${ }^{32} \mathrm{P}$-labelled MINX-80 was added and incubated for the times indicated. Spliceosome assembly was monitored by native gel electrophoresis. As controls, High Five Elution Buffer without protein or the functional co-expressed (Smu1/RED) dimer was added. (C) Splicing of MINX-80 after 60 minutes in the presence of the single proteins or both Smu1 and RED, as indicated in the table above. RNA was analysed by denaturing PAGE and visualized by autoradiography. The pre-mRNA and splicing intermediates and products are indicated on the right. 
A

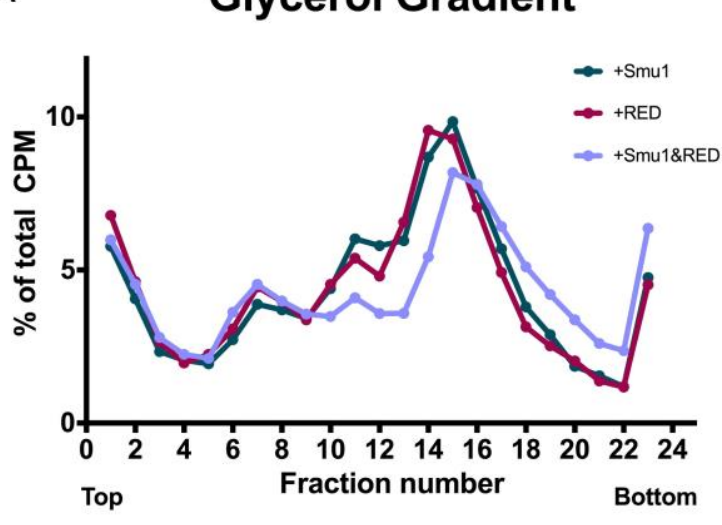

B

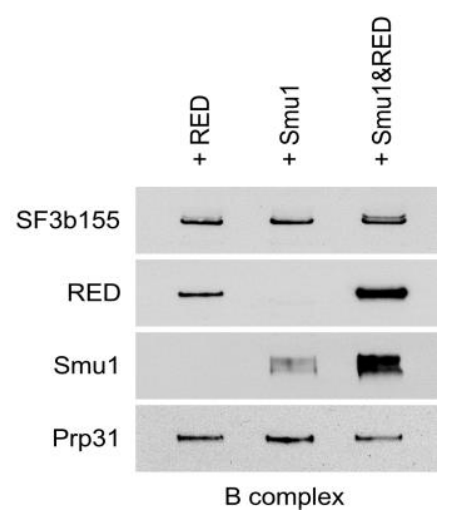

Figure 3.13. The interaction between Smu1 and RED enhances their association with the spliceosome. (A) Purified B complexes assembled in the presence of Smu1, RED or both proteins were seperated by glycerol-gradient ultracentrifugation and their migration was determined by Cherenkov counting. (B) The spliceosomal complexes in the peak fractions (+ Smu1 and + RED, fractions 14 \& 15 ; + Smu1 \& RED, fractions 15 \& 16) were subjected to MS2 affinity purification and analysed by SDS-PAGE followed by immunoblotting using antibodies against SF3b155, RED, Smu1, and Prp31.

\subsubsection{The WD40 domain of Smu1 is required for association of Smu1/RED with the spliceosome}

The structure of the human B complex showed that the WD40 domain of Smu1 is located directly at the interface of the U2 protein SF3B3 and the U5-associated helicase Brr2 (Bertram, Agafonov, Dybkov et al., 2017), which catalyses the unwinding of the U4/U6 duplex during activation. It is possible that the Smu1-WD40 domain stabilizes the positioning of Brr2 relative to its pre-mRNA substrate, thereby regulating the activity of the helicase. Consequently, the Smu1-WD40 domain might be crucial for the function of the Smu1/RED dimer.

To assess the importance of this region for the B-to-Bact transition, I expressed a truncation mutant of Smu1, lacking the C-terminal WD40 domain (Fig. 3.14A). The short remaining fragment still included the RED interaction surface and could be co-purified with full-length (FL) RED (Fig. 3.14B), implying that hetero-dimerization was not inhibited.

As shown in Fig. 3.14C, complementation with Smu1-AWD40/RED did not restore spliceosome activation, as the B complexes remained stable over the entire incubation time, comparable to the negative control (with elution buffer), while barely any later complexes could be detected. Consistently with this, barely any splicing product had been formed in the presence of the Smu1-AWD40/RED dimer (Fig. 3.14D). This suggests that the Smu1-WD40 domain is indeed important for the function of the Smu1/RED dimer. Closer examination of the purified complexes by immunoblotting revealed that the Smu1- WD40/RED dimer could not associate with the spliceosome (Fig. 3.14E). 


\section{Results}

Whereas Smu1/RED was readily detectable in the control complexes, Smu1- $\triangle$ WD40/RED could barely be detected in the purified B complexes. As the association of the dimer appears to be highly dependent on the WD40 domain of Smu1, an investigation of its direct role during the activation of the spliceosome is not possible.

A
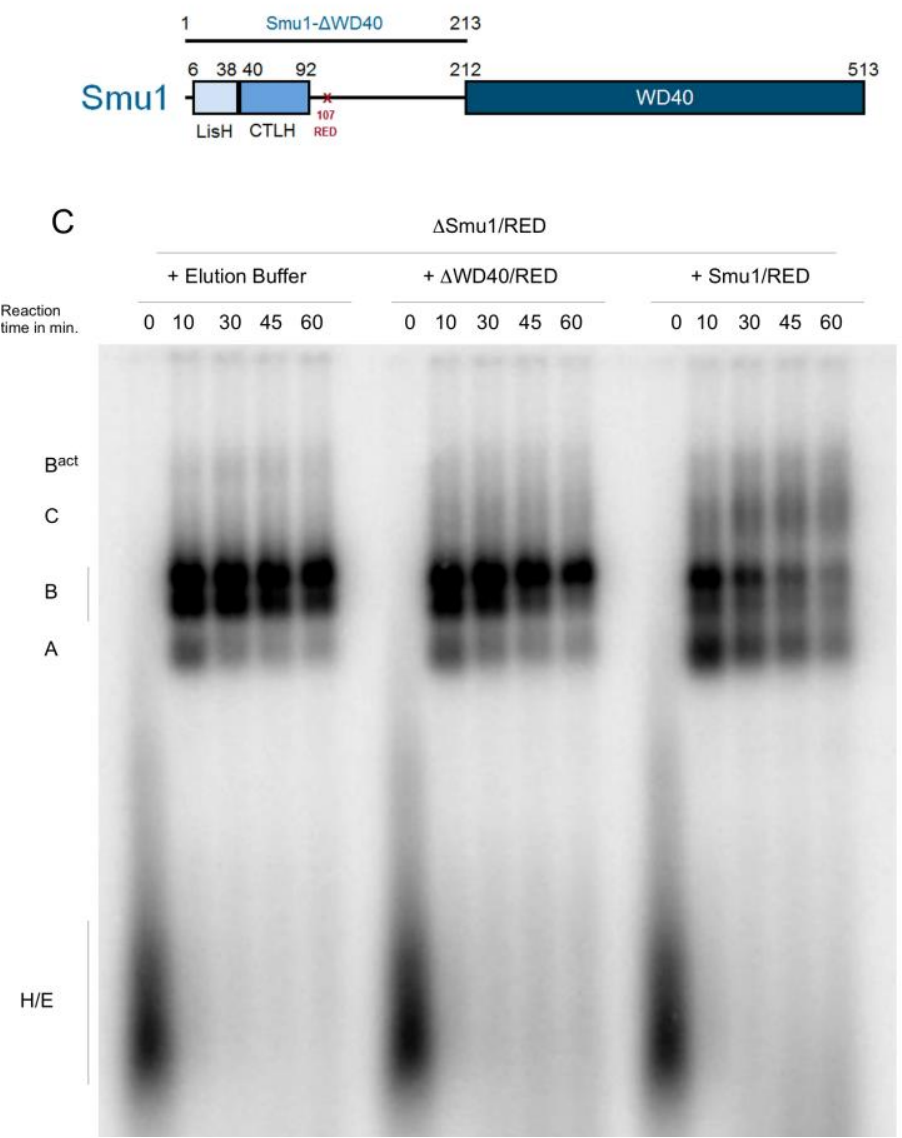

B

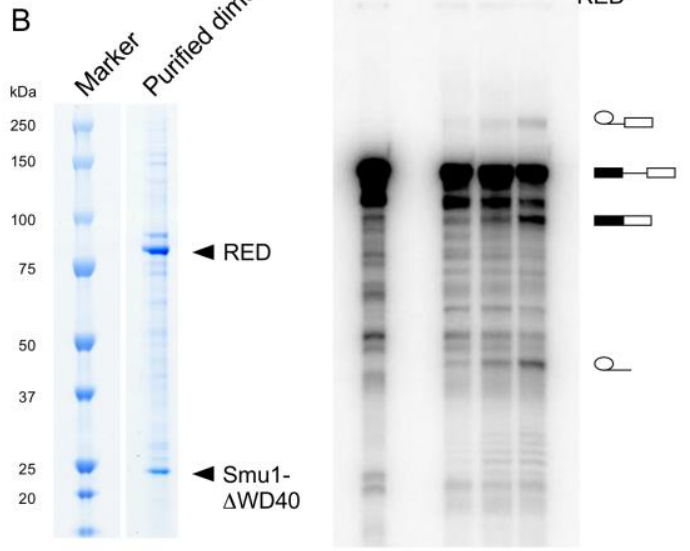

B complex

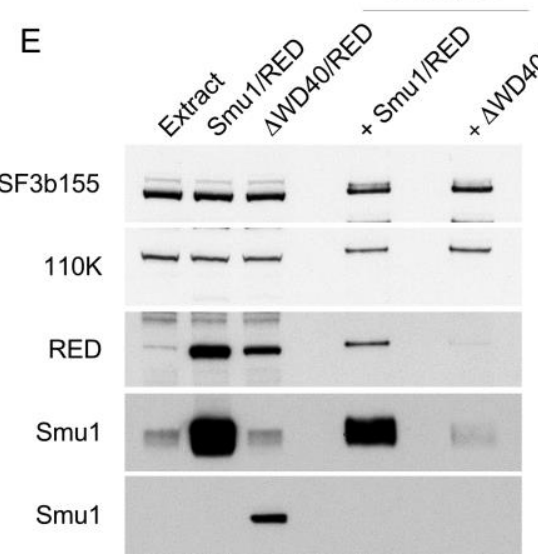

Figure 3.14. The WD40 domain of Smu1 is important for interaction of the dimer with the spliceosome and for its function. (A) Schematic of Smu1. Structural domains are indicated by blue boxes. The amino acid that was crosslinked to RED in the human B complex (Bertram, Agafonov, Dybkov et al., 2017) is marked by a red " $x$ " and indicates the RED interaction region. The part of Smu1 that is included in the Smu1- - WD40 mutant is indicated above. To investigate the function of the WD40 domain of Smu1, a truncated version of the protein (Smu1- WD40) was co-expressed with RED in insect cells. (B) Smu1-LWD40 and RED, co-expressed and purified from High Five insect cells were separated by SDS-PAGE and stained with Coomassie. (C) Kinetics of spliceosome assembly on MINX-80 in the absence/

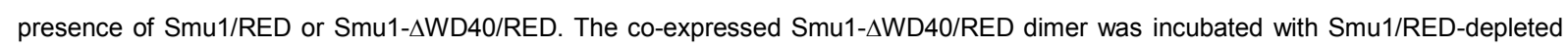
extract under splicing conditions and subsequently with ${ }^{32} \mathrm{P}$-labelled MINX-80 pre-mRNA for the indicated times. The complexes formed were analysed on a native gel. As controls, the buffer without protein or the functional co-expressed dimer was added. (D) Splicing of MINX-80 after 60 minutes in the presence of Smu1/RED or Smu1-AWD40/RED. RNA was analysed by denaturing PAGE and visualized by autoradiography. The pre-mRNA and splicing intermediates and products are indicated on the right. $(E)$ The $B$ complexes assembled in the presence of Smu1/RED or Smu1-AWD40/RED were resolved by glycerol-gradient ultracentrifugation and affinity-purified. Their protein composition was investigated by immunoblotting with the antibodies indicated at the left. Smu1/RED-depleted extract alone or complemented with Smu1/RED or Smu1-AWD40/RED, was loaded a reference for protein migration. 


\subsubsection{Structural requirements of RED for supporting $B^{\text {act }}$ complex formation}
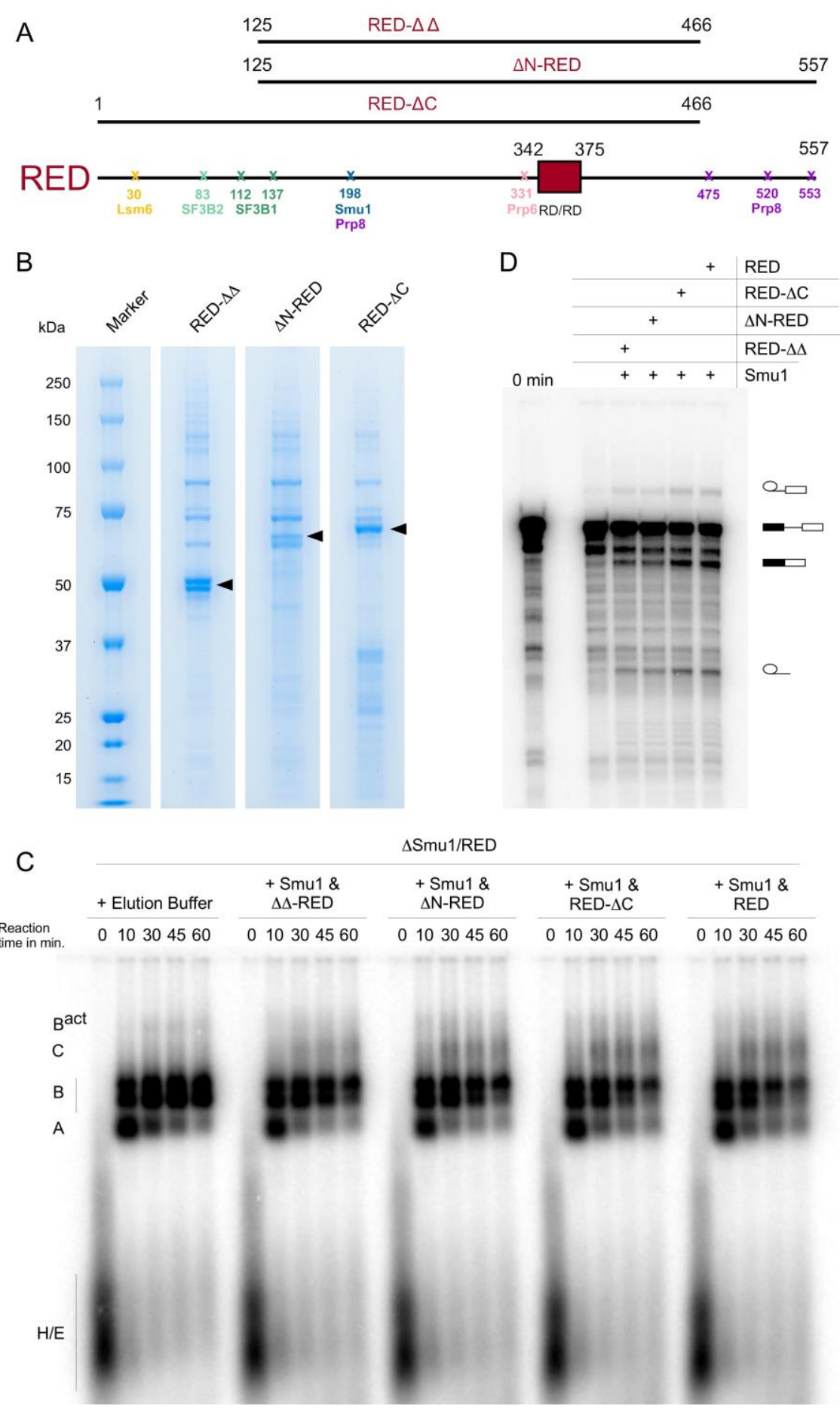

Figure 3.15. RED contributes to the function of the Smu1/RED dimer. (A) Schematic of RED. The amino acids that were crosslinked to other proteins in the human B complex (Bertram, Agafonov, Dybkov et al., 2017) are shown by a coloured " $x$ " indicating the interaction areas. The parts included in the RED- $\Delta \Delta, \Delta \mathrm{N}-\mathrm{RED}$ or RED- $\Delta$ C mutants are shown above. (B) RED- $\Delta \Delta, \Delta \mathrm{N}-\mathrm{RED}$ or RED- $\Delta \mathrm{C}$ mutants, expressed and purified from High Five insect cells were separated by SDS-PAGE and stained with Coomassie. (C) Kinetics of spliceosome assembly on MINX-80 in the absence/presence of Smu1/RED or the indicated Smu1/RED-mutant. Together with Smu1-FL, the mutants were added to Smu1/RED-depleted splicing reactions and the complexes formed were analysed on a native gel. As controls, the buffer without protein or RED-FL was added. (D) Splicing of MINX-80 after 60 minutes in the presence of Smu1 and the different RED mutants as indicated in the table above. RNA was analysed by denaturing PAGE and visualized by autoradiography. The pre-mRNA and splicing intermediates and products are indicated on the right. 


\section{Results}

To determine the role of RED in splicing, the protein was truncated $\mathrm{N}$ - and/or C-terminally (Fig. 3.15A) and added to the splicing reaction together with full-length Smu1. Investigation of the purified RED mutant proteins by SDSPAGE (Fig. 3.15B), revealed bands of the expected sizes for all of them. As could be observed for individually expressed and purified RED, some extra bands could be detected. Interestingly, co-expression with Smu1, did not improve the yield or purity of the protein.

Investigation of spliceosome formation on native gels (Fig. 3.15C) revealed that when the double mutant RED- $\Delta \Delta$ was added, B complexes remained stable for 60 minutes and minor amounts of $\mathrm{C}$ complexes were detectable. When $\triangle \mathrm{N}$-RED or RED- $\Delta \mathrm{C}$ were added, the $\mathrm{B}$ complex signal decreased over time, while a clear $\mathrm{C}$ complex signal appeared after 30 minutes. This indicates that truncation of RED either $\mathrm{N}$ - or $\mathrm{C}$-terminally alone does not abolish the function of the dimer, but that a full-length RED protein leads to more efficient dimer function. Interestingly, investigation of the splicing of MINX-80 revealed that minor amounts of splicing product were formed when RED$\Delta \Delta$ or $\Delta \mathrm{N}-\mathrm{RED}$ was added to the splicing mixture, while splicing in the presence of RED- $\Delta \mathrm{C}$ was similarly efficient as that observed with the full-length protein (Fig.3.15D). This suggests that the N-terminus of RED plays a more important role than its C-terminus.

\subsection{MINX-70 stalls spliceosome assembly after tri-snRNP association but before efficient U1 displacement}

Splicing with the MINX-70 pre-mRNA was completely abolished, consistent with previous studies, indicating that splicing requires a minimal distance between the 5'SS and the BS of 50 nt (Smith \& Nadal-Ginard, 1989; Himmelspach et al., 1991). However, both B and A complexes appeared to form (Fig. 3.5), in contrast to previous results.

A

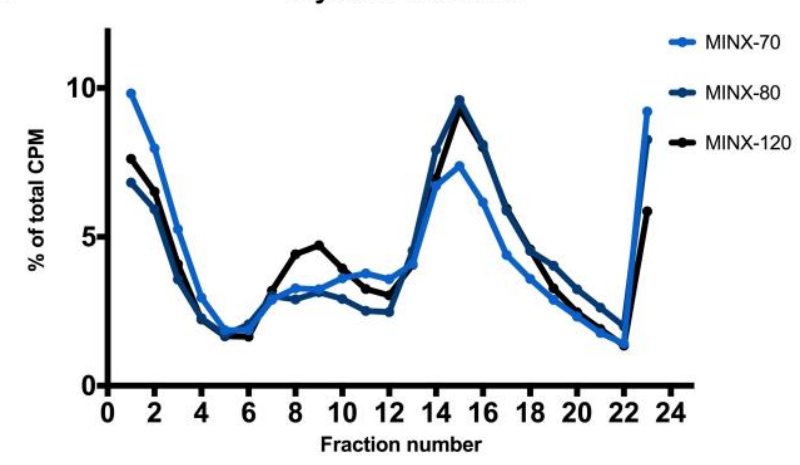

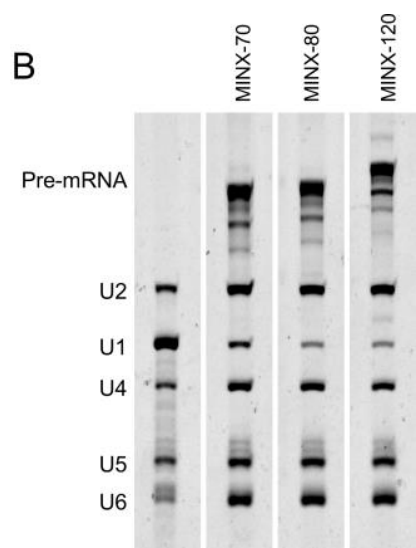

Figure 3.16. Spliceosomes formed on MINX-70 contain higher amounts of U1. (A) Glycerol-gradient sedimentation profile of complexes assembled on MINX-70, MINX-80 and MINX-120. MINX-70 complexes were assembled for 12 min and kinetic $B$ complexes were assembled on MINX-80 or MINX-120 for 8 min under splicing conditions. The migration of the ${ }^{32} \mathrm{P}$-pre-mRNA was determined by Cherenkov counting. (B) snRNA composition of the spliceosomal complexes. The spliceosomal complexes in the peak fractions (fractions 14-16) were subjected to MS2 affinity purification and the RNA extracted from the purified complexes was analysed by denaturing PAGE followed by staining with SYBR Gold. 


\section{Results}

Table 3.2: Protein compositions of spliceosomal complexes assembled on MINX-70, -80 and $\mathbf{- 1 2 0}$. Proteins of the affinity-purified indicated complexes were identified by LC-MS/MS after separation by $4-12 \%$ SDS-PAGE. The number shown is the total number of peptides sequenced for each protein indicated. Proteins are grouped according to function or association. Proteins considered as common contaminants, such as ribosomal proteins and non-reproducibly detected proteins, are not shown.

\begin{tabular}{|c|c|c|c|c|c|}
\hline Protein & hGene Name & $\mathrm{kDa}$ & MINX70 & MINX80 & MINX120 \\
\hline \multicolumn{6}{|l|}{ Sm proteins } \\
\hline$B$ & SNRPB & 24 & 84 & 68 & 72 \\
\hline D1 & SNRPD1 & 13 & 56 & 36 & 44 \\
\hline D2 & SNRPD2 & 14 & 91 & 71 & 88 \\
\hline D3 & SNRPD3 & 14 & 75 & 62 & 78 \\
\hline $\mathrm{E}$ & SNRPE & 11 & 37 & 28 & 22 \\
\hline $\mathrm{F}$ & SNRPF & 10 & 21 & 17 & 11 \\
\hline G & SNRPG & 8 & 21 & 13 & 12 \\
\hline \multicolumn{6}{|l|}{ U1 snRNP } \\
\hline U1-70K & \begin{tabular}{|l|} 
SNRNP70 \\
\end{tabular} & 52 & 30 & 20 & 23 \\
\hline U1-A & SNRPA & 31 & 8 & 4 & 6 \\
\hline U1-C & SNRPC & 17 & & & \\
\hline
\end{tabular}

\begin{tabular}{|c|c|c|c|c|c|}
\hline \multirow{3}{*}{\begin{tabular}{|l}
$17 S^{\prime}$ U2 snRNP \\
U2A' \\
U2B" \\
\end{tabular}} & \multirow[b]{2}{*}{\begin{tabular}{|l|} 
SNRNPA1 \\
\end{tabular}} & \multirow[b]{2}{*}{28} & \multirow[b]{2}{*}{107} & \multirow[b]{2}{*}{93} & \multirow[b]{2}{*}{86} \\
\hline & & & & & \\
\hline & \begin{tabular}{|l|} 
SNRPB2 \\
\end{tabular} & 25 & 29 & 21 & 24 \\
\hline SF3a120 & SF3A1 & 89 & 252 & 192 & 230 \\
\hline SF3a66 & SF3A2 & 51 & 31 & 28 & 34 \\
\hline SF3a60 & SF3A3 & 59 & 153 & 136 & 166 \\
\hline SF3b155 & SF3B1 & 146 & 444 & 438 & 521 \\
\hline SF3b145 & SF3B2 & 100 & 203 & 209 & 237 \\
\hline SF3b130 & SF3B3 & 136 & 529 & 473 & 491 \\
\hline SF3b49 & SF3B4 & 44 & 22 & 33 & 29 \\
\hline SF3b14a/p14 & SF3B14 & 15 & 36 & 36 & 41 \\
\hline SF3b10 & SF3b5 & 10 & 8 & 15 & 9 \\
\hline
\end{tabular}

\begin{tabular}{|c|c|c|c|c|c|}
\hline \multirow{2}{*}{$\begin{array}{l}\text { 17S U2 related } \\
\text { hPRP43 }\end{array}$} & \multirow[b]{2}{*}{ DHX15 } & \multirow[b]{2}{*}{91} & \multirow[b]{2}{*}{103} & \multirow[b]{2}{*}{69} & \multirow[b]{2}{*}{81} \\
\hline & & & & & \\
\hline U2AF65 & U2AF2 & 54 & 45 & 37 & 28 \\
\hline SPF45 & RBM17 & 45 & 20 & 12 & 12 \\
\hline SR140 (fSAPa) & U2SURP & 118 & 39 & 23 & 34 \\
\hline CHERP & \begin{tabular}{|l} 
CHERP \\
\end{tabular} & 104 & 26 & 15 & 28 \\
\hline SF3b125 & DDX42 & 103 & 9 & 3 & 7 \\
\hline U2AF35 & \begin{tabular}{|l|} 
U2AF1 \\
\end{tabular} & 28 & 11 & 14 & 11 \\
\hline SPF31 & \begin{tabular}{|l|} 
DNAJC8 \\
\end{tabular} & 30 & & & \\
\hline hPRP5 & \begin{tabular}{|l|} 
DDX46 \\
\end{tabular} & 117 & 3 & 1 & 5 \\
\hline PUF60 & \begin{tabular}{|l|} 
PUF60 \\
\end{tabular} & 60 & 8 & 4 & 9 \\
\hline SPF30 & SMNDC1 & 27 & 26 & 20 & 6 \\
\hline
\end{tabular}

\begin{tabular}{|c|c|c|c|c|c|}
\hline \multirow{2}{*}{\begin{tabular}{|l|} 
U5 \\
$220 \mathrm{~K}$
\end{tabular}} & \multirow[b]{2}{*}{ PRPF8 } & \multirow[b]{2}{*}{274} & \multirow[b]{2}{*}{876} & \multirow[b]{2}{*}{786} & \multirow{3}{*}{739} \\
\hline & & & & & \\
\hline $200 \mathrm{~K}$ & \begin{tabular}{|l} 
SNRNP200 \\
\end{tabular} & 245 & 976 & 942 & \\
\hline $116 \mathrm{~K}$ & \begin{tabular}{|l} 
EFTUD2 \\
\end{tabular} & 109 & 529 & 475 & 507 \\
\hline $40 \mathrm{~K}$ & \begin{tabular}{|l|} 
WDR57 \\
\end{tabular} & 39 & 117 & 108 & 112 \\
\hline $102 \mathrm{~K}$ & \begin{tabular}{|l|} 
PRPF6 \\
\end{tabular} & 107 & 400 & 349 & 323 \\
\hline $15 \mathrm{~K}$ & \begin{tabular}{|l|} 
TXNL4A \\
\end{tabular} & 17 & 17 & 20 & 20 \\
\hline $100 \mathrm{~K}$ & \begin{tabular}{|l|}
$\mathrm{DDX} 23$ \\
\end{tabular} & 96 & 203 & 108 & 111 \\
\hline $52 \mathrm{~K}$ & CD2BP2 & 38 & 18 & 24 & 21 \\
\hline
\end{tabular}

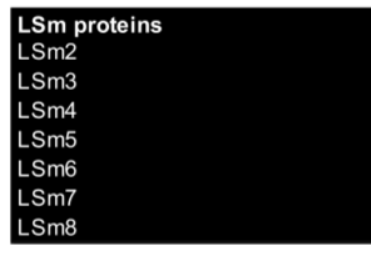

\begin{tabular}{|l|c|c|c|c|}
\hline LSM2 & $\mathbf{1 1}$ & 26 & 28 & 19 \\
\hline LSM3 & $\mathbf{1 2}$ & 8 & 9 & 8 \\
\hline LSM4 & $\mathbf{1 5}$ & 18 & 14 & 10 \\
\hline LSm5 & $\mathbf{1 0}$ & 2 & 1 & 1 \\
\hline LSM6 & $\mathbf{9}$ & 10 & 5 & 7 \\
\hline LSM7 & $\mathbf{1 2}$ & 9 & 10 & 11 \\
\hline LSM8 & $\mathbf{1 0}$ & 26 & 20 & 19 \\
\hline
\end{tabular}

\begin{tabular}{|l|l|c|c|c|c|}
\hline U4/U66 & \multicolumn{7}{|l|}{} \\
& PRPF3 & $\mathbf{7 8}$ & 206 & 201 & 190 \\
\hline $60 \mathrm{~K}$ & PRPF4 & $\mathbf{5 8}$ & 194 & 185 & 189 \\
\hline $20 \mathrm{~K}$ & PPIH & $\mathbf{1 9}$ & 35 & 29 & 24 \\
\hline $15.5 \mathrm{~K}$ & PRPF31 & $\mathbf{5 5}$ & 88 & 88 & 71 \\
\hline & NHP2L1 & $\mathbf{1 4}$ & 13 & 10 & 19 \\
\hline
\end{tabular}

\begin{tabular}{|c|c|c|c|c|c|}
\hline & & & & & \\
\hline & SART1 & 90 & 382 & 274 & 340 \\
\hline \multirow{2}{*}{$\begin{array}{l}110 \mathrm{~K} \\
65 \mathrm{~K} \\
27 \mathrm{~K}\end{array}$} & \begin{tabular}{|l|} 
USP399 \\
\end{tabular} & 65 & 100 & 63 & 58 \\
\hline & & 27 & & & \\
\hline RBM42 & \begin{tabular}{|l} 
RBM42 \\
\end{tabular} & 48 & 7 & 7 & 9 \\
\hline
\end{tabular}




\section{Results}

\begin{tabular}{|c|c|c|c|c|c|}
\hline Protein & hGene Name & $\mathrm{kDa}$ & MINX70 & MINX80 & MINX120 \\
\hline \multicolumn{6}{|l|}{ A complex } \\
\hline THRAP3 & THRAP3 & 109 & 20 & 24 & 18 \\
\hline CCAR1 & CCAR1 & 133 & 9 & 3 & 3 \\
\hline RBM5 & RBM5 & 92 & 22 & 2 & 15 \\
\hline S164 (fSAP94) & RBM25 & 100 & 7 & 5 & 8 \\
\hline RBM10 & RBM10 & 104 & & & \\
\hline SF1 & SF1 & 68 & 4 & 3 & 4 \\
\hline FBP11 & PRPF40A & 109 & 10 & 7 & 5 \\
\hline SF4 & SUGP1 & 72 & 2 & 0 & 1 \\
\hline \multicolumn{6}{|l|}{ Pre-B complex } \\
\hline hPRP4-Kinase & PRPF4B & 115 & 132 & 101 & 89 \\
\hline \multicolumn{6}{|l|}{ B complex } \\
\hline hPRP38 & \begin{tabular}{|l|} 
PRPF38A \\
\end{tabular} & 37 & 79 & 68 & 84 \\
\hline hSnu23 & \begin{tabular}{|l|} 
ZMAT2 \\
\end{tabular} & 24 & 12 & 14 & 15 \\
\hline MFAP1 & MFAP1 & 52 & 43 & 41 & 53 \\
\hline RED & IK & 66 & 120 & 131 & 161 \\
\hline hSmu-1 (fSAP57) & SMU1 & 58 & 132 & 151 & 161 \\
\hline FBP21 & WBP4 & 43 & 15 & 20 & 19 \\
\hline Npw38BP & WBP11 & 70 & 26 & 36 & 57 \\
\hline Npw38 & PQBP1 & 30 & 6 & 3 & 11 \\
\hline UBL5 & UBL5 & 9 & 6 & 6 & 6 \\
\hline
\end{tabular}

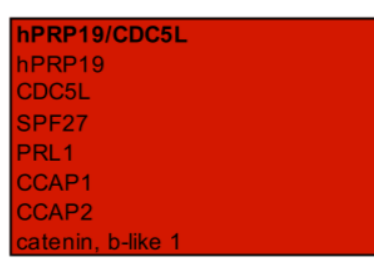

\begin{tabular}{|l|c|c|c|c|}
\hline PRPF19 & $\mathbf{5 5}$ & 107 & 116 & 175 \\
\hline & $\mathbf{9 2}$ & 128 & 122 & 283 \\
\hline BCAS2 & $\mathbf{2 6}$ & 28 & 33 & 52 \\
\hline PLRG1 & $\mathbf{5 7}$ & 37 & 76 & 136 \\
\hline HSPA8 & $\mathbf{7 1}$ & 32 & 37 & 39 \\
\hline CWC15 & $\mathbf{2 7}$ & 7 & 4 & 11 \\
\hline CTNNBL1 & $\mathbf{6 2}$ & 23 & 35 & 58 \\
\hline
\end{tabular}

\begin{tabular}{|l|l|c|c|c|c|}
\hline CRNKL1 (hSYF3) & CRNKL1 & $\mathbf{9 9}$ & & & \\
\cline { 2 - 6 } SKIP & SNW1 & $\mathbf{6 1}$ & 61 & 69 & 154 \\
\cline { 2 - 6 } RBM22 & RBM22 & $\mathbf{4 7}$ & 16 & 23 & 45 \\
\cline { 2 - 6 } $\begin{array}{l}\text { PPlase-like 1 (PPIL1) } \\
\text { G10 (fSAP17) }\end{array}$ & PPIL1 & $\mathbf{1 8}$ & 4 & 4 & 17 \\
\cline { 2 - 6 } & BUD31 & $\mathbf{1 7}$ & 8 & 14 & 23 \\
\hline
\end{tabular}

\begin{tabular}{|l|l|c|c|c|c|}
\hline $\begin{array}{l}\text { IBC } \\
\text { KIAA0560 (fSAP164) } \\
\text { hSyf1 } \\
\text { MGC20398 }\end{array}$ & AQR & $\mathbf{1 7 1}$ & 112 & 150 & 266 \\
\cline { 2 - 7 } \\
hIsy1 (fSAP133) & XAB2 & $\mathbf{1 0 0}$ & 76 & 94 & 209 \\
\hline Zyp-E & ZNF830 & $\mathbf{4 2}$ & 11 & 17 & 22 \\
\hline ISY1 & $\mathbf{3 3}$ & 16 & $\mathbf{2 7}$ & 47 \\
\hline
\end{tabular}

\begin{tabular}{|l|l|c|c|c|c|}
\hline $\begin{array}{l}\text { B }^{\text {act }} \text { complex } \\
\text { KIAA1604 (fSAPb) } \\
\text { hPRP17 }\end{array}$ & CWC22 & $\mathbf{1 0 6}$ & $\mathbf{2 5}$ & 30 & 71 \\
\hline NY-CO-10 & PRPF17/CDC40 & $\mathbf{6 6}$ & $\mathbf{2 7}$ & $\mathbf{2 5}$ & 53 \\
\hline RNF113A & CWC27 & $\mathbf{5 4}$ & 9 & 12 & 22 \\
\hline PPIL2 (Cyp-60) & RNF113A & $\mathbf{3 9}$ & 6 & 5 & 14 \\
\hline PRCC & PPIL2 & $\mathbf{6 0}$ & 29 & 37 & 60 \\
\hline MGC23918 & PRCC & $\mathbf{5 2}$ & 9 & 13 & 23 \\
\hline SRm160 & CCDC12 & $\mathbf{2 1}$ & 12 & 13 & 20 \\
\hline SRm300 & SRRM1 & $\mathbf{1 0 3}$ & 10 & 8 & 25 \\
\hline PPlase-like 3b & SRRM2 & $\mathbf{3 0 0}$ & 45 & 58 & 173 \\
\hline & PPIL3 & $\mathbf{1 9}$ & & & \\
\hline
\end{tabular}

\begin{tabular}{|c|c|c|c|c|c|}
\hline \multirow{3}{*}{$\begin{array}{l}\text { 1st step factors } \\
\text { hPRP2 }\end{array}$} & & & & & \\
\hline & \begin{tabular}{|l|}
$\mathrm{DHX} 16$ \\
\end{tabular} & 119 & 41 & 44 & 115 \\
\hline & GPKOW & 52 & 9 & 15 & 23 \\
\hline CCDC49 & $\begin{array}{l}\text { CWC25 } \\
\end{array}$ & 50 & 7 & 10 & 32 \\
\hline
\end{tabular}

\begin{tabular}{|c|c|c|c|c|c|}
\hline C complex & & & & & \\
\hline GCIP p29 (fSAP29) & SYF2 & 29 & 4 & 6 & 17 \\
\hline C9orf78 (HSPC220) & C9orf7 & 34 & 0 & 0 & 6 \\
\hline WDR70 & WDR70 & 33 & 4 & 2 & 29 \\
\hline NOSIP & NOSIP & 33 & & & \\
\hline PPWD1 (KIAA0073, CyP64) & PPWD1 & 74 & 12 & 6 & 54 \\
\hline PPIL4 & PPIL4 & 35 & 23 & 24 & 44 \\
\hline CDK10 & CDK10 & 35 & & & \\
\hline PPIG (SRcyp) & PPIG & 89 & 4 & 2 & 13 \\
\hline 2nd step factors & & & & & \\
\hline hPRP22 & DHX8 & 139 & 17 & 7 & 63 \\
\hline hPRP18 & \begin{tabular}{|l|} 
PRPF18 \\
\end{tabular} & 40 & & & \\
\hline hSlu7 & SLU7 & 68 & 3 & 0 & 16 \\
\hline hPRP16 & \begin{tabular}{|l|} 
DHX38 \\
\end{tabular} & 140 & 14 & 24 & 43 \\
\hline
\end{tabular}


Results

\begin{tabular}{|c|c|c|c|c|c|}
\hline Protein & hGene Name & $\mathrm{kDa}$ & MINX70 & MINX80 & MINX120 \\
\hline \multicolumn{6}{|l|}{ Putative $C^{*}$ proteins } \\
\hline DDX35 & $\mathrm{DHX35}$ & 79 & 8 & 10 & 23 \\
\hline Abstrakt & DDX41 & 70 & 11 & 6 & 31 \\
\hline C19orf29 (NY-REN-24, cactin) & CACTIN & 89 & 3 & & 25 \\
\hline CXorf56 (FLJ22965) & CXorf56 & 26 & 2 & 0 & 7 \\
\hline FAM50A (XAP5) & FAM50A & 40 & 1 & & 8 \\
\hline
\end{tabular}

\begin{tabular}{|c|c|c|c|c|c|}
\hline SR proteins & & & & & \\
\hline SF2/ASF & SRSF1 & 28 & 71 & 63 & 74 \\
\hline SC35 & SRSF2 & 25 & & & \\
\hline SRp20 & SRSF3 & 19 & 12 & 13 & 15 \\
\hline SRp75 & SRSF4 & 57 & 25 & 24 & 23 \\
\hline SRp40 & SRSF5 & 31 & 36 & 35 & 32 \\
\hline SRp55 & SRSF6 & 40 & 39 & 34 & 36 \\
\hline $9 G 8$ & SRSF7 & 27 & 38 & 33 & 41 \\
\hline SRp46 & SRSF8 & 32 & & & \\
\hline SRp30c & SRSF9 & 26 & 23 & 14 & 20 \\
\hline SRp38 & SRSF10 & 31 & 22 & 16 & 14 \\
\hline SRSF11 & SRSF11 & 54 & & & \\
\hline hTra-2 alpha & TRA2A & 33 & & & \\
\hline hTra-2 beta (SFRS10) & TRA2B & 34 & & & \\
\hline
\end{tabular}

\begin{tabular}{|c|c|c|c|c|c|}
\hline \multirow{2}{*}{$\begin{array}{l}\text { hnRNP } \\
\text { hnRNP A0 }\end{array}$} & \multirow[b]{2}{*}{ HNRNPA0 } & \multirow[b]{2}{*}{31} & \multirow[b]{2}{*}{9} & \multirow[b]{2}{*}{14} & \multirow[b]{2}{*}{22} \\
\hline & & & & & \\
\hline hnRNP A1 & HNRNPA1 & 39 & 74 & 111 & 132 \\
\hline hnRNP A3 & HNRNPA3 & 40 & 14 & 18 & 21 \\
\hline$h n R N P A B$ & HNRNPAB & 35 & 5 & 2 & 2 \\
\hline hnRNP A2/B1 & \begin{tabular}{|l|} 
HNRNPA2B1 \\
\end{tabular} & 37 & 48 & 53 & 62 \\
\hline hnRNP C & HNRNPC & 33 & 39 & 22 & 24 \\
\hline hnRNP D & HNRNPD0 & 24 & & & \\
\hline hnRNP DL & HNRNPDL & 46 & 4 & 2 & 4 \\
\hline$h n R N P F$ & \begin{tabular}{|l} 
HNRNPF \\
\end{tabular} & 46 & 8 & 5 & 7 \\
\hline hnRNP H1 & HNRNPH1 & 49 & 16 & 6 & 10 \\
\hline hnRNP H3 & \begin{tabular}{|l|} 
HNRNPH3 \\
\end{tabular} & 37 & 4 & 1 & 3 \\
\hline$h n R N P K$ & HNRNPK & 51 & 10 & 9 & 10 \\
\hline hnRNP L & HNRNPL & 64 & 7 & 5 & 4 \\
\hline$h n R N P M$ & HNRNPM & 78 & 17 & 15 & 27 \\
\hline$h n R N P Q$ & \begin{tabular}{|l|} 
HNRNPQ \\
\end{tabular} & 70 & 37 & 23 & 28 \\
\hline$h n R N P R$ & HNRNPR & 71 & 45 & 31 & 44 \\
\hline$h n R N P U$ & HNRNPU & 91 & 20 & 12 & 22 \\
\hline hnRNP UL-1 & HNRNPUL1 & 72 & 12 & 7 & 6 \\
\hline hnRNP UL-2 & \begin{tabular}{|l|} 
HNRNPUL2 \\
\end{tabular} & 91 & 6 & 1 & 7 \\
\hline PCBP1 & PCBP1 & 38 & 12 & 14 & 8 \\
\hline РСBP2 & PCBP2 & 32 & & & \\
\hline RALY & RALY & 33 & 5 & 6 & 3 \\
\hline
\end{tabular}

\begin{tabular}{|c|c|c|c|c|c|}
\hline \multicolumn{6}{|l|}{ EJC/mRNP } \\
\hline elF4A3 & EIF4A3 & 47 & 7 & 8 & 16 \\
\hline magoh & MAGOH (B) & 17 & 2 & 0 & 7 \\
\hline Y14 & RBM8A & 20 & 2 & 1 & 4 \\
\hline Pinin & \begin{tabular}{|l|} 
PNN \\
\end{tabular} & 82 & 2 & 1 & 2 \\
\hline Acinus (fSAP152) & ACIN1 & 147 & 22 & 27 & 40 \\
\hline SAP18 & SAP18 & 18 & 2 & 1 & 2 \\
\hline UAP56 & DDX39B & 49 & 6 & 8 & 12 \\
\hline RNPS1 & RNPS1 & 34 & 3 & 4 & 2 \\
\hline
\end{tabular}

\begin{tabular}{|c|c|c|c|c|c|}
\hline \multirow{2}{*}{$\begin{array}{l}\text { RES complex } \\
\text { SNIP1 }\end{array}$} & & & & & \\
\hline & SNIP1 & 46 & 19 & 15 & 23 \\
\hline MGC13125 (fSAP71) & BUD13 & 71 & & & \\
\hline CGI-79 & RBMX2 & 40 & & & \\
\hline
\end{tabular}

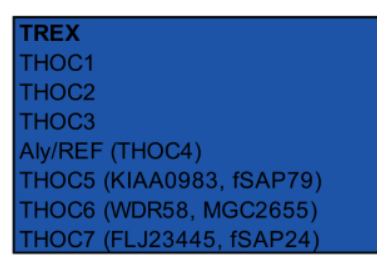

\begin{tabular}{|l|c|c|c|c|}
\hline THOC1 & $\mathbf{7 6}$ & 14 & 13 & 19 \\
\hline THOC2 & $\mathbf{1 8 3}$ & 37 & 21 & 28 \\
\hline THOC3 & $\mathbf{3 9}$ & 5 & 2 & 0 \\
\hline ALYREF & $\mathbf{2 8}$ & 3 & 2 & 5 \\
\hline THOC5 & $\mathbf{7 9}$ & 16 & 12 & 12 \\
\hline THOC6 & $\mathbf{3 8}$ & 6 & 5 & 3 \\
\hline THOC7 & $\mathbf{2 4}$ & 4 & 2 & 2 \\
\hline
\end{tabular}

\begin{tabular}{l}
\hline mRNA binding proteins \\
PABP1 \\
PABP2 \\
PABPC4 (IPABP, APP1) \\
PABPN1 \\
YB-1 \\
YBX3 \\
ELAV \\
ASR2B \\
KIN17 \\
RBM4 \\
RBMX \\
RBM14 \\
RBM15
\end{tabular}

\begin{tabular}{|l|c|c|c|c|}
\hline PABPC1 & $\mathbf{7 1}$ & $\mathbf{2 1}$ & $\mathbf{2 0}$ & 16 \\
\hline PABP2 & $\mathbf{3 3}$ & 6 & 6 & 4 \\
\hline PABPC4 & $\mathbf{1 7}$ & 17 & 10 & 18 \\
\hline PABPN1 & $\mathbf{3 3}$ & 6 & 6 & 4 \\
\hline YBX1 & $\mathbf{3 6}$ & 58 & 60 & 63 \\
\hline YBX3 & $\mathbf{4 0}$ & 53 & 54 & 64 \\
\hline ELAVL1 & $\mathbf{3 9}$ & 13 & 9 & 11 \\
\hline SRRT & $\mathbf{1 0 0}$ & 165 & 89 & 91 \\
\hline KIN17 & $\mathbf{4 5}$ & 15 & 31 & 34 \\
\hline RBM4 & $\mathbf{4 0}$ & 14 & 10 & 12 \\
\hline RBMX & $\mathbf{4 2}$ & 15 & 10 & 11 \\
\hline RBM14 & $\mathbf{6 9}$ & 16 & 11 & 9 \\
\hline RBM15 & $\mathbf{1 0 6}$ & 8 & 4 & 11 \\
\hline
\end{tabular}




\section{Results}

\begin{tabular}{|c|c|c|c|c|c|}
\hline Protein & hGene Name & $\mathrm{kDa}$ & MINX70 & MINX80 & MINX120 \\
\hline \multicolumn{6}{|l|}{ cap bin } \\
\hline CBP80 & NCBP1 & 92 & 49 & 38 & 39 \\
\hline CBP20 & NCBP2 & 18 & 27 & 19 & 21 \\
\hline NCBP3 & NCBP3 & 71 & & & \\
\hline
\end{tabular}

DExD/H-box RNA Helicases p68 (DDX5)

$\mathrm{DHX} 36$

DDX3X

p72 (DDX17)

DDX9

\begin{tabular}{|l|c|c|c|c|}
\hline DDX5 & $\mathbf{6 9}$ & 22 & 17 & 35 \\
\hline DHX36 & $\mathbf{1 1 5}$ & 11 & 9 & 10 \\
\hline DDX3X & $\mathbf{7 3}$ & 13 & 14 & 17 \\
\hline DDX17 & $\mathbf{8 1}$ & 19 & 14 & 19 \\
\hline DHX9 & $\mathbf{1 4 2}$ & 35 & 37 & 59 \\
\hline
\end{tabular}

Transcription

TCERG1

\begin{tabular}{|l|c|c|c|c|}
\hline TCERG1 & $\mathbf{1 2 4}$ & 26 & 46 & 82 \\
\hline
\end{tabular}

Miscellaneous

SPEN

DKFZp762M013

Rnpc2

TFIP11

PAXBP1

ZC3HAV1

SKIV2L2

NF110b

SSB

DKFZp686E2459

SON

PHF5A

Matrin-3

RBBP6

FLJ76180

SETD2

ZCCHC8

LARP7

BCLAF1

Li 3a

PRRC2C

SFSWAP

GAPDH

GPANK1

Peptidyl-prolyl cis-trans isomerase

NF45

EXOSC10

PRRC2A

FUS interacting protein

IGF2BP3

JUP

RSRC1

NPM1

IGF2BP1

STRBP

ZFR

FLJ94609

C17orf85

RNA-binding protein

Heat shock $70 \mathrm{kDa}$ protein $1 \mathrm{~A}$

BCDIN3

SCAF11

HSPA5

$\mathrm{MSI} 2$

PPP1CA

WDR 83

SLC4A1AP

PPP1R8

TTC14

\begin{tabular}{|c|c|c|c|c|}
\hline SPEN & 402 & 71 & 103 & 134 \\
\hline & 87 & 61 & 83 & 173 \\
\hline Rnpc2 & 57 & 60 & 58 & 52 \\
\hline TFIP11 & 97 & 56 & 36 & 51 \\
\hline PAXBP1 & 105 & 53 & 55 & 63 \\
\hline ZC3HAV1 & 101 & 39 & 25 & 29 \\
\hline \multirow[t]{2}{*}{ SKIV2L2 } & 118 & 37 & 36 & 35 \\
\hline & 96 & 29 & 26 & 45 \\
\hline \multirow[t]{2}{*}{ SSB } & 47 & 28 & 29 & 22 \\
\hline & 110 & 27 & 15 & 20 \\
\hline SON & 264 & 24 & 20 & 33 \\
\hline PHF5A & 12 & 24 & 20 & 26 \\
\hline MATR3 & 100 & 23 & 9 & 14 \\
\hline \multirow[t]{2}{*}{ RBBP6 } & 202 & 20 & 22 & 16 \\
\hline & 71 & 18 & 41 & 71 \\
\hline SETD2 & 288 & 18 & 9 & 7 \\
\hline ZCCHC8 & 79 & 17 & 19 & 16 \\
\hline LARP7 & 67 & 17 & 10 & 14 \\
\hline \multirow[t]{2}{*}{ BCLAF1 } & 106 & 16 & 20 & 26 \\
\hline & 35 & 16 & 13 & 15 \\
\hline PRRC2C & 317 & 16 & 11 & 4 \\
\hline \multirow[t]{2}{*}{ SFSWAP } & 105 & 16 & 1 & 3 \\
\hline & 36 & 15 & 17 & 12 \\
\hline \multirow[t]{3}{*}{ GPANK1 } & 39 & 15 & 17 & 12 \\
\hline & 18 & 14 & 16 & 11 \\
\hline & 43 & 14 & 13 & 17 \\
\hline EXOSC10 & 101 & 14 & 6 & 13 \\
\hline \multirow[t]{2}{*}{ PRRC2A } & 229 & 14 & 9 & 4 \\
\hline & 31 & 13 & 8 & 15 \\
\hline IGF2BP3 & 64 & 11 & 7 & 13 \\
\hline JUP & 82 & 11 & 7 & 13 \\
\hline RSRC1 & 39 & 10 & 6 & 11 \\
\hline NPM1 & 33 & 10 & 4 & 4 \\
\hline IGF2BP1 & 63 & 10 & 6 & 9 \\
\hline STRBP & 74 & 9 & 8 & 15 \\
\hline \multirow[t]{6}{*}{ ZFR } & 117 & 9 & 8 & 13 \\
\hline & 66 & 9 & 7 & 12 \\
\hline & 71 & 8 & 6 & 12 \\
\hline & 32 & 7 & 25 & 22 \\
\hline & 77 & 7 & 8 & 10 \\
\hline & 74 & 5 & 12 & 11 \\
\hline SCAF11 & 165 & 4 & 10 & 7 \\
\hline HSPA5 & 72 & 4 & 11 & 11 \\
\hline MSI2 & 35 & 3 & 18 & 9 \\
\hline PPP1CA & 38 & 3 & 13 & 11 \\
\hline WDR83 & 34 & 3 & 2 & 11 \\
\hline SLC4A1AP & 89 & 2 & 10 & 24 \\
\hline \begin{tabular}{|l|} 
PPP1R8 \\
\end{tabular} & 38 & 2 & 5 & 12 \\
\hline TTC14 & 88 & 1 & & 14 \\
\hline
\end{tabular}


Interestingly, B complex formation on MINX-70 featured a migration behaviour similar to that of kinetic B complexes assembled on MINX-80 and MINX-120, with the main peak in fractions 14-16 (Fig. 3.16A). Complexes affinitypurified from the peak fractions 14-16 contained stochiometric amounts of U2, U4, U5 and U6 for all of the premRNAs (Fig. 3.16B), while U1 was enriched in MINX-70 spliceosomes, as compared with complexes assembled on MINX-80 and -120 . This suggests that MINX-70 indeed allows association of the tri-snRNP with the A complex, but the release of $\mathrm{U} 1$ is impaired. Interestingly, the protein composition of MINX-70-associated spliceosomes was barely distinguishable from kinetic B complexes assembled on MINX-80 (Table 3.2). Furthermore, the MINX-70 and MINX-80 complexes differed from B spliceosomes assembled on MINX-120 only in reduced levels of later splicing factors such as the Prp19/CDC5L and intron binding complex as well as B ${ }^{\text {act }}$, first-step, C and second-step factors.

The presence of $\mathrm{U} 4, \mathrm{U} 5$ and $\mathrm{U} 6$ as well as tri-snRNP proteins indicates that the tri-snRNP is able to associate with A complexes assembled on MINX-70. The enrichment of the U1 snRNA compared with kinetic B complexes is reminiscent of the pre-B complex. This recently identified intermediate complex includes a loosely associated trisnRNP before its stable integration by Prp28-driven removal of U1 from the 5'SS, resulting in the formation of a stable 5'SS/U6 ACAGAG helix. However, the B-specific proteins, which have been shown to be absent in pre-B complexes were highly abundant in spliceosomes assembled on MINX-70. It is possible that the short intron found in MINX-70 impairs the formation of a stable 5'SS/U6 ACAGAG helix, which might be formed only in equilibrium and still promote the binding of the B-specific proteins. Thus, MINX-70 might allow the formation of an intermediate spliceosome between the pre-B and B complexes.

\subsection{Smu1 and RED are required for spliceosome activation in vivo}

In vitro experiments pointed to a connection between Smu1/RED-induced defects in splicing and a delayed or stalled B-to $\mathrm{B}^{\text {act }}$ transition, which was characterized by an accumulation of the B complex. To determine whether the changes in splicing observed upon Smu1 and RED depletion were also caused by impaired activation of the spliceosome in vivo, I depleted each protein from HeLa cells by RNAi and investigated the effect on spliceosome assembly. For each protein, two siRNAs with different target sequences were tested, which gave comparable results in all cases. Thus, only one siRNA was used in the subsequent experiments.

As the investigation of endogenous spliceosomes requires tools that allow their detection in their cellular environment, I took advantage of the fact that Prp31 and SF3b155 are specifically phosphorylated in assembled B complexes or activated $B^{\text {act }}$ spliceosomal complexes, respectively (Fig. 3.17). The specificity of the P-SF3b155specific antibody for $\mathrm{B}^{\text {act }}$ complexes has already been verified in vitro and in vivo (Girard et al., 2012).

In addition, in vitro studies showed that Prp31 is specifically phosphorylated in assembled B spliceosomes (Schneider et al., 2010) and dissociates from the spliceosome during activation (Agafonov et al., 2011). 


\title{
Results
}

Therefore, antibodies generated against these two phosphopeptides are excellent markers for assembled B and $\mathrm{B}^{\text {act }}$ spliceosomes in vivo.

As a negative control (CTRL), an siRNA without a target in mammalian cells was transfected into HeLa cells. Furthermore, Prp38 and MFAP1 were depleted for comparison. Prp38 has been shown in yeast and human (Xie et al., 1998; Schütze et al., 2016) to be required for spliceosome activation, while Spp381, the yeast homologue of MFAP1 has been proposed to interact with Prp38 and to contribute to its function in splicing (Lybarger et al., 1999; Ulrich \& Wahl, 2017). It is likely that MFAP1 also supports the function of Prp38 in humans. Thus, removal of these two proteins served as a positive control for defects in spliceosome activation.

After lysis, the transfected cells were fractionated into a soluble nucleoplasmic and an insoluble chromatin fraction by centrifugation. As splicing occurs predominantly co-transcriptionally, the majority of spliceosomes are associated with the chromatin through the nascent transcript and the RNA polymerase II and are thus associated with the chromatin fraction (Pandya-Jones \& Black, 2009; Girard et al., 2012). After solubilisation of the chromatin by sonication, both fractions were separated by SDS-PAGE and transferred to a nitrocellulose membrane for immunoblotting.

Phosphorylation of Prp31

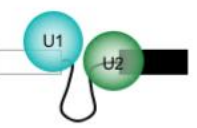

A complex
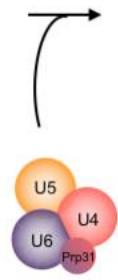

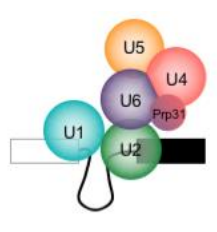

Pre-B complex

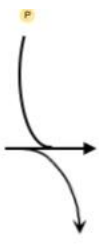

(U1)

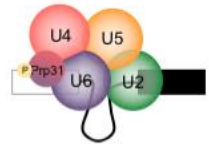

B complex
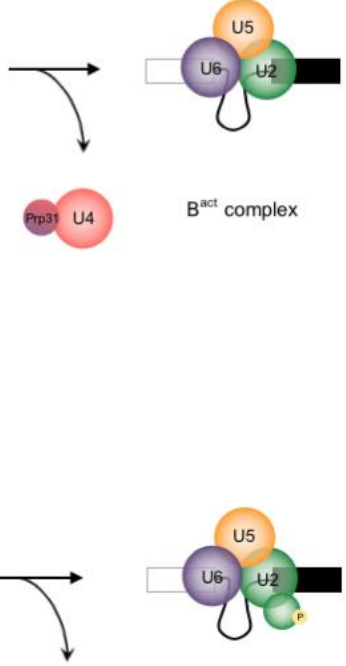

$\mathrm{B}^{\text {ant }}$ complex

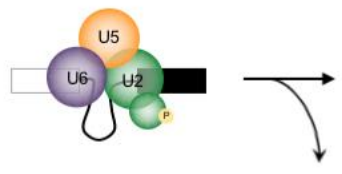

(U4)

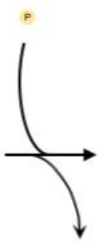

B complex

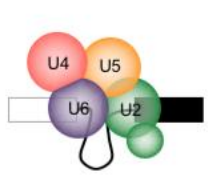

.

\author{
(1)
}


A

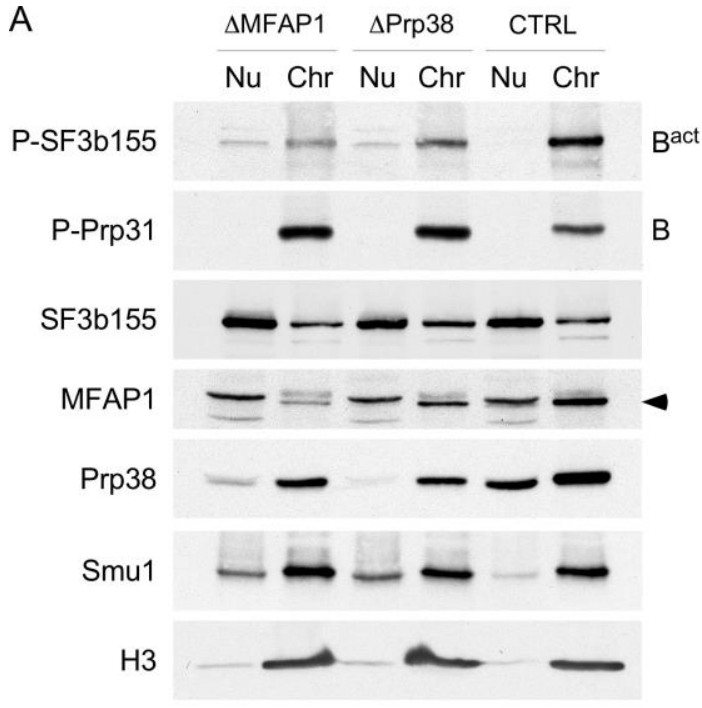

C

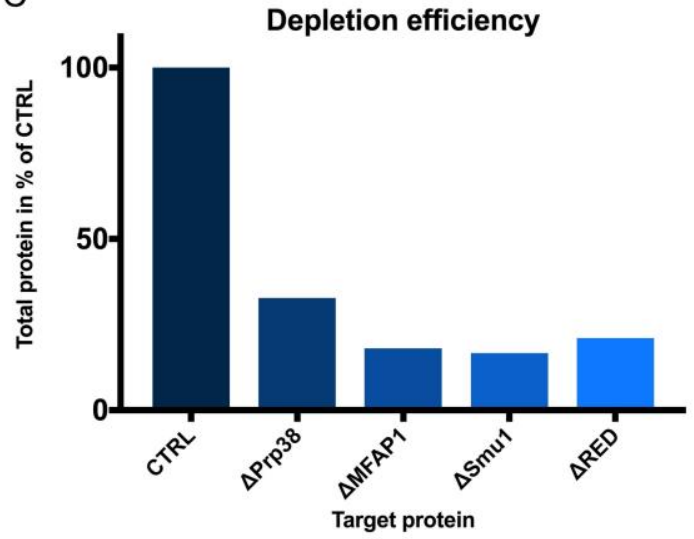

E

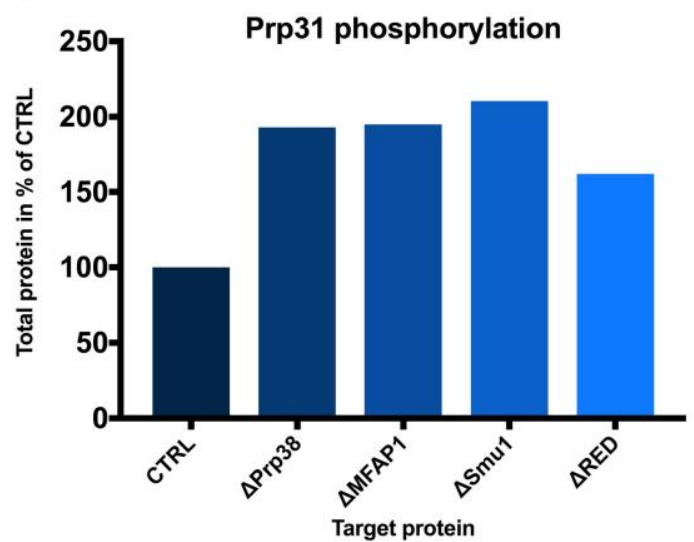

B

$\triangle R E D \quad \triangle S m u 1 \quad$ CTRL

$\mathrm{Nu}$ Chr $\mathrm{Nu} \mathrm{Chr} \mathrm{Nu} \mathrm{Chr}$

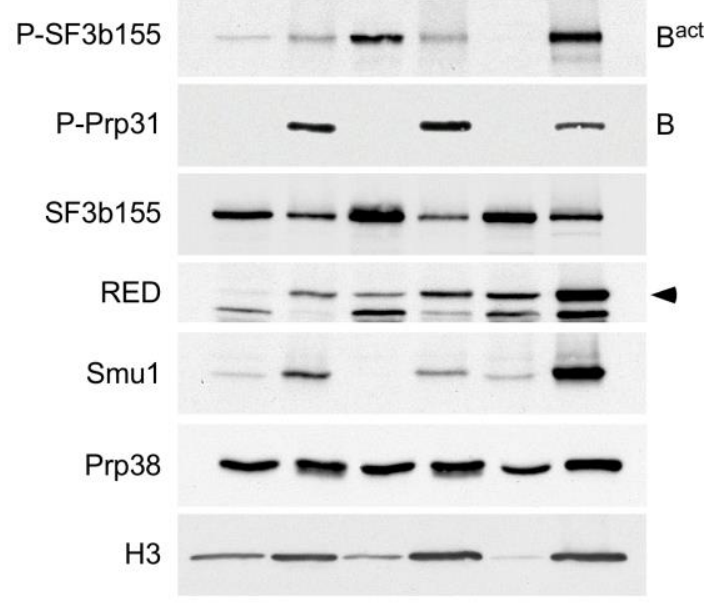

D

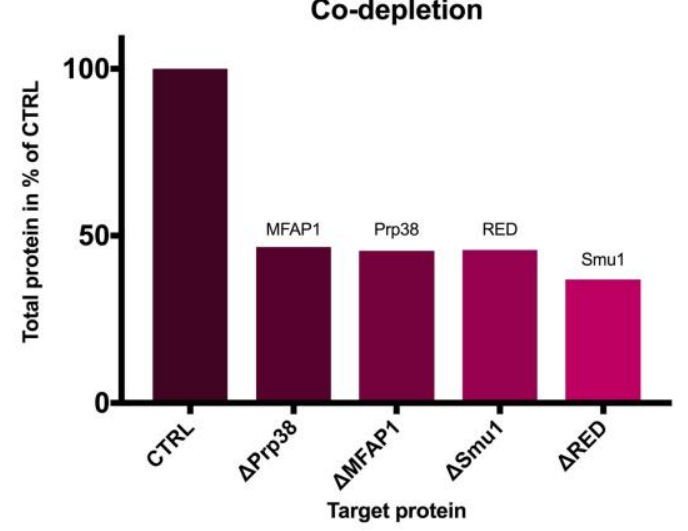

$\mathrm{F}$

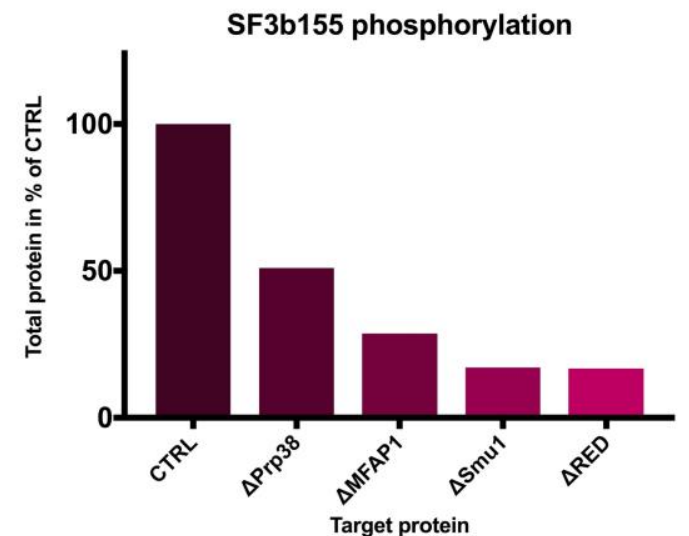

Figure 3.18. siRNA-mediated depletion of Prp38, MFAP1, Smu1 and RED results in altered phosphorylation of Prp31and SF3b155. Western blot analysis of MFAP1- or Prp38-depleted (A) or RED- or Smu1-depleted (B) cells. HeLa cells were lysed and fractionated into a soluble nulceoplasmic $(\mathrm{Nu})$ fraction and an insoluble chromatin $(\mathrm{Chr})$ fraction. Proteins were separated by SDS-PAGE (10-15\% step gel) and blotted onto a nitrocellulose membrane. Immunodetection was performed with antibodies specific for the proteins indicated. (C) Quantification of the total protein level after depletion of the respective protein. The protein level was related to its level in CTRL cells. (D) Depletion of MFAP1/Prp38 or RED/Smu1 resulted in co-depletion of the respective partner. The total protein levels of the distinct proteins after depletion of their respective binding partner were quantified and related to their levels in CTRL cells. (E) Quantification of chromatinassociated phosphorylation signals of SF3b155. (F) Quantification of chromatin-associated phosphorylation signals of Prp31. 


\section{Results}

As shown in Fig. 3.18A, Prp38 and MFAP1 are found in both the nucleoplasmic and chromatin factions under control conditions, with the majority being chromatin associated. siRNA treatment reduced significantly the total protein levels of Prp38 and MFAP1, by 70-80\% (Fig. 3.18C) and appeared to result in recruitment of the remaining protein to the chromatin, consistent with involvement in co-transcriptional splicing. Interestingly, depletion of one of these proteins caused a $\sim 55 \%$ co-depletion of the other (Fig. 3.18D). The two proteins were shown to interact within the B complex and probably also interact before association with the spliceosome (Bertram, Agafonov, Dybkov et al., 2017).

Prp38 and MFAP1 depletion further resulted in a 2-fold increase of P-Prp31 signal concomitant with a 50-75\% decrease of P-SF3b155 signal (Fig. 3.18E and F). This indicates impaired formation of the $\mathrm{B}^{\text {act }}$ complex, resulting in accumulation of its precursor, the B complex. As a function in spliceosome activation has been suggested for the two proteins, this result is consistent with previous findings and provides supportive evidence that the phosphospecific antibodies monitor the correct spliceosomal intermediates in vivo.

Depletion of Smu1 and RED gave similar results (Fig. 3.18B). Upon depletion total protein levels of Smu1 and RED were reduced by $80-85 \%$ (Fig. 3.18C), each concomitant with a 55-65\% co-depletion of the other protein (Fig. 3.18D). This is consistent with the previous observation that Smu1 and RED form a heterodimer. (Spartz et al., 2004; Ulrich et al., 2016; Bertram, Agafonov, Dybkov et al., 2017).

Importantly, Prp31 and SF3b155 phosphorylation patterns were similar to those in $\triangle \mathrm{Prp} 38$ and $\triangle \mathrm{MFAP} 1$ cells. While the chromatin-associated P-SF3b155 signal was decreased by $\sim 80 \%$ in Smu1- and RED- depleted cells, the P-Prp31 signal was increased by 75-100\% (Fig. 3.18E and F). However, Prp38 levels were not affected by knockdown of Smu1 or RED. This suggests that Smu1 and RED, similar to several other B-specific proteins, are involved in spliceosome activation also in vivo.

To make sure that the increased P-Prp31 signal corresponded to assembled B complexes, I immunopurified these spliceosomes from the chromatin fraction of the transfected cells. The used procedure was adapted from Girard et al., 2012, where endogenous $B^{\text {act }}$ complexes were investigated. As shown in Fig. 3.19, this procedure is based on the principles used in chromatin immunoprecipitation (ChIP) and uses the DMS protein-protein crosslinking of the chromatin fraction to keep the spliceosomes intact during the subsequent sonication step. Sonication results in fragmentation of the chromatin, which singularizes the spliceosomal particles. The sheared chromatin fraction was subjected to glycerol-gradient centrifugation and the migration of P-Prp31 (or P-SF3b155) was monitored by western blotting (Data not shown). The RNA was extracted from the peak fractions and analysed by Northern blotting by using a mixture of the radioactively-labelled snRNAs as probes. 
Results

I: Pre-shearing
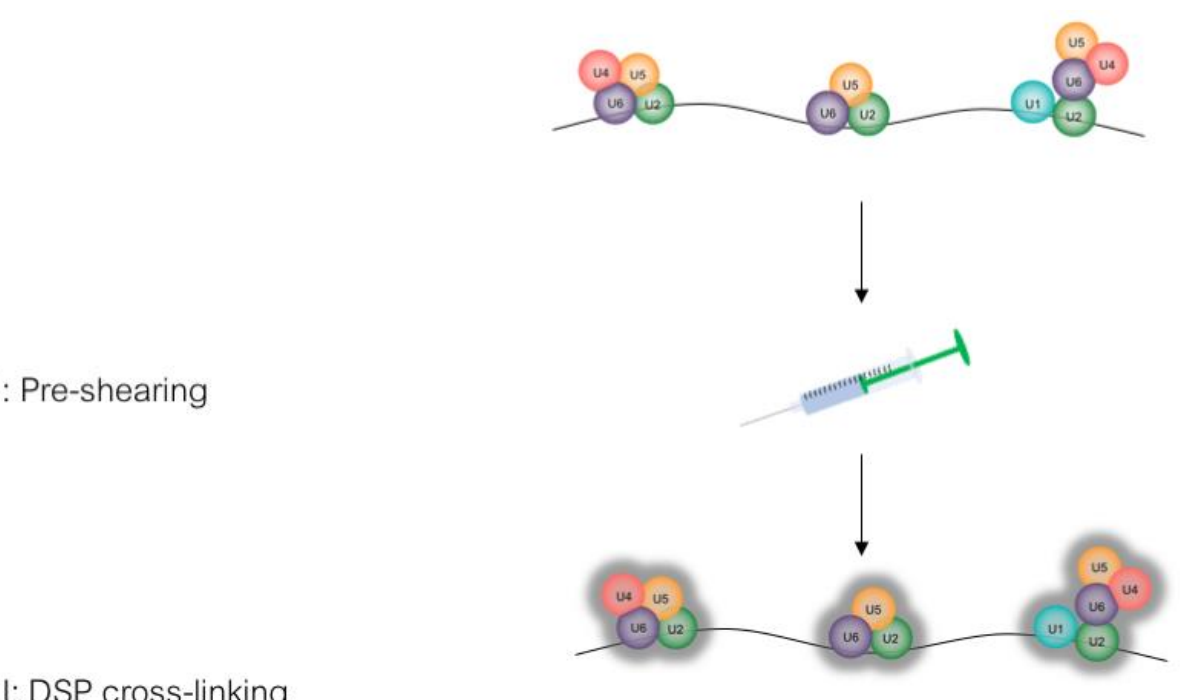

II: DSP cross-linking

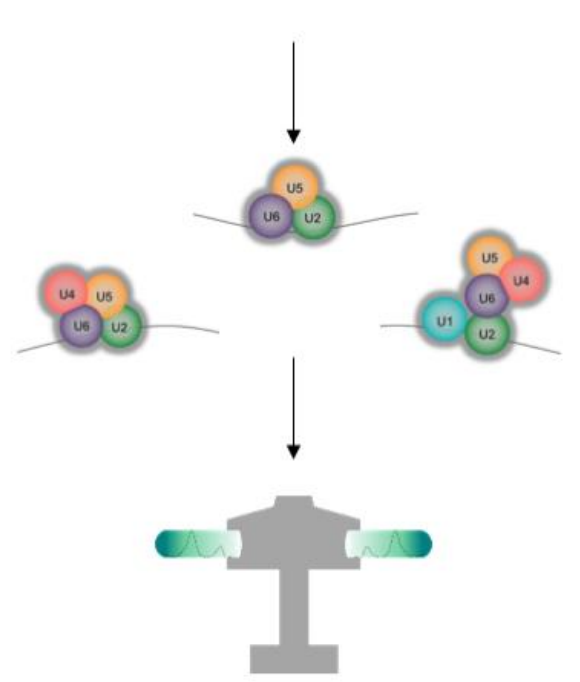

IV: Gradient sedimentation

V: Immunoprecipitation

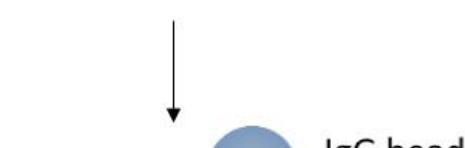

VI: Northern blotting

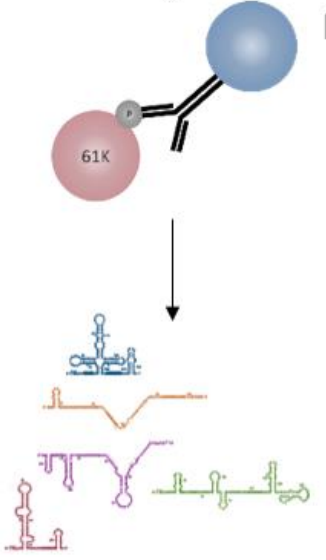

Figure 3.19. Procedure used for the purification of endogenous spliceosomes. The purification procedure included the isolation of the chromatin fraction from HeLa cells followed by rough pre-shearing via passing through a needle in order to disentangle the chromatin. Subsequent DSP crosslinking ensured the maintenance of the spliceosomes integrity during the sonication treatment, which served to separate the spliceosomes through chromatin fragmentation. The spectrum of different complexes was then resolved by glycerol gradient sedimentation. Via immunoprecipitation, the particles of interest were purified from the corresponding fractions and the precipitate's RNA composition was analysed by northern blotting. 


\section{Results}

As shown in Fig. 3.20A, the P-Prp31-specific antibody purified complexes containing all snRNAs from untreated HeLa cells. In contrast, in spliceosomes purified with the P-SF3b155-specific antibody, U1 and U4 were underrepresented, which is consistent with the snRNA composition of activated spliceosomes. Although U1 is not found in in vitro assembled B spliceosomes, it is likely that the P-Prp31-associated complexes are indeed endogenous $\mathrm{B}$ complexes and that the presence of $\mathrm{U} 1$ is an artefact of the purification procedure.

Investigation of the snRNA composition of P-Prp31-associated spliceosomal particles purified from cells depleted of Prp38, MFAP1, Smu1 or RED revealed the presence of all five snRNAs, as it was the case for the control cells (Fig. 3.20B). This supports the idea that the enriched P-Prp31 signal detected in the chromatin fraction of Prp38-, MFAP1-, Smu1- and RED-depleted cells derives from assembled B spliceosomal complexes and is consistent with my in vitro results indicating that Smu1 and RED promote the B-to-Bact-transition during spliceosome assembly.

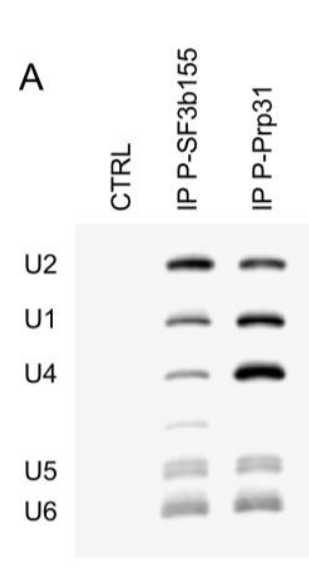

Northern blot

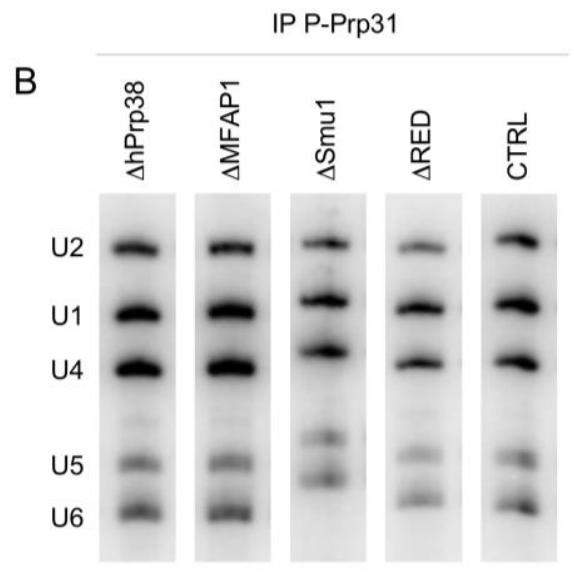

Northern blot

Figure 3.20. RNA composition of endogenous spliceosomes precipitated with anti-P-Prp31 antibodies. (A) Northern blot analysis of the snRNA composition of endogenous spliceosomes. Spliceosomal complexes were purified from HeLa cells by using antibodies specific for the phosphopeptides P-SF3b155 (Bact) or P-Prp31 (B). For the control IP (CTRL) the antibody was omitted during the purification. Northern blotting was performed by using probes against the snRNAs, which have been radioactively labelled at the 3' end. (B) Northern blot analysis of the snRNA composition of endogenous spliceosomes stalled by siRNA-mediated depletion of Prp38, MFAP1, Smu1 or RED. 


\section{Discussion}

The spliceosome is a complex molecular machine that catalyses the removal of intronic sequences from a premRNA. While yeast spliceosomes are only comprised of the basic core spliceosomal proteins, spliceosomes in higher eukaryotes are equipped with numerous additional factors, likely to meet the requirements of a much more complex gene architecture and the resulting prevalence of alternative splicing. Two examples of proteins found in human but not yeast spliceosomes are Smu1 and RED. These so-called B-specific proteins transiently associate with the spliceosome at the B complex stage and are released/destabilized during spliceosome activation. While several other members of the B-specific proteins contribute to the activation of spliceosomal B complexes, the role of Smu1 and RED during pre-mRNA splicing is poorly understood. Previous studies have proposed roles for Smu1 and RED as mediators of spliceosomal protein interactions (Hegele et al., 2012), as alternative splicing regulators (Papasaikas et al., 2015), as well as factors that support the stability of the B complex (Bertram, Agafonov, Dybkov et al., 2017).

During my thesis I have investigated the function of Smu1 and RED in pre-mRNA splicing via a combination of genome wide, in vivo and in vitro approaches. My data revealed that Smu1 and RED are general splicing factors that enhance the B-to-Bact transition, and are essential for spliceosome activation when introns exhibit a critical distance between the 5'SS and the BS of $\sim 56 \mathrm{nt}$. The involvement of these proteins in an essential spliceosome assembly step indicates that Smu1 and RED are not only important for alternative splicing, but also function as general splicing factors that are required for productive and efficient activation of the human spliceosome.

\subsection{Efficient splicing is dependent on Smu1 and RED in vivo and in vitro}

Previous studies showed that knock-down of Smu1 or RED in human cells significantly changes alternative splicing, resulting in their classification as alternative splicing regulators (Spartz et al., 2004; Chung et al., 2009; Papasaikas et al., 2015). However, these studies did not address whether also constitutive splicing is affected by depletion of Smu1 and RED. To address this question, HeLa cells were siRNA-depleted of Smu1 or RED and splicing was analysed via RNAseq analysis (Fig. 3.1). Consistent with previous results, depletion of Smu1 or RED changed alternative splicing patterns significantly. Thereby, half of the altered splicing events were alternatively spliced cassette exons - which were preferentially skipped, while $30 \%$ involved alternative splice site selection (5'SS or 3'SS). Interestingly, more than $20 \%$ of the splicing events that were altered in Smu1 or RED-depleted cells represented retained introns. Importantly, these introns were predominantly constitutively spliced, suggesting that Smu1 and RED are important for splicing in general, and are not only involved in the regulation of alternative splicing. Importantly, cells depleted of Smu1 and RED showed striking overlaps in altered constitutive and alternative splicing events, but many of the altered splicing events were not affected by depletion of the B-specific protein MFAP1. This is consistent with the idea that Smu1 and RED form a functional module that acts independently of the other B-specific proteins. 


\section{Discussion}

A role for Smu1 and RED in constitutive splicing was also demonstrated in vitro for the MINX-120 pre-mRNA substrate, using HeLa nuclear extract that was immunodepleted of Smu1 and RED (Fig. 3.4). The efficiency of splicing was clearly less in the $\triangle$ Smu1/RED extract compared to the mock-depleted extract but splicing was not completely inhibited. Instead there appeared to be a slow-down in the rate of mRNA production when Smu1 and RED were missing. Investigation of spliceosome assembly on native agarose gels revealed a transient accumulation of spliceosomal B, but not A complexes, in the Smu1/RED-depleted extract with MINX-120, while the subsequently assembled $\mathrm{B}^{\mathrm{act}}$ and $\mathrm{C}$ complexes were still formed but at a slower rate. These results indicate that defects in splicing triggered by the absence of Smu1 and RED were specifically caused by impaired spliceosome activation, while the association and integration of the tri-snRNP were not affected. The slower/less efficient activation thus appears to be the basis for the slower pre-mRNA-to-mRNA conversion. The effect of the Smu1/REDdepletion on in vitro splicing was not substrate-specific as other pre-mRNAs, with a very different architecture such as PM5, were also spliced less efficiently in the absence of Smu1/RED, while B complexes temporarily accumulated (Fig. 3.9).

An effect on the kinetics of spliceosome activation could also explain how, at least in some cases, Smu1 and RED knock-down affect alternative splicing in vivo. Similar to the elongation rate of the RNA polymerase II, which is thought to be an important factor for the regulation of alternative splicing (de la Mata et al., 2003; Dujardin et al., 2014), a delay in spliceosome activation might be rate determining during splicing and consequently favour the selection of weaker splice sites. Thus, the changes in alternative splicing observed by RNA-seq analyses in Smu1and RED-depleted cells may be due to a generally slowed-down spliceosome assembly.

\subsection{Evidence that knock-down of Smu1 and RED also hinders spliceosome activation in vivo}

To provide evidence that Smu1 and RED are also involved in spliceosome activation in vivo, I utilised phosphopeptide-specific antibodies against SF3b155 and Prp31 (Fig. 3.17). B complex formation depends on phosphorylation of Prp31 (Schneider, Hsiao et al., 2010), which is subsequently released with U4 during spliceosome activation. Thus, only assembled B complexes harbour the Prp31 phosphoprotein. SF3b155 is hyperphosphorylated first during the activation step and its dephosphorlyation is essential for the second catalytic step of splicing (Shi et al., 2006; Girard et al., 2012). Thus, phosphorylated SF3b155 is specifically associated with catalytically activated spliceosomes. As a positive control to confirm that these antibodies can be used to detect changes in B and $\mathrm{B}^{\text {act }}$ complex formation in vivo, the B-specific proteins Prp38 and MFAP1 were knocked-down in HeLa cells (Fig. 3.18). The depleted cells were fractionated into a nucleoplasmic and a chromatin fraction and analysed by western blotting. Prp38 has been shown in yeast and human (Xie et al., 1998; Schütze et al., 2016) to be involved in spliceosome activation and Spp381, the yeast counterpart of MFAP1, appears to interact with yPrp38 thereby contributing to its function during the activation (Lybarger et al., 1999; Ulrich \& Wahl, 2017). Most likely, MFAP1 likewise supports Prp38 function in humans. 
Indeed, Prp38- and MFAP1-depletion in HeLa cells led to an increased signal of P-Prp31 in the chromatin fraction. As splicing occurs predominantly co-transcriptionally in the cell, active spliceosomes are mainly associated with this fraction (Pandya-Jones \& Black, 2009; Girard et al., 2012). Concomitant with the increased P-Prp31 signal, chromatin-associated P-SF3b155 appeared to be reduced. This indicates that knock-down of Prp38 or MFAP1, results in accumulation of $B$ complexes on the chromatin at the expense of $B^{\text {act }}$ complex formation. As these changes are consistent with impaired spliceosome activation, this result supports the idea that P-Prp31 and PSF3b155 are good markers for the formation of B and $B^{\text {act }}$ spliceosomes in the cell. As the investigation of spliceosome assembly in vivo is challenging, the availability of tools like these phospho-specific antibodies is extremely helpful and can potentially be extended to additional assembly intermediates to allow a better overall picture of spliceosome assembly in vivo.

To further demonstrate the specificity of P-Prp31 and P-SF3b155 for assembled B and Bact complexes, respectively, I purified the spliceosomal particles that contain the respective phoshopeptide using a method similar to chromatinimmunoprecipitation (Fig. 3.19). This procedure includes protein-protein crosslinking to maintain the integrity of the complexes during the subsequent sonication step, which fragments the nucleic acids resulting in the separation of individual spliceosomal complexes. While the P-Prp31-specific antibody precipitated complexes containing all five snRNAs, U1 and U4 were significantly reduced in spliceosomes containing P-SF3b155 (Fig. 3.20A). Although the formation of a $B$ complex essentially requires the destabilization/release of the U1 snRNP, P-Prp31 containing spliceosomes are nonetheless likely pre-catalytic B complexes and the strong abundance of U1 appears to be an artefact of the purification method. U1 snRNPs might remain loosely associated with the spliceosome after its removal from the 5'SS and be covalently attached due to the cross-linking step. Alternatively, as U1 is the most abundant snRNP in the nucleus of mammalian cells (Baserga \& Steitz, 1993) and the first to be recruited to the pre-mRNA, it is possible that $\mathrm{U} 1$ is bound to 5' splice sites upstream or downstream of the intron, thereby increasing the apparent abundance of the U1 snRNA in the P-Prp31 containing spliceosomes. Nevertheless, P-Prp31 and PSF3b155 are clearly associated with assembled spliceosomes, which exhibit snRNA profiles consistent with them being $\mathrm{B}$ and $\mathrm{B}^{\text {act }}$ complexes, respectively.

To examine whether the changes in constitutive splicing in $\triangle S m u 1$ and $\Delta R E D$ cells were likewise likely caused by defective spliceosome activation, I performed RNAi-mediated knockdowns of Smu1 and RED in Hela cells and followed the effects on spliceosome activation using the anti-P-Prp31 and P-SF3b155 antibodies. Interestingly, knockdown of Smu1 or RED led to a strong co-depletion of the respective other protein. This supports the idea that the stability of each protein is improved when they are able to interact with one another (Spartz et al., 2004). It further indicates that the effects observed in previous in vivo studies reflect the combined effect of the depletion of both proteins. Investigation of endogenous spliceosome assembly by western blotting revealed an increase in the P-Prp31 signal and a decrease in the P-SF3b155 signal in $\triangle$ Smu1 and $\triangle$ RED cells, similar to what was observed for Prp38- and MFAP1-depleted cells. This indicates that the activation of the spliceosome is also impaired in vivo in the absence of Smu1 and RED. 


\section{Discussion}

\subsection{The majority of Smu1 and RED exist as a unit in HeLa cells}

Immunodepletion of Smu1 from splicing-competent nuclear extract, using a well-functioning Smu1-specific antibody, led to the near quantitative removal of Smu1 and additionally more than $90 \%$ of RED, indicating that the two proteins physically interact in extract. The Smu1-RED interaction appears to be highly stable, as it resisted salt concentrations up to $1 \mathrm{M} \mathrm{KCl}$ (data not shown). Smu1 and RED have previously been anticipated to form a functional module based on functional similarities between these two proteins (Papasaikas et al., 2015; Ulrich et al., 2016). This conclusion was also supported by biochemical techniques to identify protein-protein interactions, such as the yeast two-hybrid system $(\mathrm{Y} 2 \mathrm{H})$, which suggested that Smu1 and RED are able to physically interact (Hegele et al., 2012). Binding studies with Smu1 and RED from Chaenorhabditis elegans, which are highly homologous to the human Smu1 and RED proteins, also demonstrated that these proteins interact and regions of the proteins minimally required for stable interaction were defined (Ulrich et al., 2016). The highly stable and specific codepletion of the vast majority of RED with Smu1 provides additional convincing evidence that the vast majority of human Smu1 and RED naturally exist as a unit in HeLa cells (Fig. 3.3). Recent cryo-EM studies of the human B complex revealed that Smu1 and RED interact within the spliceosome (Bertram, Agafonov, Dybkov et al., 2017). The C-terminal WD40 domain and the N-terminal region (NTR) of Smu1 could be localized in the B complex together with a short alpha helix of RED (aa 209-222) that was shown previously to interact with the NTR of Smu1 in C. elegans (Ulrich et al., 2016), indicating that Smu1 and RED retain their dimer status even after they are integrated into the spliceosome (Fig. 4.1).

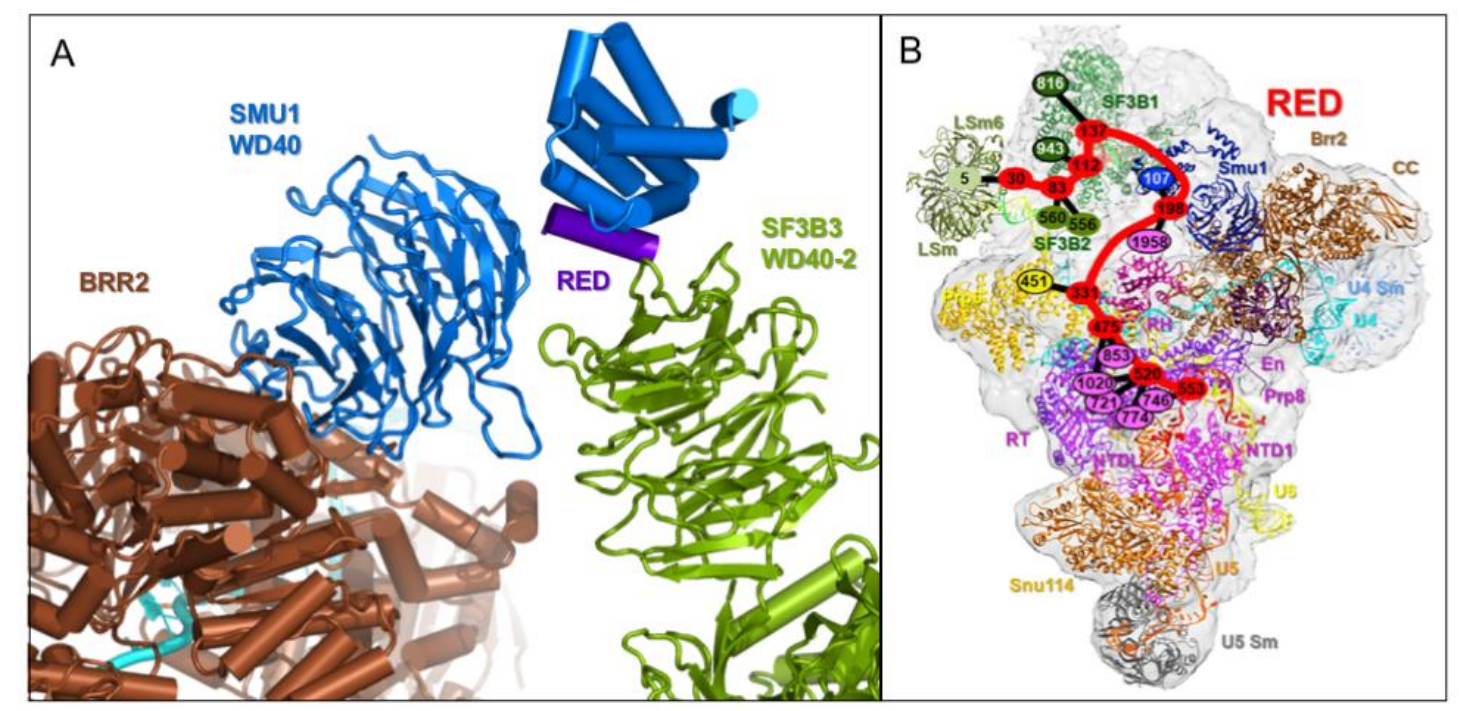

Figure 4.1. Location of Smu1 and RED within the human B complex. (A) Locations of the C-terminal WD40 domain and the N-terminal region (NTR) of Smu1 at the interface between the U5-assocated helicase Brr2 and U2 protein SF3B3. The short alpha helix of RED (aa 209-222) that interacts with the NTR of Smu1 is shown in purple. (B) Overview of the likely location of RED based on cross-linking experiments. The anticipated path of RED is indicated as a red line, while the colored ovals with numbers indicate the intermolecular crosslinks between RED and other spliceosomal factors. The illustration was taken from Bertram, Agafonov, Dybkov et al., 2017. 


\subsection{The interaction between Smu1 and RED is essential for their spliceosomal association and function}

Depletion of Smu1 and RED in vitro suggested that nearly the entire populations of these proteins exist as a functional unit in the cell. According to the cryo-EM structure of the human B complex, this interaction is maintained after association of Smu1 and RED with the spliceosome, suggesting that it might be important for their mutual function (Bertram, Agafonov, Dybkov et al., 2017). To investigate whether Smu1 and RED need to interact to support the activation of the spliceosome or whether one of these proteins could fulfil this function on their own, Smu1 and RED were individually expressed in insect cells and added to the $\triangle$ Smu1/RED extract (Fig. 3.12). Addition of the single proteins did not substantially restore the B-to-B ${ }^{\text {act }}$ transition or splicing product formation. However, when the two proteins were added together, B complexes no longer accumulated, and $\mathrm{B}^{\text {act }}$ and $\mathrm{C}$ complexes were formed. This shows that Smu1 and RED do not need to be co-expressed to form a functional module, and indicates that Smu1 and RED do not function on their own.

As Smu1 and RED interact in HeLa nuclear extract before their association with the spliceosome, it is possible that their interaction not only directly affects their function, but may be required for their efficient incorporation into the spliceosome. Consistent with this idea, B complexes formed after addition of Smu1 or RED to $\triangle$ Smu1/RED splicing extract contained only low levels of each protein, whereas the association of each protein was strongly enhanced when both proteins were added together (Fig. 3.13). This shows that Smu1 and RED bind poorly or less stably to the spliceosome as individual proteins. Their interaction might introduce important structural changes in Smu1 and RED that enable their proper/stable interaction with other B complex-associated spliceosomal components. Interestingly, the NTR domain of Smu1 and the short helix of RED that are required for dimerization are localized in close proximity to the Smu1-WD40 domain at the SF3B3-Brr2 interface (Fig.4.1A). It is possible that Smu1 requires RED to adopt this conformation, which is a prerequisite for it to simultaneously contact SF3B3 and Brr2, and thus to be stably incorporated into the B complex. Indeed, crystallography studies of Smu1 and RED from $C$. elegans, showed that Smu1 undergoes global conformational changes upon RED binding (Ulrich et al., 2016). Taken together these results show that Smu1 and RED do not bind efficiently or productively to the spliceosome on their own nor function independently of one another.

\subsection{Dissection of regions of Smu1 and RED required for their function in splicing}

Smu1 contains a C-terminal WD40 domain, which is known to mediate protein-protein interactions. Its $\mathrm{N}$-terminal alpha-helical domain interacts with RED, which is intrinsically disordered, but forms two short helices in the region where it interacts with Smu1. The position of the Smu1-WD40 domain at the U2/tri-snRNP interface in the B complex (Fig. 4.1A) suggests an important role for it in mediating the association of Smu1 and RED with the spliceosome. When full-length RED was co-expressed with a Smu1-WD40 truncation mutant, the dimer could be purified suggesting that the Smu1/RED interaction was not impaired by the absence of the WD40 domain. 


\section{Discussion}

Addition of the Smu1-AWD40/RED dimer to Smu1/RED-depleted extract was not able to restore spliceosome activation, suggesting that this domain is important for the function of the entire dimer (Fig. 3.14) However, analysis of $\mathrm{B}$ complexes assembled in the presence of the Smu1- $\mathrm{WWD40/RED} \mathrm{dimer} \mathrm{revealed} \mathrm{that} \mathrm{the} \mathrm{dimer} \mathrm{was} \mathrm{not}$ associated with these complexes, indicating that the WD40 domain is essential for proper interaction of the dimer with the spliceosome. As spliceosome binding was impaired, the role of the WD40 domain in spliceosome activation and splicing catalysis could not be investigated.

To assess the structural requirements of RED for the function of the Smu1/RED dimer, RED was N- or C-terminally truncated, but its region required for stable interaction with Smu1 was retained. Based on crosslinking experiments with purified human B complexes (Bertram, Agafonov, Dybkov et al., 2017), RED's N-terminal region interacts with the U2 proteins SF3B1 (SF3b155) and SF3B3, while its C-terminus contacts Prp8 at several positions (Fig. 4.1B). Consequently, these truncations could potentially reduce the stability of the interaction of RED with the spliceosome. Addition of the $\triangle \mathrm{N}-\mathrm{RED}$ or RED- $\Delta \mathrm{C}$ mutants together with full-length Smu1 to $\Delta$ Smu1/RED extract allowed spliceosome activation, but the latter was strongly reduced when both termini were removed (RED- $\Delta \Delta)$ (Fig. 3.15). Thus, RED is - to a certain extent - still able to fulfil its function as long as only one interaction surface was removed. This suggests that the contacts RED establishes with U2 or U5 individually are not essential for the function of Smu1/RED, at least not for the splicing of the MINX-80 substrate.

\subsection{Smu1 and RED do not indirectly affect activation by acting as a binding platform for other B complex proteins}

I could show that Smu1 and RED contribute to efficient activation of the spliceosome and thus the question arises whether these proteins affect the activation directly or indirectly and by what mechanism. Investigation of the protein-protein interaction network in the spliceosome by $\mathrm{Y} 2 \mathrm{H}$ experiments suggested that Smu1 and RED directly or indirectly contact numerous spliceosomal proteins (Hegele et al., 2012). These interactions included MFAP1, as well as single components of the U5 snRNP, the U4/U6 di-snRNP, the RES complex, the IBC and the Prp19-related proteins. Protein-protein crosslinking with purified human B complexes also indicated that RED meanders through the spliceosome and contacts multiple spliceosomal proteins (Fig. 4.1B). Therefore, both proteins were proposed to act as binding platforms for additional spliceosomal proteins. Depletion of Smu1 and RED could thus potentially alter the binding of other $B$ complex proteins and this could in turn compromise the efficiency of the activation process. Interestingly, B complexes assembled in Smu1/RED-depleted extract sedimented differently in glycerolgradients compared to B complexes assembled in wildtype extracts, peaking one or two fractions closer to the top of the gradient. This slower migration can be explained by a lower molecular weight (e.g. if the complex contains fewer proteins) or by a less compact conformation (Fig. 3.8A). 
With the exception of the absence of Smu1 and RED, the composition of the $\triangle$ Smu1/RED B complexes was essentially identical to B complexes assembled in mock-depleted extract. Both contained stoichiometric amounts of U2, U4, U5 and U6, while U1 was nearly absent (Fig. 3.8B). This is consistent with the composition of stable B complexes formed after the action of Prp28, which leads to U1 displacement, but before Brr2-driven unwinding of the U4/U6 duplex.

All known B complex proteins were present including U2 and tri-snRNP proteins, as well as the remaining set of Bspecific proteins (Table 3.1). Importantly, factors known to be required for the activation of B complexes, such as Prp38 or FBP21, were clearly present. This demonstrates that their recruitment is independent of Smu1/RED and that the stalling at the $\mathrm{B}$ complex level is not caused by the absence of these functionally important proteins.

Some differences in the protein composition of the wildtype versus $\Delta$ Smu1/RED B complexes could be observed for proteins known to be recruited during/after activation such as the Prp19/CDC5L complex, the IBC or Bact and C specific proteins, which were essentially absent in the $\triangle$ Smu1/RED B complexes. These proteins are often found to a minor extent in kinetically stalled B complexes but are absent from B complexes stalled with ATP $\gamma$ S or by a low $\mathrm{MgCl}_{2}$ concentration (Agafonov, Van Santen et al., 2016; Bertram, Agafonov, Dybkov et al., 2017). This is likely due to the more homogenous composition of the latter B complex preparations, while kinetically stalled B complex preparations include low levels of activated spliceosomes. Taken together the snRNA and protein compositions of the B complexes stalled by the absence of Smu1/RED are consistent with assembly being stalled after Prp28promoted release of $\mathrm{U} 1$ but before Brr2-catalyzed unwinding of $\mathrm{U} 4$ and $\mathrm{U} 6$. As no factors known to be required for spliceosome activation were missing, it appears that Smu1 and RED play a direct role in the activation process.

However, the aberrant sedimentation behaviour of the $\triangle$ Smu1/RED B complexes suggests that the absence of these proteins leads to structural changes in the spliceosome, in particular a less compact conformation. Thus, it is still conceivable that the absence of Smu1 and RED may still indirectly affect activation by changing the interactions within the spliceosome involving other proteins such as Prp38 or FBP21. Smu1 and RED form a bridge between the U2 SF3B3 protein and Brr2 in the B complex (Fig. 4.1). First structural investigations of $\triangle$ Smu1/RED B complexes by negative staining electron microscopy, revealed a structure highly similar to that of DeltaMgCl $2 \mathrm{~B}^{2}$ complexes and no extended gap between U2 and Brr2 was visible in the absence of Smu1/RED. Cautiously speaking, U2 and especially Brr2 appeared slightly fuzzier in B complexes lacking Smu1 and RED, but the low resolution of this method prohibits drawing solid conclusions. Future cryo-EM of $\Delta$ Smu1/RED B complexes may provide sufficient resolution to allow insight into any structural changes due to the absence of these proteins. 


\section{Discussion}

\subsection{Smu 1 and RED might directly affect Brr2 helicase activity during the earliest step of activation}

Spliceosome activation is a multi-step process, which is initiated by unwinding of the U4/U6 duplex by the RNA helicase Brr2, which is a stable U5 snRNP component. This allows U6 to rearrange and also to interact with U2, which forms the catalytically active U2/U6 RNA structures in the spliceosome. This also leads to loss of U4 and U4/U6 proteins and also the B-specific proteins. Further, the Prp19/CDC5L complex and related proteins, IBC and

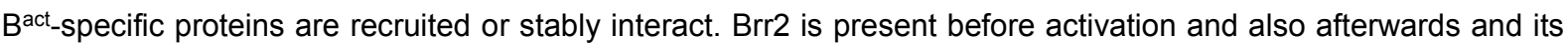
activity thus must be tightly regulated to prevent premature activation. Several mechanisms repress Brr2 before and after activation and its RNA unwinding activity must be triggered in a currently unknown way to start the activation process (Absmeier et al., 2016). Smu1 and RED bridge the U2 SF3B3 protein and Brr2 in the B complex (Fig. 4.1), with Smu1 directly contacting the N-terminal and C-terminal helicase cassettes of Brr2. Thus, these proteins could contribute to positioning Brr2 relative to its substrate in a way that promotes U4/U6 unwinding. Consistent with this idea, Brr2 is very flexible and undergoes a large-scale movement from its position in the trisnRNP to its position close to U4/U6 in the B complex (Bertram, Agafonov, Dybkov et al., 2017), and Smu1 and RED could help to anchor Brr2 in this position. Alternatively or additionally, Smu1 could help in triggering Brr2 helicase activity by its direct interaction with the helicase cassettes via a mechanism that is currently not clear.

\subsection{Smu1 and RED are essential for the activation of spliceosomes formed on very short introns}

Although the majority of introns found in human cells are much longer than $200 \mathrm{nt}$, introns retained after knockdown of Smu1 or RED were predominantly shorter, with most between 75 and 100 nt long (Fig. 3.2). As this preference was not observed after knockdown of MFAP1, Smu1 and RED appear to be specifically required for the splicing of very short introns. Nevertheless, a substantial fraction (30-40\%) of the retained introns were significantly longer, consistent with a general requirement for Smu1 and RED for efficient splicing also of longer introns. In vitro splicing studies using truncated versions of the MINX-120 pre-mRNA, also demonstrated that splicing is more dependent on the presence of Smu1 and RED when intron length was shorter (Fig. 3.6). That is, I performed splicing in mock-depleted or Smu1/RED immunodepleted HeLa nuclear extract and compared the splicing of the standard MINX pre-mRNA substrate - which contains a 120 nt long intron - with two truncated versions and one elongated variant. In these the intron was shortened or extended between the 5'SS and the BS, changing the overall intron length from $120 \mathrm{nt}$ to 90 or $80 \mathrm{nt}$, or to $150 \mathrm{nt}$, respectively. Splicing of MINX-120 and MINX-150 was less efficient in the absence of Smu1/RED compared to the mock-depleted extract, but no increase in splicing efficiency was detected with MINX-150. As extending the intron did not have a significant effect on splicing, $120 \mathrm{nt}$ appears to be above the critical length needed to observe enhanced dependency on Smu1/RED. 
Importantly, spliceosome activation was not only delayed but completely blocked when introns were very short. Truncation of the intron to either 90 or 80 nt reduced the overall efficiency of splicing compared to MINX-120, but it also enhanced the inhibitory effect of Smu1/RED depletion. While MINX-90 was spliced somewhat less efficiently than MINX-120, splicing of MINX-80 was nearly abolished in the absence of Smu1 and RED. Analysis of splicing complex formation on MINX-80 showed a strong accumulation of B complexes with little or no Bact or catalyticallyactive C complexes formed when Smu1 and RED were absent. Splicing and spliceosome activation of MINX-80 could be restored to mock levels by addition of the purified dimer to the depleted extract, demonstrating that the observed inhibitory effect was solely due to the absence of Smu1 and RED and not due to the co-depletion of any other essential splicing factors. Thus, both in vitro and in vivo Smu1 and RED play a crucial role in the splicing of extremely short introns.

\subsection{The distance between the 5'SS and the BS is critical for splicing and determines whether Smu1 and RED are essential}

In MINX-90, -80 and -70, the distance from the BS-A to the 5'SS was shortened from $96 \mathrm{nt}$, to 66, 56 and 46 nts, respectively, and there was a progessive loss of splicing activity in the mock-depleted extract, with splicing completely blocked with MINX-70. This is consistent with previous studies showing that a minimum 5'SS-BS distance is critical for splicing (Ruskin et al., 1985; Fu et al., 1988; Köhrer \& Domdey, 1988; Smith \& Nadal-Ginard, 1989; Himmelspach et al., 1991). With MINX-80, impaired spliceosome activation was seen under standard conditions, but the B-to-B ${ }^{\text {act }}$ transition was completely prevented in the absence of Smu1 and RED. Interestingly, spliceosome activation and splicing were also entirely blocked when the 5'SS-BS distance was truncated from 150 to $56 \mathrm{nt}$ in the PM556 and PM5-1056 pre-mRNAs (Fig. 3.9). While the intron of the PM5-1056 pre-mRNA was with 73 nt shorter than the MINX-80 intron, the PM556 intron was with 142 nt considerably longer. As spliceosome assembly was similarly impaired with both pre-mRNAs, the 5'SS-BS distance rather than the overall intron length appears to be the decisive factor for the Smu1/RED-dependence. Additionally, spliceosome assembly on both PM556 and PM5$10_{56}$ was equally blocked at B complex level. As they only differ in the length of their PY tracts, the distance between BS and 3'SS does not appear to have an impact on whether or not Smu1 and RED are essential. Taken together these results indicate that the distance between the 5'SS and the BS rather than the BS-3'SS distance or intron length per se is the decisive factor for the Smu1/RED-dependency during short intron splicing.

In the human B complex, a $17 \mathrm{nt}$ long extended helix is formed between U6 (i.e. the U6 ACAGAG box and adjacent nucleotides) and nucleotides within the intron near the 5'SS (Fig. 4.2A). Similarly, the BS forms a 14 nt long helical duplex with the U2 snRNA (Fig. 4.2B). (Bertram, Agafonov, Liu et al., 2017; Haselbach et al., 2018; X. Zhang et al., 2018). In B complexes formed on MINX-120 pre-mRNA, these two extended helices are separated by $15 \mathrm{~nm}$ (Bertram, Agafonov, Dybkov et al., 2017), which corresponds to 21 nt of RNA in an extended conformation (Fig. $4.2 C)$. 


\section{Discussion}

Consequently, to span the distance between the U6 ACAGAG/5'SS helix and the U2/BS helix, without the need for a substantial conformational change in the spliceosome, a minimum of $\sim 52 \mathrm{nt}$ would be necessary between the 5'SS and the BS (Fig. 4.2C). This is approximately the distance found in MINX-80, PM556 and PM5-1056 suggesting that the intron nucleotides in this region are likely in a fully extended conformation in the B complex. This could potentially limit the flexibility of the spliceosome during its remodelling events, thereby impeding or - in the absence of Smu1/RED - completely preventing its activation.

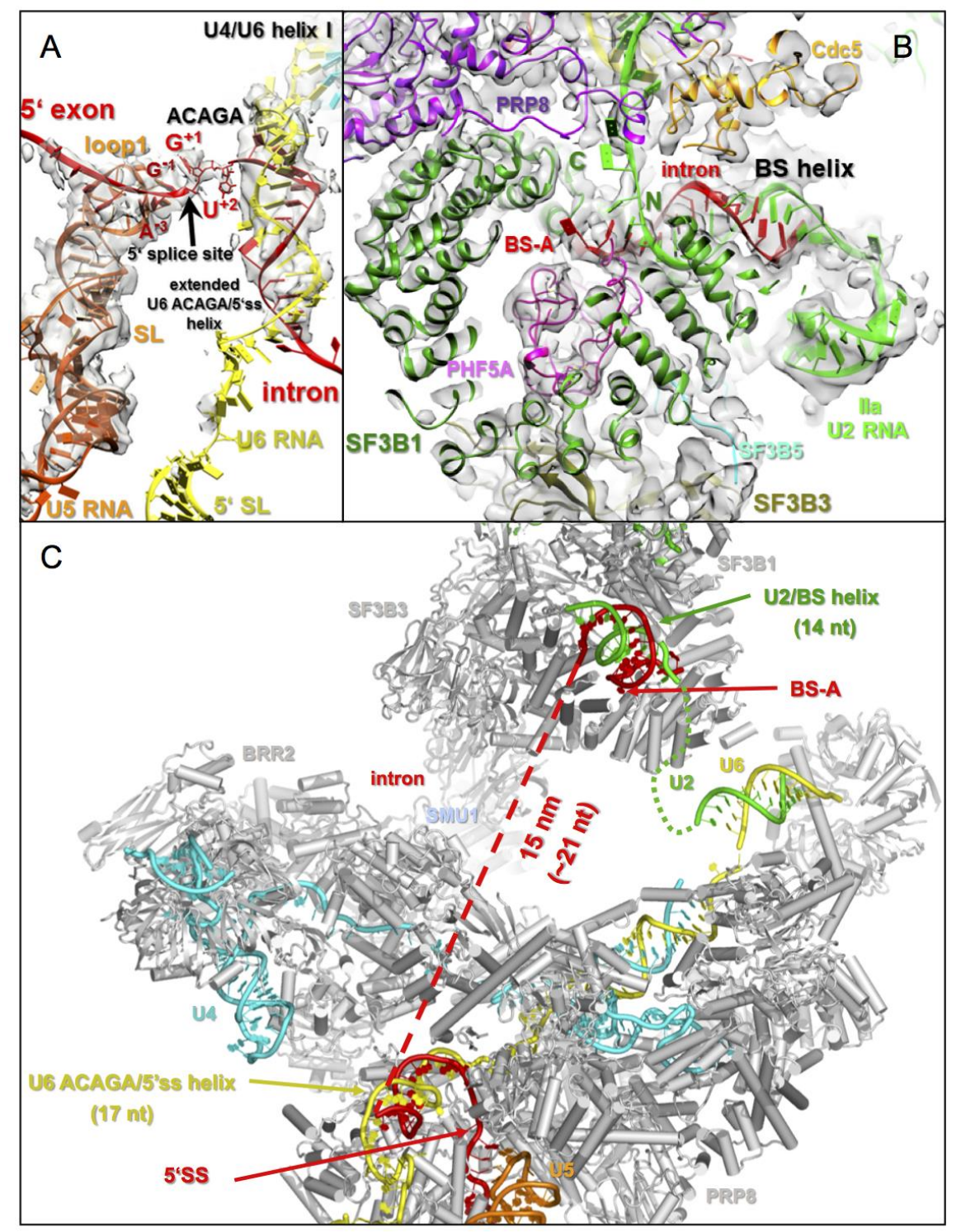

Figure 4.2. Location and interactions of the pre-mRNA within the human B complex. The intron forms extended helices with the U6 ACAGAG box and adjacent U6 nucleotides at the 5'SS (A) and the U2 snRNA at the BS (B). The two helices are separated by $15 \mathrm{~nm}$ $(\sim 21)$ in the human B complex. This would accommodate an estimated minimum of 54 nt between the 5 'SS and the BS, without altering the structure of the spliceosome. These structures were kindly provided my Berthold Kastner, Department of Cellular Biochemistry, MPIBPC. 


\subsection{A short 5'SS-BS distance represents a steric hindrance for the spliceosome}

Previously, the requirement for a minimal 5'SS-BS distance was explained by steric hindrances that prevent the binding of the large snRNPs. The dependence of spliceosome activation on a minimal 5'SS-BS distance is probably due to physical limitations exerted by the intron (Fig. 4.2). In this case, resolving this steric hindrance should allow spliceosome activation even without the support of Smu1 and RED. Indeed, when the MINX-80 pre-mRNA was cleaved into two RNAs, spliceosome activation was restored both in the presence or absence of Smu1/RED (Fig 3.10). Specifically, while the intact MINX-80 resulted in a strong accumulation of B complexes, the cleaved substrate allowed the formation of activated spliceosomes and $\mathrm{C}$ complexes indicating that the activation was no longer inhibited. Thus, physically separating the 5'SS and the BS to release any length limitations restores the conversion of $B$ to $B^{\text {act }}$ complexes.

\subsection{Model for how the 5'SS-BS distance affects the dependency of spliceosome activation on Smu1/RED}

Taking into consideration my data and utilizing the structural information obtained from the cryo-EM structure of the human B complex, a model for how 5'SS-BS distance might lead to the dependency of spliceosome activation on Smu1/RED was generated (Fig. 4.3). In the human B complex Smu1 and RED form a molecular bridge between the U2 and tri-snRNPs (Bertram, Agafonov, Dybkov et al., 2017). In the yeast spliceosome where Smu1 and RED do not exist, this bridge is formed directly by the Brr2 and SF3B3 homologs (Plaschka et al., 2017). Here, a WD40 domain of Rse1, the yeast counterpart of SF3B3, is positioned between the two cassettes of Brr2, which is replaced by the WD40 domain of Smu1 in the human B complex. This interaction likely stabilizes the position of Brr2 relative to its U4/U6 substrate or might even directly stimulate Brr2 activity thereby promoting activation (see above). Plaschka et al. described the Rse1-Brr2 interaction in yeast as transient and suggested that U2 and Brr2 might need to be able to move independently from each other during spliceosome activation. In contrast, my data suggest that the stability of this bridge is important. It is possible that the apparent transience of this bridge in the yeast spliceosome rather represents two distinct states; B complexes before and after establishment of the Rse1-Brr2 interaction. Alternatively, this bridge could be less important in yeast.

In the human spliceosome, Smu1 and RED could ensure that the contact between U2 and Brr2 is established faster and remains stable during activation. As longer introns allow spliceosome activation in the absence of Smu1 and RED, but with reduced efficiency, it appears that a sufficiently long intron grants the U2 head domain enough flexibility to move towards Brr2, coincidentally forming the apparently essential SF3B3-Brr2 bridge, only at a slower pace (Fig. 4.3A). In contrast, an intron with a critical 5'SS-BS distance of $\sim 56 \mathrm{nt}$ would be completely extended in between those nucleotides forming a duplex with U2 or U6, and therefore physically restrict the mobility of U2. This would prevent the movements necessary for U2-SF3B3 to contact Brr2 (Fig. 4.3B) and this would be most severe when Smu1 and RED are absent. In this case, physically separating the 5'SS from the BS would release this 


\section{Discussion}

physical constraint. Consequently, the flexibility of U2 that allows it to move towards Brr2 would be restored, allowing spliceosome activation even without the support of Smu1 and RED.
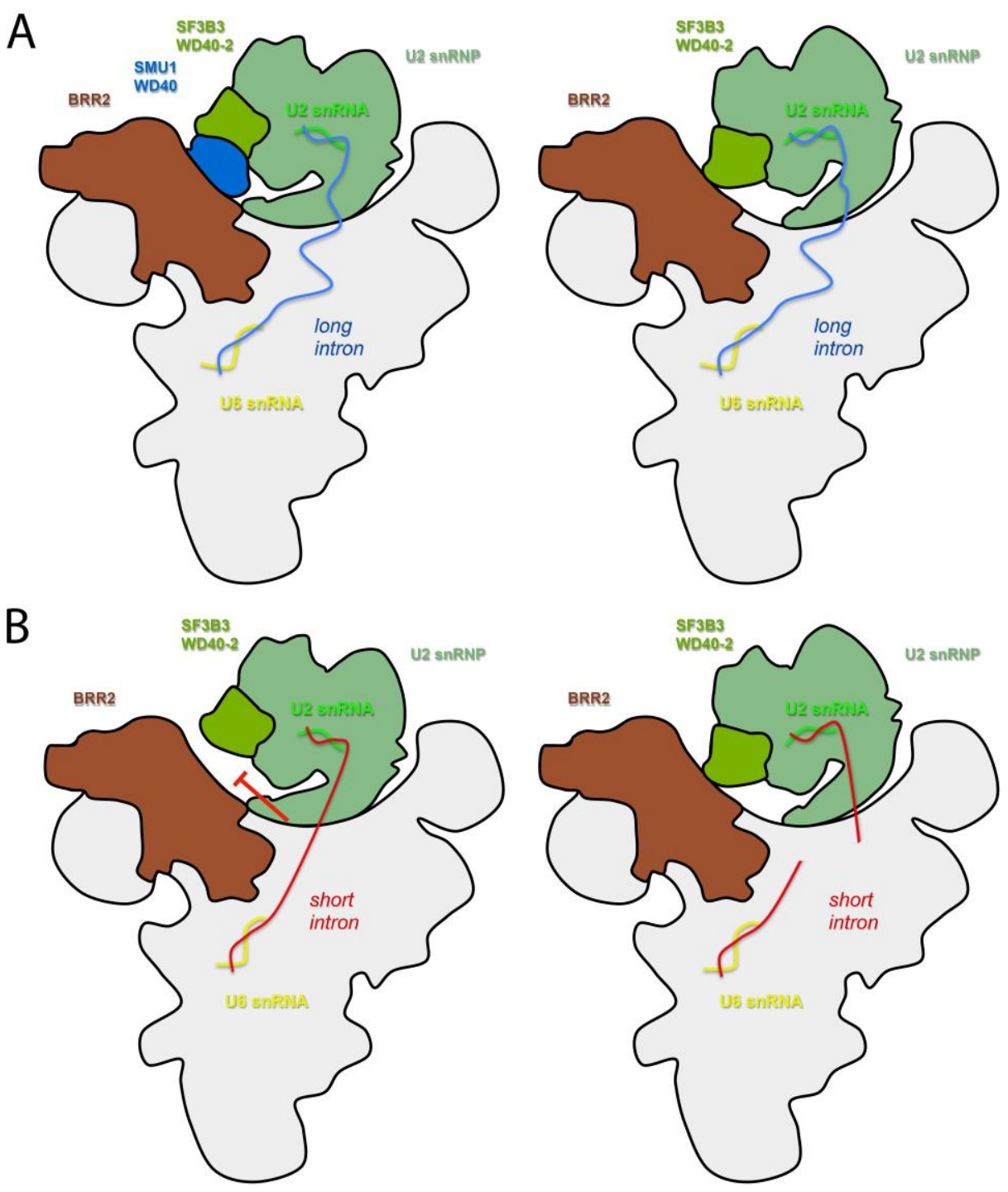

Figure 4.3. Model for how the 5'SS-BS distance affects the dependency of spliceosome activation on Smu1/RED. (A) On long introns Smu1 supports/stabilizes the formation of the Brr2-U2 bridge, which is likely important for spliceosome activation. In the absence of Smu1/RED, this bridge can still be formed, albeit at a slower rate, as long as the intron's distance between the 5 'ss and BS is sufficiently long to allow movement of the U2 domain towards Brr2, resulting in a direct Brr2-U2 interaction. (B) On very short introns, with a short distance between the 5'SS and the BS, the U2 snRNP may be repositioned and/or its ability to move towards Brr2 may be inhibited due to the structural constraint of the intron. Therefore, no direct contact between U2 and Brr2 can be established, and Smu1 and RED become essential for activation. Splitting the MINX-80 pre-mRNA into two pieces may relieve this constraint, allowing the B-to-Bact transition even in the absence of Smu1 and RED. 


\subsection{The tri-snRNP still binds the MINX-70 pre-mRNA}

Previous studies indicated that the binding of snRNPs to the pre-mRNA was abolished when the 5'SS-BS distance was too short (Ruskin et al., 1985; Fu et al., 1988; Köhrer \& Domdey, 1988; Smith \& Nadal-Ginard, 1989; Himmelspach et al., 1991). For example, binding of U2 was prevented when the distance between the 5'SS and the BS was extremely short - e.g. less than $35 \mathrm{nt}$ in the case of an adenovirus pre-mRNA - and stable tri-snRNP binding (i.e., B complex formation) was abolished when this distance was less than $50 \mathrm{nt}$ (Himmelspach et al., 1991). Recent cryo-EM studies of the human B complex provide a structural basis for this distance dependency. As mentioned above, the structure of a functional B complex formed on MINX-120 would likely not require substantial rearrangement/changes if the 5 'SS/BS distance is above ca. $50 \mathrm{nt}$, but substantial changes, that are likely inhibitory, would occur if this distance is much shorter (Fig. 4.2C).

In contrast to previous studies, my results show that tri-snRNP binding can still occur if this distance is less than 50 $\mathrm{nt}$, and further show that the subsequent remodelling of the spliceosme during activation is affected when the distance between the 5'SS and the BS is short. That is, investigation of the spliceosome assembly on MINX-70 by native gel electrophoresis indicated a strong accumulation of $A$ complexes, but also indicated that $B$ complexes were still substantially formed (Fig. 3.5). A more detailed characterization of the spliceosomes formed on MINX-70 showed that their sedimentation behaviour on glycerol gradients was identical to that of $B$ complexes formed on MINX-120 and MINX-80 pre-mRNA (Fig. 3.16). Their protein compositions were also highly similar, with the presence of B-specific proteins in all cases, consistent with B complexes being assembled on MINX-70 (Table 3.2).

A notable difference was the presence of substantially more U1 snRNA in the MINX-70 B complexes, compared to MINX-120 or MINX-80 B complexes (Fig. 3.16). During spliceosome assembly the tri-snRNP first associates loosely with the A complex to form the pre-B complex (Boesler et al., 2016). In pre-B complexes U1 is stoichiometrically present and base pairs with the 5'SS. Stable tri-snRNP binding is then observed after U1 is displaced from the 5'SS and the U6 ACAGA box sequence base pairs with the 5'SS. This replacement of U1 by U6 is catalysed by the Prp28 (U5-100K) RNA helicase (Staley \& Guthrie, 1999). Thus, stable B complexes normally contain very low levels of $\mathrm{U} 1$, which probably are not, base-paired with the 5'SS but instead are still associated via protein-protein contacts with the B complex. The enrichment of the U1 snRNA in MINX-70 B complexes is reminiscent of the situation with pre-B complexes. However, it has been shown that the B-specific proteins, which are present in the particles assembled on MINX-70, are not normally recruited before the action of Prp28. It is thus possible that MINX-70 allows the formation of an intermediate spliceosome between the pre-B and $\mathrm{B}$ complexes, where an aberrant structure caused by the short 5'SS to BS distance impairs Prp28 action but still allows relatively stable binding of the tri-snRNP. Interestingly, Prp28 (U5-100K) was clearly enriched in the MINX-70 spliceosomes, which suggests that it may not have acted and removed $\mathrm{U} 1$ from the 5'SS. 


\section{Discussion}

Future RNA-RNA crosslinking studies should clarify if U1 still base pairs with the 5'SS in the MINX-70 B-like complexes or if the U6 ACAGAG box duplex is formed. An alternative explanation would be that a different structural organization of the MINX-70 complexes results in stabler U1 binding via protein-protein contacts alone. Future cyroEM of spliceosomes formed on MINX-70 might reveal changes in their structure due to the very short intron.

\subsection{Perspectives}

The aim of this thesis was to clarify the role of the non-conserved, B-specific proteins Smu1 and RED. I could show that Smu1 and RED are involved in the Brr2-driven activation of the spliceosome in vitro and in vivo. In the majority of cases these two proteins facilitate/accelerate the B-to-Bact transition, but when the distance between the 5'SS and the BS reaches a critical range of around $56 \mathrm{nt} \mathrm{Smu1} \mathrm{and} \mathrm{RED} \mathrm{are} \mathrm{essential.} \mathrm{The} \mathrm{cryo-EM} \mathrm{structure} \mathrm{of} \mathrm{the}$ human B complex revealed that Smu1, via its WD40 domain, bridges the U2-tri-snRNP interaction by simultaneously binding to SF3B3 and Brr2 (Bertram, Agafonov, Dybkov et al., 2017). Possibly, this interaction, which is formed directly in the yeast spliceosome (Plaschka et al., 2017), positions Brr2 properly relative to its U4/U6 substrate, but it might also directly stimulate the helicase activity of Brr2. This could be tested in the future by using a helicase assay, where the unwinding efficiency of the U4/U6 duplex is monitored in the presence or absence of Smu1 and RED. Similar experiments conducted with Brr2 and fragments of FBP21 indicated that FBP21 decreases the unwinding efficiency of the helicase in vitro (Henning et al., 2017) and could thus help to prevent pre-mature activation in spliceosomes primed for activation. For Smu1 and RED this type of experiment would provide information about the mechanism whereby they promote spliceosome activation and clarify whether it is strictly structural/conformational or if the activity of Brr2 is directly affected by the presence of these proteins.

At this moment it is not entirely clear how Smu1 and RED mediate the interaction between SF3B3 and Brr2, especially when the 5'SS-BS distance is short. One possibility is that Smu1 and RED hold SF3B3 and Brr2 close together, and that this interaction is disrupted when the intron or 5'SS-BS distance is short. Alternatively, if Smu1 and RED do not actively counteract the physical hindrance that a short 5'SS-BS distance exerts, Smu1 might simply span the gap between U2 and the tri-snRNP, when U2 is not flexible enough to move towards Brr2. In that case, subtle differences in the structure of the spliceosome might not be observable by negative stain electron microscopy. Therefore, examination of these complexes by cryo-electron microscopy might provide more detailed structural information about the conformational state of B complexes lacking Smu1 and RED, resulting in a better understanding of the function of these proteins. Likewise, cryo-EM may in the future reveal structural differences between human B complexes formed on very short versus long introns. 


\section{References}

Abebrese, E.L., Ali, S.H., Arnold, Z.R., Andrews, V.M., Armstrong, K., Burns, L., Crowder, H.R., Day, R.T., Hsu, D.G., Jarrell, K., Lee, G., Luo, Y., Mugayo, D., Raza, Z., and Friend, K. (2017). Identification of human short introns. PLOS ONE 12, 1-19.

Absmeier, E., Santos, K.F., and Wahl, M.C. (2016). Functions and regulation of the Brr2 RNA helicase during splicing. Cell Cycle 15, 3362-3377.

Achsel, T., Ahrens, K., Brahms, H., Teigelkamp, S., and Lührmann, R. (1998). The human U5-220kD protein (hPrp8) forms a stable RNA-free complex with several U5-specific proteins, including an RNA unwindase, a homologue of ribosomal elongation factor EF-2, and a novel WD-40 protein. Molecular and Cellular Biology 18, 6756-6766.

Achsel, T., Brahms, H., Kastner, B., Bachi, A., Wilm, M., and Lührmann, R. (1999). A doughnut-shaped heteromer of human Sm-like proteins binds to the 3'-end of U6 snRNA, thereby facilitating U4/U6 duplex formation in vitro. EMBO Journal 18, 5789-5802.

Agafonov, D.E., Deckert, J., Wolf, E., Odenwalder, P., Bessonov, S., Will, C.L., Urlaub, H., Luhrmann, R., Odenwälder, P., Bessonov, S., Will, C.L., Urlaub, H., and Lührmann, R. (2011). Semiquantitative proteomic analysis of the human spliceosome via a novel two-dimensional gel electrophoresis method. Molecular and Cellular Biology 31, 2667-82.

Agafonov, D.E., Kastner, B., Dybkov, O., Hofele, R. V., Liu, W.T., Urlaub, H., Lührmann, R., and Stark, H. (2016). Molecular architecture of the human U4/U6.U5 tri-snRNP. Science 351.

Agafonov, D.E., Van Santen, M., Kastner, B., Dube, P., Will, C.L., Urlaub, H., and Luhrmann, R. (2016). ATPץS stalls splicing after B complex formation but prior to spliceosome activation. Rna 22, 1329-1337.

Anokhina, M., Bessonov, S., Miao, Z., Westhof, E., Hartmuth, K., and Lührmann, R. (2013). RNA structure analysis of human spliceosomes reveals a compact $3 \mathrm{D}$ arrangement of snRNAs at the catalytic core. EMBO Journal 32, 2804-2818.

Arenas, J.E., and Abelson, J.N. (1997). Prp43: An RNA helicase-like factor involved in spliceosome disassembly. Proceedings of the National Academy of Sciences of the United States of America 94, 11798-11802.

Avery, O.T., Macleod, C.M., McCarty, M., and Peltier, L.F. (1944). Studies on the chemical nature of the substance inducing transformation of pneumococcal types: Induction of transformation by a desoxyribonucleic acid fraction isolated from Pheumococcus type III. Clinical Orthopaedics and Related Research.

Bach, M., Winkelmann, G., and Lührmann, R. (1989). 20S small nuclear ribonucleoprotein U5 shows a surprisingly complex protein composition. Proceedings of the National Academy of Sciences of the United States of America 86, 6038-42.

Bai, R., Wan, R., Yan, C., Lei, J., and Shi, Y. (2018). Structures of the fully assembled Saccharomyces cerevisiae spliceosome before activation. Science 0325, 1-11.

Bai, R., Yan, C., Wan, R., Lei, J., and Shi, Y. (2017). Structure of the Post-catalytic Spliceosome from Saccharomyces cerevisiae. Cell 171, 1589-1598.e8.

Bao, P., Will, C.L., Urlaub, H., Boon, K.L., and Lührmann, R. (2017). The RES complex is required for efficient transformation of the precatalytic B spliceosome into an activated Bactcomplex. Genes and Development 31, 2416-2429.

Bartels, C., Klatt, C., Lührmann, R., and Fabrizio, P. (2002). The ribosomal translocase homologue Snu114p is involved in unwinding U4/U6 RNA during activation of the spliceosome. EMBO Reports 3, 875-880. 


\section{References}

Baserga, S.J., and Steitz, J. a. (1993). The Diverse World of Small Ribonucleoproteins. The RNA World 359-381. Behrens, S.E., and Lührmann, R. (1991). Immunoaffinity purification of a [U4/U6.U5] tri-snRNP from human cells. Genes \& Dev. 5, 1439-1452.

Behrens, S.E., Tyc, K., Kastner, B., Reichelt, J., and Lührmann, R. (1993). Small nuclear ribonucleoprotein (RNP) U2 contains numerous additional proteins and has a bipartite RNP structure under splicing conditions. Molecular and Cellular Biology.

Behzadnia, N., Golas, M.M., Hartmuth, K., Sander, B., Kastner, B., Deckert, J., Dube, P., Will, C.L., Urlaub, H., Stark, H., and Lührmann, R. (2007). Composition and three-dimensional EM structure of double affinitypurified, human prespliceosomal A complexes. The EMBO Journal 26, 1737-48.

Bentley, D.L. (2014). Coupling mRNA processing with transcription in time and space. Nature Reviews. Genetics $15,163-75$.

Berglund, J.A., Chua, K., Abovich, N., Reed, R., and Rosbash, M. (1997). The splicing factor BBP interacts specifically with the pre-mRNA branchpoint sequence UACUAAC. Cell 89, 781-787.

Berk, A.J. (2016). Discovery of RNA splicing and genes in pieces. Proceedings of the National Academy of Sciences 113, 801-805.

Bertram, K., Agafonov, D.E., Dybkov, O., Haselbach, D., Leelaram, M.N., Will, C.L., Urlaub, H., Kastner, B., Lührmann, R., and Stark, H. (2017). Cryo-EM Structure of a Pre-catalytic Human Spliceosome Primed for Activation. Cell 170, 701-713.e11.

Bertram, K., Agafonov, D.E., Liu, W.T., Dybkov, O., Will, C.L., Hartmuth, K., Urlaub, H., Kastner, B., Stark, H., and Lührmann, R. (2017). Cryo-EM structure of a human spliceosome activated for step 2 of splicing. Nature 542, 318-323.

Bessonov, S., Anokhina, M., Krasauskas, A., Golas, M.M., Sander, B., Will, C.L., Urlaub, H., Stark, H., and Lührmann, R. (2010). Characterization of purified human Bact spliceosomal complexes reveals compositional and morphological changes during spliceosome activation and first step catalysis. RNA (New York, N.Y.) 16, 2384-403.

Bessonov, S., Anokhina, M., Will, C.L., Urlaub, H., and Lührmann, R. (2008). Isolation of an active step I spliceosome and composition of its RNP core. Nature 452, 846-50.

Beyer, A.L., and Osheim, Y.N. (1988). Splice site selection, rate of splicing, and alternative splicing on nascent transcripts. Genes \& Development 2, 754-765.

Birney, E., Kumar, S., and Krainer, a R. (1993). Analysis of the RNA-recognition motif and RS and RGG domains: conservation in metazoan pre-mRNA splicing factors. Nucleic Acids Research 21, 5803-16.

Black, D.L. (2003). Mechanisms of Alternative Pre-Messenger RNA Splicing. Annual Review of Biochemistry 72, 291-336.

Black, D.L., and Pinto, A.L. (1989). U5 small nuclear ribonucleoprotein: RNA structure analysis and ATPdependent interaction with U4/U6. Molecular and Cellular Biology 9, 3350-9.

Blencowe, B.J. (2006). Alternative splicing: new insights from global analyses. Cell 126, 37-47.

Boesler, C., Rigo, N., Agafonov, D.E., Kastner, B., Urlaub, H., Will, C.L., and Lühmann, R. (2015). Stable trisnRNP integration is accompanied by a major structural rearrangement of the spliceosome that is dependent on Prp8 interaction with the 5' splice site. Rna 21, 1993-2005.

Boesler, C., Rigo, N., Anokhina, M.M., Tauchert, M.J., Agafonov, D.E., Kastner, B., Urlaub, H., Ficner, R., Will, C.L., and Lührmann, R. (2016). A spliceosome intermediate with loosely associated tri-snRNP accumulates in the absence of Prp28 ATPase activity. Nature Communications 7.

Bonnal, S., Martínez, C., Förch, P., Bachi, A., Wilm, M., and Valcárcel, J. (2008). RBM5/Luca-15/H37 Regulates Fas Alternative Splice Site Pairing after Exon Definition. Molecular Cell 32, 81-95.

Bradley, T., Cook, M.E., and Blanchette, M. (2014). SR proteins control a complex network of RNA-processing events. RNA (New York, N.Y.) 21, 75-92. 
Bringmann, P., Appel, B., Rinke, J., Reuter, R., Theissen, H., and Lührmann, R. (1984). Evidence for the existence of snRNAs $\mathrm{U} 4$ and $\mathrm{U} 6$ in a single ribonucleoprotein complex and for their association by intermolecular base pairing. The EMBO Journal 3, 1357-63.

Brosi, R., Hauri, H.P., and Kramer, A. (1993). Separation of splicing factor SF3 into two components and purification of SF3a activity. Journal of Biological Chemistry.

Brow, D.A., and Guthrie, C. (1988). Spliceosomal RNA U6 is remarkably conserved from yeast to mammals. Nature 334, 213-218.

Caudevilla, C., Serra, D., Miliar, A., Codony, C., Asins, G., Bach, M., and Hegardt, F.G. (1998). Natural transsplicing in carnitine octanoyltransferase pre-mRNAs in rat liver. Proceedings of the National Academy of Sciences 95, 12185-12190.

Chandrasekharappa, S.C., Smith, J.H., and Eliceiri, G.L. (1983). Biosynthesis of Small Nuclear RNAs in Human Cells. Journal of Cellular Physiology 117, 169-174.

Chiu, Y.L., Ho, C.K., Saha, N., Schwer, B., Shuman, S., and Rana, T.M. (2002). Tat stimulates cotranscriptional capping of HIV mRNA. Molecular Cell 10, 585-597.

Chung, T., Wang, D., Kim, C.-S., Yadegari, R., and Larkins, B.A. (2009). Plant SMU-1 and SMU-2 Homologues Regulate Pre-mRNA Splicing and Multiple Aspects of Development. Plant Physiology 151, 1498-1512.

Clawson, G.A., Feldherr, C.M., and Smuckler, E.A. (1985). Nucleocytoplasmic RNA transport. Molecular and Cellular Biochemistry 67, 87-100.

Consortium, I.H.G.S. (2004). Finishing the euchromatic sequence of the human genome. Nature 50, 162-8.

Coolidge, C.J., Seely, R.J., and Patton, J.G. (1997). Functional analysis of the polypyrimidine tract in pre-mRNA splicing. Nucleic Acids Research 25, 888-896.

Cooper, T.A., Wan, L., and Dreyfuss, G. (2009). RNA and Disease. Cell 136, 777-793.

Cordin, O., and Beggs, J.D. (2013). RNA helicases in splicing. RNA Biology 6286, 83-95.

Das, R., Zhou, Z., and Reed, R. (2000). Functional association of U2 snRNP with the ATP-independent splicecosomal complex E. Molecular Cell 5, 779-787.

David, C.J., Boyne, A.R., Millhouse, S.R., and Manley, J.L. (2011). The RNA polymerase II C-terminal domain promotes splicing activation through recruitment of a U2AF65-Prp19 complex. Genes and Development 25, 972-982.

de la Mata, M., Alonso, C.R., Fededa, J.P., Pelisch, F., Cramer, P., Bentley, D., and Kornblihtt, A.R. (2003). A Slow RNA Polymerase II Affects Alternative Splicing In Vivo. Malecular Cell 12, 525-532.

de la Mata, M., and Kornblihtt, A.R. (2006). RNA polymerase II C-terminal domain mediates regulation of alternative splicing by SRp20. Nature Structural \& Molecular Biology 13, 973-980.

Deckert, J., Hartmuth, K., Boehringer, D., Behzadnia, N., Will, C.L., Kastner, B., Stark, H., Urlaub, H., and Lührmann, R. (2006). Protein composition and electron microscopy structure of affinity-purified human spliceosomal B complexes isolated under physiological conditions. Molecular and Cellular Biology 26, 552843.

Dignamr, J.D., Lebovitz, R.M., and Roeder, R.G. (1983). Accurate transcription initiation by RNA polymerase II in a soluble extract from isolated mammalian nuclei. Nucleic Acids Research 36, 1475-1489.

Dujardin, G., Lafaille, C., de la Mata, M., Marasco, L.E., Muñoz, M.J., Le Jossic-Corcos, C., Corcos, L., and Kornblihtt, A.R. (2014). How Slow RNA Polymerase II Elongation Favors Alternative Exon Skipping. Molecular Cell 54, 683-690.

Fabrizio, P., Dannenberg, J., Dube, P., Kastner, B., Stark, H., Urlaub, H., and Lührmann, R. (2009). The Evolutionarily Conserved Core Design of the Catalytic Activation Step of the Yeast Spliceosome. Molecular Cell 36, 593-608.

Fica, S.M., Oubridge, C., Galej, W.P., Wilkinson, M.E., Bai, X.C., Newman, A.J., and Nagai, K. (2017). Structure of a spliceosome remodelled for exon ligation. Nature 542, 377-380. 


\section{References}

Fica, S.M., Tuttle, N., Novak, T., Li, N.-S., Lu, J., Koodathingal, P., Dai, Q., Staley, J.P., and Piccirilli, J.A. (2013). RNA catalyzes nuclear pre-mRNA splicing. Nature 25, 368-379.

Fischer, U., Liu, Q., and Dreyfuss, G. (1997). The SMN-SIP1 complex has an essential role in spliceosomal snRNP biogenesis. Cell 90, 1023-1029.

Fischer, U., and Lührmann, R. (1990). An essential signaling role for the m3G cap in the transport of U1 snRNP to the nucleus. Science 249, 786-790.

Fong, Y.W., and Zhou, Q. (2001). Stimulatory effect of splicing factors on transcriptional elongation. Nature 414, 929-933.

Fournier, G., Chiang, C., Munier, S., Tomoiu, A., Demeret, C., Vidalain, P.O., Jacob, Y., and Naffakh, N. (2014). Recruitment of RED-SMU1 Complex by Influenza A Virus RNA Polymerase to Control Ciral mRNA Splicing. PLoS Pathogens 10.

Fu, X.Y., Colgan, J.D., and Manley, J.L. (1988). Multiple cis-acting sequence elements are required for efficient splicing of simian virus 40 small-t antigen pre-mRNA. Molecular and Cellular Biology 8, 3582-3590.

Furuichi, Y., LaFiandra, A., and Shaktin, A.J. (1977). 5'-Terminal structure and mRNA stability. Nature 269, 235239.

Galej, W.P., Wilkinson, M.E., Fica, S.M., Oubridge, C., Newman, A.J., and Nagai, K. (2016). Cryo-EM structure of the spliceosome immediately after branching. Nature 537, 197-201.

Garcia-Blanco, M.A. (2003). Messenger RNA reprogramming by spliceosome-mediated RNA trans-splicing. Journal of Clinical Investigation 112, 474-480.

Gee, S., Krauss, S.W., Miller, E., Aoyagi, K., Arenas, J., and Conboy, J.G. (1997). Cloning of mDEAH9, a putative RNA helicase and mammalian homologue of Saccharomyces cerevisiae splicing factor Prp43. Proceedings of the National Academy of Sciences of the United States of America 94, 11803-11807.

Geuens, T., Bouhy, D., and Timmerman, V. (2016). The hnRNP family: insights into their role in health and disease. Human Genetics 135, 851-867.

Girard, C., Will, C.L., Peng, J., Makarov, E.M., Kastner, B., Lemm, I., Urlaub, H., Hartmuth, K., Luhrmann, R., and Lührmann, R. (2012). Post-transcriptional spliceosomes are retained in nuclear speckles until splicing completion. Nature Communications 3, 994.

Goldstrohm, A.C., Greenleaf, A.L., and Garcia-Blanco, M.A. (2001). Co-transcriptional splicing of premessenger RNAs: Considerations for the mechanism of alternative splicing. Gene 277, 31-47.

Gozani, O., Feld, R., and Reed, R. (1996). Evidence that sequence-independent binding of highly conserved U2 snRNP proteins upstream of the branch site is required for assembly of spliceosomal complex A. Genes and Development 10, 233-243.

Gozani, O., Potashkin, J., and Reed, R. (1998). A Potential Role for U2AF-SAP 155 Interactions in Recruiting U2 snRNP to the Branch Site. Molecular and Cellular Biology 18, 4752-4760.

Hamm, J., and Mattaj, I.W. (1990). Monomethylated cap structures facilitate RNA export from the nucleus. Cell 63, 109-118.

Han, S.P., Tang, Y.H., and Smith, R. (2010). Functional diversity of the hnRNPs: past, present and perspectives. Biochemical Journal 430, 379-392.

Hang, J., Wan, R., Yan, C., and Shi, Y. (2015). Structural basis of pre-mRNA splicing. Science 349, 1191-1198.

Haselbach, D., Komarov, I., Agafonov, D.E., Hartmuth, K., Graf, B., Dybkov, O., Urlaub, H., Kastner, B., Lührmann, R., and Stark, H. (2018). Structure and Conformational Dynamics of the Human Spliceosomal BactComplex. Cell 172, 454-464.e11.

Hashimoto, C., and Steitz, J.A. (1984). U4 and U6 RNAs coexist in a single small nuclear ribonucleoprotein particle. Nucleic Acids Research 12, 3283-3293. 
Hegele, A., Kamburov, A., Grossmann, A., Sourlis, C., Wowro, S., Weimann, M., Will, C.L., Pena, V., Lührmann, R., and StelzI, U. (2012). Dynamic Protein-Protein Interaction Wiring of the Human Spliceosome. Molecular Cell 45, 567-580.

Heinrichs, V., Bach, M., Winkelmann, G., and Luhrmann, R. (1990). U1-specific C protein needed for efficient complex formation of U1 snRNP with a 5' splice site. Science 247, 69-72.

Henning, L.M., Santos, K.F., Sticht, J., Jehle, S., Lee, C.T., Wittwer, M., Urlaub, H., Stelzl, U., Wahl, M.C., and Freund, C. (2017). A new role for FBP21 as regulator of Brr2 helicase activity. Nucleic Acids Research 45, 7922-7937.

Hernández, H., Makarova, O. V., Makarov, E.M., Morgner, N., Muto, Y., Krummel, D.P., and Robinson, C. V. (2009). Isoforms of U1-70k control subunit dynamics in the human spliceosomal U1 snRNP. PLoS ONE 4.

Himmelspach, M., Gattoni, R., Gerst, C., Chebli, K., and Stévenin, J. (1991). Differential block of U small nuclear ribonucleoprotein particle interactions during in vitro splicing of adenovirus E1A transcripts containing abnormally short introns. Molecular and Cellular Biology 11, 1258-1269.

Hirose, Y., Tacke, R., and Manley, J.L. (1999). Phosphorylated RNA polymerase II stimulates pre-mRNA splicing. Genes \& Development.

House, A.E., and Lynch, K.W. (2006). An exonic splicing silencer represses spliceosome assembly after ATPdependent exon recognition. Nature Structural and Molecular Biology 13, 937-944.

Izquierdo, J.M., Majós, N., Bonnal, S., Martínez, C., Castelo, R., Guigó, R., Bilbao, D., and Valcárcel, J. (2005). Regulation of fas alternative splicing by antagonistic effects of TIA-1 and PTB on exon definition. Molecular Cell 19, 475-484.

Jacob, F., and Monod, J. (1961). Genetic regulatory mechanisms in the synthesis of proteins. Journal of Molecular Biology 3, 318-356.

Keating, K.S., Toor, N., Perlman, P.S., and Pyle, A.M. (2010). A structural analysis of the group II intron active site and implications for the spliceosome. RNA (New York, N.Y.) 1-9.

Kent, O.A., and MacMillan, A.M. (2002). Early organization of pre-mRNA during spliceosome assembly. Nature Structural Biology 9, 576-581.

Köhrer, K., and Domdey, H. (1988). Splicing and spliceosome formation of the yeast MATal transcript require a miniimum distance from the 5' splice site to the internal branch acceptor site. Nucleic Acids Research 16, 9457-9475.

Konarska, M.M., Padgett, R.A., and Sharp, P.A. (1985). Trans-splicing of mRNA precursors in vitro . Cell 42, 165-171.

Krainer, A.R., Maniatis, T., Ruskin, B., and Green, M.R. (1984). Normal and mutant human $\beta$-globin pre-mRNAs are faithfully and efficiently spliced in vitro. Cell 36, 993-1005.

Kunkel, G.R., Maser, R.L., Calvet, J.P., and Pederson, T. (1986). U6 small nuclear RNA is transcribed by RNA polymerase III. Proceedings of the National Academy of Sciences of the United States of America 83, 85758579.

Laemmli, U.K. (1970). Cleavage of Structural Proteins during the Assembly of the Head of Bacteriophage T4. Nature 227, 680 .

Laggerbauer, B., Achsel, T., and Lührmann, R. (1998). The human U5-200kD DEXH-box protein unwinds U4/U6 RNA duplices in vitro. Proceedings of the National Academy of Sciences of the United States of America 95, 4188-4192.

Lam, B.J., and Hertel, K.J. (2002). A general role for splicing enhancers in exon definition. RNA (New York, N.Y.) 8, 1233-1241.

Lambowitz, A.M., and Zimmerly, S. (2011). Group II introns: Mobile ribozymes that invade DNA. Cold Spring Harbor Perspectives in Biology 3, 1-19. 


\section{References}

Lamond, A.I., Konarska, M.M., and Sharp, P.A. (1987). A mutational analysis of spliceosome assembly: evidence for splice site collaboration during spliceosome formation. Genes \& Development 1, 532-543.

Lander, E.S., Linton, L.M., and Birren, B. (2001). Initial sequencing and analysis of the human genome. Nature 409, 860-921.

Lardelli, R.M., Thompson, J.X., Yates, J.R., and Stevens, S.W. (2010). Release of SF3 from the intron branchpoint activates the first step of pre-mRNA splicing. RNA (New York, N.Y.).

Le Hir, H., Izaurralde, E., Maquat, L.E., and Moore, M.J. (2000). The spliceosome deposits multiple proteins 2024 nucleotides upstream of mRNA exon-exon junctions. EMBO Journal 19, 6860-6869.

Le Hir, H., and Séraphin, B. (2008). EJCs at the Heart of Translational Control. Cell 133, 213-216.

Lerner, M.R., and Steitz, J.A. (1979). Antibodies to small nuclear RNAs complexed with proteins are produced by patients with systemic lupus erythematosus. Proceedings of the National Academy of Sciences of the United States of America 76, 5495-5499.

Liang, F., Holt, I., Pertea, G., Karamycheva, S., Salzberg, S.L., and Quackenbush, J. (2000). Gene index analysis of the human genome estimates approximately 120,000 genes. Nature Genetics 25, 239-240.

Lim, S.R., and Hertel, K.J. (2004). Commitment to splice site pairing coincides with a complex formation. Molecular Cell 15, 477-483.

Liu, S., Li, X., Zhang, L., Jiang, J., Hill, R.C., Cui, Y., Hansen, K.C., Zhou, Z.H., and Zhao, R. (2017). Structure of the yeast spliceosomal postcatalytic $\mathrm{P}$ complex. Science 358, 1278-1283.

Lopez, P.J., and Séraphin, B. (1999). Genomic-scale quantitative analysis of yeast pre-mRNA splicing: implications for splice-site recognition. RNA (New York, N.Y.) 5, 1135-1137.

Lybarger, S., Beickman, K., Brown, V., Dembla-Rajpal, N., Morey, K., Seipelt, R., and Rymond, B.C. (1999). Elevated levels of a U4/U6.U5 snRNP-associated protein, Spp381p, rescue a mutant defective in spliceosome maturation. Molecular and Cellular Biology 19, 577-584.

MacMillan, A.M., Query, C.C., Allerson, C.R., Chen, S., Verdine, G.L., and Sharp, P.A. (1994). Dynamic association of proteins with the pre-mRNA branch region. Genes and Development 8, 3008-3020.

Madhani, H.D., and Guthrie, C. (1992). A novel base-pairing interaction between U2 and U6 snRNAs suggests a mechanism for the catalytic activation of the spliceosome. Cell 71, 803-817.

Maeder, C., Kutach, A.K., and Guthrie, C. (2008). ATP-dependent unwinding of U4/U6 snRNAs by the Brr2 helicase requires the C terminus of Prp8. Nature Structural and Molecular Biology 16, 42-48.

Makarov, E.M., Makarova, O. V., Achsel, T., and Lührmann, R. (2000). The human homologue of the yeast splicing factor Prp6p contains multiple TPR elements and is stably associated with the U5 snRNP via proteinprotein interactions. Journal of Molecular Biology 298, 567-575.

Makarov, E.M., Makarova, O. V., Urlaub, H., Gentzel, M., Will, C.L., Wilm, M., and Lührmann, R. (2002). Small nuclear ribonucleoprotein remodeling during catalytic activation of the spliceosome. Science 298, 2205-2208.

Makarova, O. V., Makarov, E.M., Liu, S., Vornlocher, H.P., and Lührmann, R. (2002). Protein 61K, encoded by a gene (PRPF31) linked to autosomal dominant retinitis pigmentosa, is required for U4/U6. U5 tri-snRNP formation and pre-mRNA splicing. EMBO Journal 21, 1148-1157.

Makarova, O. V., Makarov, E.M., and Lührmann, R. (2001). The 65 and 110 kDA SR-related proteins of the U4/U6. U5 tri-snRNP are essential for the assembly of mature spliceosomes. EMBO Journal 20, 2553-2563.

Makarova, O. V., Makarov, E.M., Urlaub, H., Will, C.L., Gentzel, M., Wilm, M., and Lührmann, R. (2004). A subset of human 35S U5 proteins, including Prp19, function prior to catalytic step 1 of splicing. EMBO Journal 23, 2381-2391.

Mattaj, I.W. (1986). Cap trimethylation of $U$ snRNA is cytoplasmic and dependent on $U$ snRNP protein binding. Cell $46,905-911$.

Mayas, R.M., Maita, H., and Staley, J.P. (2006). Exon ligation is proofread by the DExD/H-box ATPase Prp22p. Nature Structural and Molecular Biology 13, 482-490. 
Merendino, L., Guth, S., Bilbao, D., Concepcion, M., and Juan, V. (1999). Inhibition of msl-2 splicing by Sexlethal reveals interaction between U2AF35 and the 3' splice site AG. Nature 402, 838-841.

Millhouse, S., and Manley, J.L. (2005). The C-Terminal Domain of RNA Polymerase II Functions as a Phosphorylation-Dependent Splicing Activator in a Heterologous Protein. Molecular and Cellular Biology 25, 533-544.

Misteli, T., and Spector, D.L. (1999). RNA polymerase II targets pre-mRNA splicing factors to transcription sites in vivo. Molecular Cell 3, 697-705.

Moore, M.J., and Sharp, P.A. (1993). Evidence for 2 active sites in the spliceosome provided by stereochemistry of pre-messenger RNA splicing. Nature 365, 364-368.

Morris, D.P., and Greenleaf, A.L. (2000). The splicing factor, Prp40, binds the phosphorylated carboxyl-terminal domain of RNA Polymerase II. Journal of Biological Chemistry 275, 39935-39943.

Mozaffari-Jovin, S., Santos, K.F., Hsiao, H.H., Will, C.L., Urlaub, H., Wahl, M.C., and Lührmann, R. (2012). The Prp8 RNase H-like domain inhibits Brr2-mediated U4/U6 snRNA unwinding by blocking Brr2 loading onto the U4 snRNA. Genes and Development 26, 2422-2434.

Nagaike, T., Logan, C., Hotta, I., Rozenblatt-Rosen, O., Meyerson, M., and Manley, J.L. (2011). Transcriptional Activators Enhance Polyadenylation of mRNA Precursors. Molecular Cell 41, 409-418.

Nesic, D., Tabackovic, G., and Krämer, A. (2004). A role for Cajal bodies in the final steps of U2 snRNP biogenesis. Journal of Cell Science 117, 4423-4433.

Neugebauer, K.M. (2002). On the importance of being co-transcriptional. Journal of Cell Science 115, 3865-3871.

Newman, A. (2008). RNA Interactions in mRNA Splicing. Encyclopedia of Life Sciences 1-7.

Nguyen, T.H.D., Galej, W.P., Bai, X., Oubridge, C., Newman, A.J., Scheres, S.H.W., and Nagai, K. (2016). CryoEM structure of the yeast U4 / U6 . U5 tri-snRNP at 3 . $7 \AA$ resolution. Nature 530, 298-302.

Nguyen, T.H.D., Galej, W.P., Bai, X.C., Savva, C.G., Newman, A.J., Scheres, S.H.W., and Nagai, K. (2015). The architecture of the spliceosomal U4/U6.U5 tri-snRNP. Nature 523, 47-52.

Ohrt, T., Odenwälder, P., Dannenberg, J., Prior, M., Warkocki, Z., Schmitzová, J., Karaduman, R., Gregor, I., Enderlein, J., Fabrizio, P., and Lührmann, R. (2013). Molecular dissection of step 2 catalysis of yeast premRNA splicing investigated in a purified system. RNA (New York, N.Y.) 19, 1-14.

Ohrt, T., Prior, M., Dannenberg, J., Odenwälder, P., Dybkov, O., Rasche, N., Schmitzová, J., Gregor, I., Fabrizio, P., Enderlein, J., and Lührmann, R. (2012). Prp2-mediated protein rearrangements at the catalytic core of the spliceosome as revealed by dcFCCS. RNA (New York, N.Y.) 18, 1244-1256.

Pan, Q., Shai, O., Lee, L.J., Frey, B.J., and Blencowe, B.J. (2008). Deep surveying of alternative splicing complexity in the human transcriptome by high-throughput sequencing. Nature Genetics 40, 1413-1415.

Pandya-Jones, A.M.Y., and Black, D.L. (2009). Co-transcriptional splicing of constitutive and alternative exons. RNA (New York, N.Y.) 15, 1896-1908.

Papasaikas, P., Tejedor, J.R., Vigevani, L., and Valcárcel, J. (2015). Functional splicing network reveals extensive regulatory potential of the core spliceosomal machinery. Molecular Cell 57, 7-22.

Plaschka, C., Lin, P.C., and Nagai, K. (2017). Structure of a pre-catalytic spliceosome. Nature 546, 617-621.

Query, C.C., Moore, M.J., and Sharp, P.A. (1994). Branch nucleophile selection in pre-mRNA splicing: Evidence for the bulged duplex model. Genes and Development 8, 587-597.

Raghunathan, P.L., and Guthrie, C. (1998). RNA unwinding in U4/U6 snRNPs requires ATP hydrolysis and the DEIH-box splicing factor Brr2. Current Biology 8, 847-855.

Raker, V.A., Plessel, G., and Lührmann, R. (1996). The snRNP core assembly pathway: identification of stable core protein heteromeric complexes and an snRNP subcore particle in vitro. The EMBO Journal 15, 225669.

Ram, O., and Ast, G. (2006). SR proteins: a foot on the exon before the transition from intron to exon definition. Trends in Genetics 23, 5-7. 


\section{References}

Rauhut, R., Fabrizio, P., Dybkov, O., Hartmuth, K., Pena, V., Chari, A., Kumar, V., Lee, C., and Urlaub, H. (2016). Molecular architechture of the Saccharomyces cerevisiae activated spliceosome. Science (New York, N.Y.) 353, 1399-1405.

Reyes, J.L., Kois, P., Konforti, B.B., and Konarska, M.M. (1996). The canonical GU dinucleotide at the 5' splice site is recognized by p220 of the U5 snRNP within the spliceosome. RNA (New York, N.Y.).

Robberson, B.L., Cote, G.J., and Berget, S.M. (1990). Exon definition may facilitate splice site selection in RNAs with multiple exons. Molecular and Cellular Biology 10, 84-94.

Rosonina, E., Bakowski, M.A., McCracken, S., and Blencowe, B.J. (2003). Transcriptional Activators Control Splicing and 3'-End Cleavage Levels. Journal of Biological Chemistry 278, 43034-43040.

Ruskin, B., Greene, J.M., and Green, M.R. (1985). Cryptic branch point activation allows accurate in vitro splicing of human $\beta$-globin intron mutants. Cell 41, 833-844.

Ruskin, B., Zamore, P.D., and Green, M.R. (1988). A factor, U2AF, is required for U2 snRNP binding and splicing complex assembly. Cell 52, 207-219.

Sakharkar, M.K., Chow, V.T.K., and Kangueane, P. (2004). Distributions of exons and introns in the human genome. In Silico Biology 4, 387-93.

Sambrook, J., Fritsch, E.F., and Maniatis, T. (1989). Molecular cloning: a laboratory manual, 2nd ed. Cold Spring Harbor Laboratory, Cold Spring Harbor, N.Y.

Schaffert, N., Hossbach, M., Heintzmann, R., Achsel, T., and L??hrmann, R. (2004). RNAi knockdown of hPrp31 to an accumulation of U4/U6 di-snRNPs in Cajal bodies. EMBO Journal 23, 3000-3009.

Schellenberg, M.J., Edwards, R.A., Ritchie, D.B., Kent, O.A., Golas, M.M., Stark, H., Luhrmann, R., Glover, J.N.M., and MacMillan, A.M. (2006). Crystal structure of a core spliceosomal protein interface. Proceedings of the National Academy of Sciences 103, 1266-1271.

Schneider, M., Hsiao, H.H., Will, C.L., Giet, R., Urlaub, H., and Lührmann, R. (2010). Human PRP4 kinase is required for stable tri-snRNP association during spliceosomal B complex formation. Nature Structural and Molecular Biology 17, 216-221.

Schneider, M., Will, C.L., Anokhina, M., Tazi, J., Urlaub, H., and Lührmann, R. (2010). Exon Definition Complexes Contain the Tri-snRNP and Can Be Directly Converted into B-like Precatalytic Splicing Complexes. Molecular Cell 38, 223-235.

Schütze, T., Ulrich, A.K.C., Apelt, L., Will, C.L., Bartlick, N., Seeger, M., Weber, G., Lührmann, R., Stelzl, U., and Wahl, M.C. (2016). Multiple protein-protein interactions converging on the Prp38 protein during activation of the human spliceosome. RNA (New York, N.Y.) 22, 265-277.

Schwer, B. (2001). A new twist on RNA helicases : DExH / D box proteins as RNPases. Nature 8, 113-116.

Schwer, B. (2008). A Conformational Rearrangement in the Spliceosome Sets the Stage for Prp22-Dependent mRNA Release. Molecular Cell 30, 743-754.

Schwer, B., and Guthrie, C. (1991). PRP16 is an RNA-dependent ATPase that interacts transiently with the spliceosome. Nature 349, 494-499.

Scotti, M.M., and Swanson, M.S. (2016). RNA mis-splicing in disease. Nature Reviews Genetics 17, 19-32.

Seetharaman, M., Eldho, N. V., Padgett, R.A., and Dayie, K.T. (2006). Structure of a self-splicing group II intron catalytic effector domain 5: Parallels with spliceosomal U6 RNA. RNA (New York, N.Y.) 12, 235-247.

Semlow, D.R., and Staley, J.P. (2012). Staying on message: ensuring fidelity in pre-mRNA splicing. Trends Biochem Sciences 37, 263-273.

Sharma, S., Kohlstaedt, L.A., Damianov, A., Rio, D.C., and Black, D.L. (2008). Polypyrimidine tract binding protein controls the transition from exon definition to an intron defined spliceosome. Nature Structural and Molecular Biology 15, 183-191.

Shatkin, A.J. (1976). Capping of eucaryotic mRNAs. Cell 9, 645-653. 
Shatkin, A.J., and Manley, J.L. (2000). The ends of the affair: Capping and polyadenylation. Nature Structural Biology 7, 838-842.

Shepard, P.J., and Hertel, K.J. (2009). The SR protein family. Genome Biology 10, 242.

Shevchenko, A., Wilm, M., Vorm, O., and Mann, M. (1996). Mass spectrometric sequencing of proteins silverstained polyacrylamide gels. Analytical Chemistry 68, 850-858.

Shi, Y., Reddy, B., and Manley, J.L. (2006). PP1/PP2A phosphatases are required for the second step of PremRNA splicing and target specific snRNP proteins. Molecular Cell 23, 819-29.

Shumyatsky, G., Wright, D., and Reddy, R. (1993). Methylphosphate cap structure increases the stability of 7SK, B2 and U6 small RNAs in Xenopus oocytes. Nucleic Acids Research 21, 4756-4761.

Sidarovich, A., Will, C.L., Anokhina, M.M., Ceballos, J., Sievers, S., Agafonov, D.E., Samatov, T., Bao, P., Kastner, B., Urlaub, H., Waldmann, H., and Lührmann, R. (2017). Identification of a small molecule inhibitor that stalls splicing at an early step of spliceosome activation. ELife 6, 1-32.

Sloan, K.E., and Bohnsack, M.T. (2018). Unravelling the Mechanisms of RNA Helicase Regulation. Trends in Biochemical Sciences 43, 237-250.

Small, E.C., Leggett, S.R., Winans, A.A., and Staley, J.P. (2006). The EF-G-like GTPase Snu114p Regulates Spliceosome Dynamics Mediated by Brr2p, a DExD/H Box ATPase. Molecular Cell 23, 389-399.

Smith, C.W.J., and Nadal-Ginard, B. (1989). Mutually exclusive splicing of $\alpha$-tropomyosin exons enforced by an unusual lariat branch point location: Implications for constitutive splicing. Cell 56, 749-758.

Sontheimer, E.J., and Steitz, J.A. (1993). The U5 and U6 small nuclear RNAs as active site components of the spliceosome. Science 262, 1989-1996.

Spartz, A.K., Herman, R.K., and Shaw, J.E. (2004). SMU-2 and SMU-1, Caenorhabditis elegans Homologs of Mammalian Spliceosome-Associated Proteins RED and fSAP57, Work Together To Affect Splice Site Choice. Molecular and Cellular Biology 24, 6811-6823.

Spiller, M.P., Boon, K.L., Reijns, M.A.M., and Beggs, J.D. (2007). The Lsm2-8 complex determines nuclear localization of the spliceosomal U6 snRNA. Nucleic Acids Research 35, 923-929.

Spingola, M., Grate, L., Haussler, D., and Manuel, A. (1999). Genome-wide bioinformatic and molecular analysis of introns in Saccharomyces cerevisiae. RNA (New York, N.Y.) 5, 221-234.

Staley, J.P., and Guthrie, C. (1998). Mechanical devices of the spliceosome: Motors, clocks, springs, and things. Cell 92, 315-326.

Staley, J.P., and Guthrie, C. (1999). An RNA switch at the 5' splice site requires ATP and the DEAD box protein Prp28p. Molecular Cell 3, 55-64.

Sterner, D.A., Carlo, T., and Berget, S.M. (1996). Architectural limits on split genes. Proceedings of the National Academy of Sciences of the United States of America 93, 15081-15085.

Sun, J.S., and Manley, J.L. (1995). A novel U2-U6 snRNA structure is necessary for mammalian mRNA splicing. Genes and Development 9, 843-854.

Teigelkamp, S., Newman, a J., and Beggs, J.D. (1995). Extensive interactions of PRP8 protein with the 5' and 3 ' splice sites during splicing suggest a role in stabilization of exon alignment by U5 snRNA. EMBO Journal 14, 2602-2612.

Ulfendahl, P.J., Pettersson, U., and Akusjarvi, G. (1985). Splicing of the adenovirus-2 EIA 13S mRNA requires a minimal intron length and specific intron signals. Nucleic Acids Research 36, 6299-6315.

Ulrich, A.K.C., Schulz, J.F.F., Kamprad, A., Schütze, T., and Wahl, M.C.C. (2016). Structural Basis for the Functional Coupling of the Alternative Splicing Factors Smu1 and RED. Structure 24, 762-773.

Ulrich, A.K.C., and Wahl, M.C. (2017). Human MFAP1 is a cryptic ortholog of the Saccharomyces cerevisiae Spp381 splicing factor. BMC Evolutionary Biology 17.

Umen, J.G., and Guthrie, C. (1995). A Novel Role for a U5 snRNP Protein in 3'-Splice-Site Selection. Genes \& Development 9, 855-868. 


\section{References}

Wahl, M.C., Will, C.L., and Lührmann, R. (2009). The spliceosome: design principles of a dynamic RNP machine. Cell 136, 701-718.

Wan, R., Yan, C., Bai, R., Huang, G., and Shi, Y. (2016). Structure ofa yeast catalytic step I spliceosome at $3.4 \AA$ resolution. Science 353, 895-904.

Wan, R., Yan, C., Bai, R., Lei, J., and Shi, Y. (2017). Structure of an Intron Lariat Spliceosome from Saccharomyces cerevisiae. Cell 171, 120-132.e12.

Wang, E.T., Sandberg, R., Luo, S., Khrebtukova, I., Zhang, L., Mayr, C., Kingsmore, S.F., Schroth, G.P., and Burge, C.B. (2008). Alternative isoform regulation in human tissue transcriptomes. Nature 456, 470-476.

Wang, Y., Liu, J., Huang, B., Xu, Y., Li, J., Huang, L., Lin, J., Zhang, J., Min, Q., Yang, W., and Wang, X. (2015). Mechanism of alternative splicing and its regulation (Review). Biomedical Reports 3, 152-158.

Watson, J., and Crick, F. (1953). Molecular structure of nucleic acids. Nature. 171, 737-738.

Webb, T.R., Joyner, A.S., and Potter, P.M. (2013). The development and application of small molecule modulators of SF3b as therapeutic agents for cancer. Drug Discovery Today 18, 43-49.

Wersig, C., Guddat, U., Pieler, T., and Bindereif, A. (1992). Assembly and nuclear transport of the U4 and U4 U6 snRNPs. Experimental Cell Research 199, 373-377.

Wieringa, B., Hofer, E., and Weissmann, C. (1984). A minimal intron length but no specific internal sequence is required for splicing the large rabbit $\beta$-globin intron. Cell 37, 915-925.

Wilkinson, M.E., Fica, S.M., Galej, W.P., Norman, C.M., Newman, A.J., and Nagai, K. (2017). Postcatalytic spliceosome structure reveals mechanism of 3 '-splice site selection. Science 358, 1283-1287.

Will, C.L., and Lührmann, R. (2011). Spliceosome structure and function. Cold Spring Harbor Perspectives in Biology 3, 1-23.

Will, C.L., Schneider, C., Macmillan, A.M., Katopodis, N.F., Neubauer, G., Wilm, M., Lührmann, R., and Query, C.C. (2001). A novel U2 and U11/U12 snRNP protein that associates with the pre-mRNA branch site. EMBO Journal 20, 4536-4546.

Will, C.L., Urlaub, H., Achsel, T., Gentzel, M., Wilm, M., and Lührmann, R. (2002). Characterization of novel SF3b and 17S U2 snRNP proteins, including a human Prp5p homologue and an SF3b DEAD-box protein. EMBO Journal 21, 4978-4988.

Woppmann, A., Will, C.L., Kornstädt, U., Zuo, P., Manley, J.L., and Lührmann, R. (1993). Identification of an snrnp-associated kinase activity that phosphorylates arginine/serine rich domains typical of splicing factors. Nucleic Acids Research 21, 2815-2822.

Wu, J., and Manley, J.L. (1989). Mammalian pre-mRNA branch site selection by U2 snRNP involves base pairing. Genes \& Development 3, 1553-1561.

Wu, J.Y., and Maniatis, T. (1993). Specific interactions between proteins implicated in splice site selection and regulated alternative splicing. Cell 75, 1061-1070.

Xiao, S.H., and Manley, J.L. (1997). Phosphorylation of the ASF/SF2 RS domain affects both protein-protein and protein-RNA interactions and is necessary for splicing. Genes \& Development 11, 334-344.

Xie, J., Beickman, K., Otte, E., and Rymond, B.C. (1998). Progression through the spliceosome cycle requires Prp38p function for U4/U6 snRNA dissociation. The EMBO Journal 17, 2938-2946.

Yan, C., Wan, R., Bai, R., Huang, G., and Shi, Y. (2016). Structure ofa yeast activated spliceosome at $3.5 \AA$ resolution. Science 353, 904-911.

Yan, C., Wan, R., Bai, R., Huang, G., and Shi, Y. (2017). Structure of a yeast step II catalytically activated spliceosome. Science 355, 149-155.

Yura, K., Shionyu, M., Hagino, K., Hijikata, A., Hirashima, Y., Nakahara, T., Eguchi, T., Shinoda, K., Yamaguchi, A., Takahashi, K.-I., Itoh, T., Imanishi, T., Gojobori, T., and Go, M. (2006). Alternative splicing in human transcriptome: functional and structural influence on proteins. Gene 380, 63-71.

Zhan, X., Yan, C., Zhang, X., Lei, J., and Shi, Y. (2018). Structure of a human catalytic step I spliceosome. Science 
359, 537-545.

Zhang, D., and Rosbash, M. (1999). Identification of eight proteins that cross-link to pre-mRNA in the yeast commitment complex. Genes and Development 13, 581-592.

Zhang, M., and Green, M.R. (2001). Identification and characterization of yUAP/Sub2p, a yeast homolog of the essential human pre-mRNA splicing factor hUAP56. Genes and Development 15, 30-35.

Zhang, M.Q. (1998). Statistical features of human exons and their flanking regions. Human Molecular Genetics 7 , 919-932.

Zhang, X., Yan, C., Zhan, X., Li, L., Lei, J., and Shi, Y. (2018). Structure of the human activated spliceosome in three conformational states. Cell Research 28, 307-322.

Zhuang, Y., and Weiner, A.M. (1986). Compensatory Base Change in UI snRNA suppresses a 5 ' Splice Site Mutation. Cell 46, 827-835.

Zhuang, Y., and Weiner, A.M. (1989). A compensatory base change in human U2 snRNP can supress a branch site mutation. Genes and Development 3, 1545-1552.

Zillmann, M., Zapp, M.L., and Berget, S.M. (1988). Gel electrophoretic isolation of splicing complexes containing U1 small nuclear ribonucleoprotein particles. Molecular and Cellular Biology 8, 814-21. 


\section{Appendix}

\section{Appendix}

\subsection{Abbreviations}

\begin{tabular}{|c|c|}
\hline 2'O-me & 2'O-ribose methylated \\
\hline 3'SS & 3' splice site \\
\hline 5 'SS & 5' splice site \\
\hline$\Delta$ & Depletion / mutant \\
\hline$\AA$ & Ångström unit \\
\hline$A$ & Adenosine / ampere \\
\hline aa & Amino acid \\
\hline Alt3'ss & Alternative 3'SS \\
\hline Alt5'ss & Alternative 5'SS \\
\hline Amp & Ampicillin \\
\hline APS & Ammonium peroxodisulfate \\
\hline ATP & Adenosine triphosphate \\
\hline B1-3 & Bridge 1-3 \\
\hline BCA & Bicinchoninic acid \\
\hline bp & Base pair \\
\hline $\mathrm{BP}$ & Branch point \\
\hline BPS & Branch point sequence \\
\hline BS & Branch site \\
\hline BSA & Bovine serum albumin \\
\hline${ }^{\circ} \mathrm{C}$ & Degree celsius \\
\hline C & Cytosine / Carboxy \\
\hline C. elegans & Caenorhabditis elegans \\
\hline Cex & Cassette exon \\
\hline $\mathrm{Ci}$ & Curie \\
\hline $\mathrm{cm}$ & Centimeter \\
\hline cpm & Counts per minute \\
\hline CTD & C-terminal domain \\
\hline CTP & Cytosine triphosphate \\
\hline CTRL & Control \\
\hline d & desoxy \\
\hline D5 & Domain $\mathrm{V}$ of group II introns \\
\hline dd & Double distilled/ didesoxy \\
\hline $\mathrm{Da}$ & Dalton \\
\hline
\end{tabular}




\begin{tabular}{|c|c|}
\hline $\mathrm{DExD} / \mathrm{H}$ & Consensus sequence of helicases \\
\hline DMP & dimethyl pimelimidate \\
\hline DMSO & Dimethyl sulfoxide \\
\hline DNA & Desoxyribonucleic acid \\
\hline DSP & dithiobis(succinimidyl propionate) \\
\hline DTT & Dithiothreitol \\
\hline$E C L$ & Enhanced chemiluminescence \\
\hline E. coli & Escherichia coli \\
\hline EDTA & Ethylendiamintetraacetate \\
\hline EJC & Exon junction complex \\
\hline EM & Electron microscopy \\
\hline ESE & Exonic splicing enhancer \\
\hline ESS & Exonic splicing silencer \\
\hline et al. & Et alii \\
\hline$f$ & Femto \\
\hline FBS & Fetal bovine serum \\
\hline $\mathrm{FL}$ & Full length \\
\hline Fw & forward \\
\hline G & Guanosine \\
\hline g & Gram / centrifugal force \\
\hline GTP & Guanosine triphosphate \\
\hline $\mathrm{h}$ & Hour \\
\hline h. sapiens & Homo sapiens \\
\hline $\mathrm{H}_{2} \mathrm{O}$ & Water \\
\hline $\mathrm{HCl}$ & Hydrochloric acid \\
\hline HeLa & Henrietta Lacks \\
\hline HEPES & 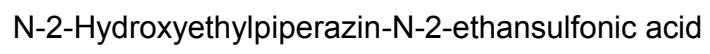 \\
\hline $\mathrm{hn}$ & Heterogenous nuclear \\
\hline IBC & Intron binding complex \\
\hline ILS & Intron lariat spliceosome \\
\hline IPTG & Isopropyl- $\beta$-D-thiogalactopyranosid \\
\hline ISE & Intronic splicing enhancer \\
\hline ISL & Internal-stem loop \\
\hline ISS & Intronic splicing silencer \\
\hline $\mathrm{J}$ & Joule \\
\hline k & Kilo \\
\hline $\mathrm{kb}$ & Kilo bases \\
\hline $\mathrm{KCL}$ & Potassium chloride \\
\hline
\end{tabular}




\section{Appendix}

$\mathrm{kDa}$

L

LB

LC

Lsm

$\mathrm{m}_{3} \mathrm{G}$

$\mathrm{m}^{7} \mathrm{G}$

M

m

$\mu$

MBP

$\min$

$\mathrm{MgCl}_{2}$

$\mathrm{mM}$

mRNA

MS

MW

$\mathrm{N}$

$\mathrm{n}$

NTD

NTP

nt

OD

$-\mathrm{OH}$

PI

PI

$\mathrm{P}$

$\mathrm{p}$

PAGE

$\mathrm{PCl}$

PCR

$\mathrm{pH}$

PMSF

PY

Pre-mRNA

$\%$

$\mathrm{R}$

RES
Kilodalton

Liter

Luria Bertani

Liquid chromatography

Like-Sm

2,2,7-trimethylguanosine

N7-methylguanosine

Molar

Milli / meter

Micro

Maltose-binding protein

Minutes

Magnesium chloride

Millimolar

Messenger RNA

Mass spectrometry

Molecular weight

Amino

Nano

$\mathrm{N}$-terminal domain

Nucleoside triphosphate

Nucleotides

Optical density

Hydroxyl group

Pre-mRNA I

Pre-mRNA II

Phosphate

Pico

Polyacrylamide gel-electrophoresis

Phenol-chloroform-isoamyl alcohol

Polymerase chain reaction

Preponderance of hydrogen ions

Phenylmethylsulfonylfluoride

Polypyrimidine

Precursor-mRNA

Percent

Purine base

Retention and splicing 


\begin{tabular}{|c|c|}
\hline Rev & reverse \\
\hline RGG & Arginine glycine glycine repeats \\
\hline RNA & Ribonucleic acid \\
\hline RNAi & RNA interference \\
\hline RNase & Ribonuclease \\
\hline RNP & Ribonucleoprotein \\
\hline rpm & Revolutions per minute \\
\hline RRM & RNA recognition motif \\
\hline RT & Room temperature/ Reverse transcription \\
\hline S & Svedberg unit \\
\hline s & Second \\
\hline S. cerevisiae & Saccharomyces cerevisiae \\
\hline S. pombe & Saccharomyces pombe \\
\hline SDS & Sodium dodecylsulfate \\
\hline SE & Splicing extract \\
\hline siRNA & Small interfering RNA \\
\hline Sm & 'Smith', patient in which Sm proteins were first discovered \\
\hline sn & Small nuclear \\
\hline snRNA & Small nuclear ribonucleic acid \\
\hline snRNP & Small nuclear ribonucleoparticles \\
\hline SR & Serine arginine rich \\
\hline $\mathrm{T}$ & Thymin \\
\hline TEMED & $\mathrm{N}, \mathrm{N}, \mathrm{N}$ ', N'-Tetramethylethylendiamine \\
\hline Tris & Tris-(hydroxymethyl)-aminomethane \\
\hline tRNA & Transfer RNA \\
\hline$U$ & Uridine / unit \\
\hline U snRNA & Uridine rich small nuclear RNA \\
\hline U snRNP & Uridine rich small nuclear ribonucleoparticles \\
\hline UTP & Uridine triphosphate \\
\hline UV & Ultraviolet \\
\hline V & Volt \\
\hline Vol & Volume \\
\hline$v / v$ & Volume per volume \\
\hline W & Watts \\
\hline$w / v$ & Weight per volume \\
\hline y & yeast \\
\hline Y & Pyrimidine base \\
\hline
\end{tabular}




\section{Appendix}

\subsection{List of figures}

Figure 1.1 Conserved splicing sequences of pre-mRNA from $H$. sapiens and $S$. cerevisiae......................

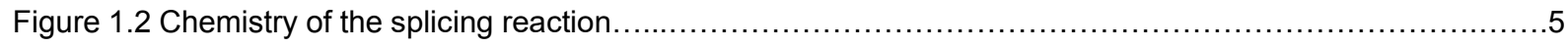

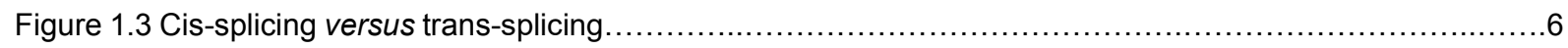

Figure 1.4 Sequences and conserved secondary structures of the human spliceosomal snRNAs.....................8

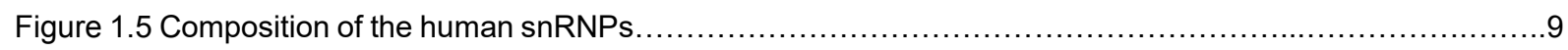

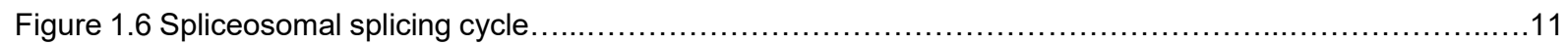

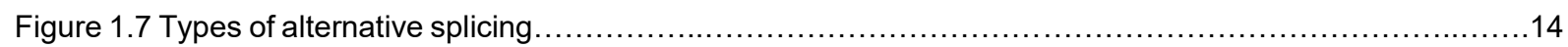

Figure 1.8 Molecular interactions during spliceosome assembly across an exon..............................16

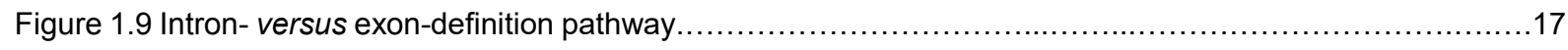

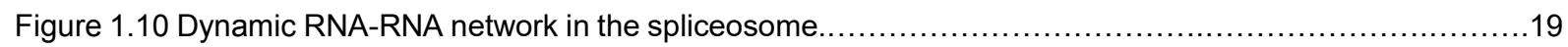

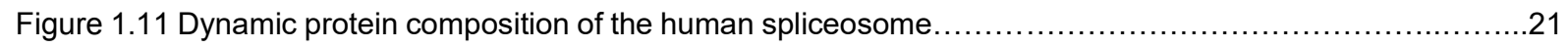

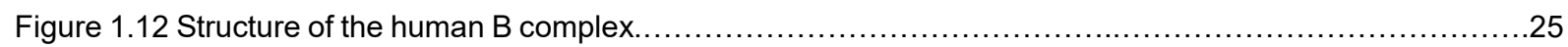

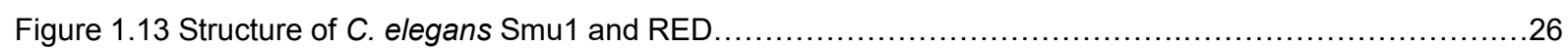

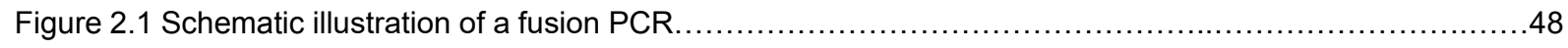

Figure 3.1 Splicing events affected by siRNA-mediated depletion of Smu1, RED or MFAP1 ....................62

Figure 3.2 siRNA-mediated depletion of Smu1 or RED results in retention of short constitutively spliced

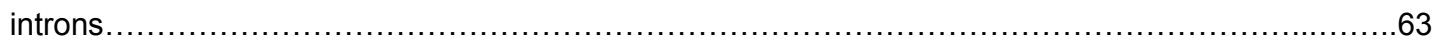

Figure 3.3 Smu1 and RED levels are efficiently and specifically decreased by immunodepletion.................65

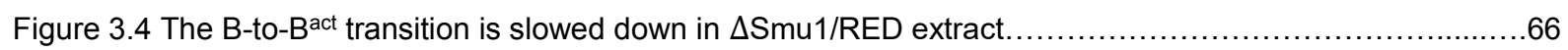

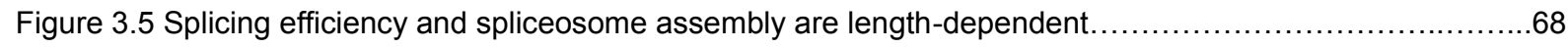

Figure 3.6 Splicing and spliceosome assembly are slowed down/inhibited by the Smu1/RED depletion...........69

Figure 3.7 Purified recombinant Smu1/RED is sufficient to restore spliceosome activation and splicing............71

Figure 3.8 Depletion of Smu1/RED stalls spliceosome assembly at the B complex level.........................72

Figure 3.9 PY tract extension does not compensate for a short 5 'SS-BS distance............................78

Figure 3.10 MINX-80-cleaved allows spliceosome activation and splicing in the absence of Smu1 and RED.....80

Figure $3.11 \Delta$ Smu1/RED B complexes can be chased into activated spliceosomes............................82

Figure 3.12 The interaction between Smu1 and RED appears to be important for their function...................84

Figure 3.13 The interaction of Smu1 and RED enhances their association with the spliceosome...................85

Figure 3.14 The WD40 domain of Smu1 is important for interaction of the dimer with the spliceosome and for its function.

Figure 3.15 RED contributes to the function of the Smu1/RED dimer. .87

Figure 3.16 Spliceosomes formed on MINX-70 contain higher amounts of U1............. .88 
Appendix

Figure 3.17 Phosphorylation of Prp31 and SF3b155

Figure 3.18 siRNA-mediated depletion of Prp38, MFAP1, Smu1 and RED results in altered phosphorylation of Prp31 and SF3b155. .95

Figure 3.19 Procedure used for the purification of endogenous spliceosomes...... .97

Figure 3.20 RNA composition of endogenous spliceosomes precipitated with anti-P-Prp31 antibodies. .98

Figure 4.1 Location of Smu1 and RED within the human B complex. 102

Figure 4.2 Location and interactions of the pre-mRNA within the human B complex...... 108

Figure 4.3 Model for how the 5'SS-BS distance affects the dependency of spliceosome activation on 


\section{Appendix}

\subsection{List of tables}

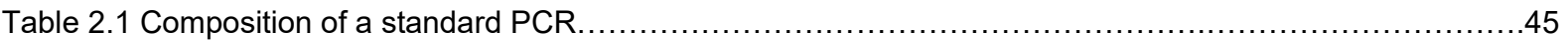

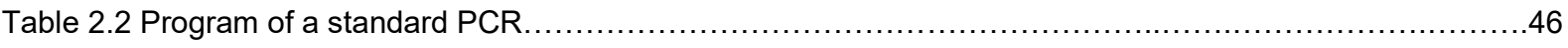

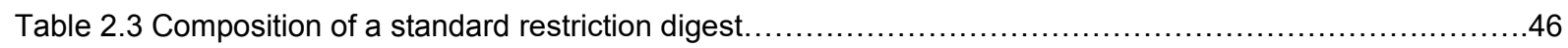

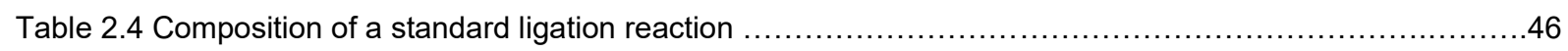

Table 2.5 Composition of reaction mixtures for the first and third amplifications................................. 49

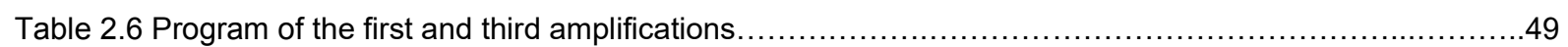

Table 2.7 Composition of reaction mixtures for the second amplification (annealing).............................49

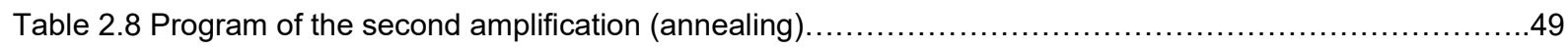

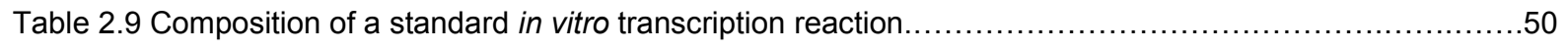

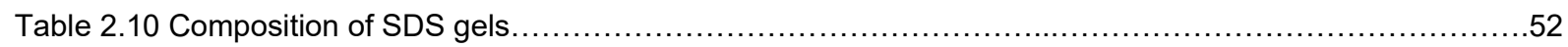

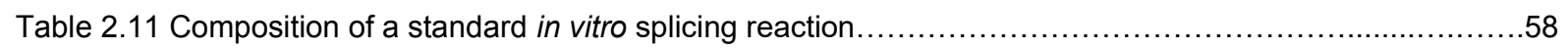

Table 3.1 Protein compositions of the $\Delta$ Smu1/RED and mock (kinetically stalled) B complexes..................73

Table 3.2 Protein compositions of spliceosomal complexes assembled on MINX-70, -80 and $-120 \ldots \ldots \ldots \ldots \ldots . . .89$ 


\section{Appendix}

\subsection{Acknowledgements}

After 10 years of studying I would like to wholeheartedly thank everyone who accompanied, supported and motivated me during this exceptional time.

Firstly, I want to express my sincere gratitude to my advisor and mentor Prof. Dr. Reinhard Lührmann for the opportunity to work on this challenging and enthralling project and for the continuous support, motivation and intellectual input during the entire time of research and writing.

A very special thanks to Dr. Cyrille Girard. Thank you for five years of great supervision, of working side-by-side in the lab and of enjoying delightful lunches. I appreciate that you sheared your fascinating and challenging project with me and all the things I learned from you. It was an exciting time.

Furthermore, I would like to thank the rest of my Thesis Committee - Prof. Dr. Heike Krebber and Prof. Dr. Patrick Cramer for listening and for providing another perspective on my project every once in a while. I further want to express my thanks to Prof. Dr. Markus Zweckstetter, Prof. Dr Jörg Großhans and Prof. Dr. Ralph Kehlenbach, who immediately have expressed their willingness to become members of my extended Thesis Committee.

I would also like to express my greatest appreciation to Evelina De Laurentiis for work-related help, but mainly for becoming a good friend of mine and for sharing so many precious moments with me. I also want to thank Leyla El Ayoubi and Vinay Kumar, not only for scientific support but also for having essential chocolate and cookie time with me.

I further want to acknowledge my dear lab companions Reinhard Rauhut, Ulrich Steuerwald, Majety Naga Leelaram and Winni Lendeckel for nice conversations, the sound of music and being considerate during my time of writing.

Additionally, I want to acknowledge Cindy Will for encouraging me and for helping me with her brilliant mind, Monika Raabe and Annika Reinelt for conducting MS analysis for me, llya Komarov for his effort to resolve the structures of my complexes by electron microscopy and Juan Valcárcel and Panagiotis Papasaikas for their cooperation for the RNAseq analysis and Berthold Kastner for providing numerous structural illustrations.

I also want to thank Gabi, Irene, Gertrud, Claudia, Kami, Juliane und Marion for making life in lab a bit easier for all of us every day. 


\section{Appendix}

With all my heart I want to thank my plethora of precious friends who not only dragged me through the tough phases of my academic career and private life, but also shared so many unforgettable moments with me. Thanks to Annpu, Tine, Philipp, Kamila, Maria und Isa for an amazing time in high school. Thanks to Kiki, Kathy, Ines and Wencke! I owe my B.Sc. and my sanity to you. Thanks to Berti, Ali and Inga for being who you are! I hope I will never be without the privilege of having you in my life! Thanks to Anna for travelling to New Zealand with me to visit Berti. It was the most affecting and exciting trip in my life and it was perfect to share this experience with you. Thanks to Chrissy, Ingi, Cindy, Bine and Lisi and for still being in my life! Thanks to Katha for understanding and for so many nice moments together. Thanks to Jojo!?! For seeing the world as you do. Not to forget Jana and Flora! For always being there for me and for being as amazing as you are! Inka would be proud of you!

All my love and gratitude I want to express to Linus for supporting me in every way possible. You make every day worth living.

Last but not least I want to thank my Family; my parents Maria and Roland and my sister Sabrina for always believing in me more than I do and for supporting me financially, emotionally and also actively. Thanks to my grandparents Adelaide and Eduardo and to Graça, Derek, Darrel and Delisa as well as my recently gained extended family. I am fortunate to have you in my life! 